\title{
COMPARING ANTI-VEGF ANTIBODIES AND APTAMERS ON PAPER MICROFLUIDIC-BASED PLATFORMS
}

\author{
A Thesis \\ presented to \\ the Faculty of California Polytechnic State University, \\ San Luis Obispo
}

\author{
In Partial Fulfillment \\ of the Requirements for the Degree \\ Master of Science in Biomedical Engineering
}

by

Katherine Noel Clayton

June 2012 
(C) 2012

Katherine Noel Clayton

ALL RIGHTS RESERVED 


\section{COMMITTEE MEMBERSHIP}

TITLE:

AUTHOR:

DATE SUBMITTED:

COMMITTEE CHAIR:

COMMITTEE MEMBER:

COMMITTEE MEMBER:
Comparing Anti-VEGF Antibodies and Aptamers on

Paper Microfluidic-Based Platforms

Katherine Noel Clayton

June 2012

Dr. David Clague, Associate Professor

Dr. Lily Laiho, Associate Professor

Dr. Daniel Walsh, Associate Professor 


\begin{abstract}
COMPARING ANTI-VEGF ANTIBODIES AND APTAMERS ON PAPER
\end{abstract}

MICROFLUIDIC-BASED PLATFORMS

By

Katherine Clayton

The field of microfluidics is expanding into what is known as paper microfluidics. This uses a paper platform rather than materials (i.e. PDMS, PMMA) that are commonly used in microfluidics research. Current devices require an expensive manufacturing process and external sources to power the device. Such devices are not practical in low resource environments. As a consequence, it is the goal of this Thesis to develop a threedimensional, multiplexed assay chip using nitrocellulose membranes. This device comprises of multiple layers of nitrocellulose membranes with defined fluidic channels. The multiple layers are bound together using double backed tape, and imbedded between the layers are conjugate reagents. In the detection region both antibodies and aptamers were evaluated.

The fiberglass pad where conjugate reagents would be contained, were initially saturated in dye. As sample was inputted into the three-dimensional chip, the fluid path could be visualized. Without the use of the conjugate pad the chip's four detection regions showed detection within one minute of one another. However, the addition of this fibrous pad skewed time points dramatically. The hypothesis that a three-dimensional chip could be designed to detect different biomarkers in a multi-analyte sample was satisfied. However, simultaneous detection was only possible if the conjugate pad was either neglected or, possibly, a different material was used.

Additionally, current lateral flow assay technologies, another research area that paper microfluidics spawns from, use antibodies in order to capture biomarkers in sample and provide visual signal to the user. However, antibodies are sensitive to denaturation with $\mathrm{pH}$ and temperature, whereas aptamers can withstand much more extreme environmental conditions. A two-dimensional nitrocellulose chip was designed to compare antibodies and aptamers as capture reagents to detect VEGF, using colloidal gold as a particle to visualize detection. Both monoclonal and polyclonal anti-VEGF antibodies were used and showed no signal. On the other hand, the anti-VEGF aptamer produced a visual signal when conjugated to biotin on its 5' end. This data was further validated by a separate project analyzing the binding kinetics of the antibody and the aptamer using Surface Plasmon Resonance. Therefore, the hypothesis that aptamers could be used as a possible capture reagent in a paper microfluidic chip for the detection of VEGF was satisfied.

Keywords: paper microfluidics, three-dimensional, VEGF, antibody, aptamer 


\section{Acknowledgements}

I would like to thank the faculty and staff in the Biomedical and General Engineering department for their work and support throughout my undergraduate and master's education. I appreciate the time, knowledge, and encouragement that Dr. David Clague has displayed throughout the duration of this thesis project. His advice was vital in proceeding through such a project. I would also like to extend an additional thank you to Dr. Daniel Walsh and Dr. Lily Laiho in providing their time and help as committee members on this thesis defense. Furthermore, I would like to show my gratitude toward the Office of Naval Research, for which without their funding this project would not have been possible.

To my friends and colleagues I have come across throughout my years at this university, thank you for the challenges provided both in and outside of the classroom, aiding in the true, "learn by doing" mantra that comes with being a true Poly student. Last, but definitely not least, I would like to thank my parents Gary Clayton and Margaret Clayton. Their never-ending love and support aided in my success as a student at Cal Poly and my future beyond the limits of this institution. 


\section{Table of Contents}

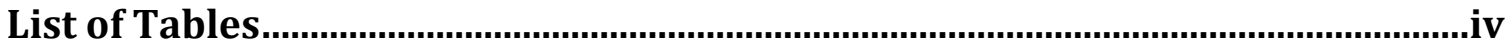

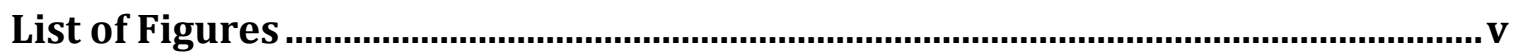

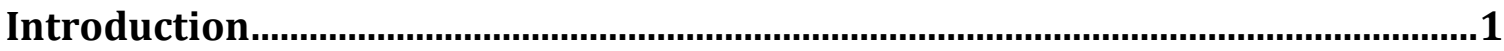

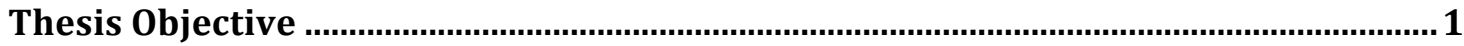

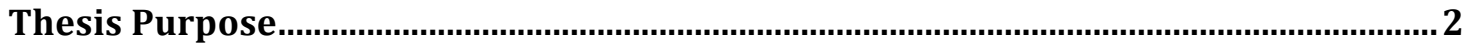

Diagnostics for Global Health ......................................................................................

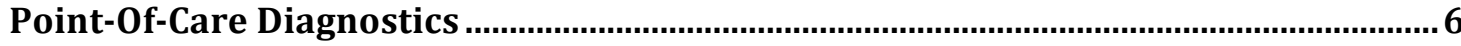

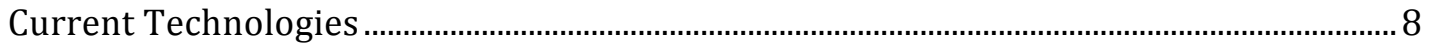

Lateral Flow Assays and Immunochromatography................................................... 10

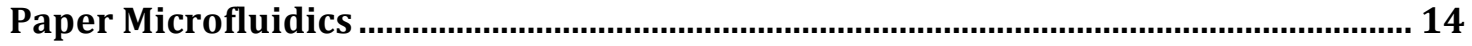

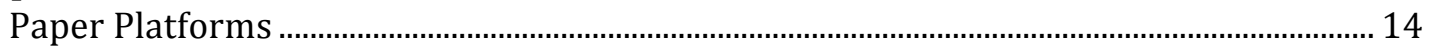

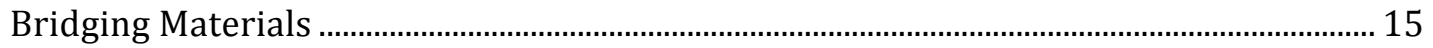

Capture and Detector Reagents ....................................................................................................15

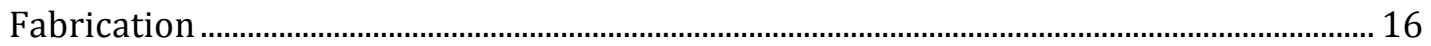

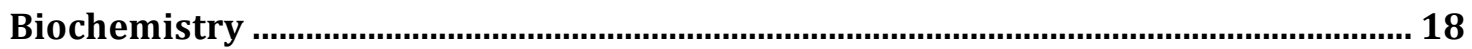

Vascular Endothelial Growth Factor............................................................................................ 19

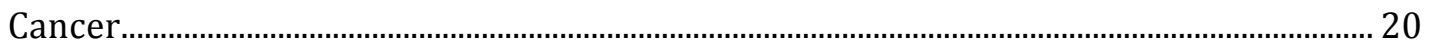

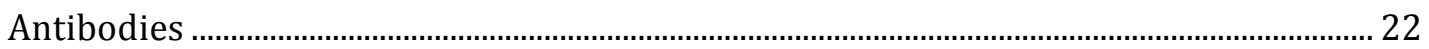

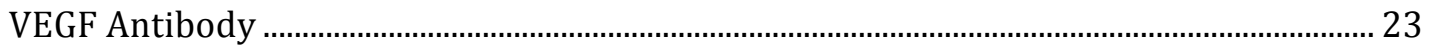

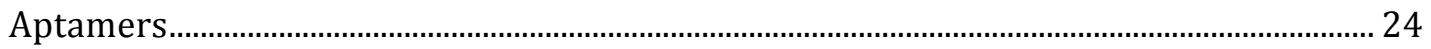

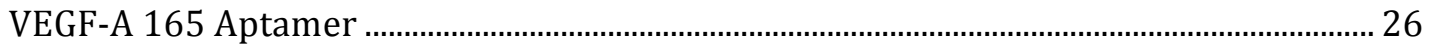

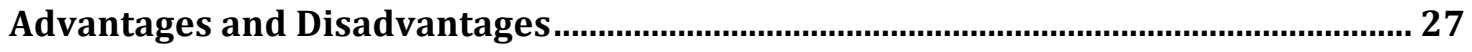

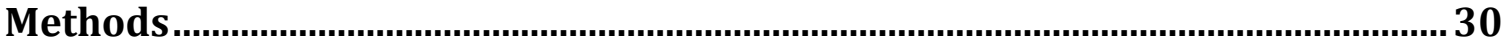

Characterization

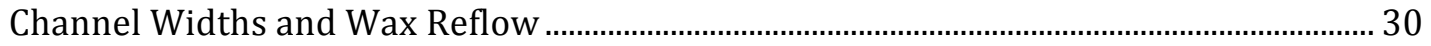

Optimal Channel Characteristics ..................................................................................................... 33

Design Combinations and Wicking Times....................................................................................... 33

Wicking Time - Conjugate Pad vs. Membrane Paper ………………………………………. 37

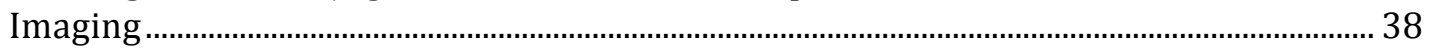

Development of a Three-Dimensional Paper Microfluidic Chip .................................. 38

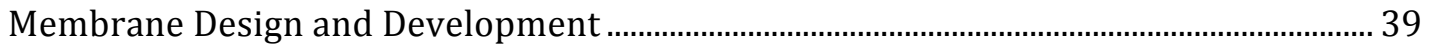

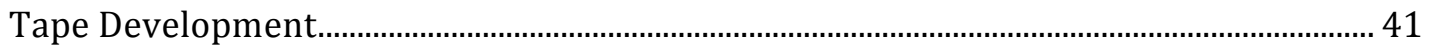

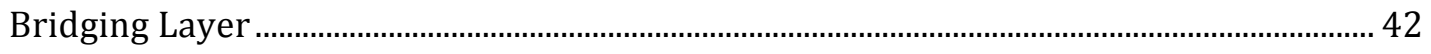

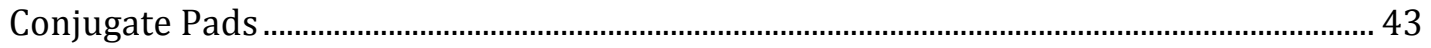

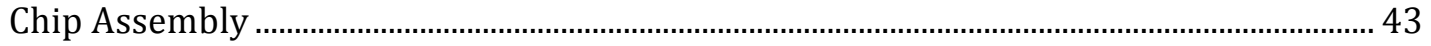

Testing of Three-Dimensional Chip ....................................................................................... 44

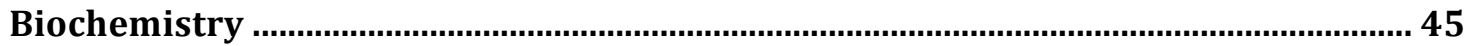

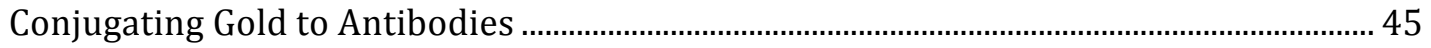

Monoclonal Antibody Detection …………………………………………………………….... 48

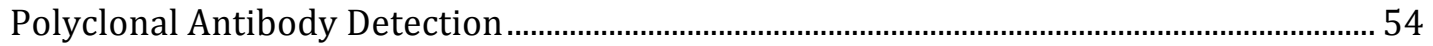

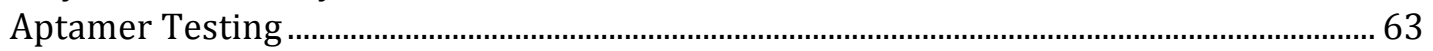

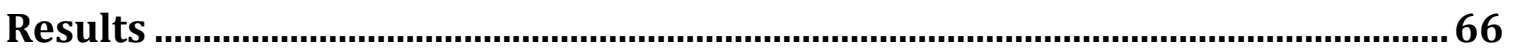

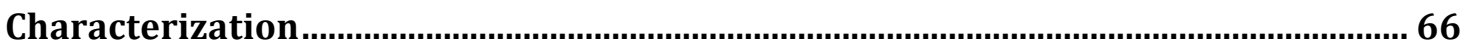

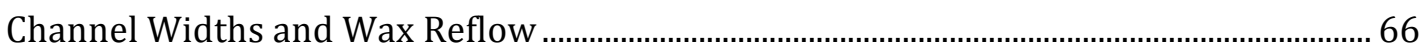

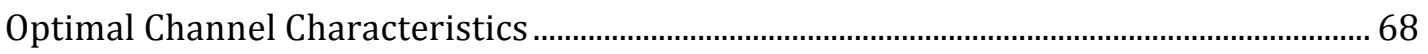

Design Combinations and Wicking Times...............................................................................6 
Wicking Time - Conjugate Pad vs. Membrane Paper ……....................................................... 71

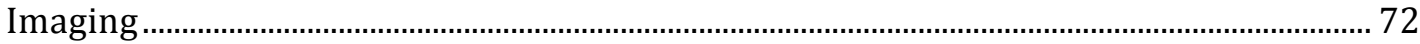

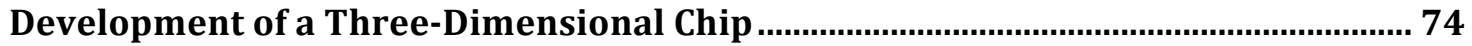

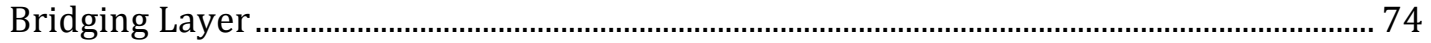

Testing of Three-Dimensional Chip ................................................................................... 76

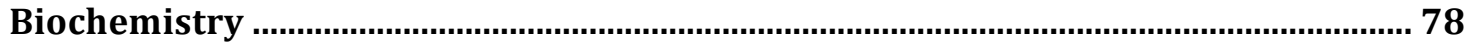

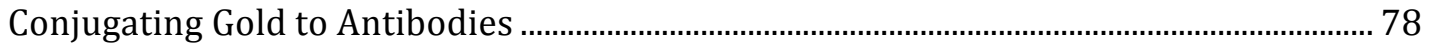

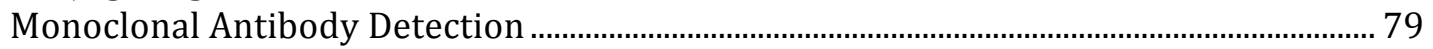

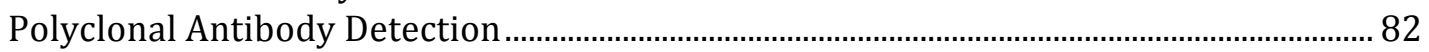

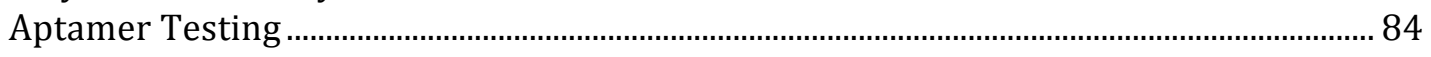

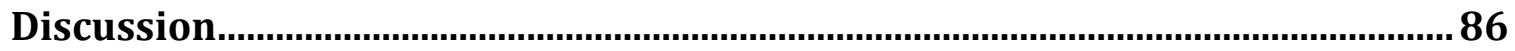

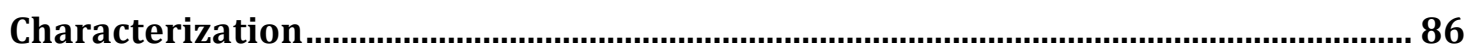

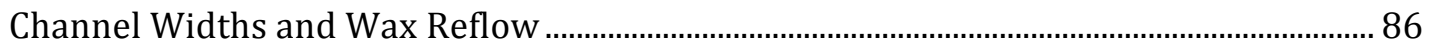

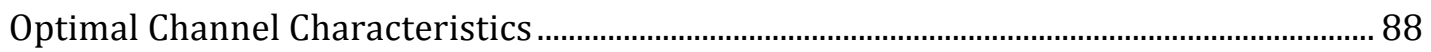

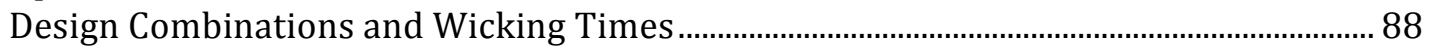

Wicking Time - Conjugate Pad vs. Membrane Paper ................................................................. 93

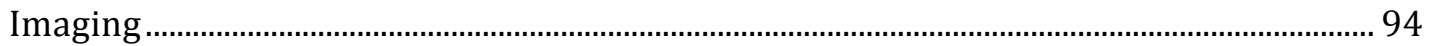

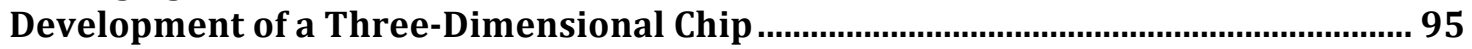

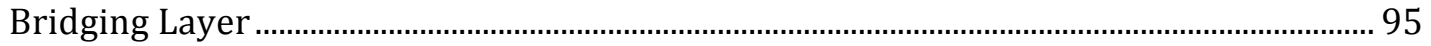

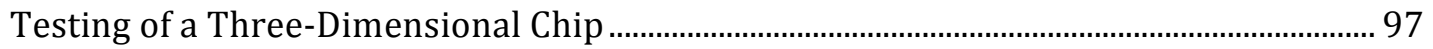

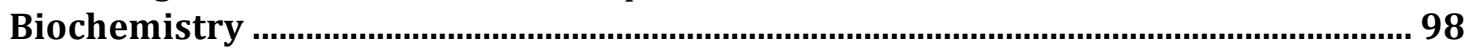

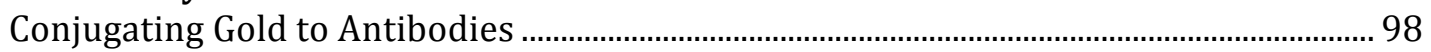

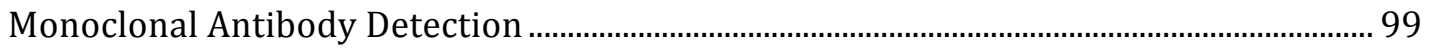

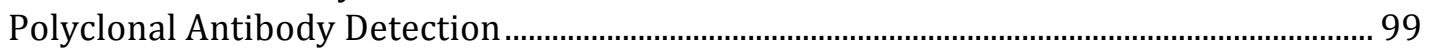

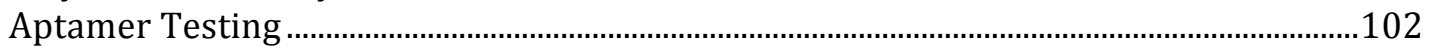

Conclusion .................................................................................................................. 104

Future Work

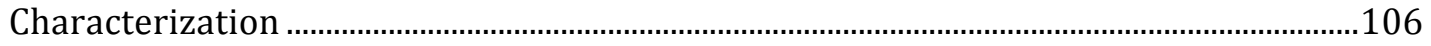

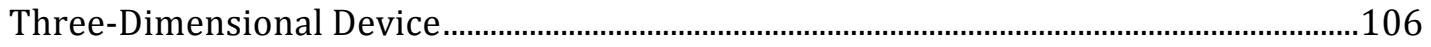

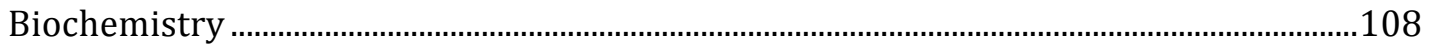

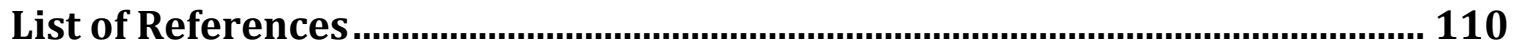

Appendix A: Statistical Analyses for Chip Design Characteristics.................... 114

Appendix B: Three-Dimensional AutoCAD Chip Drawings and Designs ......... 147

Appendix C: Two-Dimensional AutoCAD Chip Drawings and Designs............. 173

Appendix D: Biochemistry Dilution Calculations.................................................. 185

Appendix E: Instructions on Running A BLAST Test........................................... 193

Appendix F: Biochemistry Test Combinations................................................... 196

Appendix G: Biochemistry Optimal Concentration Calculations....................... 202

Appendix H: SEM Images of Nitrocellulose........................................................... 214 


\section{List of Tables}

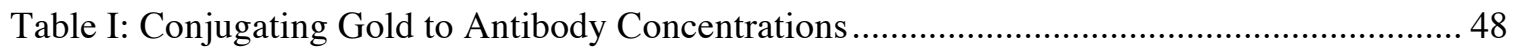

Table II: Post-treatment and Washes for Monoclonal anti-VEGF Antibody ............................... 53

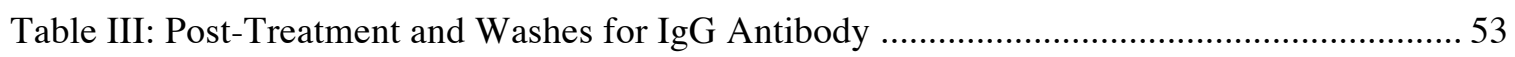

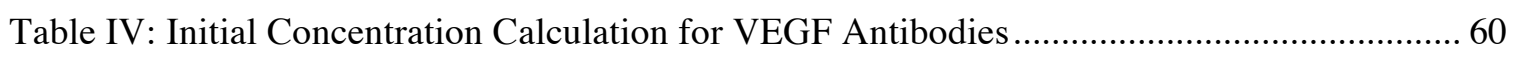

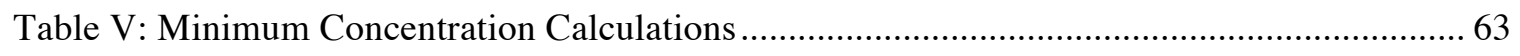

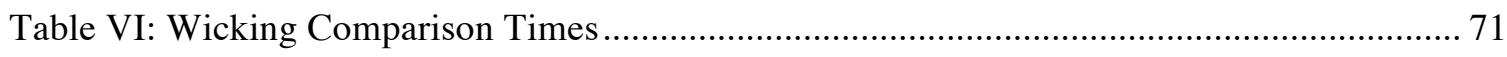

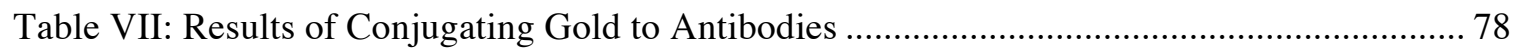

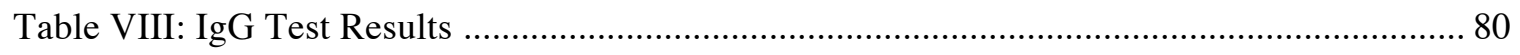

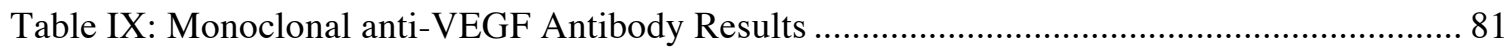

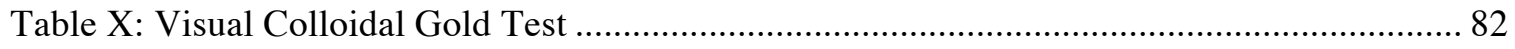

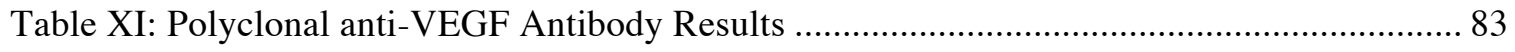

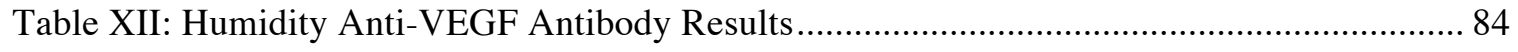

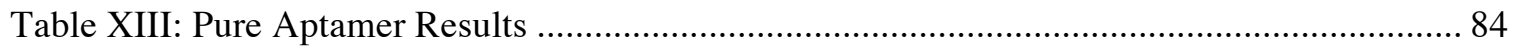

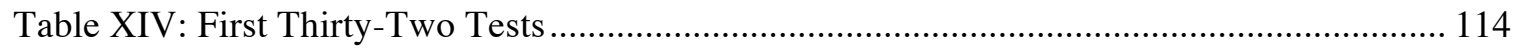

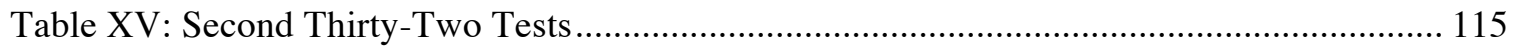

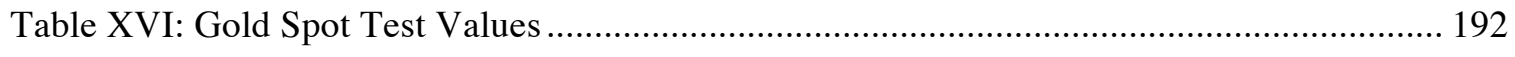

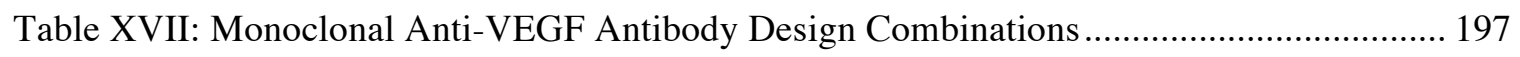

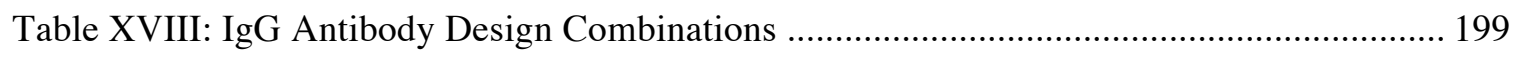

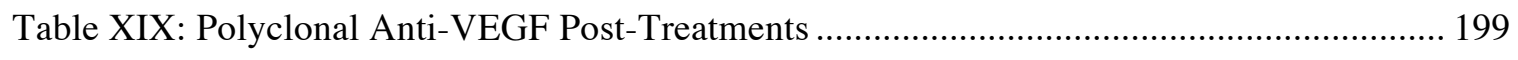

Table XX: Polyclonal Anti-VEGF Antibody Design Combinations .......................................... 200

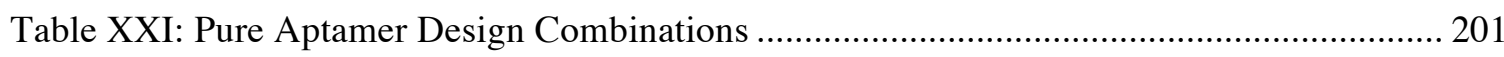




\section{List of Figures}

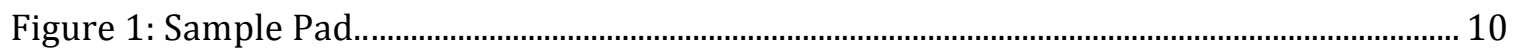

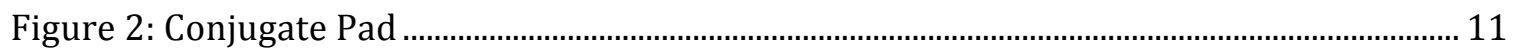

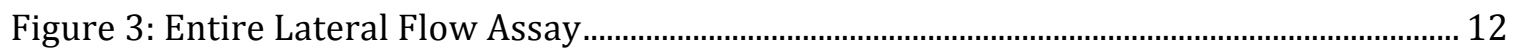

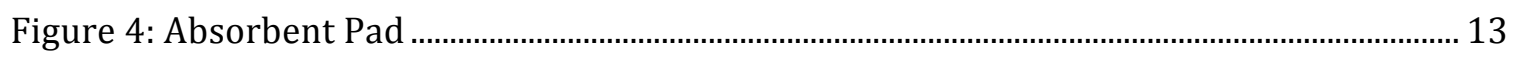

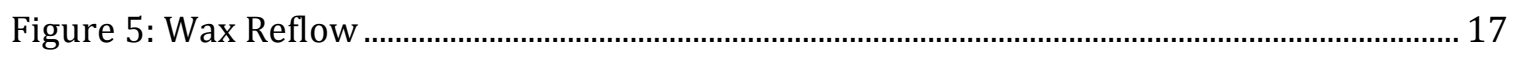

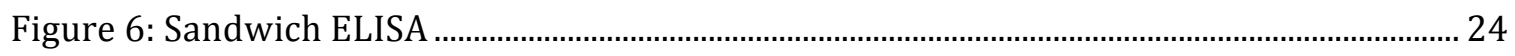

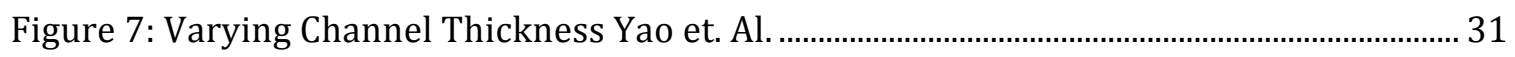

Figure 8: Dimensioned Drawing for Channel Thickness ………………...................................... 32

Figure 9: Wax representation of Channel Thickness ................................................................................ 32

Figure 10: Conjugate Pad vs. No Pad ............................................................................................ 38

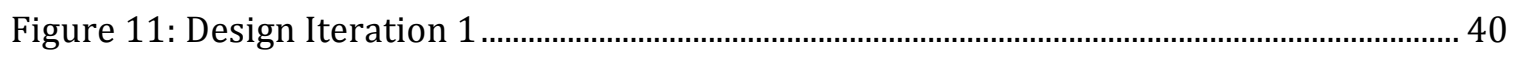

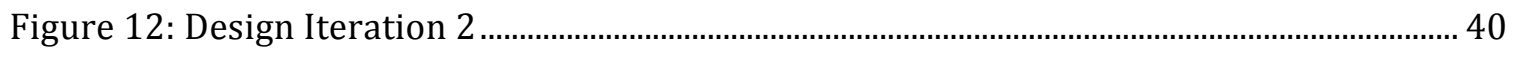

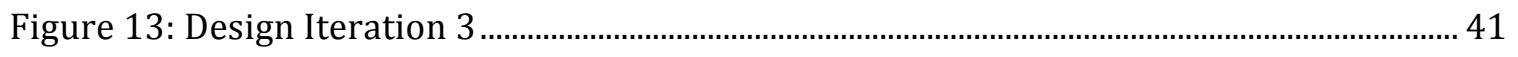

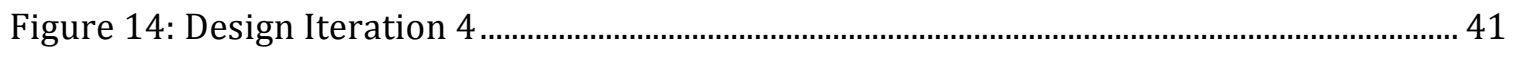

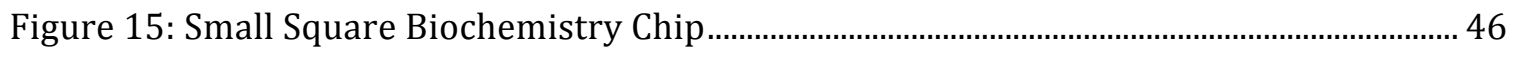

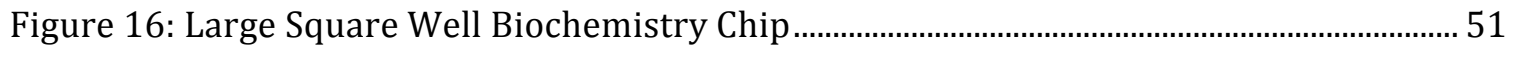

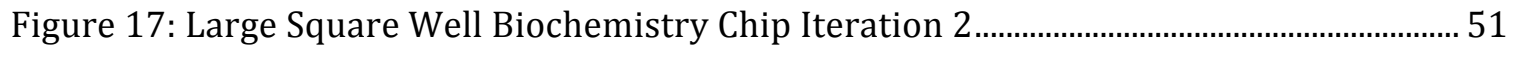

Figure 18: Jumper Design Biochemistry Chip ................................................................................... 52

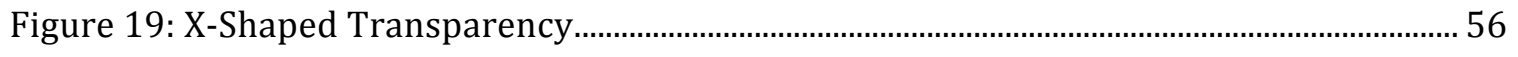

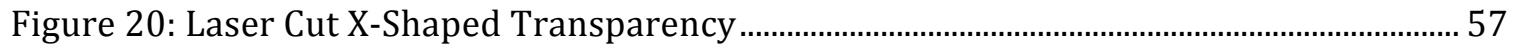

Figure 21: Labeled Circular 2D Chips for Biochemistry ………................................................ 57

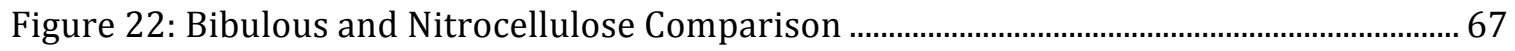

Figure 23: Channel Thickness Wicking Comparison ........................................................................... 68

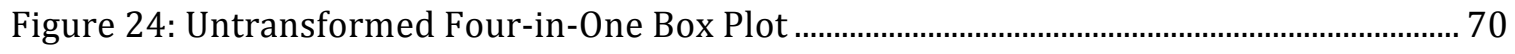

Figure 25: Transformed Four-in-One Box Plot ................................................................................. 71 
Figure 26: Smallest Nitrocellulose Pore Size SEM Image ……….................................................... 72

Figure 27: Wax Impregnated Membrane on SEM ............................................................................. 73

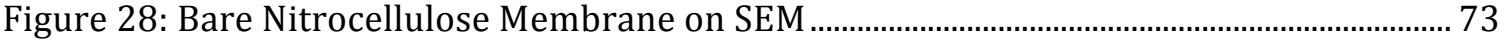

Figure 29: Seam Between Wax and Non-Wax on SEM ……............................................................. 74

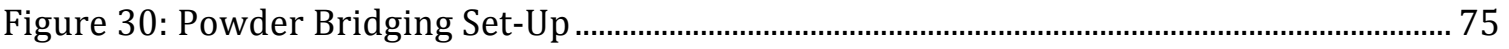

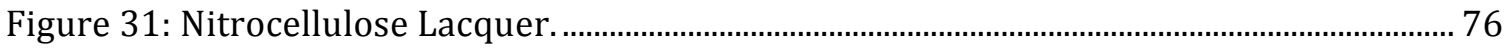

Figure 32: Application of Nitrocellulose Lacquer ............................................................................... 76

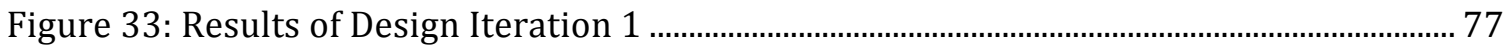

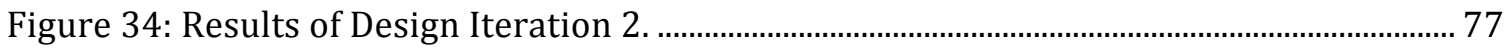

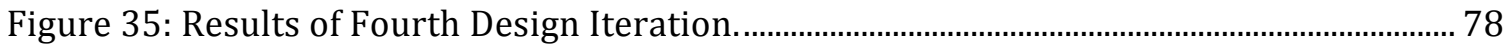

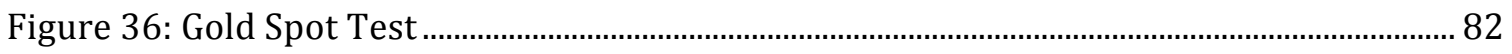

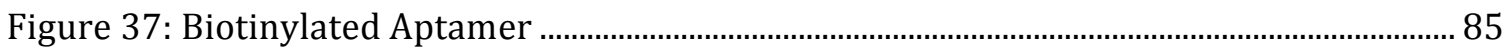

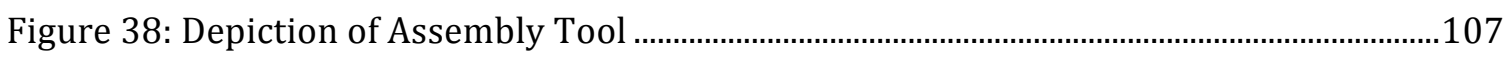

Figure 39: Conceptual design of diagnostic chip reader..............................................................109

Figure 40: 2D Two Analyte Detection Design................................................................................172

Figure 41: 2D Three Analyte Detection Design ...............................................................................172

Figure 42: 3D Six Analyte Detection Design.................................................................................172

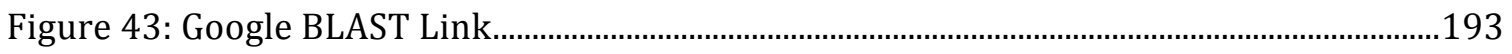

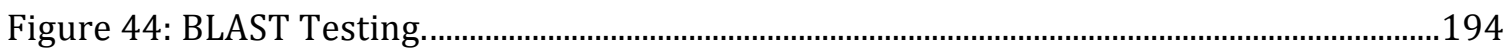

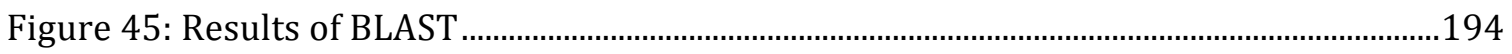

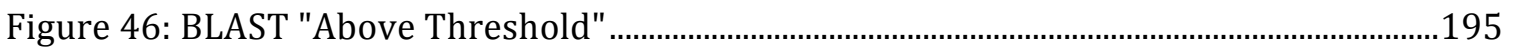

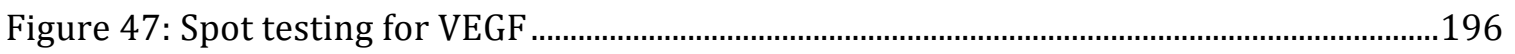

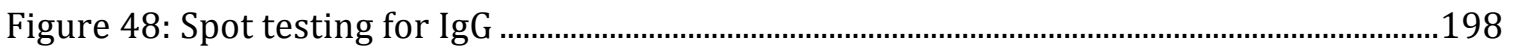

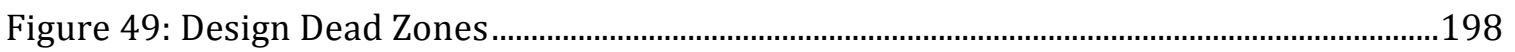

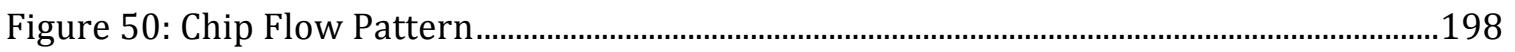

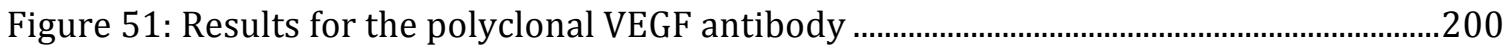




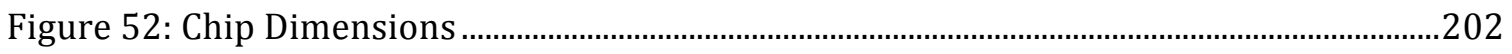

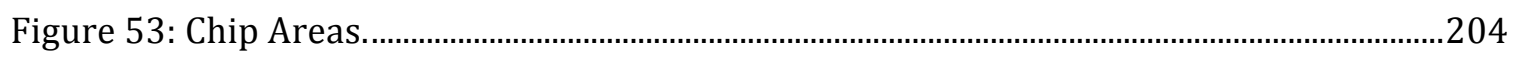

Figure 54: Described X-Shaped Design ..........................................................................................205

Figure 55: HF 135 SEM 2500X Objective.......................................................................................214

Figure 56: Wax HF 135 SEM 1000X Objective ...........................................................................214

Figure 57: Bare HF 135 SEM 1000X Objective.............................................................................215 


\section{Introduction}

Paper microfluidics encompasses concepts used in traditional microfluidics in conjunction with lateral flow assays [1]. This research area allows for both sensitive and accurate detection of particles in a sample while maintaining low cost for each device. This technology - commonly referred to as a lab-on-a-chip (LOC) - is being further developed within the biomedical engineering field [2]. Advancement of this technique would allow for an alternative for disease diagnosis for both those in the developed and developing world. Paper microfluidics is an advantageous approach to diagnostics because it produces clear results and requires minimal training from the user [3]. By exploring this field further, it would allow for the dramatic innovation and improvement in diagnostics.

\section{Thesis Objective}

There are three main objectives of this thesis.

- Characterize properties of nitrocellulose as a material platform for custom lateral flow assays.

- Design, manufacture, and test a simultaneously detecting threedimensional multi-layer chip as a potential inexpensive and simplistic solution for multiplex point-of-care diagnostics.

- Understand and implement biochemistry into the device by comparing antibodies and aptamers as potential solutions for detecting the growth factor, VEGF (vascular endothelial growth factor) [4]. 


\section{Thesis Purpose}

The purpose of this thesis is to determine the feasibility of developing a threedimensional paper microfluidic device in-house that can simultaneously detect an inputted sample in multiple detection regions of the chip. Additionally, this thesis will compare the ability of using antibodies compared to aptamers as potential capture reagents to produce a visual signal if a particular concentration of VEGF is detected by the sample.

\section{Diagnostics for Global Health}

For a country to be considered as developed or developing it is either measured by its gross domestic product (GDP) per capita or Human Development Index (HDI) $[5,6]$. The GDP measurement is purely economical, where a high income per capita would describe a developed country [5]. However, an HDI takes into account economy, income, life expectancy, and education. The higher ratings also denote a developed country [6]. Although these exist as possible quantitative measurements to explain a country's development, generally, a developed country is defined as, “...one that allows all its citizens to enjoy a free and healthy life in a save environment" according to Kofi Annan (the former Secretary General of the United Nations) [7]. These definitions and concepts become important for understanding the dramatic differences in healthcare that is provided in these two different categories.

In a developed country, disease diagnostics development is important because it provides more timely care to its patients, it helps to ensure safe blood banking, and it provides surveillance data for future emergency public health situations and long-term public health strategies [3]. Focusing on timely care to patients, in the developed world, a patient commonly goes to their general practitioner to seek initial medical advice. When 
their blood, urine, or stool sample is taken, it is commonly sent to a laboratory either within the hospital or externally and results come back at a later time [3]. However, it would be beneficial to eliminate this wait time. Paper microfluidics allows for a doctor or nurse to evaluate patient health locally within minutes.

In the developing world the need for paper microfluidics is just as important, if not more, than that in developing countries [3]. Paper microfluidics are a form of pointof-care (POC) device. This means that a patient can be diagnosed on site, or at the point, in which they are receiving medical care. This could be at a hospital, local clinic, or even at home [3]. These POC devices are favorable in developing countries because they provide simple, reasonably accurate results that require minimal training, which is beneficial in low resource situations. One issue that must be addressed, however, is that most organizations that focus on healthcare in the developing world invest more heavily in drug discovery and vaccines rather than diagnostics, so that designing and implementing an low-cost device becomes even more crucial [3, 8].

Currently, there are no standards that are followed for diagnosing patients in the developing world [3]. Because of this, misdiagnosis is common [9]. However, the World Health Organization (W.H.O.) is working to implement a set of guidelines that they have developed, which provides a list of clinical symptoms and the appropriate examinations for them. The W.H.O. created this protocol in order to minimize misdiagnosis [3]. A serious issue in the developing world is that unnecessary medication is given to misdiagnosed patients. Although this may initially seem harmless, these inessential pharmaceuticals have led to haphazard spending, and eventual resistance of the disease to the drugs. Although the guidelines provided by the W.H.O. have led to a reduction in 
disease burden worldwide, there is still significant room for improvement. The ambiguous directions continue to lead to false diagnosis and incorrect treatment of the patient [3].

When approaching the development of diagnostic devices, the concept of appropriate technology (AT) must be considered. Appropriate technology is defined as the design and development of any object that can be appropriately used in the community that it is provided to. The World Health Organization specifies that the development of AT devices should follow the acronym "ASSURED” (Affordable, Sensitive, Specific, User-friendly, Rapid and Robust, Equipment-free, Deliverable) [10]. The importance of an affordable device is that people of all socio-economic backgrounds will be able to afford diagnostic healthcare [10]. In the developing world, a large portion of the population is of low income, and a small fraction has higher income, with a nonexistent middle-class. Commonly, these countries have private hospitals that only the affluent population can afford [3]. The rest of the population receives medical care from a low-resource clinic, or none at all. In light of these facts, it is crucial that low cost devices be developed that are sensitive enough to ensure rapid, first-time detection [10]. The sensitivity of the device saves repeat testing which lowers cost for the facility and patient. Specificity of the device, like sensitivity, must allow for accurate results, rather than inconclusive ones [10]. Since the medical equipment could be handled by anyone, from a doctor to a local volunteer, the diagnostic needs to be user-friendly so that anyone can determine its results [10]. At the moment, microscopes are the most common diagnostic tool in developing nations [3]. Although a powerful and sensitive device, it is not userfriendly. The lack of the appropriate instrumentation and training commonly leads to 
erroneous results from the untrained eye. A rapid and robust test is advantageous because a quick turnaround time would help aid in immediate treatments for the patients. Also, the device could be used in a hospital, a clinic without power, or on the battlefield [3]. An equipment-free device frees diagnostics from a power source, which tends to be unreliable in many parts of the world [3]. Additionally, less equipment would aid in affordability of the device. Finally, a deliverable device would be easily transportable to the area in which the diagnostic is needed at a minimal cost while maintaining its integrity [10].

There is a clear need to for accurate and simple disease diagnosis in low-income countries. In the developing world, malaria, HIV, and tuberculosis comprise of $95 \%$ of the disease burden [3]. These diseases are commonly referred to as, "the big three". These deleterious diseases often affect adults in developing nations, which leads to a considerable number of deaths. As a result, numerous children are orphaned throughout areas where these diseases run rampant and healthcare is not readily available. With that said, many children in the developing world are commonly affected by diarrheal diseases and acute respiratory infections [3]. The places in which these infectious diseases impinge the population compliment the fact that there is a lack of infrastructure in the area [3]. As these nations develop their infrastructure, the disease burden shifts from the plague of infectious diseases to non-communicable ones [1]. Additionally, with this shift there is an increase in obesity and diabetes. Looking at high-income countries, the noncommunicable diseases that plague a large percentage of the population are ischemic heart disease and cerebrovascular disease [3]. However, it is important to note that the developing world is facing a larger population affected by cancer - once thought to be a 
disease that only affected the developed world $[11,12]$. Therefore, there are numerous of diseases that can be further addressed through the advancement of diagnostic devices.

Further focusing on diagnostics of infectious disease in the developing world, there are many issues with their healthcare systems. One reason is the lack of infrastructure in healthcare in developing countries [3]. This leads to minimally trained workers with equipment in clinics and laboratories that are beyond the scope of their background in healthcare. With minimal training, these workers are not able to appropriately use the technology leading to troubles when analyzing the biological samples of patients for diagnosis [3]. Therefore, when diagnostic equipment is purchased or donated to these low-income clinics, it is not used appropriately and therefore does not serve its function. Additionally, when the equipment breaks there is little incentive to repair it. This is due to the fact that there is little money to repair the technology as well as minimal education as of how to. This misuse and common failure of medical equipment leads to mistrust of these clinics as it leads to a considerable amount of erroneous results for patients. With a cost-effective, simple yet sensitive diagnostic device, many of these issues could be resolved [13].

\section{Point-Of-Care Diagnostics}

Point-of-care diagnostics is defined as the capability of diagnosing a patient at the site of care - whether that be at a local doctor, or at home. There is a growing need for this around the world that the field of microfluidics attempts to solve.

Currently, healthcare paradigms are set-up as having different diagnosis capabilities based on the location of which the sample is being studied. A central hospital is considered gold standard for patient disease diagnosis [3]. A central hospital allows for 
a patient to receive appropriate care on-site while its location contains advanced technology for the rapid detection and determination of the sample. Additionally, an abundance of research and development takes place at a centralized hospital due to the vast amounts of money and the highly trained person working there. The next best place for disease diagnosis would be at a reference laboratory [3]. This is where biological samples are outsourced to from a local doctor's office or clinic for testing. Following a reference laboratory, would be a local physician's office [3]. Many times, a general doctor's office does not contain devices allow for rapid and accurate diagnosis. Therefore, lateral flow assay tests are commonly used. Lateral flow assays, however, are qualitative rather than quantitative, which does not allow for a doctor to gage the degree of severity.

The next best technology is an emergency first responder, where those that are in the field can test for biological threats in the water or air [3]. For example, an emergency first responder device could be dipped into a stream to see if it is safe for drinking or if there are toxic contaminates. However, these devices are not usually sensitive enough to detect a lethal dose of a chemical or pathogen. Additionally, there is the category of home diagnostics (i.e. a home pregnancy test). Patients tend to prefer having the ability to be diagnosed within their own homes because they maintain a sense of privacy [3]. However, these tests are typically not as sensitive as those in a laboratory setting, so therein contains the chance of false diagnosis. Finally, those in the armed forces need disease testing available to them [3]. These people are constantly exposed to harsh conditions and there are not many options for diagnostic devices that would maintain their sensitivity in a war zone. Additionally, those in the armed forced would need to be 
able to easily carry the device as well as operate it and understand the results. This is where the concept of paper microfluidics becomes an advantageous option for medical diagnostics. There is once case recently in which a portable malaria test was developed by the United States Military for soldiers abroad [3]. This lateral flow assay allowed for a small pinprick of blood at its input and process it to see whether or not the sample was positive for malaria. Additionally, technologies that are successful within the military

would also be feasible for use in the developing world. This is due to the commonality between the environmental conditions and untrained users that the devices are aimed toward. Therefore, paper microfluidics would be successful over a wide range of locations and education levels of people.

\section{Current Technologies}

As the field of diagnostics is advancing, there are simplistic technologies that are being developed that would prove useful in low-resource healthcare situations. Two main examples of this would be microfluidics and immunoassays.

An immunoassay encompasses technologies that use antibodies or antigens in order to detect proteins or chemical substance from a sample. One example of an immunoassay is an enzyme linked immunoabsorbant assay (ELISA) [14]. Although there are several formats of the ELISA, one most often thinks of a microtiter plate an antigen sample that is attached to its surface, and is then exposed to a primary and secondary antibody with an enzyme. When the color in the well changes, this shows an immune reaction (or a detection between antibodies and antigen) and the intensity of the color is measured using a spectrometer [14]. Other common examples are Western, Northern, and Southern blots as well as immunochromatography (which implies a rapid diagnostic test 
with a lateral flow assay) [15]. Examples of these range from an at home pregnancy test to an on site lateral flow assay that tests for West Nile Virus [16]. These tests can be used at the point-of-care and tend to be purely qualitative, meaning, the test can only prove positive or negative for a particular biomarker [3]. However, the simplicity and robustness of such a device allows for its use in any healthcare environment. Additionally, many researchers wish to use microfluidics technologies as a diagnostic solution in low-resource healthcare settings [3]. Using small amounts of sample (in the microliter range), a microfluidic apparatus determines its properties. This could be through using immunoassays within the chip, using electromagnetic or thermal sciences for cell separations, and more [17]. Although this technology field hopes to eventually take what are currently large laboratory procedures and instead solve the diagnostics on a chip, this field needs to dramatically develop before most of these chips can be used in the field. For example, these chips are made on an individual basis, where materials are laser cut and milled [3]. This adds cost to the device technology, making PDMS chips not feasible for low-resource settings at this current time.

Paper microfluidics takes these two research areas and combines them together. It allows for small chip designs and required sample, similar to microfluidics, but uses inexpensive platforms and biochemical agents (like antibodies), which is similar to an immunoassay. Low-resource healthcare facilities in the developed and developing world could adopt these technologies, environmental and homeland security could use these to check for toxins, or a patient could use this at home as a private point-of-care diagnostic [3]. As long as the test remained both inexpensive and accurate, this would be favorable in numerous situations. 
The movement toward developing paper microfluidics first became well known through the Harvard University professor George Whitesides [18]. His technologies began to be adapted further by other institutions and research groups. Currently these paper microfluidic devices are still in initial development stages through characterizing flow rates, using different platforms, finding optimal detector and capture reagents, and eventually seeing the feasibility of using them on-site in the developing world [1, 18-22].

\section{Lateral Flow Assays and Immunochromatography}

When first considering the idea of paper microfluidics, it is common to think of a rapid lateral flow test strip. A common example of this is an at home pregnancy test. This in vitro test strip inputs a sample of urine that flows down a series of membranes to detect human chorionic gonadotropin (hCG), a hormone found when a woman is pregnant [23]. Pregnancy tests are known as sandwich assays. Referring to Figure 1, the urine sample goes in through a sample port and reaches a sample pad. The purpose of this pad is to promote an even and controlled distribution and rate of the sample in the immunochomatographic device. This pad typically is made out of woven meshes and cellulose fibers [23].

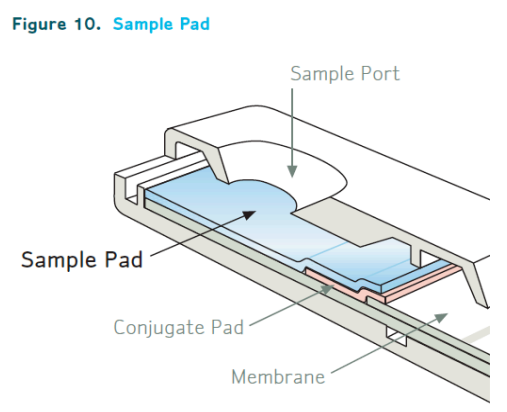

Figure 1: Sample Pad. A schematic of the sample pad in a lateral flow test strip. This is the location in which biological sample is inputted into a lateral flow assay [23]. 
As the sample moves down the sample pad via capillary action, this uniform sample is transferred to the conjugate pad. The conjugate pad is important in this device because it contains detector reagents. A detector reagent is a particle, such as colloidal gold or a latex bead, which forms a complex along with a biological sample that is to be detected, and the detector [23]. This detection particle allows for a reaction to happen to signal that the test is complete by creating a notable event - such as fluorescing for a positive result. This sample uniformly moves down the conjugate pad and as it flows, carries the detector particles with it. This membrane is commonly made of woven filters with cellulose, glass, or plastic fibers [23]. An example of the conjugate pad for a lateral flow test strip can be seen in Figure 2.

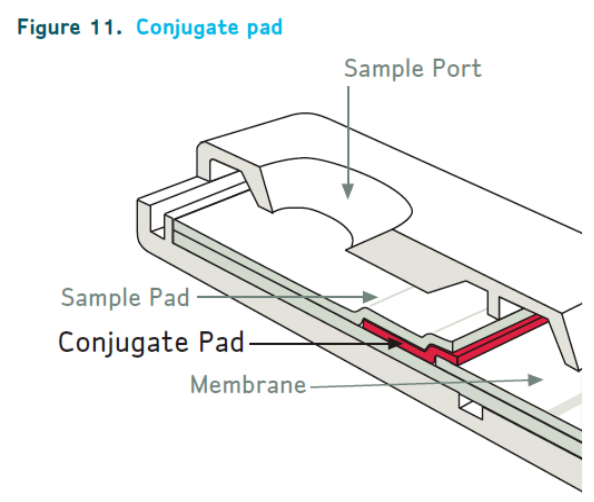

Figure 2: Conjugate Pad. A schematic of the conjugate pad in a lateral flow test strip. This is where labeled antibodies are located that will rehydrate, bind, and move along with target analytes in the inputted biological sample for a lateral flow assay [23].

The sample and detection particles reach the membrane (as seen in Figure 3), where a series of antibodies or other capture reagents are located. This membrane in a lateral flow assay is most likely made with nitrocellulose. The capture reagents tethered 
to this membrane identify whether or not a hormone or protein is present in a sample, creating an entire complex when it is identified. These reagents are commonly placed in one designated area called the detection line. When biochemically placing the antibodies or capture reagents on the membrane, it is important to know the binding mechanism of the biologics to the membrane in order to stabilize it to the surface (i.e. how nitrocellulose binds to proteins electrostatically). At this point, the detector reagents, along with the capture reagents and the target found in the sample will create and entire complex and change a color as a visualization that the protein or hormone was detected [23].

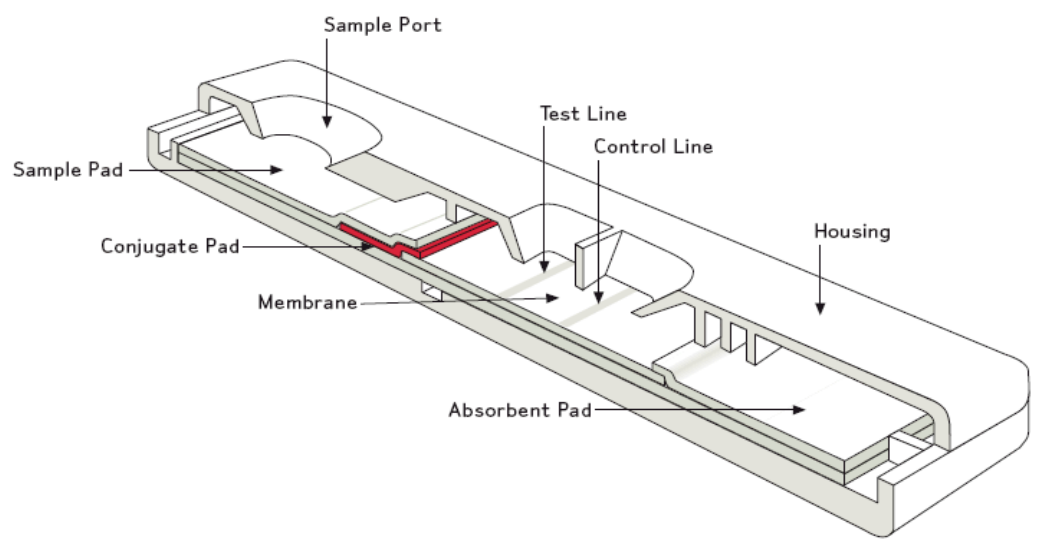

Figure 3: Entire Lateral Flow Assay. A schematic of an entire lateral flow assay with a cross-section through the plastic outer casing to show all components of a standard test [23].

Finally, the sample wicks to an absorbent pad. The absorbent pad soaks up unbound detector particles and excess sample fluid and hinders backflow onto the membrane. This is important for maintaining the sensitivity of the test. This is commonly made from cellulose filters [23]. This can be seen in Figure 4. 


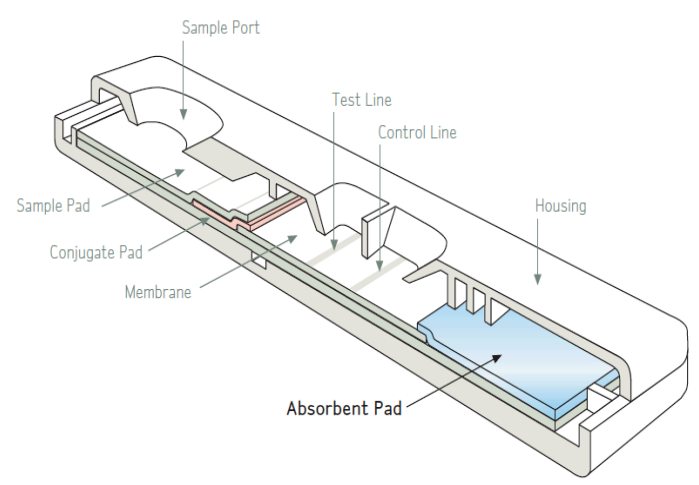

Figure 4: Absorbent Pad. A schematic of the absorbent pad in a lateral flow test strip. This region is located after the detection areas of the test and absorbs excess sample in order to prevent backflow, which would decrease sensitivity and increase the chance of an erroneous result [23].

There is also a backing commonly attached to the nitrocellulose membrane.

Proteins have a naturally affinity to want to bind to nitrocellulose. The backing of the membrane limits the amount of surface area that proteins have to adhere to the nitrocellulose. Additionally, a backing on the membrane allows for more support for the nitrocellulose, because the un-backed material has minimal tensile strength. This adhesive backing is composed of a plastic packing, adhesive, and release liner [23].

Another important aspect to consider in a lateral flow assay is whether housing is necessary. A case is important from the standpoint that it holds the entire test together into one sleek device, as well as the fact that it limits exposure to outside chemical and biological agents, which could alter the accuracy of the test. It will not only limit outside elements from altering test results, but it will also contain the patient sample [23]. 


\section{Paper Microfluidics}

Paper microfluidics, similar to a lateral flow assay, comprises of a paper base platform, capture reagents, detector reagents, conjugate pads, absorbent pads, and something different - a bridging layer [19, 23, 24].

\section{Paper Platforms}

Currently, a common paper platform material used in paper microfluidics is cellulose. However, nitrocellulose and nylon are alternative materials that are being researched and used - with this particular project focusing on the former. The paper platform represents the membrane aspect of the lateral flow assay [23].

The pore size of the paper makes a difference for the wicking of the biological sample through the entire apparatus. If the membrane has a larger pore size, this allows more biological sample to wick through, creating a quicker test. Although a short turnaround time for diagnosis is optimal, if a test is too short this can inhibit the ability of a capture reagent to attach onto a target molecule, therefore leading to erroneous results [23]. With a smaller pore size, this creates not only a longer test time, but depending on the sample, may not allow for any of the necessary target molecules to seep through the pore for detection. Once again, this could create incorrect results and is an important factor in considering platforms for paper microfluidics.

Nitrocellulose was chosen as an alternative material to that of cellulose for a number of reasons. Primarily, nitrocellulose has an affinity for binding to capture reagents, which is important for creating a paper microfluidic device. Nitrocellulose's primary binding mechanism is electrostatic [23]. This is determined by its surface area which is further determined by its pore size, porosity, thickness, and structural characteristics [23]. The electrostatic binding mechanism happens due to dipoles in the 
paper itself to that of the peptide bonds in the reagent. Additionally, nitrocellulose would not contaminate the sample that is wicking through the membrane, and would therefore maintain a sensitive and accurate result as the target molecules reach the control line.

\section{Bridging Materials}

Additionally, paper microfluidic devices that are three-dimensional require the bridging of the paper layers to allow for constant wicking of the biological sample throughout the test platform. Since the membrane material used is nitrocellulose, the bridging layer would be of the same material in order to maintain consistency throughout the chip. The process of developing this bridging layer is further explained in the Methods section.

\section{Capture and Detector Reagents}

Detector reagents are a vital part of a paper microfluidic apparatus. This is the part of the target molecule - capture regent - detector reagent complex that together creates the chemical reaction to visually prove to the patient or clinician that the test results are positive. As the detector particles that are located on the conjugate pad are exposed to the liquid sample, they flush out and eventually wick onto the detection membrane in the device. The detector particles and sample eventually reach a set of capture reagents on a control line and create an entire structure that allows for the test to turn a color for a positive result [23].

An important component of a detector reagent is that it must be spherical in shape to help with its attachment to the capture reagent and biomarker [23]. Additionally, they should all relatively be of similar size. This is due to the fact that as the fluid sample wicks down the lateral flow assay from the sample port to the detector pad to the 
membrane, the detector reagents must evenly flow from the standpoint of sensitivity. Larger and smaller particulates will move along the membrane at different time points and therefore the test may not properly fluoresce if the particular biomarker is contained in the sample that it wishes to look for. There are many types of detector reagents that can be used in a lateral flow assays. Some examples are gold colloidal particles, other metal colloidal particles, magnetic particles, dye sacs, latex beads, fluorescence particles, and enzyme conjugates [23].

\section{Fabrication}

The fabrication techniques that are used in paper microfluidics are inexpensive and simple to replicate. These chips, known as $\mu$ Pads, can initially be made as a twodimensional or three-dimensional design. A two-dimensional design uses a single membrane of the paper-based platform, whereas a three-dimensional apparatus uses multiple layers that are adhered to one another [19].

These designs are initially sketched out using a software design program such as AutoCAD or Adobe Illustrator. From there, the designs are directly printed onto the membrane of choice using a wax-based printer. The printer melts colored wax blocks that create ink and dispense it across the page [21]. As the ink quickly cools, this forms a wax layer on the surface of the platform. This provides the design of the chip, including its hydrophobic channels. These channels are what a biological fluid will wick down during testing. The printer that will transfer the CAD design onto the membrane is a XEROX wax block printer. This printer distributes fifty to sixty micrometer diameter ink droplets

onto the paper [21]. The wax has a melting temperature of $120^{\circ} \mathrm{C}$, which the printer heats the ink blocks up to during the printing process [21]. 
The wax must impregnate the entire membrane in order to maintain the appropriate barricade of the hydrophobic channel [21]. Without the wax fully infused throughout the membrane, the biological sample will naturally wick through any part of the paper platform. This would not allow for the intended flow paths of the device. In order ensure the permeation of wax in the apparatus the printed pieces of the chip are placed in an oven at a specified temperature and time period (see the Methods section). As the wax melts into the pores of the paper, it diffuses in both the lateral and vertical directions. This dispersion takes the once sharp image and alters its defined edges. Therefore, the channels that the fluid wicks through decreases in size, and the barriers widen and seep into the wax-free areas [21]. An example of this melting can be seen in Figure 5, below.

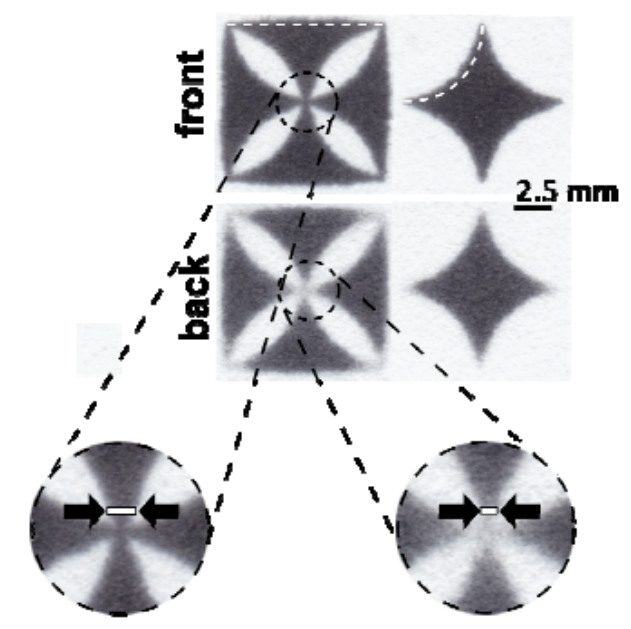

Figure 5: Wax Reflow. The reflow of wax post-melting the paper-based designs. The spreading of the wax causes a less distinct barrier between wax and wax-free areas on the paper, and how the spreading is noticeably different from one side of the paper to the other (i.e. top of the paper and bottom of the paper). This shows not only the spreading of wax laterally, but vertically as well [21]. 
The effects of lateral melting can be accounted in the design using Washburn's equation (Equation 1) [21].

$$
L=\sqrt{\frac{Y D t}{4 \eta}}
$$

In this case, $\mathrm{L}$ is the distance into the porous nitrocellulose, $\eta$ is viscosity, $\mathrm{D}$ is pore diameter, $\mathrm{t}$ is time, and $\mathrm{Y}$ is the surface tension [21]. Additionally, the width of the hydrophobic barrier is related to the width of the printed line (Equation 2) [21].

$$
W_{B}=W_{p}+2 L
$$

$\mathrm{W}_{\mathrm{B}}$ is the width of the barrier, $\mathrm{W}_{\mathrm{P}}$ is the width of the printed line, and $\mathrm{L}$ is the distance that the wax spreads from the edge of the line [21]. Therefore, the width of the hydrophilic channel is calculated using Equation 3 [21].

$$
W_{C}=W_{G}-2 L
$$

In this case, $\mathrm{W}_{\mathrm{G}}$ is the width between the two printed lines and $\mathrm{W}_{\mathrm{C}}$ is the width of the hydrophilic channel [21].

\section{Biochemistry}

This thesis compares the ability to detect VEGF in sample on a two-dimensional nitrocellulose chip using both antibodies and aptamers as possible capture reagents. Comparing the capabilities of aptamers to antibodies would allow for possible alternatives in the biochemistry involved with a lateral flow assay or paper microfluidic platform. 


\section{Vascular Endothelial Growth Factor}

VEGF, or, vascular endothelial growth factor, is a signal protein found in the body as new vasculature is formed in the body [25]. This particular growth factor is found in high concentrations for a multitude of human diseases and conditions. Although common for a wide-range of health issues, this protein is a great starting point for developing a wide array of diagnostic devices for many disease types.

There are many growth factors that are apart of the VEGF family, namely VEGFA, VEGF-B, VEGF-C, VEGF-D and PIGF (placenta growth factor) [25, 26]. However, because VEGF-A was the first protein discovered, it is commonly just referred to as VEGF [27]. VEGF-A is the overall biomarker that is of interest for this thesis particularly focusing on $\mathrm{VEGF}_{165}$ isoform [28]. This particular isoform is of interest because of its abundance in angiogenesis (or vessel growth), which is implicative of possible tumor growth in a physiological system [29].

VEGF activity is commonly seen on endothelial cells, but can also be active on macrophages, neurons, cancer cells, and kidney epithelial cells [27]. Although this signaling protein is found in all humans, higher concentrations are typical in those with cancer, tuberculosis, macular degeneration, and POEMS syndrome [4, 26, 27, 30-33]. This is because in order to feed these diseases, an increase in blood vessels develops to provide nutrients to the affected area (i.e. blood to a growing tumor). Many of these diseases plague both the developed and developing world - implying the importance of understanding how to create a less expensive and simplistic device that would be sensitive in detecting this signaling protein.

In low-resource areas, people will more commonly have to deal with tuberculosis or cancer than POEMS syndrome or macular degeneration, though detecting any of these 
would be advantageous. The developing world is more commonly plagued by infectious diseases than by noncommunicable ones, and of those, tuberculosis is one of the top three that affect these areas [3]. However, there have been numerous efforts put forward for detecting tuberculosis using rapid diagnostic tests, and using more specific biomarkers as compared to VEGF [3]. However, the effort put forward toward the detection of cancer in the developing world is minimal. Yet, numbers of cancer patients have begun to skyrocket in recent years and there is a need to help detect this disease with lower cost platforms $[11,34]$.

In particular, $\mathrm{VEGF}_{165}$ has two domains. One is the receptor-binding domain (RBD), and the other is the heparin-binding domain (HBD) [29]. All forms of VEGF have a receptor-binding domain, but it is $\mathrm{VEGF}_{165}$ that only has the $\mathrm{HBD}$, which distinguishes it as an isoform [35]. Commonly the receptor-binding domain of VEGF binds to tyrosine kinase receptors and will trigger the cellular pathway that leads to angiogenesis [35]. That is why with the development of drugs to treat cancer and macular degeneration, antibody fragments are created that bind to the RBD of VEGF to inhibit the growth factor from instead binding to cells and creating vessel growth [35].

\section{Cancer}

Cancer is defined as an uncontrolled growth of abnormal cells in the body. Initially, a healthy human cell multiplies when needed for finding sickness, or growth. However, the body also knows to regulate the cells that multiplying, and to stop them from doing so [36]. However, with cancer, cells continually grow without regulation, and very few die. Cancer can occur in almost all organs and tissues within the body. Because 
there is such a wide array of cancer types, there is not one overall symptom for the chronic disease. Additionally, its cause is unknown.

Currently, cancer is diagnosed in a variety of ways. This spans from taking a biopsy of a tumor, to blood tests, bone marrow biopsies, chest x-rays, complete blood counts, computed tomography (CT) scans, and magnetic resonance imaging (MRI) scans $[12,27,34,37]$. These diagnostic tools are used depending on the location and type of cancer that is suspected.

Cancer treatments are also varied depending on the type and what stage the cancer is in. Cancer is separated into stages I through IV. The higher number implies that the tumor has grown larger, or the cancer has spread from the area in which it first appeared to other organs. With that said, the main three types of treatment out there currently are radiation, chemotherapy, and surgery $[12,27,34,37]$. These therapies are often combined for one patient as well. Note that these treatments are usually costly and not widely spread throughout the developing world.

As previously stated, infectious diseases, particularly HIV, malaria, and tuberculosis, plague the developing world [3]. However, much recognition has been gained, forming a large-scale effort to diagnose and treat these diseases abroad. There is now a growing burden for non-communicable diseases, such as cancer, in low-income countries $[11,12,34,37]$. Yet, health systems in the developing world are designed to cope with infectious disease and do not have the resources to deal with chronic care [12].

Previously, non-communicable diseases were commonly associated with nations that were developed. However, it has been estimated that cancer fatalities are $74.5 \%$ in low-income countries versus $46.3 \%$ in high-income areas [11]. Because of the common 
misconception that cancer affects developed nations more frequently, only five percent of global funds dedicated to cancer research and treatment are used in the developing world [12]. On the other hand, it is difficult to come up with treatments in these areas when there is no standardization of which to diagnose and treat cancer. Although middle and high-income areas have successful plans for approaching cancer, these are not applicable in low-income areas due to lack of communication, education, and resources [3]. It is interesting to see that worldwide, breast cancer in women and lung and bronchus cancer in men have the highest number of estimated deaths [11]. After recognizing the desperate need to treat cancer in both the developed and developing world, it is obvious that these two seemingly different worlds can coalesce to create one set of affordable, accurate, and successful solutions for the treatment of this disease.

Although detecting VEGF would only imply the possibility of cancer in a patient rather than prove it, combining this detection with various other biomarkers in a multiplex assay could eventually allow for an alternative, simplistic, and less expensive design [36]. Earlier and inexpensive detection of cancer would also allow for efforts toward alternative techniques for the treatment of cancer.

\section{Antibodies}

In present day, antibodies are commonly used to detect proteins in biological samples for lateral flow assays [23]. An antibody, or immunoglobulin, in a physiological system is used for an immune response [38]. These immunoglobulins are made to particularly identify a foreign particulate, called an antigen. As antibodies are typically modeled in a Y-shaped formation, the tips of the bifurcated ends are what bind to an antigen [38]. This particular binding site on the antibody is what is called a paratope [38]. 
In the field of diagnostics, antibodies are developed from an array of species that are directed against very specific antigens. As they are developed, they can be used in an array of immunodiagnostic applications such as ELISA, Western blots, immunofluorescence, and so on $[15,16,39]$. Up to this point in time, antibodies are the main source of detection in lateral flow assays (i.e. a pregnancy test) [23].

\section{VEGF Antibody}

Anti-VEGF antibody is what specifically binds to VEGF. This monoclonal antibody has famous application outside of the human body. Bevacizumab is an anticancer drug that is used to block angiogenesis by inhibiting VEGF-A from stimulating vessel growth [40].

Seeing that this antibody has already been used as a metastatic cancer therapy treatment, this implies that VEGF and its monoclonal antibody are important to understand and use further in both cancer and diagnostics [40].

In using anti-VEGF antibody in diagnostics, the biochemistry would be set up similarly to an ELISA sandwich assay. What this means is that a sample of VEGF would be inputted to the test and combine with a detector particle. In this thesis, the detector particle is gold tethered to anti-VEGF antibody. Then, the two would flow down the test channel and be captured by another set of anti-VEGF antibodies that are immobilized to the test platform. This is illustrated in Figure 6, below. 


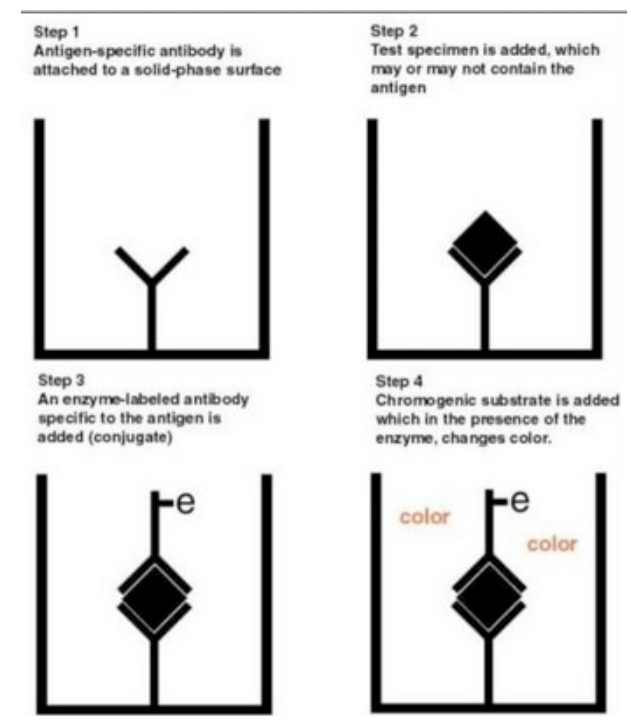

Figure 6: Sandwich ELISA. The demonstration of an immobilized antibody on a test platform. A capture antibody is immobilized onto a surface, then a target protein in sample and a detector antibody bind, creating a sandwich ELISA [41].

In other words, the two antibodies (in this case anti-VEGF and anti-VEGF conjugated with gold nanoparticle) sandwich the specific molecule, or VEGF, in the middle.

\section{Aptamers}

Aptamers are defined as oligonucleic acids or peptide molecules that bond to compatible biomarkers. This particular thesis will focus on the oligonucleic acids - or RNA and DNA strands. They bind to biomarkers in a sample as well as a detector reagent in the paper microfluidic chip in order to fluoresce for a positive result [42]. Aptamers have many uses in the field of diagnostics, including microarrays, microfluidics, sandwich assays, and electrochemical biosensors [29].

These manmade DNA or RNA strands were chosen by the disease or diseases that the $\mu \mathrm{Pad}$ will detect. These coded strands are available in a database called the SELEX system [42]. This is a library of single-stranded oligonucleic acids going from the fiveprime end of the sequence to the three-prime end. These linear sequences are targeted in 
the library until the particular chain regarding the aptamer of interest is selected. Then, the aptamer is amplified using polymerase chain reaction (PCR) and makes the doublehelix, reminiscent of a DNA strand. Then through transcription, the double helix is taken apart into single strands [42]. Transcription is when DNA unwinds from its helical structure and creates a complementary strand, where the tyrosine bases are then created as uracil, instead. This makes a since stranded tRNA.

The central dogma of genetics states that DNA creates RNA through transcription, and RNA creates a protein through translation, completing the entire synthesis process of the cell's peptide formation in the body. However, when PCR limits the process to only transcription, the single strands want to naturally form interactions within its own strand, pairing uracil with adenosine, and cytosine with guanine. This interaction will make for an ineffective aptamer, since it will no longer be able to appropriately bind with a biomarker in a sample. Therefore, while the strands are still being processed, they are incubated with their complemented substance of interest (i.e. their biomarker) [42]. They are then tethered to the nitrocellulose using filtration, so it creates the tethering effect. This is what will happen when fabricating the $\mu \mathrm{Pad}$. Additionally, these strands are tethered onto the paper platform at the control and test line [23].

In many cases, aptamers are a better option than antibodies for diagnostic purposes. For one, aptamers can be created completely in vitro using the SELEX process. Antibodies, on the other hand, must come from in vivo, which adds to the complication in creating and gleaning the antibodies, which brings up the price of the aliquot and lowers specificity control by the scientist. In order to produce antibodies, an immune response 
must be invoked from the physiological system. An aptamer, because it is created entirely in vitro, is screened to be very specific for its target protein [43]. And with that high control, the binding conditions can be controlled (i.e. salt concentration, temperature, and pH) [43]. Additionally, antibodies denature much more easily than an aptamer.

Antibodies must remain in a specific temperature range and be stored for a very short amount of time before denaturation. Aptamers, on the other hand, can maintain their integrity over a much larger temperature range and can be stored for a significantly longer amount of time [43]. Finally, labeling antibodies can cause a loss in their affinity for target molecules, whereas aptamers can be labeled and their binding affinity is not compromised [43].

\section{VEGF-A 165 Aptamer}

Development of VEGF aptamers has been sought after for an array of uses. An RNA aptamer has been developed (Macugen) as a drug that treats age-related macular degeneration $[28,29]$. However, for this thesis the VEGF-A aptamer is a single stranded DNA sequence that is intended to work similarly to an antibody as a capture reagent in a lateral flow assay [29]. It is immobilized to the test surface and is intended to identify and capture VEGF in sample.

VEGF-A has many different forms in the human body. There are several published aptamer DNA and RNA sequences for anti-VEGF in literature. However, after many experiments involving different aptamer sequences, it was seen that the isoform of human VEGF $_{165}$ maintained a strong attachment and provided strong visual signal to this 28 mer oligonucleotide sequence that acts as anti-VEGF [29]:

$$
\text { 5' - GCACTCTGTGGGGGTGGACGGGCCGGGT - 3' }
$$


This oligonucleotide sequence, or aptamer, is known as Vap7. This aptamer-target complex has a dissociation constant $\left(\mathrm{K}_{\mathrm{D}}\right.$ value $)$ of $20 \mathrm{nM}$ [29]. Copious amounts of VEGF $_{165}$ isoform are seen in cancer patients because it is highly expressed in angiogenesis [29]. Therefore, an aptamer that is specific for this isoform would be advantageous to use as a capture reagent in detecting cancer biomarkers.

Unlike an antibody, an aptamer made for $\mathrm{VEGF}_{165}$ can be made to attach to the HBD region of the analyte $[29,35]$. Therefore, the aptamer sequence provided can attach to either the $\mathrm{HBD}$ or RBD region of $\mathrm{VEGF}_{165}$, whereas the antibody can only bind to the RBD region [35]. In a lateral flow assay, this allows for more opportunity for detection of VEGF using aptamers as a capture reagent as compared to antibodies.

\section{Advantages and Disadvantages}

The subject of paper microfluidics is continually being researched due to the fact that the advantages of this disease detection technology far outweigh the disadvantages. Yet, there are still problems and improvements that must be made with this concept in disease detection technology.

There are several negatives that pertain to this technology. One con to this technology is that the diagnosis is purely qualitative rather than quantitative [3]. There are a wide variety of diseases that need quantitative measurement. One example would be with cancer diagnosis. Although these chips could detect whether VEGF is present in the blood, it is currently only qualitative [3]. A patient might need to know to what degree the disease is present. This would allow for those in clinics to see what approach should be taken to its treatment or a possible prognosis. Another issue is the lack of time control with the device [3]. Once a sample is in contact with the chip, it will wick due to the 
material properties of the paper with the biological sample itself. This wicking time can be controlled to an extent by designing the paper platform using particular calculations and equations - however the density of the samples themselves can vary due to water or pathogen content. A final issue is possible sensitivity tissues. Some diseases and sample types do not have a concentrated amount of a biomarker within the fluid. This diluted sample may not have enough biomarkers that enough capture reagents could form a complex with in order to signal a positive or negative test $[17,44]$.

There are many advantageous aspects with this type of disease diagnostic device. Paper microfluidics is, for one, affordable. The affordability comes in the fact that paper is inexpensive, and because its size is so small, numerous samples can be made on a sheet of paper. The affordability also comes with the entire fabrication process - where simple materials like wax ink, a printer, and a hot plate or oven is used [18]. This removes the need for specialized and expensive equipment. Another positive facet of paper microfluidics is biosafety. The device can be burned after use and releases no noxious chemicals into the environment. Likewise, these $\mu$ Pads eliminate the need for sharps in a clinical or laboratory environment. That adds to safety in the diagnosis area so there is no exchange of bodily fluids, and inhibits healthcare-related injuries [17]. These devices are also storable, because they are commonly the size of a postage stamp. This storability is great from the standpoint of shipping and transportation, as well as the fact that its lack of bulkiness allows for more room in the clinical area for patient care rather than devices. Additionally, storage is important because the devices must maintain their integrity. With paper microfluidic devices, reagents and platform materials used typically do not degrade or lose sensitivity for long periods of time. Another practicality of this device is that it is 
inexpensive, meaning that the device can be used by a multitude of people from varying socio-economic situations. These devices are simplistic in that they have one inlet for the sample, and the device changes color. This color can be interpreted from a chart, or possibly interpreted from a pre-programmed algorithm. This allows for minimal training of the user of the device and minimizes ambiguity in the results and incorrect diagnosis. Finally, this chip is advantageous in that it can be designed for testing multiple diseases at once [44]. By attaching multiple types of capture reagents, this can allow for a multitude of biomarkers to be detected in a patient sample to help assess the medical issues that they are experiencing.

This thesis presents the development and testing of a paper microfluidic chip. In the following section, the methods are presented and discussed. Following, the results are reported and analyzed, with the discussion proceeding. Finally, the conclusions and future directions that stemmed from this research are considered. 


\section{Methods}

This thesis comprised of three main components - characterization, development of a three-dimensional paper microfluidic chip, and the implementation of biochemistry using antibodies and aptamers.

\section{Characterization}

As indicated in the Introduction, this thesis focuses on the detection of VEGF in sample using a paper microfluidic assay with a nitrocellulose-based platform. In order to initially start this project, several types of nitrocellulose paper were tested for their ability to wick through different designs impregnated onto the paper and its pore size. In order to further develop this diagnostic technique, the paper and chip designs needed to be analyzed in order to create an optimal chip for detection.

\section{Channel Widths and Wax Reflow}

Initially it was important to determine the capabilities of the XEROX Phaser $8560 \mathrm{DN} / 8570$ (wax block printer) in regard to its control and spreading of ink on the nitrocellulose paper. Although it is known that the wax printer distributes $50 \mu \mathrm{m}$ to 60 $\mu \mathrm{m}$ diameter droplets onto paper [21], it was necessary to clarify if the channel widths were similar on the paper platform as to the designs created on CAD, or to see if spreading of the wax occurred. Therefore, a series of circular and linear patterns were developed using SolidWorks. For the circular patterns, a multitude of inner and outer radii were designed to create an array of circular channels. Additionally, lines were designed that were five centimeters in length with widths of $0.01 \mu \mathrm{m}, 0.03 \mu \mathrm{m}, 0.04 \mu \mathrm{m}$, $0.05 \mu \mathrm{m}, 0.1 \mu \mathrm{m}, 0.5 \mu \mathrm{m}, 1 \mu \mathrm{m}$, and $5 \mu \mathrm{m}$ (these designs may be found in Appendix $B$ ). 
There were many designs that needed to be fabricated and tested in order to implement the best channel distances for the most accurate and sensitive disease diagnostic devices possible. In Yao Lu's paper Fabrication and Characterization of Paper-Based Microfluidics Prepared in Nitrocellulose Membrane by Wax Printing he tested channel widths of $100 \mu \mathrm{m}, 200 \mu \mathrm{m}, 300 \mu \mathrm{m}, 400 \mu \mathrm{m}, 500 \mu \mathrm{m}$, and $600 \mu \mathrm{m}$. This testing can be seen in Figure 7 [45]. He noted that the $200 \mu \mathrm{m}$ and $100 \mu \mathrm{m}$ channels were too small to allow for the analyte $\operatorname{IgG}$ to wick through. However, it was of further interest to determine how small the channel could possibly be designed before the liquid sample would no longer be able to wick through. Additionally, the paper noted that the minimum line thickness for a hydrophobic barrier is $60 \mu \mathrm{m}$, which fits within the specifications of the XEROX printer (due to its minimum thickness of printing).

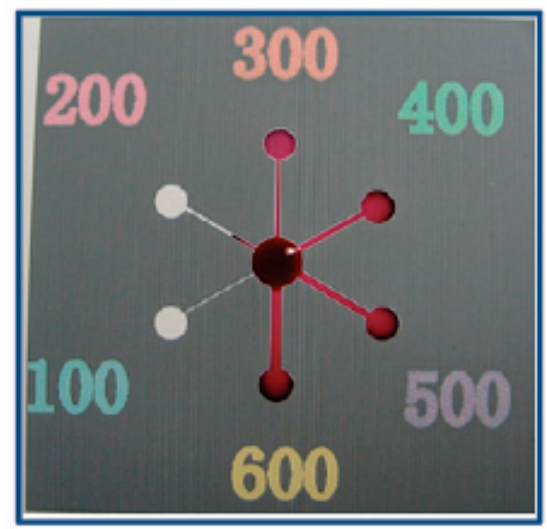

Figure 7: Varying Channel Thickness Yao et. Al. Varying channel distances (in $\mu \mathrm{m}$ ) to find appropriate widths for the wicking of sample. Note that the $100 \mu \mathrm{m}$ and $200 \mu \mathrm{m}$ channels did not allow for the inputted sample to travel through these channels [45].

This idea was redesigned (to later be printed via the wax printer) to test channel thicknesses of $225 \mu \mathrm{m}, 250 \mu \mathrm{m}, 275 \mu \mathrm{m}$, and $285 \mu \mathrm{m}$. The dimensioned design, developed in AutoCAD, can be found in Figure 8. In Figure 9, a dimensionless drawing is presented with a black background - where the black represents wax. This was initially 
printed onto standard 8.5 " by 11 " printing paper in order to see if the printer was even capable of producing these smaller channels, then melted in an oven at $150^{\circ} \mathrm{C}$ for thirty seconds to see if the wax would reflow and block the small channels. From there, food coloring was inputted into the middle circular area to see if it would wick to the four end regions.

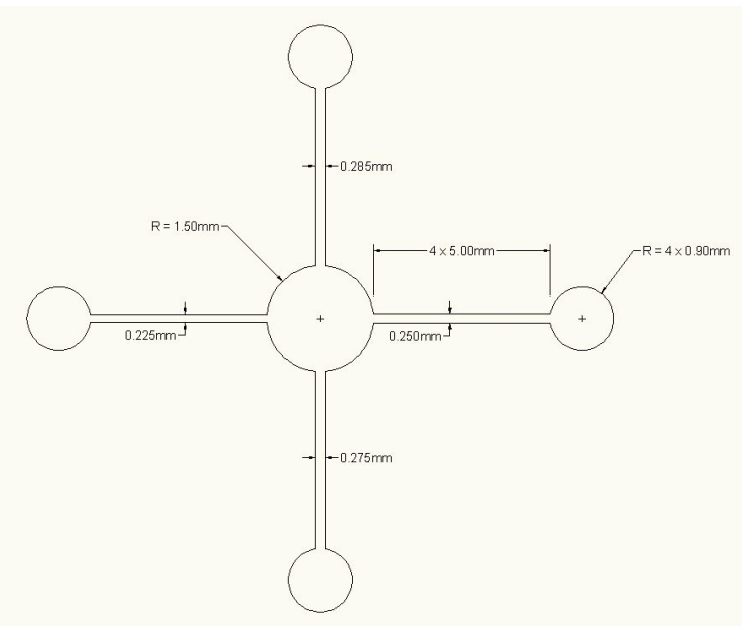

Figure 8: Dimensioned Drawing for Channel Thickness. A dimensioned drawing to study channel thicknesses of $225 \mu \mathrm{m}, 250 \mu \mathrm{m}, 275 \mu \mathrm{m}$ and $285 \mu \mathrm{m}$ as a proof-of-concept to see the capability of liquid flowing through small channels and to observe possible wax reflow on the membrane itself.

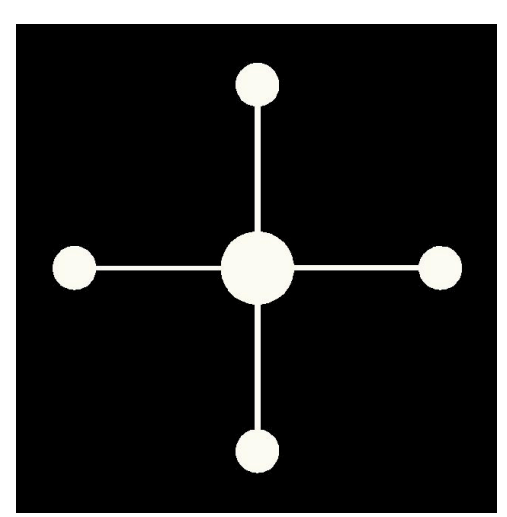

Figure 9: Wax representation of Channel Thickness. A representation of how a chip containing channel thicknesses of $225 \mu \mathrm{m}, 250 \mu \mathrm{m}, 275 \mu \mathrm{m}$ and 285 $\mu \mathrm{m}$ would be printed - where the black area represents what would be printed in wax, and the white area would be exposed membrane. 
Initially, a $0.45 \mu \mathrm{m}$ pore size roll of nitrocellulose was purchased due to its common usage in referenced literature as a paper microfluidic platform. A multitude of linear and curved designs that fit onto a 1" by 1" square were designed on AutoCAD and then printed onto the nitrocellulose paper using the wax block printer. The designs were cut out from the sheet of paper into their outlined squares. The printed wax was then impregnated into the paper's thickness via a hot plate which the chip was placed upon for two minutes at $75^{\circ} \mathrm{C}$. The same process was replicated onto 4 " by 6 " bibulous paper (chosen due to its ability to dramatically wick fluids). Then, the two paper types were exposed to a droplet of water with green dye and its wicking down the channels was observed and compared.

\section{Optimal Channel Characteristics}

Several other pore sizes of nitrocellulose membrane were purchased and used for further characterization. Initially, channel thickness was characterized, observing the differences between $0.8,1.6$, and 2.4-millimeter channel widths on the same sheet of paper. This was done using a nitrocellulose membrane referred to as HF 135. Seven designs were created on AutoCAD, printed on the wax block printer, and melted in an oven at $150^{\circ} \mathrm{C}$ for three minutes. Green food coloring combined with water were inputted into the chip and wicking time and visual determinations were made regarding the capabilities of channel widths, lengths, and curves. This was used to determine a general optimal channel width.

\section{Design Combinations and Wicking Times}

Further characterization was done in order to understand five topics regarding paper microfluidic properties and analyzed their effect on wicking time analyzed by using 
a multi-way Analysis of Variance on Minitab. The considerations evaluated on twodimensional chips were porosity, size, width, curvature, and backing. For each of these factors, two categorical levels were examined (creating $2^{5}$ or 32 combinations) in order to see the final wicking time. Two categorical levels intends that there are two factors being looked at - i.e. if the paper has more porous or less porous, a narrower channel width or a wider channel width, and so on. A faster wicking time would lead to a faster diagnosis for disease in a lateral flow assay. However, if the wicking time were too fast, this would not allow for a sensitive enough detection of VEGF in a sample, because the capture reagent would not have a sufficient amount of time to bind to VEGF in sample. Therefore, the results generated the factor level combinations desired for the most optimal wicking times was analyzed with a $10 \%$ overall significance level. An Analysis-of-Variance (ANOVA) statistical method was done in order to analyze the five different characteristics and their wicking time through a two-dimensional platform.

Two-dimensional nitrocellulose chips were designed and fabricated in a variety of ways for the purpose of fulfilling this project. In this project, the experimental units were the chips and the response was the amount of time it took for an inputted amount of dyed water to wick through the entire chip. This test was a five-factor completely randomized design. The five factors (porosity, size, width, backing, curving) at two levels each allowed for 32 possible treatments for this project. The first factor, porosity, was a categorical treatment where the levels were, "hi" and "lo". Nitrocellulose paper's porosity is described by Millipore through its capillary flow time (measured in seconds for the liquid to travel 4 centimeters). Therefore a higher number describes a longer time it takes for a solvent to wick through the paper, intending lower porosity. Therefore, "hi" 
described the HF (high flow) 135 membrane paper (less porous) and "lo" described the HF 75 membrane paper (more porous) for non-backed nitrocellulose membrane.

However, unbacked membranes were also analyzed, and in that case "hi" described the HF 180 membrane paper and "lo" described the HF 135 membrane paper. The next factor was referred to as size. Size implies the overall length of the channel of the chip. In this project, the lengths of this chip were "long" or "short" where long was a 63 millimeter length channel, and short was 31.5 millimeters. Additionally, the curving factor was whether the channel was designed linearly or as a single curve. Likewise, the backing factor was whether the nitrocellulose stood alone or was adhered to a plastic film on one side. Finally, width took into account whether the channel was 1.6 millimeters (narrow) or 3.2 millimeters wide (wide). All of these factors are categorical and the response was qualitative. This allowed for a multi-way analysis of variance in order to analyze how the five factors (and their interactions) affect the time it takes for the dyed solvent to wick through the chip.

Two replicates were completed per combination and all the tests were completed in a random order. The order in which these treatments were randomly assigned was by inputting the thirty-two different combinations into Microsoft Excel on two separate spreadsheets. The five treatments were inputted into separate columns and the thirty-two different combinations were inserted. In a sixth column the function "rand()" was implemented for all thirty-two adjacent rows. Following that, all of the data in the six columns were selected and the "Sort A-Z" command was chosen in order to randomly assign the treatments. These randomized data entries are listed in Appendix A. 
All thirty-two combinations of chips were designed using AutoCAD and printed on a Xerox 8560DN wax-block printer. This printer deposited wax onto the paper (similar to an inkjet printer). These chips were then placed in an oven at $150^{\circ} \mathrm{C}$ for three minutes. This allowed the wax to fully impregnate the chip and create hydrophobic barriers around the channel. This is so the fluid is forced down the channel when it is introduced to the chip. The chips were then removed from the oven and allowed to cool at room temperature for several minutes. The experimental order followed the random order displayed in the designed Excel document. In order to see the movement of the liquid through the chip, a combination of green food coloring and distilled water were combined to make an opaque solution. The liquid would then be captured using a dropper and placed onto the input channel of the chip. From the moment that the dye drop was released was when the stopwatch to measure time was started. The end point to evaluate wicking time was when the dye reached the very end of the chip and all of the exposed (non-waxed filled) nitrocellulose had the dye wick through its entirety. Time was measured in seconds.

The ANOVA test was run using the general linear model in Minitab, a statistical analysis computer program. Since there were two replicates done during experimentation, all of the relevant interactions between the five factors were analyzed. Once the general linear model ANOVA test was selected in Minitab, the following specifications were selected:

- Response: Time

- Predictors: Backing, Curving, Porosity, Size, Width (and all possible interactions indicated using the '!')

- Four-in-One Residual Plot

- Cook's Distance 
The four assumptions that were considered when analyzing the data were normality, constant variance, independence, and model adequacy. These four assumptions are commonly analyzed in statistics in order to determine the viability of the test results in order to see if the response of the test result is significant. This thirty-two combination chip experiment was repeated twice and all results were analyzed.

\section{Wicking Time - Conjugate Pad vs. Membrane Paper}

Additionally, further characterization was done involving wicking time. Four simple linear channels were designed that involved using a conjugate pad versus four that had none at all. The drawing of these four channels with and without conjugate pads may be found in Figure 10. These were designed once again in AutoCAD and printed on the XEROX 8560DN printer and melted in the oven for three minutes at $150^{\circ} \mathrm{C}$. This step was taken in order to see how the conjugate pad affected the time difference for detection. This step was further analyzed for time differences when a three-dimensional chip was designed as well. By recording the wicking time in the two experiments, standard deviations and average times were calculated. This showed if there was or was not a major difference between different conjugate pads or different parts of a nitrocellulose membrane. From there a two-tailed t-test was performed to compare the experiments in order to see if there was a significant difference in time between using and not using a conjugate pad. 


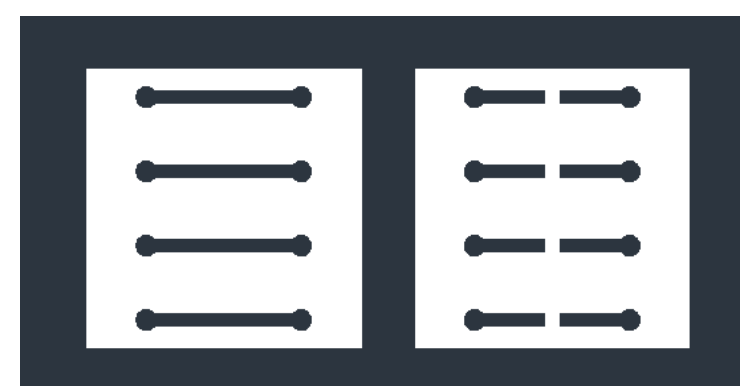

Figure 10: Conjugate Pad vs. No Pad. Designs of two experiments containing channels comparing the time it takes for liquid to flow from one side to another. The left involved no conjugate pad and the right was of the one with a conjugate pad.

\section{Imaging}

Paper was also analyzed visually in order to see how nitrocellulose paper looked at extremely different pore sizes and when it was impregnated with the wax ink. This was done using a tabletop Hitachi scattering electron microscope (SEM). Initially, a small piece of $0.45 \mu \mathrm{m}$ pore size nitrocellulose was placed onto a small podium and adhered using double sticky back tape. In was then measured that the sample sat at a onemillimeter distance away from the reader of the SEM. It was then placed into the SEM and the device was turned on. When using the SEM it pulled a vacuum and the sample was left uninterrupted until the machine was fully turned off. SEM images were taken at an area of different objectives (which will be demonstrated in the Results and Conclusions sections). This was also repeated for a piece of nitrocellulose that had both wax and did not have wax in order to determine if a difference could be seen at the seam, using the HF 135 membrane. Images simply with and without wax were also imaged using the HF 135 membrane.

\section{Development of a Three-Dimensional Paper Microfluidic Chip}

A three-dimensional paper microfluidic chip was designed in order to create a diagnostic that would allow for a simultaneous detection. If a single biological sample 
were inputted into a three dimensional chip, it would be routed into a series of separate channels containing different analytes that could bind to different proteins found in a raw sample. Then, as the sample reached the final layer of the chip, there would be several regions that would provide visual signal simultaneously to determine if a sample were positive or negative for containing particular biomarkers. This chip was designed to have four detection regions in order to prove simultaneous detection of a paper microfluidic device could be created. In order to do this, on the bottom, or base layer, was designed in order to input a fluid sample and separate it into four equal channels that would provide equal sample amounts into the chip, and eventually for wicking up into the detection region. All path lengths that the sample would wick through were equal in order to allow for simulatenous detection.

\section{Membrane Design and Development}

Each nitrocellulose paper layer was designed in AutoCAD and printed using a Xerox 8570 , as well as a XEROX $8560 \mathrm{DN}$ printer. This printer, rather than using ink, melts different colored wax blocks and deposits them onto the paper where the wax quickly dries, being exposed to a much colder room temperature. Both backed and unbacked nitrocellulose paper were printed onto. Backed nitrocellulose (meaning that the nitrocellulose paper had a plastic film adhered to its surface) was directly fed through the printer after being cut to size (due to the fact that the paper does not appropriately fit into the printer). Unbacked nitrocellulose, in this thesis, was taped down on all four sides onto 4" by 6" bibulous paper and fed through the printer then the outlines of each chip were cut out from the overall piece of nitrocellulose. Post-printing, the paper with the designs 
were placed into an oven at $150^{\circ} \mathrm{C}$ for three minutes (or until the wax fully impregnated the paper). They were then removed and allowed to cool at room temperature.

There were four main designs that were developed and fully tested in this thesis project. All were ideas that attempted to carry out simultaneous detection of a single sample at four detection wells. Additional designs that were not tested may be found in Appendix B. The first two designs tested were essentially the same. The base layer is the location in which sample is inputted. The only difference between the first and the second design are that the base layer is extended in the second design from the first. The first design can be seen in Figure 11 and the second in Figure 12, where the designs with the white background represent the nitrocellulose membrane layers embedded with wax, and the designs with the black background represent the laser cut double-sided tape.

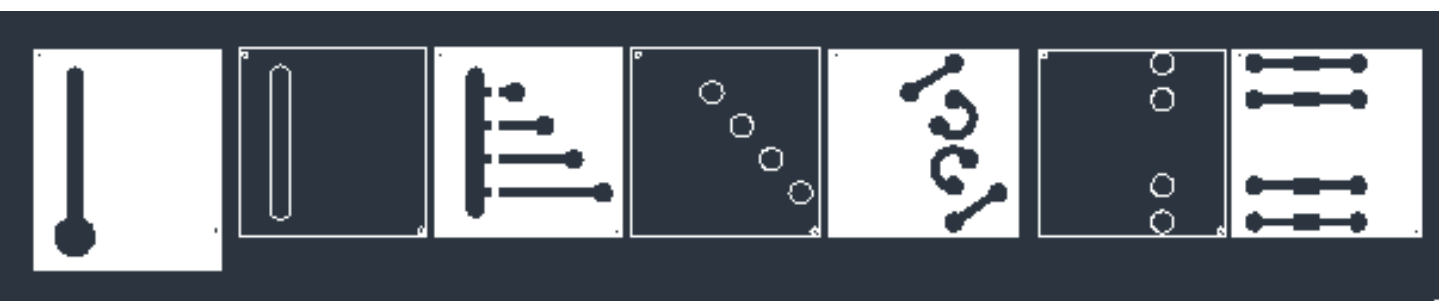

Figure 11: Design Iteration 1. Three-dimensional paper microfluidic chip design where the white background layers represent the nitrocellulose membrane embedded in wax and the back background layers are pieces of laser cut doublesided tape. The layer assembly goes from left to right where the initial piece remains on the bottom and works its way upward.

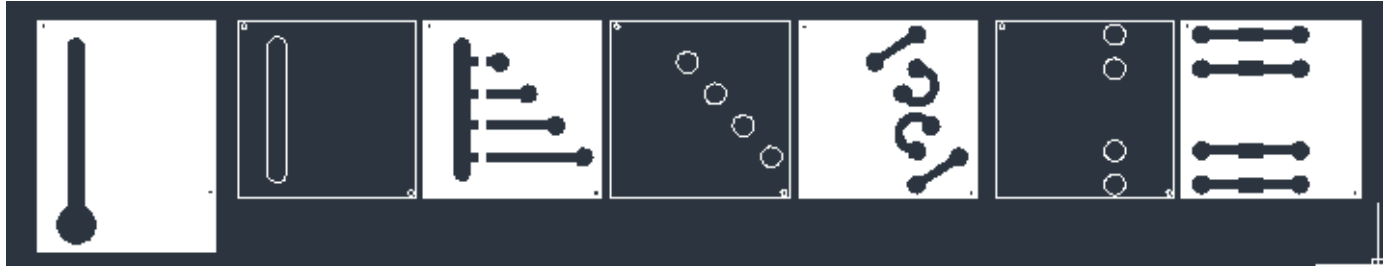

Figure 12: Design Iteration 2. White background layers represent the nitrocellulose membrane pieces embedded in wax and the back background layers are pieces of laser cut double-sided tape. The layer assembly goes from left to right. 
A third design was developed in AutoCAD and also assembled and tested as a chip for simultaneous detection of sample. In this case there are only three layers of nitrocellulose membrane as opposed to the first two designs that had four layers. This design layout can be seen in Figure 13.

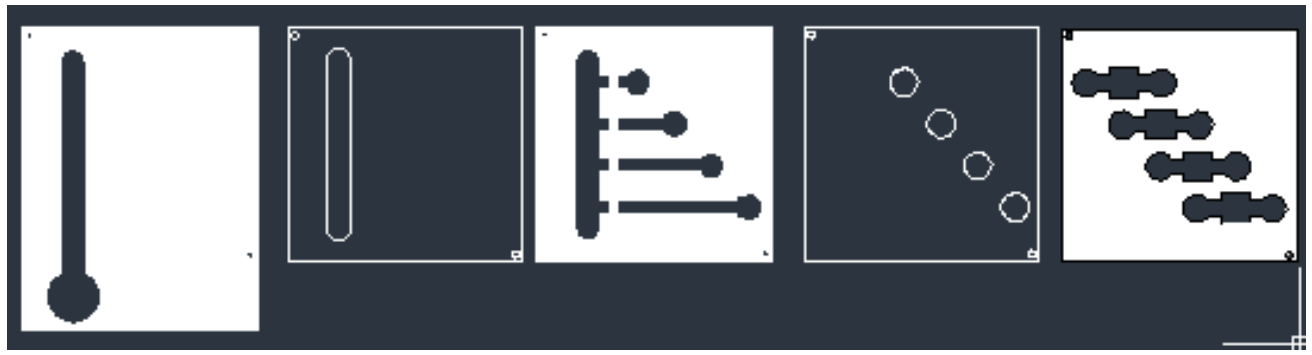

Figure 13: Design Iteration 3. Sample is inputted on the layer to the far left and works its way up to the detection layer as seen on the far right. The layers containing white background represent nitrocellulose membrane with wax and those with black background are laser cut tape layers.

The fourth and final chip tested for simultaneous detection can be found in Figure 14.

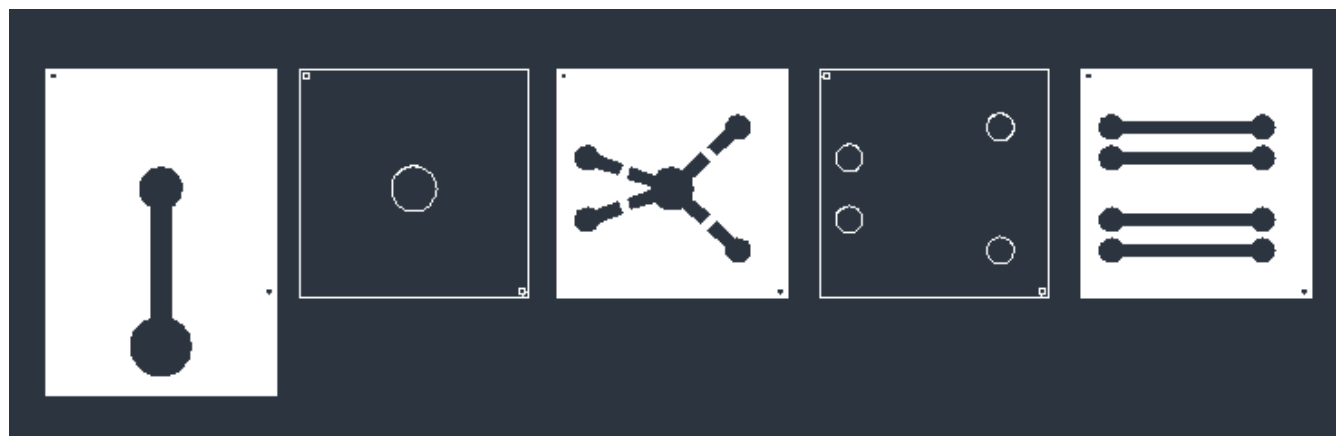

Figure 14: Design Iteration 4. The layers containing white background represent nitrocellulose membrane embedded in wax and those with black background are laser cut tape layers, and are assembled from left to right.

\section{Tape Development}

Additionally, layers of tape were designed in AutoCAD. These were then

implemented onto a computer that controlled a laser cutter and would read the design and implement it. Laser cutters commonly read AutoCAD designs in red, so the layers were set to that color in order for it to cut out the shapes that were designed. Like using a 
regular printer, the designs were "printed." The laser cutter settings were to cut 0.5 millimeter thick mylar. Each process was done twice to ensure the entire width of the tape was cut through. The tape itself was double-sided and provided with one side covered in a waxy layer. The other exposed tape side, while being laser cut, was covered with a Reynold's parchment paper. This tape was placed into the laser cutter and taped down using masking tape, in order to ensure level cutting on the tape, as a vent which provides air into the apparatus, will not make the sample move around in the machine.

\section{Bridging Layer}

In order to have an inputted sample wick through the layers of the chip, a bridging layer was developed. Initially, a powder layer was designed using pure acetone and unbacked nitrocellulose paper. This initial powder procedure looked at using both the $0.45 \mu \mathrm{m}$ pore size paper and the HF 135 or HF 180 membrane. This mixture was ground using a mortar and pestal and dried on bibulous paper. A second powder mixture was made using distilled water and nitrocellulose paper, grinding the mixture until paper was formed, and once again dried on bibulous. A third powder was developed solely using water and nitrocellulose membrane and grinding it into a powder. While still somewhat damp, it was placed onto the nitrocellulose membrane and molded into its necessary shape. Finally, a fourth powder was created by placing nitrocellulose paper into a beaker and saturating it in distilled water and allowing it to freeze overnight at $-20^{\circ} \mathrm{C}$. The next morning it was removed and ground using a mortar and pestal. This procedure was repeated for several nights until the grains were small enough to be considered a powder (less than $1 \mathrm{~mm}$ in size). A lacquer was also considered, where the nitrocellulose paper was once again ground with a mortar and pestal with nail polish remover from the 
grocery store (containing $99 \%$ acetone). Finally, a pure paper layer was considered for the final device, where, for this thesis, small pieces of nitrocellulose were cut using scissors, an Xacto-knife, or a small specialized hole punch into a similar shape of the design of the chip, where the liquid would be expected to transfer between layers.

\section{Conjugate Pads}

Four conjugate pads were prepared from strips of fiberglass provided by Millipore. They were cut out as equally as possible in order to aid in providing consistency between the four channels. For the purpose of providing a visual signal as to prove or disprove the possibility of simultaneous detection of a three-dimensional paper microfluidic chip, the conjugate pads were dyed using green food coloring. When a water sample was placed into the input of the chip, it would reach the conjugate pad and not only show green dye at the end for a visual, but prove the use of a conjugate pad. After cutting out the conjugate pad, the pieces had one to two drops of food coloring on their surface and allowed to absorb as they were placed in plastic weigh boats. They were then kept out to dry.

\section{Chip Assembly}

The tape, bridging layer, backed and unbacked paper layers, and conjugate pad were then placed together to manufacture a chip without any additional biochemistry. This was in order to solely study the workability of a three-dimensional chip before doing detection. Both the tape and the paper had small circles designed in two opposing corners in order to help with alignment. The bottom, backed layer had tape placed on top of it. While the other side of the tape is still covered with a backing, the bridging layer was carefully placed in the openings of the cut out tape using tweezers, then the next layer of 
nitrocellulose, this time unbacked, was placed on top. For the layer that needed a conjugate pad, these were placed in their appropriate location with tweezers with the double-sided tape placed on top in order to provide permanent placement of the conjugate pad onto the paper. As the final layer of nitrocellulose is placed on top, a final layer of tape is placed on top, with a fit-to-size clear transparency as the last layer of the chip. This helps to protect the detection layer of the nitrocellulose while still being able to visualize if any detection has been made. Additionally, between the tape and the membrane on the final layer, an absorbent pad was placed at the test's end to soak up any excess sample and prevent backflow of the sample in the test.

A conceptual alignment tool was designed on Solidworks where one could align the layers of tape and paper on a podium with press fit standard size sewing needles guiding the alignment of the previously drawn holes onto the designed paper and tape. The podium can be rapid prototyped using stereo lithography or fused depositon modeling.

\section{Testing of Three-Dimensional Chip}

The chip was then tested to see wicking time to the detection region and the difference in time it took for each of the four regions. Water was inputted from a syringe until the input sample pad was filled, and the time on a stopwatch was started. Then, when the green dye could be seen at the detection region, the time was stopped and recorded. 


\section{Biochemistry}

A paper microfluidic chip contains both detector and capture reagents. Detector reagents comprise of an antibody conjugated with a nanoparticle of some sort, that when aggregated together produce a visual signal. These can be done with fluorophores, latex beads, magnetic beads, and in this particular case, gold colloidal particles [23]. Capture reagents are molecules that capture sample and are immobilized onto the membrane of the test chip. In this experiment, capture reagents are both antibodies and aptamers, which are being compared. All calculations for dilutions provided in the procedure may be found in Appendix D.

\section{Conjugating Gold to Antibodies}

Initially, 15 nanometer gold colloidal particles were purchased for conjugating to a mouse monoclonal anti-VEGF antibody that was reactive with human VEGF. This conjugation was done by initially diluting the antibody to a concentration of $20 \mu \mathrm{g} / \mathrm{ml}$ with phosphate buffered saline (PBS). The antibody was then placed in a buffer containng sodium tetraborate and $10 \mathrm{e}^{11}$ gold particles per milliliter at a $\mathrm{pH}$ of 8.5 [46-48]. The antibodies and buffer were combined and settled together for thirty minutes [46].

A nitrocellulose membrane-based chip was designed on AutoCAD and printed on a wax block printer and melted at $150^{\circ} \mathrm{C}$ for 3 minutes. This design can be found in Figure 15. This design had a sample input, where the VEGF would be pipetted into the device (labeled as 4 in Figure 15). Next (number 3 in Figure 15) a wax barrier was designed. In this area a conjugate pad containing gold-conjugated antibodies were placed. In theory, the VEGF sample in buffer would rehydrate anti-VEGF antibodies and together would wick through the rest of the chip to a detection area (number 2 in Figure 15). In this initial design, the detection area was one well where both the detection and 
control line were to be spotted down (anti-VEGF antibody and IgG). Finally, this led up to an absorbent well (number 1 in Figure 15) that would draw in excess sample from the input, and have an absorbent pad (made of cellulose Whatman paper). This prevented backflow of sample through the chip, hindering the possibility of inaccurate results.

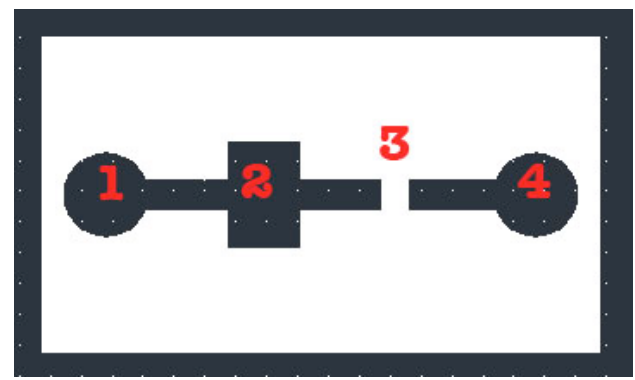

Figure 15: Small Square Biochemistry Chip. Initial 2D chip used for detection of VEGF where gold was experimentally conjugated to anti-VEGF antibodies. (1) the absorbent region; (2) is the detection region; (3) wax barrier; (4) the sample input region.

The detection antibody, a monoclonal anti-VEGF, was reconstituted from its lyophilized form using 1 milliliter of distilled water in order to yield a concentration of 1 $\mathrm{mg} / \mathrm{ml}$. Part of the sample was further removed in order to create a concentration of 20 $\mu \mathrm{g} / \mathrm{ml}$ for conjugating with gold. Then, $1 \mu 1$ of the original $1 \mathrm{mg} / \mathrm{ml}$ sample was spotted onto the nitrocellulose membrane in the detection well.

During the incubation time, the antibodies for the control line were prepared using rabbit anti-mouse IgG (immunoglobulin G) antibody, purchased from Thermo-Scientific. These antibodies were chosen as a control line antibody because their specificity allowed for them to capture the anti-VEGF antibody purchased for the test. The IgG that was attached to the paper would bind to the Fc region of the anti-VEGF antibody, so whether or not a sample contained VEGF, this region would tell the user that the test was run appropriately and nothing was detected, meaning there was no error with the detector particle (i.e. the anti-VEGF antibody and gold). A rabbit anti-mouse IgG was chosen for 
the control line because the anti-VEGF antibody was from a mouse. This meant that the IgG would have an affinity to bind to any mouse-based antibody at the Fc region.

The $\mathrm{IgG}$ antibody was diluted to a $1 \mathrm{mg} / \mathrm{ml}$ concentration using a mixture of 0.25 $\mathrm{M}$ sodium chloride $(\mathrm{NaCl})$ and $0.5 \mathrm{M}$ sodium phosphate dibasic solution $\left(\mathrm{Na}_{2} \mathrm{HPO}_{4}\right)$. These reagents were also diluted from stock using deionized water. The $\mathrm{NaCl}$ solution was measured out to $1.461 \mathrm{~g}$ using a scale, and mixed with $100 \mathrm{ml}$ deionized water. Then, 2 milliliters of $\mathrm{Na}_{2} \mathrm{HPO}_{4}$ solution was added. Its $\mathrm{pH}$ was measured using $\mathrm{pH}$ strips to ensure a value of 7.4.2.4 microliters of this buffer was removed and $0.01 \mathrm{~mL}$ of IgG antibody was combined with it into a sterile conical tube. $1 \mu 1$ of this solution was pipetted onto the nitrocellulose membrane in the detection well, along with the antiVEGF antibody. Both were allowed to dry by covering the membrane and leaving out at room temperature for two hours.

After drying, the membrane was placed in $5 \%$ bovine serum albumin (BSA) that was reconstituted from powder form using 5 grams of the BSA with $100 \mathrm{ml}$ of phosphate buffered saline (PBS) for ten minutes. This was in order to block any bare areas of the membrane to remove any chance of non-specific binding from detector particles to the paper.

The anti-VEGF antibody that was being conjugated to the 15 nanometer gold was then used for the conjugate pad. This solution was spotted onto a piece of 3 millimeter by 1 millimeter fiberglass membrane using a pipette, until the pad was entirely saturated at around $8 \mu 1$. It was then allowed to dry in a six-well plate for an hour at room temperature. 
The conjugate pad and a fiberglass absorbent pad were placed down onto the designed nitrocellulose chip. Tape was placed on top of the entire chip with the exception of the area containing the sample input. This created a positive pressure to allow easier bridging between the paper and fiberglass pads for the VEGF sample. Then, a cut piece of polyethylene transparency (from Staples) was placed on top of the exposed doublesided tape.

$20 \mu \mathrm{L}$ of $0.17 \mu \mathrm{g} / \mathrm{ul}$ recombinant human VEGF 165 was pipetted into the input of the chip. Then the test was allowed to run and signified its finish as the absorbent pad began to soak up the excess sample in the test. A table demonstrating the concentrations and volumes associated with this test may be found in Table 1.

\section{Table I: Conjugating Gold to Antibody Concentrations}

Volumes and Concentrations of Antibodies and VEGF Used in the development of a twodimensional lateral flow assay where gold was conjugated to the detection particle.

\begin{tabular}{|c|c|c|c|c|c|c|}
\hline Antibody & $\begin{array}{c}\text { Volume } \\
\text { of } \\
\text { Antibody }\end{array}$ & $\begin{array}{c}\text { Concentration } \\
\text { of Antibody }\end{array}$ & $\begin{array}{c}\text { VEGF } \\
\text { Volume }\end{array}$ & $\begin{array}{c}\text { VEGF } \\
\text { Concentration }\end{array}$ & $\begin{array}{c}\text { Gold } \\
\text { Volume }\end{array}$ & $\begin{array}{c}\text { Gold } \\
\text { Concentration }\end{array}$ \\
\hline $\mathrm{IgG}$ & $1 \mu \mathrm{L}$ & $1 \mathrm{mg} / \mathrm{ml}$ & $20 \mu \mathrm{L}$ & $0.17 \mu \mathrm{g} / \mathrm{ul}$ & $8 \mu \mathrm{L}$ & $20 \mu \mathrm{g} / \mathrm{ml}$ \\
\hline $\mathrm{VEGF}$ & $1 \mu \mathrm{L}$ & $1 \mathrm{mg} / \mathrm{ml}$ & $20 \mu \mathrm{L}$ & $0.17 \mu \mathrm{g} / \mathrm{ul}$ & $8 \mu \mathrm{L}$ & $20 \mu \mathrm{g} / \mathrm{ml}$ \\
\hline
\end{tabular}

\section{Monoclonal Antibody Detection}

Following the gold-antibody conjugation experiments, additional monoclonal anti-VEGF antibodies were purchased that previously were conjugated to 30 nanometer gold particles. 30 to 50 nanometer gold particles sizes are frequently used in lateral flow assays [47-49]. This detector particle was purchased from BIOSS-USA. The antibody was conjugated to gold upon purchase in its fragment crystallizable $(\mathrm{Fc})$ region. Its immunogen sequence is:

PQTCKCSCKNT 158-168/191 [50] 
The letter sequence provided is representative of the amino acids between numbers 158 and 168 of a 191 total letter sequence. This means as the antibody is exposed to the VEGF signal protein, it will attach to a particular area of the protein, and no longer allow for any other antibodies to bind to this region. This provided immunogen sequence was run to see its specificity of attachment to different proteins using a Basic Local Alignment Search Tool (BLAST) [50, 51]. A BLAST finds regions of similarity between biological sequences, whether it is through analyzing a nucleotide or protein sequence. Using a protein BLAST, the given sequence was entered, and run through a database, which produced proteins with sequences that had significant and less than significant alignment with the particular antibody or protein of interest. Significance is defined as the closer the protein's E-value (Expect-value) is to zero [51]. An E-value is defined as, "a parameter that describes the number of hits one can "expect" to see by chance when searching a database of a particular size. It decreases exponentially as the Score $(S)$ of the match increases. Essentially, the E value describes the random background noise. For example, an E value of 1 assigned to a hit can be interpreted as meaning that in a database of the current size one might expect to see 1 match with a similar score simply by chance" [51]. When a BLAST was run, one significant match was human VEGF VE5 $_{165}$ isoform, which was the particle that the experiment was designed to detect. Therefore, it was appropriate to proceed using this antibody as a detector particle in the experiment. Further step-by-step instructions on the usage of BLAST may be found in Appendix E. The initial antibody that was used as a capture reagent was identical to the VEGF antibody purchased from BIOSS-USA, but without conjugated gold nanoparticles. 
To place antibodies at the detection line, a spotting technique (known as a spot blot) was used. Initially, the lyophilized VEGF antibodies were reconstituted using $1 \mathrm{X}$ PBS. Their concentrations, for both gold conjugated and no gold, were kept at $1 \mathrm{mg} / \mathrm{ml}$. However, according to literature the gold conjugated antibody was also diluted to a concentration of $10 \mu \mathrm{g} / \mathrm{ml}$ to test as well [52]. Dilution calculations for these antibodies may be found in Appendix D. A fine tip pipette was filled with $2 \mu \mathrm{L}$ of the nonconjugated VEGF antibody and a line was slowly drawn across the membrane in its appropriately designed well in for its application. Additionally, $2 \mu \mathrm{L}$ of IgG was deposited in a detection well (see instructions for using $\mathrm{IgG}$ in the Conjugating Gold to Antibodies section in the Methods). These were dried at both room temperature and in an oven at $37^{\circ} \mathrm{C}$ for two hours. Also, of these, half were incubated in $1 \%$ BSA (fully submerged) for 7 minutes and washed in $0.05 \%$ SDS and $2 \mathrm{mM} \mathrm{Na}_{2} \mathrm{HPO}_{4}$ solution for 10 minutes.

In this experiment, several designs were produced using AutoCAD. Unlike the design that was developed for conjugating gold to antibodies, this design had two wells instead of one and the detection regions were made much larger to help aid in visualization by the user for detection. The purpose of the two wells was to allow for further separation the $\mathrm{IgG}$ and anti-VEGF detection antibodies on the membrane. Two different channel widths (2.4 millimeters and 1.2 millimeters) were tested in order to see if this made any significant differences in detection signal from the gold. These two designs may be seen in Figure 16 and Figure 17. 


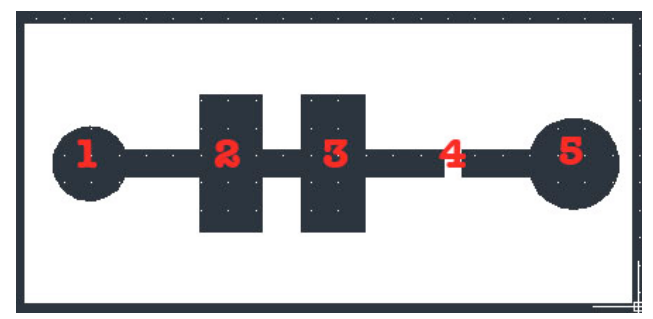

Figure 16: Large Square Well Biochemistry Chip. 2D chip used for detecting VEGF with 30 nanometer gold particle and a channel width of 2.4 millimeters. (1) Absorbent region; (2) control region; (3) the detection region; (4) wax barrier; (5) the sample input region.

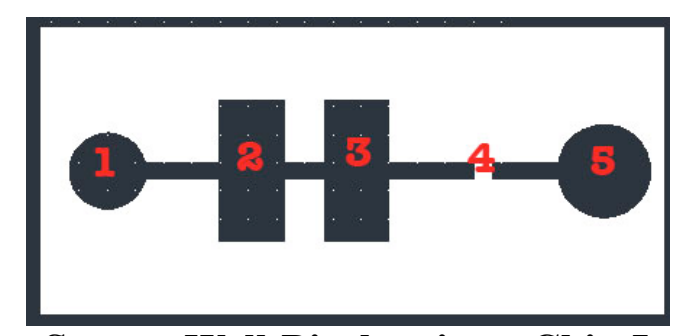

Figure 17: Large Square Well Biochemistry Chip Iteration 2. 2D chip used for detecting VEGF with 30 nanometer gold particle and a channel width of 1.2 millimeters. (1) Absorbent region; (2) control region; (3) the detection region; (4) wax barrier; (5) the sample input region.

Commonly wicking of the capture reagents would take place to a point that the antibodies in one well would travel to another detection well of the design. This could add to erroneous results, so a third iteration of the design was developed in order to help limit the travel of liquid from one area to another. Wax barriers were printed between the wells so that when antibodies were placed down on the nitrocellulose surface, they could not wick. From there, a set of "jumpers" were developed, which involved small hand cut pieces of unbacked nitrocellulose membrane. Therefore, as a VEGF sample would travel from the input region down the 2.4 millimeter channel, it would reach the nitrocellulose jumper and wick onto it and through it, into the detection wells. The ability for the VEGF sample to transfer from the base membrane to the jumper and back into the detection well was aided by positive pressure provided by double-sided tape that was laid on top of and 
pressed down upon the chip's apparatus, and covered with an optically clear transparency for viewing purposes. The AutoCAD design of this concept may be found in Figure 18.

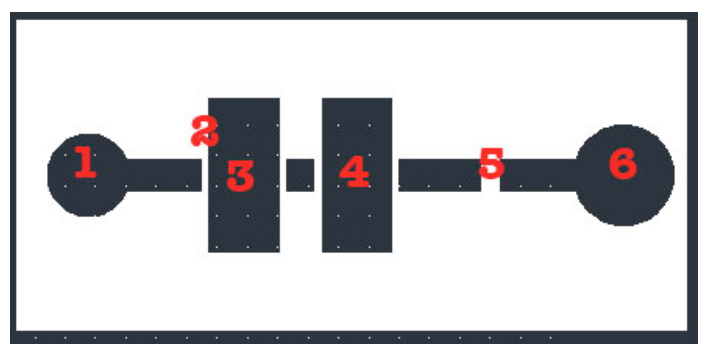

Figure 18: Jumper Design Biochemistry Chip. 2D chip used for detecting VEGF in sample where with 30 nanometer gold particle and a channel width of 2.4 millimeters. (1) Absorbent region; (2) jumper wax barrier; (3) control region; (4) detection region; (5) wax barrier for conjugate pad; (6) the sample input region.

Additionally, with the procedure stated above for the monoclonal antibodies, different combinations of the washes and post-treatments, as well as time taken for those procedures were varied in order to see if there was an optimal combination to yield the strongest signal for detection of VEGF. These combinations of each test may be found in Table II (for anti-VEGF) and Table III (for IgG). To note, many of these tests show repeated post-treatments and washes of the membrane, however these tests also incorporated other combinations of treatments with the information provided in Table II and III, which will be explained further. 
Table II: Post-treatment and Washes for Monoclonal anti-VEGF Antibody

Capture antibody anti-VEGF and the respective post-treatments and washes that were tested in order to find the optimal combination that yielded the strongest signal for VEGF detection.

\begin{tabular}{|c|c|c|}
\hline \multicolumn{3}{|c|}{ Capture Antibody: Anti-VEGF } \\
\hline Test & Post-Treatment & Wash \\
\hline 1 & None & None \\
\hline 2 & $1 \%$ BSA for 10 minutes & $\begin{array}{l}0.05 \% \mathrm{SDS} / 5 \mathrm{mM} \\
\mathrm{Na}_{2} \mathrm{HPO}_{4} \text { for } 7 \mathrm{~min}\end{array}$ \\
\hline 3 & None & None \\
\hline 4 & None & None \\
\hline 5 & $1 \%$ BSA for 12 minutes & $\begin{array}{l}0.05 \% \mathrm{SDS} / 5 \mathrm{mM} \\
\mathrm{Na}_{2} \mathrm{HPO}_{4} \text { for } 11 \mathrm{~min}\end{array}$ \\
\hline 6 & $1 \%$ BSA for 10 minutes & $\begin{array}{l}0.05 \% \mathrm{SDS}^{2} 5 \mathrm{mM} \\
\mathrm{Na}_{2} \mathrm{HPO}_{4} \text { for } 7 \mathrm{~min}\end{array}$ \\
\hline 7 & $1 \%$ BSA for 10 minutes & $\begin{array}{l}0.05 \% \mathrm{SDS}^{2} 5 \mathrm{mM} \\
\mathrm{Na}_{2} \mathrm{HPO}_{4} \text { for } 7 \mathrm{~min}\end{array}$ \\
\hline 8 & $1 \%$ BSA for 11 minutes & $\begin{array}{l}0.05 \% \mathrm{SDS} / 5 \mathrm{mM} \\
\mathrm{Na}_{2} \mathrm{HPO}_{4} \text { for } 11 \mathrm{~min}\end{array}$ \\
\hline 9 & $1 \%$ BSA for 11 minutes & $\begin{array}{l}0.05 \% \mathrm{SDS}^{2} 5 \mathrm{mM} \\
\mathrm{Na}_{2} \mathrm{HPO}_{4} \text { for } 11 \mathrm{~min}\end{array}$ \\
\hline 10 & $1 \%$ BSA for 10 minutes & $\begin{array}{l}0.05 \% \mathrm{SDS}^{2} 5 \mathrm{mM} \\
\mathrm{Na}_{2} \mathrm{HPO}_{4} \text { for } 11 \mathrm{~min}\end{array}$ \\
\hline 11 & $1 \%$ BSA for 10 minutes & $\begin{array}{l}0.05 \% \mathrm{SDS} / 5 \mathrm{mM} \\
\mathrm{Na}_{2} \mathrm{HPO}_{4} \text { for } 11 \mathrm{~min}\end{array}$ \\
\hline
\end{tabular}

Table III: Post-Treatment and Washes for IgG Antibody

Capture antibody IgG and the respective post-treatments and washes that were tested in order to find the optimal combination that yielded the strongest signal for VEGF detection

\begin{tabular}{|c|c|c|}
\hline \multicolumn{3}{|c|}{ Capture Antibody: IgG } \\
\hline Test & Post-Treatment & Wash \\
\hline 1 & None & None \\
\hline 2 & $1 \%$ BSA for 10 minutes & $\begin{array}{l}0.05 \% \mathrm{SDS} / 5 \mathrm{mM} \\
\mathrm{Na}_{2} \mathrm{HPO}_{4} \text { for } 7 \mathrm{~min}\end{array}$ \\
\hline 3 & None & None \\
\hline 4 & None & None \\
\hline 5 & $1 \%$ BSA for 12 minutes & $\begin{array}{l}0.05 \% \mathrm{SDS} / 5 \mathrm{mM} \\
\mathrm{Na}_{2} \mathrm{HPO}_{4} \text { for } 11 \mathrm{~min}\end{array}$ \\
\hline 6 & $1 \%$ BSA for 10 minutes & $\begin{array}{l}0.05 \% \mathrm{SDS} / 5 \mathrm{mM} \\
\mathrm{Na}_{2} \mathrm{HPO}_{4} \text { for } 7 \mathrm{~min}\end{array}$ \\
\hline 7 & $1 \%$ BSA for 10 minutes & $\begin{array}{l}0.05 \% \mathrm{SDS} / 5 \mathrm{mM} \\
\mathrm{Na}_{2} \mathrm{HPO}_{4} \text { for } 7 \mathrm{~min}\end{array}$ \\
\hline
\end{tabular}


Other things that were varied included if the test was a lateral flow assay, or purely a spot test. A spot test was where the capture antibody was immobilized to the nitrocellulose membrane, the VEGF sample was spotted on top of it, and then the gold conjugated antibody was spotted on top of that. This was tested in compare signal strength to that of a lateral flow assay. A spot test was hypothesized to show a stronger visual signal, as reagent would not be lost through the channels of the device.

Additionally, in some scenarios a conjugate pad containing gold colloidal particle with the antibody was used, and in other cases that detector particle was combined with VEGF and together was inputted into the test and run - in which case there was also a slight design alteration for the chip itself. In the lateral scenario, there was no conjugate pad used. This was in order to see if the conjugate pad aided in or hindered signal strength in detection.

Likewise, drying the membrane and/or the conjugate pad via an oven for two hours at $37^{\circ} \mathrm{C}$ or room temperature for one to two hours was also done. In literature, both have been used and aided in providing explanation for the results. A representation of these combinations and tests that were mentioned can be found in Appendix F.

\section{Polyclonal Antibody Detection}

The next anti-VEGF antibody used as a capture reagent was a polyclonal antiVEGF antibody. In general, most epitopes have not been mapped that are validated for use in ELISA or sandwich ELISA assays using human sample (in this case human VEGF $_{165}$ isoform). Because of this, it was important to use an antibody that was raised against the full length of the VEGF protein. This was to ensure that it would be able to detect epitopes that are not blocked by the detection antibody, explained previously. It 
was discovered that when the same immunogen sequence was of use for both antibodies, this would create a competitive attachment between the antibodies in the detection and capture regions, and no signal would be found in the test. Therefore, this full sequence polyclonal anti-VEGF antibody from Abcam was used for the next set of experiments.

The capture region for the detection line of the experiment was designed as a sandwich ELISA. This means, as explained in the Introduction, the capture antibody (polyclonal anti-VEGF) is tethered down to the nitrocellulose paper, while the inputted VEGF sample and anti-VEGF antibody run through the test. As they reach the detection region, the tethered antibody attaches to one region of the VEGF and the detector antibody conjugated to gold attaches to a separate, and specified, region of the VEGF sample. This entire apparatus looks like a "sandwich" as the sample is wedged between two antibodies.

A control line was no longer viable at this point of the experiment. As with before, the previous IgG that was purchased was a rabbit anti-mouse antibody. Due to the change in purchased antibodies, the only available conjugated gold anti-VEGF antibody in this experiment was an anti-rabbit formulation. This confliction removes the ability of the antibodies to bind to one another because they would either have to be rabbit and antirabbit, or mouse and anti-mouse. Therefore, this set of experiments removed the ability for use of a control line.

As previously, the gold conjugated anti-VEGF antibody was diluted to a concentration of $1 \mathrm{mg} / \mathrm{ml}$. This time when being diluted, distilled water was used rather than phosphate buffered saline (either can be used). Prior to the addition of the distilled 
water, its $\mathrm{pH}$ was checked to be around 7.4. When the $\mathrm{pH}$ was not around this range, buffers were added and $\mathrm{pH}$ strips were used in order to ensure correct a correct level.

Instead of spot blotting the designed nitrocellulose chip in free-form, designs were implemented to spot a unique pattern onto the chip's surface. A design was laser cut into a standard transparency, made from cellulose acetate. This transparency design was used as a grid that aided in maintaining a consistent application of antibody onto the twodimensional nitrocellulose chip. An " $\mathrm{X}$ " shape design was implemented using AutoCAD, for the area in which the capture reagent would be applied on the chip (this can be found in Figure 19). This design was chosen alternatively to a dot or a line in order to allow for visual detection of a distinct shape. The settings for the laser cutter were set to mylar with a 0.05 inch depth for cutting. The end result of the laser cut transparency may be found in Figure 20. With these "X" shaped designs, more two-dimensional nitrocellulose chips were developed in AutoCAD. Unlike with previous designs, the detection regions were rounded as opposed to square in order to limit dead area for the colloidal gold or VEGF sample to congregate. Two designs were developed in order to see if there were any differences in detection signal strength as opposed to size of the chip and channel distance. These designs are further described in Figures 21.

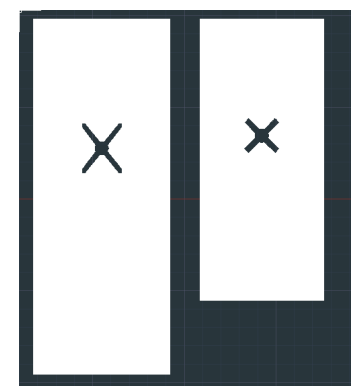

Figure 19: X-Shaped Transparency. Design of " $X$ " shaped transparency to use as a striper pattern in order to lay down capture reagents onto the $2 \mathrm{D}$ paper microfluidic chip. 


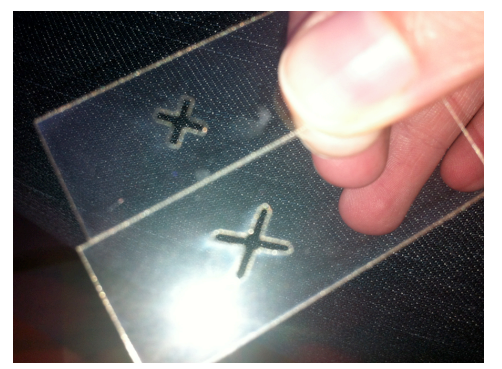

Figure 20: Laser Cut X-Shaped Transparency. " $X$ " shaped transparency made of cellulose acetate post-laser cutting to use as a striper pattern for capture reagents.

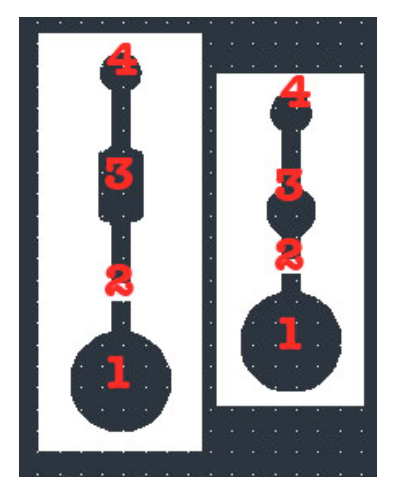

Figure 21: Labeled Circular 2D Chips for Biochemistry. 2D chips used for detecting VEGF in sample where with 30 nanometer gold particle and a channel width of 2.4 millimeters. (1) Sample input region; (2) wax barrier; (3) the detection region; (4) the absorbent region.

This design was laid over the top of the nitrocellulose chip and $3 \mu \mathrm{L}$ of the capture antibody solution was spotted onto the paper, following the X-shaped pattern. The spotted solution was dried by placing the nitrocellulose chips in the oven for two hours at $37^{\circ} \mathrm{C}$. The chip was removed from the oven and entirely submerged in $1 \% \mathrm{BSA}$ for 7 minutes time. It was then "washed" - i.e. completely submerged - in a $0.05 \%$ SDS and $0.5 \mathrm{M} \mathrm{Na}_{2} \mathrm{HPO}_{4}$ for 10 minutes. The chips were then dried at room temperature for an hour.

In the meantime, a conjugate pad was prepared to place the anti-VEGF antibodies with gold on. The fiberglass pads were cut to a size of 5 millimeters by 2 millimeters. 
They were pretreated by being dipped in a solution of $1 \%$ sucrose and $2 \mathrm{mM}$ borate solution and allowed to dry at room temperature for about an hour's time. The conjugate pads were then submerged in the diluted gold-antibody solution and dried on a nonwicking surface (i.e. a 6-well plate made from polycarbonate) at $37^{\circ} \mathrm{C}$ for two hours. Additionally, $20 \mu \mathrm{L}$ of VEGF 165 signal protein and $10 \mu \mathrm{L}$ were added together into a conical tube, vortexed for 30 seconds, and allowed to incubate at room temperature for 20 minutes.

The chips were assembled by placing down the prepared conjugate pad down on the chip and the cellulose Whatman absorbent pad at their appropriate locations and taping them down onto the nitrocellulose chip. This promotes positive pressure to allow for a better bridge that the VEGF sample can bridge between the nitrocellulose and conjugate pad. Then, a cut-to-size transparency is placed on top of the tape in order to allow the experiment to be viewed, but to remove the double-sided tape from sticking to any other surfaces.

Three tests were conducted. The initial test served as a control. A sample of pure PBS with no trace of VEGF was inputted into the chip and allowed to run through the conjugate pad and to the detection region. If a band of colloidal gold could be seen, then the test would have a false positive, and if there was no band, that means that the test would have run appropriately to show no VEGF was detected. The second test that was run was the incubated sample of VEGF and anti-VEGF gold conjugated antibody. The entire $120 \mu \mathrm{L}$ solution was inputted into the chip and the test was allowed to run and see if there was detection of VEGF. If the band appeared, this would mean that the reagent was detected, and if not that means an error was made in the procedure (see the Results 
section for possibilities of error). Additionally, the solution was commonly followed by inputting PBS in order to push the sample entirely through the test - known as a pulse chase assay. The design for the first and second tests are somewhat altered from the design seen in Figure 20, and can be found in Appendix C. The third experiment involved pipetting a sample of VEGF into the chip and having it wick through the treated conjugate pad, and to run through the test. This addition of the conjugate pad would allow for the paper microfluidic chip to closely follow a lateral flow assay found on the market. However, if it did not work it would allow for further investigation on the improvement for a conjugate pad containing gold and antibodies, as many errors arise with this portion of the test.

Further calculations were completed in order to determine optimal concentrations to use in this experiment. Initially, an experiment was conducted in order to determine the lowest concentration of gold conjugated antibody that would allow for optimal visualization by naked eye. A design was created in AutoCAD that had six wells (as a circular shape) that were two-millimeters in diameter. Six concentrations were prepared $(1 \mathrm{mg} / \mathrm{ml}, 0.5 \mathrm{mg} / \mathrm{ml}, 0.1 \mathrm{mg} / \mathrm{ml}, 0.05 \mathrm{mg} / \mathrm{ml}, 0.01 \mathrm{mg} / \mathrm{ml}$, and $0.002 \mathrm{mg} / \mathrm{ml})$ and 3 microliters from each were pipetted and deposited into their corresponding well. The wells were analyzed for the opacity of color created from the gold, and the lowest concentration that provided noticeable color was chosen. From there, that concentration along with the molecular weight of the antibody $\left(4.50 \times 10^{-4} \mathrm{grams} / \mathrm{mole}\right)$ was used in order to determine the molar concentration of the gold conjugated antibody (this is demonstrated in Equation 4). MW represents molecular weight, $\mathrm{c}_{\mathrm{i}}$ is the molar concentration, and $\rho_{\mathrm{i}}$ is the mass concentration. 


$$
c_{i}(\text { moles } / m l)=\frac{\rho_{i}(g / m l)}{M W(g / m o l e)}
$$

Finally, using the molar concentration and Avogadro's number $\left(6.022 \times 10^{23}\right.$ molecules $)$ the amount of molecules per milliliter of solution was determined for the gold conjugated antibody. $\mathrm{C}_{\mathrm{i}}$ represents the number concentration.

$$
C_{i}(\text { molecules } / m l)=\left[c_{i}(\text { moles } / m l)\right]\left[N_{A}\right]
$$

In theory, every antibody can bind to two separate antigens. Additionally, human VEGF $_{165}$ isoform is smaller in size than anti-VEGF monoclonal or polyclonal antibody. Therefore, theoretically, if there is a major excess of $\mathrm{VEGF}_{165}$ in solution, it can bind to the two sites on the gold conjugated monoclonal anti-VEGF antibody and, because it's smaller, can wick down the chip at a faster rate than the gold antibodies and bind to the monoclonal anti-VEGF antibody. If these proteins bound to all open sites of the antibodies, this means that it would not allow for antibodies to create a sandwich ELISA assay because all binding sites would be filled before the detector reagent reached the location of the capture reagent antibody. Therefore, there needed to be an even amount of VEGF $_{165}$ molecules to polyclonal anti-VEGF antibody (capture reagent) in the test, however it was allowable to have an excess of gold conjugated antibodies in the solution. So, a concentration below the optimal visual gold concentration was chosen to analyze for molecular concentration. This value was applied to analyzing VEGF and polyclonal anti-VEGF concentrations. One important thing to note is that $\mathrm{VEGF}_{165}$ needs to have a molecular concentration twice as large as that of the polyclonal anti-VEGF antibody, because the antibodies have two binding sites. The table providing the values of these calculations (all originating from Equations 1 and 2) can be found in Table IV.

\section{Table IV: Initial Concentration Calculation for VEGF Antibodies}


Calculations for appropriate concentrations of capture reagents, detection reagents, and VEGF sample for antibody-based lateral flow assay

\begin{tabular}{|c|c|}
\hline \multicolumn{2}{|c|}{ Concentration Calculations for VEGF Lateral Flow Assay } \\
\hline True Colloidal Gold Concentration + Anti-VEGF Antibody Concentration \\
\hline Concentration & $0.75 \mathrm{mg} / \mathrm{ml}$ \\
\hline Molecular Weight & $4.50 \times 10^{4} \mathrm{~g} / \mathrm{mole}$ \\
\hline Molar Concentration & $1.67 \times 10^{-8} \mathrm{moles} / \mathrm{ml}$ \\
\hline Molecular Concentration & $1.00 \times 10^{16} \mathrm{molecules} / \mathrm{ml}$ \\
\hline Colloidal Gold Concentration for Anti-VEGF Antibody and VEGF Concentrations \\
\hline Concentration & $0.50 \mathrm{mg} / \mathrm{ml}$ \\
\hline Molecular Weight & $4.50 \times 10^{4} \mathrm{~g} / \mathrm{mole}$ \\
\hline Molar Concentration & $1.11 \times 10^{-8} \mathrm{moles} / \mathrm{ml}$ \\
\hline Molecular Concentration & $6.69 \times 10^{15} \mathrm{molecules} / \mathrm{ml}$ \\
\hline \multicolumn{2}{|c|}{ VEGF Concentration } \\
\hline Concentration & $4.27 \times 10^{-4} \mathrm{~g} / \mathrm{mole}$ \\
\hline Molecular Weight & $19200 \mathrm{~g} / \mathrm{mole}$ \\
\hline Molar Concentration & $2.22 \times 10^{-8} \mathrm{moles} / \mathrm{ml}$ \\
\hline Molecular Concentration & $1.34 \times 10^{16} \mathrm{molecules} / \mathrm{ml}$ \\
\hline Polyclonal Anti-VEGF Concentration \\
\hline Concentration & $4.27 \times 10^{-4} \mathrm{~g} / \mathrm{mole}$ \\
\hline Molecular Weight & $38200 \mathrm{~g} / \mathrm{mole}$ \\
\hline Molar Concentration & $1.11 \times 10^{-8} \mathrm{moles} / \mathrm{ml}$ \\
\hline Molecular Concentration & $6.69 \times 10^{15} \mathrm{molecules} / \mathrm{ml}$ \\
\hline
\end{tabular}

The three experiments mentioned previously were repeated, using these calculated concentrations.

An alternative way to attach and test antibodies used a much different protocol than previous methods. This procedure was applied to both the previous concentrations as well as the calculated concentrations seen in Table $I V$. In this procedure, $3 \mu \mathrm{L}$ of the VEGF antibody was spotted down onto the detection region of the nitrocellulose membrane in an X pattern. The membrane was allowed to dry, covered, at room temperature for one hour. The membrane was then blocked in $1 \% \mathrm{w} / \mathrm{v}$ BSA by immersion for thirty minutes time. The platform was then rinsed once with water and allowed to fully dry at room temperature (taking around an hour). Then, the membrane 
was dipped in 5\% w/v sucrose solution and dried, to allow for the gold colloidal particles attached to antibodies to move easily down the membrane. The gold conjugated antibodies were spotted directly onto the membrane, near where the sample input was located on the test platform. This was allowed to dry for around an hour. $120 \mu \mathrm{L}$ of VEGF sample was inputted and the test was allowed to run. The test was declared finished as the sample reached the absorbent pad at the test's end.

All procedures mentioned previously that were used to test antibodies as capture reagents with the calculated concentrations, were repeated, but this time involving the aspect of humidity. As the nitrocellulose membranes were set to dry in either an oven at $37^{\circ} \mathrm{C}$ or room temperature, they were enclosed in a chamber containing small aliquots of water. In many cases, lack of humidity causes evaporation of the antibody from the material surface [38]. Therefore, in order to aid in antibody binding to the surface of the nitrocellulose, this step was implemented into the procedure.

Further calculations regarding antibody and VEGF concentrations were done. These calculations were important for recognizing the amount of gold that could be seen by the human eye, considering number of gold colloidal particles, visible depth of the lateral flow assay, and sample spot volumes. Initially the molecular weight of colloidal gold, mass concentration of gold conjugated antibodies, density of gold, and radius of the gold particle were given values. The volume of the gold particles was calculated and from there, and from considering liquid loss throughout the chip's length and depth, the amount of molecules present at detection was determined. However, the depth of which one can see gold signal was also taken into account using Equation 6 [23].

$$
\% \text { VisibleSignal }=\frac{\text { VisibleDepth }(\sim 10 \mu m)}{\text { MembraneThickness }}
$$


Considering these values, the volume as well as concentrations of VEGF gold conjugated anti-VEGF antibody, and polyclonal anti-VEGF antibody were determined. Further calculations and steps may be found in Appendix G. The calculated concentrations and volumes for the VEGF, polyclonal capture antibody, and gold conjugated anti-VEGF antibody are located in Table $\mathrm{V}$.

Table V: Minimum Concentration Calculations

Calculated Concentrations and Volumes For Antibody Lateral Flow Assay Test

\begin{tabular}{|c|c|c|}
\hline Reagent & Volume & Concentration \\
\hline VEGF & $120 \mu \mathrm{L}$ & $6.40 \mathrm{ng} / \mathrm{ml}$ \\
\hline $\begin{array}{c}\text { Polyclonal Anti-VEGF } \\
\text { Antibody }\end{array}$ & $0.988 \mu \mathrm{L}$ & $0.25 \mu \mathrm{g} / \mathrm{ml}$ \\
\hline $\begin{array}{c}\text { Gold Conjugated Anti- } \\
\text { VEGF Antibody }\end{array}$ & $\begin{array}{c}\text { N/A - conjugate pad } \\
\text { saturation }\end{array}$ & $0.75 \mathrm{mg} / \mathrm{ml}$ \\
\hline
\end{tabular}

Note that these concentrations are theoretically the minimum amounts of each concentration and volume that would be needed in order to show visual signal. The volume for the gold conjugated antibody was not specified due to the fact that the material was dipped into the solution and therefore it could not be determined.

\section{Aptamer Testing}

For the aptamer, a concentration of $5 \mathrm{mM}$ was applied using $3 \mu \mathrm{L}$ of reconstituted oligos. In order to resuspend the aptamers, the tube must be briefly spun before opening. Then, mix the dried aptamer with a $\mathrm{pH}$ of between 7.0 and 8.0 in distilled water and vortex the solution. Distilled water tends to have a much lower (or more acidic) $\mathrm{pH}$, so a buffer with a pH of 8 was commonly added prior to exposing it to the aptamers. The solution was then equilibrated and after five minutes. The oligonucleotides were vortexed again in order to fully finish its resuspension. The reconstituted aptamers were further diluted to a concentration of $10 \mathrm{mg} / \mathrm{ml}$ using deionized water as a buffer solution. Using a narrow-mouth pipette tip, $1.5 \mu \mathrm{L}$ of the sample was spotted onto the nitrocellulose. This 
was spotted in the same manner as the antibodies were onto nitrocellulose, using a laser cut transparency with a unique design to distinguish signal as being positive rather than a false positive. The membrane was dried via baking in an oven for two hours at $80^{\circ} \mathrm{C}[52$, 53]. After drying, the membrane was blocked so that no non-specific binding could occur using $1 \%$ BSA for seven minutes room temperature. This was followed by a $0.05 \%$ SDS and $2 \mathrm{mM} \mathrm{Na}_{2} \mathrm{HPO}_{4}$ wash for ten minutes [23]. Then the chip was dried at room temperature.

As was done for the antibody experiments, three tests were set up. One experiment was a control, where a sample of PBS that did not contain any VEGF was inputted into a two-dimensional chip. The second experiment was combining VEGF sample and anti-VEGF antibodies conjugated to colloidal gold and inputting those into the test chip. The final test was using a conjugate pad loaded with gold conjugated antiVEGF antibody, and inputting a sample of VEGF to the chip, similar to a lateral flow assay, and seeing if sample could be detected. For further detail, refer to experimental description in Gold Conjugated to Antibodies in the Methods section.

In order to prove that the antibodies and aptamers were functional, a two dimensional chip was designed that had one channel and one conjugate pad with antiVEGF antibody conjugated with $30 \mathrm{~nm}$ colloidal gold. The membrane was spotted, as mentioned above, as well. A sample containing human VEGF was inputted into the chip and allowed to wick down the entirety of the chip to the detection region to observe possible detection of the signaling protein.

Additionally, biotinylated aptamers were purchased. Commonly it is difficult to detect visual signal from an aptamer that has less than 500 base pairs (in this case there 
are 28). Therefore, by adding a biotin to the 5' end of the custom Vap7 sequence, it will have a high affinity for binding to streptavidin [54]. The streptavidin was pipetted to the nitrocellulose membrane surface in the regions of detection. Per each detection region, 4 $\mu \mathrm{L}$ at a concentration of $1 \mathrm{mg} / \mathrm{ml}$ was spotted down onto the membrane and set to dry for around 45 minutes time (or until the nitrocellulose membrane was entirely dry).

Afterwards, the diluted biotinylated aptamer ( $4 \mu \mathrm{L}$ with a $1 \mathrm{mg} / \mathrm{ml}$ concentration) was again spotted down in the detection region and allowed to dry. Important to note is that as the chips were drying, they were placed in a chamber containing wells with water in order to maintain humidity in the chamber (like with the antibody procedure) in order to limit any evaporation from the surface of the membrane and to promote binding. The membrane was blocked using $1 \%$ BSA for seven minutes and followed by a $0.05 \%$ SDS and $2 \mathrm{mM} \mathrm{Na}_{2} \mathrm{HPO}_{4}$ wash for ten minutes [23]. The conjugate pad process followed the same procedure as previously used. VEGF sample at a concentration of $50 \mathrm{ng} / \mathrm{ml}$ was processed through the chip and the test was visually analyzed for detection. Rather than spotting a particular volume onto the chip, it was dipped into the VEGF solution and allowed to run for its duration. 


\section{Results}

Following the completion of experiments requiring numerical data, all points were organized into tables using Microsoft Excel and transferred to Minitab if statistical analysis was necessary. Statistical analyses used multi-way analysis of variance. Both observational and numerical results will be presented within this section. For further explanation of the results and its relationship with the overall objectives of this thesis project, this will be found in the Discussion section.

\section{Characterization}

The characterization portion of this thesis considered a multitude of factors that could contribute to the flow of sample throughout a nitrocellulose membrane and compared it to other materials as well as different pore sizes of the nitrocellulose itself.

\section{Channel Widths and Wax Reflow}

The line and circle designs with different thicknesses were developed in order to analyze limits of detection. However, after developing these channels, they were not analyzed in this project but were instead implemented into another thesis project regarding the limits of detection of wax channels in paper microfluidics. These channel designs may be found in Appendix B. However, master's student Ryan Silva carried on this project. The importance of such a project can be found in the Discussion.

In attempting to compare channel widths to that of Yao Lu et al., channel thicknesses of $225 \mu \mathrm{m}, 250 \mu \mathrm{m}, 275 \mu \mathrm{m}$ and $285 \mu \mathrm{m}$ were printed on standard 8.5" by 11 " printer paper and melted. However, from this print it was seen that these channels

were far too small and could barely be seen by the human eye. Post-melting the wax into the chips on an open-face hot plate, the wax completely reflowed into the smaller 
channels making them unable to be seen, and therefore unable to run any liquid sample through the channel whatsoever. These designs were additionally printed onto the 0.45 $\mu \mathrm{m}$ and the $285 \mu \mathrm{m}$ and $275 \mu \mathrm{m}$ channels were relatively visible, but the small pore size and surfactant of the material did not allow for wicking through the membrane whatsoever.

Two types of paper, bibulous paper, and $0.45 \mu \mathrm{m}$ pore size nitrocellulose paper was testing for wicking times. The bibulous paper and printer paper wicked within seconds to minutes. This observational step also proved that the larger the channel width was designed into the paper, the quicker the liquid wicked from start to finish. It was also observed that curved channels took a longer time to wick than straight channels. The 0.45 $\mu \mathrm{m}$ nitrocellulose platform did not wick whatsoever, after placing down a droplet of green dye into the input of the paper and waiting for an hour time. The end of this test may be seen in Figure 22.
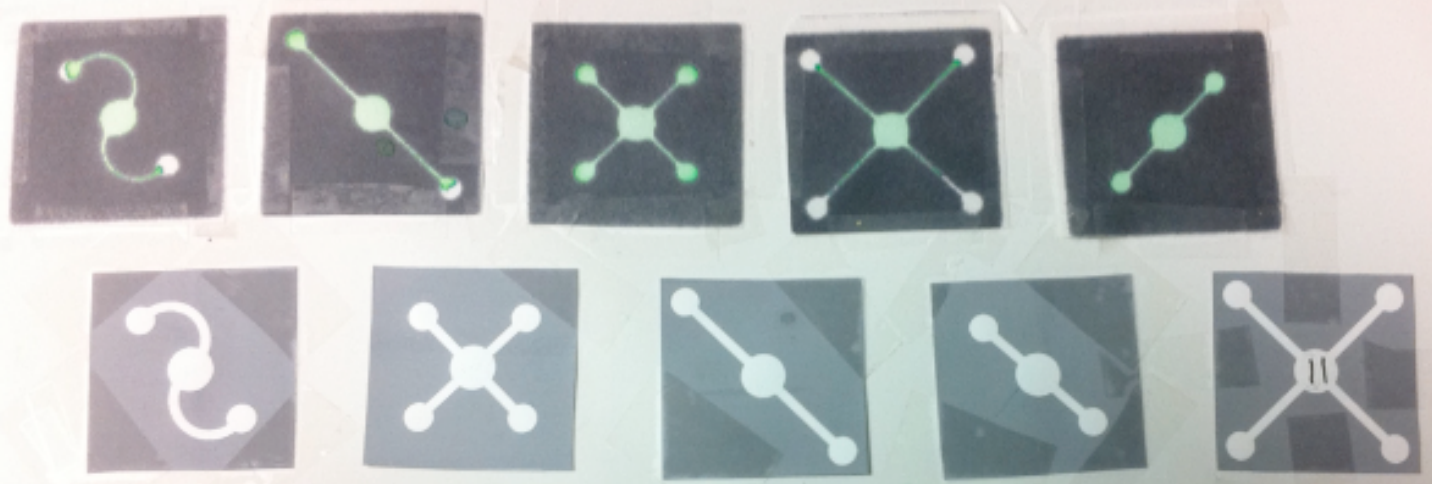

Figure 22: Bibulous and Nitrocellulose Comparison. Comparison of wicking for bibulous paper (top) versus $0.45 \mu \mathrm{m}$ pore size nitrocellulose (bottom) where green food coloring was used as a way to visualize the movement of sample through the channels. 


\section{Optimal Channel Characteristics}

A high flow 135 nitrocellulose membrane with a plastic backing adhered was analyzed for wicking time. Using three different channel widths, it was seen that the smallest channel width of 0.8 millimeters had the longest wicking time, to which some channels did not have completion of the liquid's passage from start to end. The fastest wicking time overall were for the 1.6-millimeter channel width designs, and the middle time was for that of the 2.4 millimeter channel widths. An image of the tests during their run may be found in Figure 23. Note how the smallest channel width (top) had two chips wicking at the same time that six of the seven middle channel width (middle) chips had completed their run.

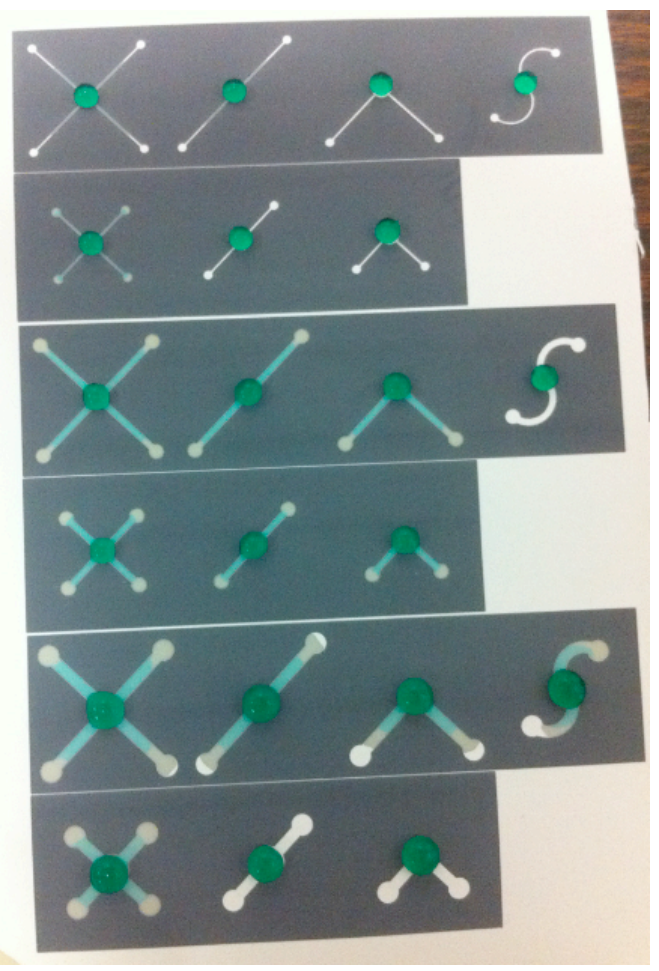

Figure 23: Channel Thickness Wicking Comparison. Backed HF 135 nitrocellulose membrane comprised of three channel widths $(0.8 \mathrm{~mm}, 1.6 \mathrm{~mm}$, and $2.4 \mathrm{~mm}$ from top to bottom, respectively) tested with green dye for wicking time differences for different channel widths, length and curvature. 
It was also noted that the curved patterns on the nitrocellulose wicked slower than the linear patterns, if at all. It can be seen from Figure 1 that the curved channels in the middle set of designs has no green dye along its channels - showing that it did not wick whatsoever. The concept of wicking time was tested in further detail and statistically measured for significance to provide further detail.

\section{Design Combinations and Wicking Times}

As stated in the Methods section, statistical analysis was completed that took into account the porosity of the paper, whether or not the membrane had a backing on it, curvature of the designs that were created, length of the channels, and the width of the channels. The initial model of the $2 \mathrm{D}$ chip multi-way analysis of variance (ANOVA) was done using a completely randomized design (CRD) with five factors. All of the factors, as mentioned earlier, were fixed categorical variables containing two levels each. The two sets of experiments that were done were randomized using Microsoft Excel. These tables for all combinations and their experimental order can be found in Appendix A.

This data was run through Minitab (which can be found in Appendix A) and a four-in-one box plot was generated. When analyzing ANOVA, for the statistical method to be viable, four assumptions must be maintained, normality, constant variance, independence, and model adequacy. Because these assumptions were violated, the data first run in its original form, and then transformed using a logarithm (base ten), which was applied to all data points. This transformation allowed for all assumptions to be maintained. The non-transformed four-in-one box plot can be found in Figure 24 and transformed box plot can be found in Figure 25. Further explanation of the assumptions can be found in the Discussion. The analysis of variance results yielded significance for 
characteristics of the platform that had significant interactions for Backing*Curving*Porosity*Width and Backing*Size*Width by using an overall significance level of $10 \%$ and an individual significance level of $0.323 \%$. The Minitab output of this interaction can be found in Appendix A. The significance of these interactions and how these results were concluded is further explained in the Discussion section. For the interactions between the backing of the paper, curvature of the channel design, the porosity of the paper, and the width of the designed channel, had a p-value of 0.001 (which is less than $0.323 \%$, or a p-value of 0.00323 ). The next interaction to be significant (p-value of 0.002) contained Backing*Size*Width. The Minitab output for this interaction may also be found in Appendix A.

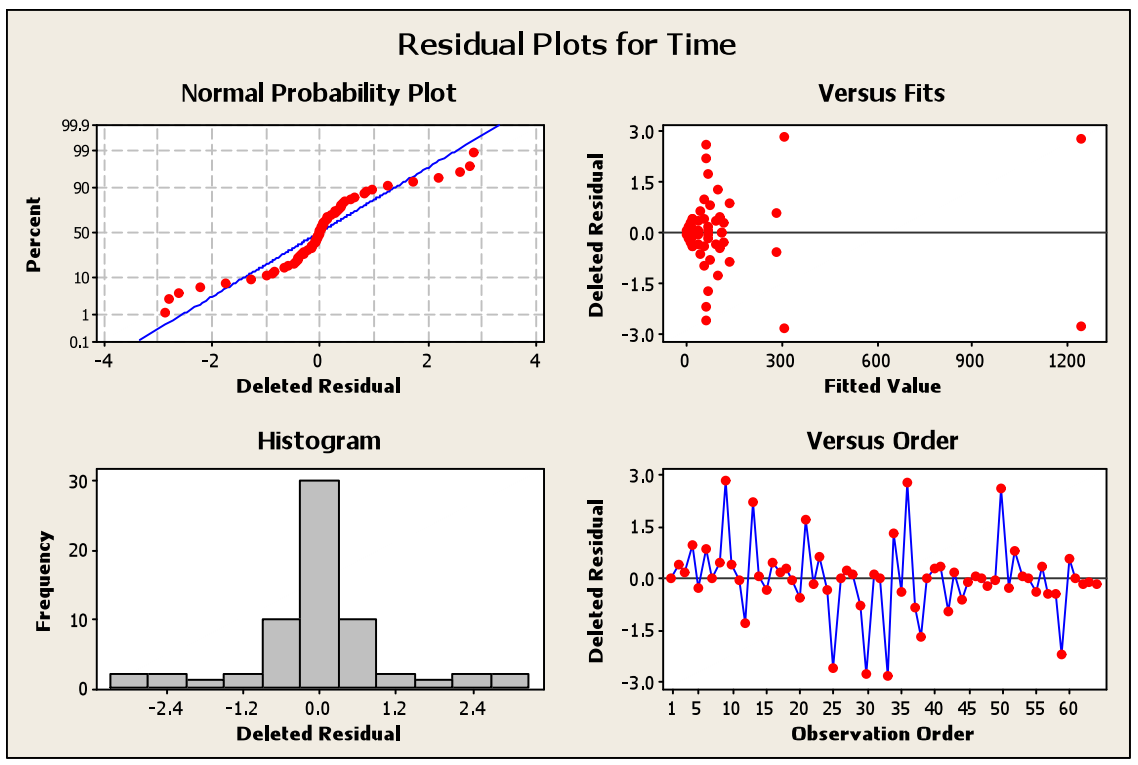

Figure 24: Untransformed Four-in-One Box Plot. Four-in-one box plot of original untransformed data that analyzes the backing of the membrane, porosity, curvature in the channels, channel width, and channel length. 


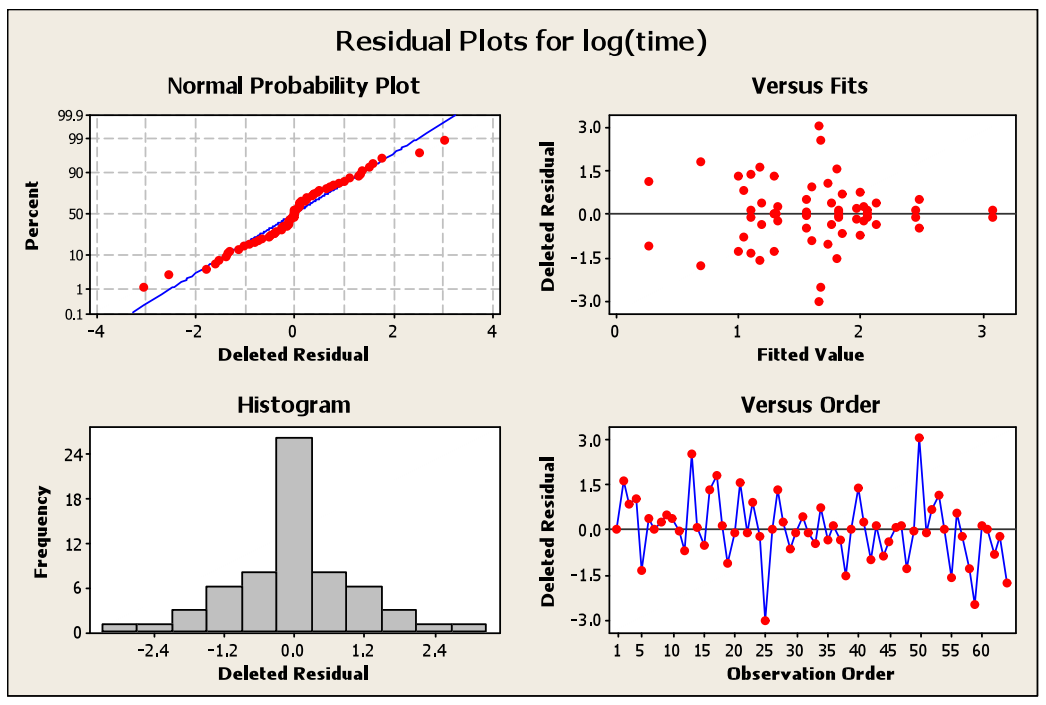

Figure 25: Transformed Four-in-One Box Plot. Four-in-one box plot of logarithmically transformed data in order to appropriately analyze the statistical data on wicking time without violating the assumptions of an ANOVA.

\section{Wicking Time - Conjugate Pad vs. Membrane Paper}

Because a large portion of this thesis was devoted to developing a three-

dimensional chip that allowed for simultaneous detection of target analytes from a single sample, it was important to characterize the materials used in order to see if this was possible (i.e. if there are changes in fiber orientation, if its an anisotropic material, et cetera). The two experiments were set up where four channels, one using a conjugate pad and one not using a conjugate pad, were compared to see the time difference between the channels in each experiment, and against one another (Table VI).

\section{Table VI: Wicking Comparison Times}

Times for testing four channels with and without an attached conjugate pad for overall wicking time

\begin{tabular}{|c|c|c|}
\hline Test & $\begin{array}{c}\text { With Conjugate Pad Time } \\
\text { (seconds) }\end{array}$ & $\begin{array}{c}\text { Without Conjugate Pad Time } \\
\text { (seconds) }\end{array}$ \\
\hline 1 & 207.8 & 41.1 \\
\hline 2 & 429.6 & 43.1 \\
\hline 3 & 392.1 & 42.6 \\
\hline 4 & 701.9 & 44.1 \\
\hline Standard deviation & 203.9 & 1.25 \\
\hline Average & 432.85 & 42.73 \\
\hline \multicolumn{2}{|c|}{ p-value: 0.0314} \\
\hline
\end{tabular}


The experiment yielded a standard deviation of 203.9 seconds and an average wicking time of 432.85 seconds for the experiment involving use of a conjugate pad, whereas the experiment without the conjugate pad had a standard deviation of 1.25 seconds and an average wicking time of 42.73 seconds. Comparing the two tests to one another using a t-test, this yielded a p-value of 0.0314 .

\section{Imaging}

Using the SEM, several nitrocellulose membranes were imaged that were both impregnated with wax and had none. In Figure 26, $0.45 \mu \mathrm{m}$ pore size nitrocellulose was imaged using a 3000X objective lens with no wax.

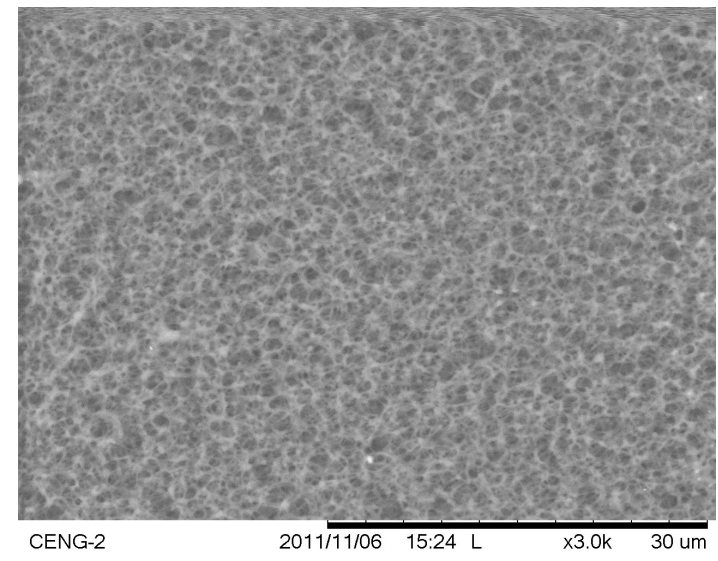

Figure 26: Smallest Nitrocellulose Pore Size SEM Image. A $0.45 \mu \mathrm{m}$ pore size piece of bare nitrocellulose membrane imaged using SEM at a 3000X objective lens.

Additionally, the HF 135 membrane was imaged in order to determine visual differences between a membrane that is impregnated with wax and one that is bare. Figure 27 demonstrates the membrane that has wax melted into its pores whereas Figure 28 shows the membrane as bare. 


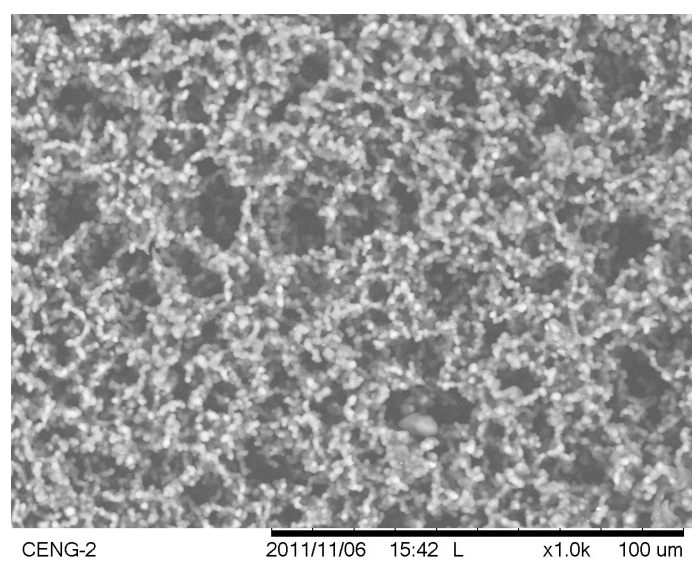

Figure 27: Wax Impregnated Membrane on SEM. HF 135 backed nitrocellulose membrane impregnated with wax in at 1000X magnification using the SEM.

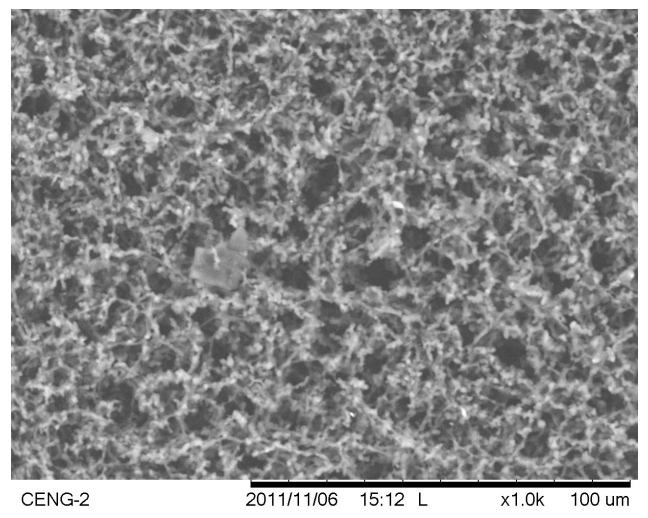

Figure 28: Bare Nitrocellulose Membrane on SEM. HF 135 backed bare nitrocellulose membrane at $1000 \mathrm{X}$ magnification using the SEM.

Likewise, it was of interest to determine if the SEM could detect the seam between where wax was melted into the pores of the paper and where the membrane was entirely bare. This was done at a 100X objective and may be found in Figure 29. 


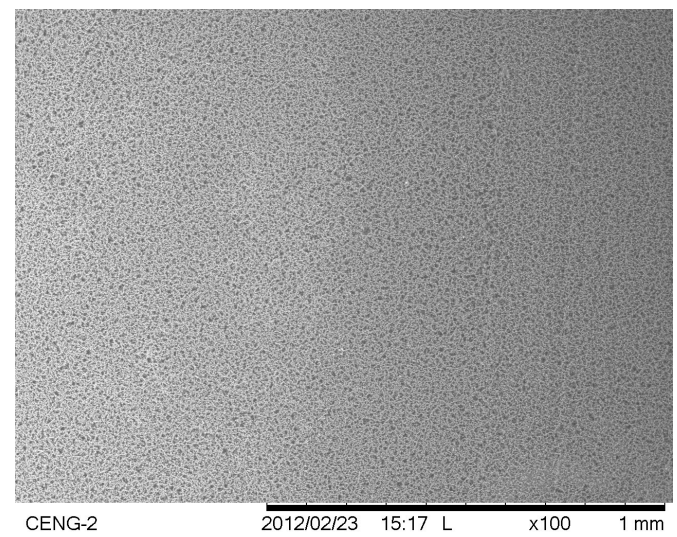

Figure 29: Seam Between Wax and Non-Wax on SEM. HF 135 backed nitrocellulose membrane at 100X objective lens showing the seam between wax (on right) and none (on the left) on the membrane's surface.

\section{Development of a Three-Dimensional Chip}

A multitude of components were analyzed in order to propose a feasible design for the simultaneous detection of four analytes in one single sample.

\section{Bridging Layer}

Three bridging techniques between paper layers were demonstrated in this thesis. The initial bridging technique was a powder that was created by using the $0.45 \mu \mathrm{m}$ pore size nitrocellulose saturated with acetone and grinding it with a mortar and pestal. However, after long amounts of hand grinding, the membrane failed to produce much, if any powdered substance of use.

The second powder technique instead used HF 180 or HF 135 unbacked nitrocellulose membrane in combination with acetone, grinding the mixture, and drying it on bibulous paper for later use. The powder was somewhat difficult to control in the assembly of the three-dimensional chips. Figure 30 demonstrates the difficulty behind controlling the limited area in which the powder was supposed to remain. 


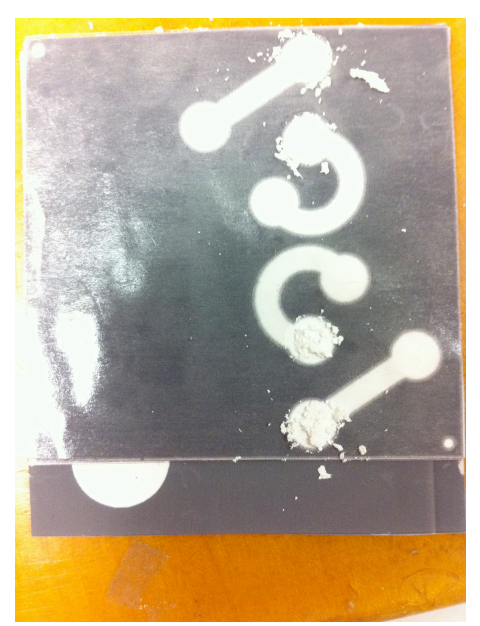

Figure 30: Powder Bridging Set-Up. The spread of nitrocellulose powder from its designated area during the assembly of the three-dimensional chip.

Additionally, the acetone and nitrocellulose yielded a wide range of powder particle sizes and shapes and each particle was relatively hard and brittle.

The third powder, developed from mixing water with nitrocellulose, was ground and shaped into the areas of bridging during the assembly of the chip. The grinding took significantly longer than that of using acetone and still needed to remain damp for use in the assembly. Also, powder sizes were still a wide range of sizes, but soft and ductile. The fourth powder was ground into a much finer powder size than the previous three, was just as soft of a powder as that created using water and nitrocellulose at room temperature, and was much more uniform in size compared to all other powder making techniques.

The lacquer, made from a 99\% acetone and nitrocellulose membrane, yielded a gelatinous material that was wiped across the membrane's surface. The lacquer material itself can be seen in Figure 31. When applying the lacquer to the surface of the nitrocellulose membrane, the nitrocellulose disintegrated, which is demonstrated in Figure 32. 


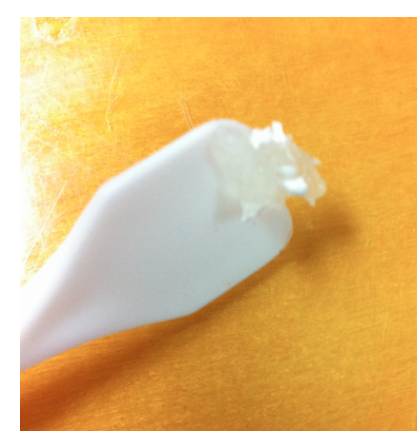

Figure 31: Nitrocellulose Lacquer. Development of a nitrocellulose lacquer made from 99\% acetone and HF 135 nitrocellulose paper using a mortar and pestal to grind and form the material.

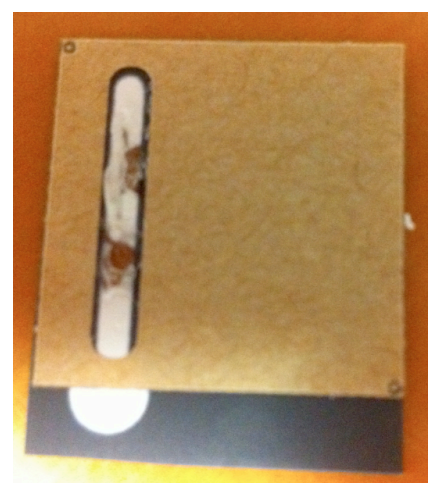

Figure 32: Application of Nitrocellulose Lacquer. Post-application of nitrocellulose lacquer onto nitrocellulose membrane surface during the manufacturing of a three-dimensional chip.

The fourth bridging layer technique was a pure piece of nitrocellulose paper used to bridge the layers. This provided uniformity of liquid to bridge between the layers as compared to the powders. This worked in providing equal distribution of sample between layers in the assembly and testing of the three-dimensional simultaneously detecting paper microfluidic chip.

\section{Testing of Three-Dimensional Chip}

Four iterations of three-dimensional chips were developed in attempting to develop a simultaneous detecting chip. For the initial design, the channel in which was furthest from the sample input detected first, followed by the second closest to the sample input, then the closest, and finally one of the detection regions did not show up 
whatsoever. The result of this chip is in Figure 33. The chip took several hours to show any detection whatsoever and between the three regions that did provide signal, it appeared over ten minutes apart from one another.

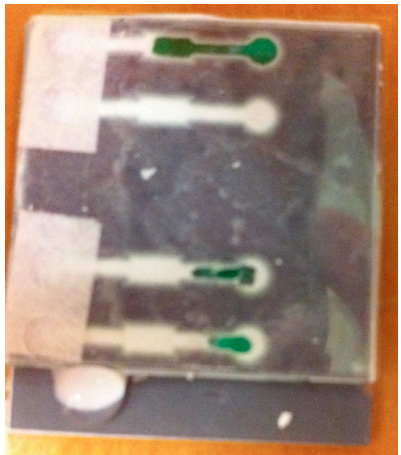

Figure 33: Results of Design Iteration 1. Non-simultaneous detection of a three dimensional chip (this is realized from three different regions with dye at different areas of the chip, and the fourth with no dye at all - occurring 10 or more minutes apart from one another).

The second and third design iterations also took a significant amount of hours to develop any signal, and when doing so were out of order and developed at completely different points in time. The detection region that showed signal first was random rather than following any pattern. An example of this may be found in Figure 34.

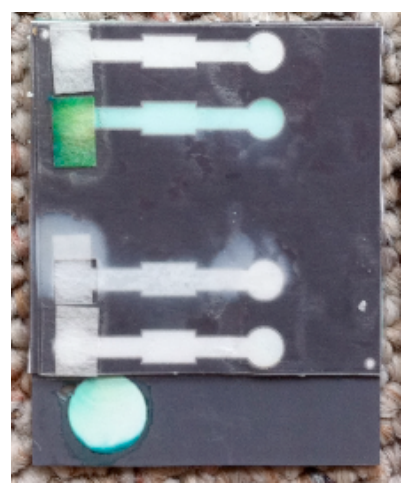

Figure 34: Results of Design Iteration 2. The second developed design with only one of four regions showing any detection.

The four design iteration yielded a result where it took around fifteen minutes to develop using a cut nitrocellulose paper bridging layer as opposed to a powder, which 
took around thirty minutes to an hour of time. Detection on the fourth chip may be found in Figure 35.

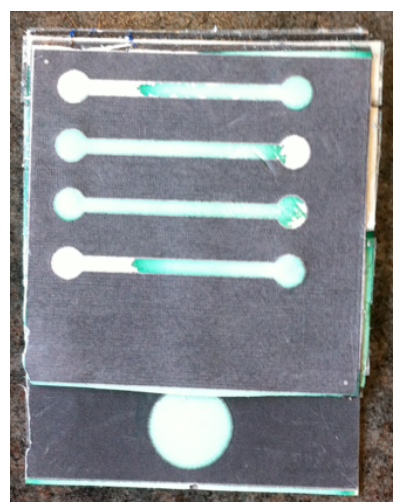

Figure 35: Results of Fourth Design Iteration. Running test of the fourth design iteration chip, noting detection within one to two minutes from the first to the last channels.

\section{Biochemistry}

The results for the biochemistry portion of this thesis were separated by the effort toward conjugating gold to antibodies, the antibody-based lateral flow assays, and the aptamer-based lateral flow assays.

\section{Conjugating Gold to Antibodies}

Following the protocol for developing a conjugated gold antibody, the solution was optically clear. Additionally, the test was run for both $\mathrm{IgG}$ as a control capture antibody and anti-VEGF as a detection capture antibody. The results yielded from this test can be found in Table VII.

\section{Table VII: Results of Conjugating Gold to Antibodies}

Results from lateral flow assay tests run for antibodies that were experimentally conjugated to 15 nanometer gold particles

\begin{tabular}{|c|c|c|}
\hline Test & Antibody & Result \\
\hline 1 & IgG & No Visual Signal \\
\hline 2 & IgG & No Visual Signal \\
\hline 3 & VEGF & No Visual Signal \\
\hline 4 & VEGF & No Visual Signal \\
\hline
\end{tabular}




\section{Monoclonal Antibody Detection}

For the initial set of antibody tests, this involved spotting down monoclonal antiVEGF capture antibodies as well as capture line IgG antibodies to the nitrocellulose surface and exposing them to a sample with VEGF along with anti-VEGF antibody conjugated with 30 nanometer gold. For the IgG antibody, its spotted down concentration remained at $1 \mathrm{mg} / \mathrm{ml}$ for the duration of experiments and was exposed to $10 \mu \mathrm{g} / \mathrm{ml} \mathrm{VEGF}$ sample concentration and $1 \mathrm{mg} / \mathrm{ml}$ colloidal gold antibody concentration. There were two tests types that were implemented - a spot test and a lateral flow assay test. Then, there were different post-treatments of the nitrocellulose pad and different ways in which the gold was applied to the test. These factors and the end result are demonstrated in Table VIII.

Like with the IgG, anti-VEGF antibody had a series of different test procedures, post-treatments of the membrane, and the application of conjugated gold antibody. The results of different combinations and test procedures taken are presented in Table IX. Additionally, with these test combinations, several different designs were used from the Methods section. They were not represented in Table IX due to a similar set of end results as well as some discoveries that were later made and explained in the Discussion section of this document. 


\section{Table VIII: IgG Test Results}

Test results for using IgG as a capture antibody for the control line.

\begin{tabular}{|c|c|c|c|c|}
\hline Test & Test Type & Post-Treatment & Gold Application & Result \\
\hline 1 & Spot Test & None & Spot & Background \\
\hline 2 & Spot Test & $\begin{array}{l}7 \text { minutes in } 1 \% \\
\text { BSA and } 5 \\
\text { minutes in } \\
\mathrm{Na}_{2} \mathrm{HPO}_{4} \& \mathrm{SDS}\end{array}$ & Spot & Background \\
\hline 3 & $\begin{array}{l}\text { Lateral Flow } \\
\text { Assay }\end{array}$ & None & Conjugate Pad & Background \\
\hline 4 & $\begin{array}{l}\text { Lateral Flow } \\
\text { Assay }\end{array}$ & $\begin{array}{l}7 \text { minutes in } 1 \% \\
\text { BSA and } 5 \\
\text { minutes in } \\
\mathrm{Na}_{2} \mathrm{HPO}_{4} \& \mathrm{SDS}\end{array}$ & Conjugate Pad & Background \\
\hline 5 & $\begin{array}{l}\text { Lateral Flow } \\
\text { Assay }\end{array}$ & $\begin{array}{l}7 \text { minutes in } 1 \% \\
\text { BSA and } 5 \\
\text { minutes in } \\
\mathrm{Na}_{2} \mathrm{HPO}_{4} \& \mathrm{SDS}\end{array}$ & $\begin{array}{l}\text { Conjugate Pad with } \\
\text { Borate + Sucrose }\end{array}$ & Background \\
\hline 6 & $\begin{array}{l}\text { Lateral Flow } \\
\text { Assay }\end{array}$ & None & Mix Gold + VEGF & None \\
\hline 7 & $\begin{array}{l}\text { Lateral Flow } \\
\text { Assay }\end{array}$ & $\begin{array}{l}7 \text { minutes in } 1 \% \\
\text { BSA and } 5 \\
\text { minutes in } \\
\mathrm{Na}_{2} \mathrm{HPO}_{4} \& \mathrm{SDS}\end{array}$ & Mix Gold + VEGF & None \\
\hline
\end{tabular}


Table IX: Monoclonal anti-VEGF Antibody Results

Test results for using monoclonal anti-VEGF as a capture antibody for the detection line.

\begin{tabular}{|c|c|c|c|c|}
\hline Test & Test Type & Post-Treatment & Gold Application & Result \\
\hline 1 & Spot Test & None & Spot & Background \\
\hline 2 & Spot Test & $\begin{array}{l}7 \text { minutes in } 1 \% \\
\text { BSA and } 5 \\
\text { minutes in } \\
\mathrm{Na}_{2} \mathrm{HPO}_{4} \& \text { SDS }\end{array}$ & Spot & Background \\
\hline 3 & $\begin{array}{l}\text { Lateral Flow } \\
\text { Assay }\end{array}$ & None & Conjugate Pad & Background \\
\hline 4 & $\begin{array}{l}\text { Lateral Flow } \\
\text { Assay }\end{array}$ & None & Conjugate Pad & Background \\
\hline 5 & $\begin{array}{l}\text { Lateral Flow } \\
\text { Assay }\end{array}$ & $\begin{array}{l}7 \text { minutes in } 1 \% \\
\text { BSA and } 5 \\
\text { minutes in } \\
\mathrm{Na}_{2} \mathrm{HPO}_{4} \& \text { SDS }\end{array}$ & Conjugate Pad & Background \\
\hline 6 & $\begin{array}{l}\text { Lateral Flow } \\
\text { Assay }\end{array}$ & $\begin{array}{l}7 \text { minutes in } 1 \% \\
\text { BSA and } 5 \\
\text { minutes in } \\
\mathrm{Na}_{2} \mathrm{HPO}_{4} \& \mathrm{SDS}\end{array}$ & Mix Gold + VEGF & None \\
\hline 7 & $\begin{array}{l}\text { Lateral Flow } \\
\text { Assay }\end{array}$ & $\begin{array}{l}7 \text { minutes in } 1 \% \\
\text { BSA and } 5 \\
\text { minutes in } \\
\mathrm{Na}_{2} \mathrm{HPO}_{4} \& \mathrm{SDS}\end{array}$ & Mix Gold + VEGF & None \\
\hline 8 & $\begin{array}{l}\text { Lateral Flow } \\
\text { Assay }\end{array}$ & $\begin{array}{l}7 \text { minutes in } 1 \% \\
\text { BSA and } 5 \\
\text { minutes in } \\
\mathrm{Na}_{2} \mathrm{HPO}_{4} \& \mathrm{SDS}\end{array}$ & Mix Gold + VEGF & None \\
\hline 9 & $\begin{array}{l}\text { Lateral Flow } \\
\text { Assay }\end{array}$ & $\begin{array}{l}7 \text { minutes in } 1 \% \\
\text { BSA and } 5 \\
\text { minutes in } \\
\mathrm{Na}_{2} \mathrm{HPO}_{4} \& \mathrm{SDS}\end{array}$ & Mix Gold + VEGF & None \\
\hline 10 & $\begin{array}{l}\text { Lateral Flow } \\
\text { Assay }\end{array}$ & $\begin{array}{l}7 \text { minutes in } 1 \% \\
\text { BSA and } 5 \\
\text { minutes in } \\
\mathrm{Na}_{2} \mathrm{HPO}_{4} \& \text { SDS }\end{array}$ & $\begin{array}{l}\text { Conjugate Pad with } \\
\text { Borate + Sucrose }\end{array}$ & Background \\
\hline 11 & $\begin{array}{l}\text { Lateral Flow } \\
\text { Assay }\end{array}$ & $\begin{array}{l}7 \text { minutes in } 1 \% \\
\text { BSA and } 5 \\
\text { minutes in } \\
\mathrm{Na}_{2} \mathrm{HPO}_{4} \& \text { SDS }\end{array}$ & $\begin{array}{l}\text { Conjugate Pad with } \\
\text { Borate + Sucrose }\end{array}$ & Background \\
\hline
\end{tabular}




\section{Polyclonal Antibody Detection}

Six different gold conjugated antibodies concentrations were developed and tested in order to determine the lowest concentration possible to still allow for visible signal strength. The visibility of the gold at the six different concentrations is represented in Table $X$. The visual test that was done can be found in Figure 36.

\section{Table X: Visual Colloidal Gold Test}

Colloidal gold test to find the optimal concentration that is the most dilute and provides strong visual signal.

\begin{tabular}{|c|c|c|c|c|c|c|}
\hline Test & $\mathbf{1}$ & $\mathbf{2}$ & $\mathbf{3}$ & $\mathbf{4}$ & $\mathbf{5}$ & $\mathbf{6}$ \\
\hline Concentration & $1 \mathrm{mg} / \mathrm{ml}$ & $0.5 \mathrm{mg} / \mathrm{ml}$ & $\begin{array}{c}0.1 \\
\mathrm{mg} / \mathrm{ml}\end{array}$ & $\begin{array}{c}0.05 \\
\mathrm{mg} / \mathrm{ml}\end{array}$ & $\begin{array}{c}0.01 \\
\mathrm{mg} / \mathrm{ml}\end{array}$ & $\begin{array}{c}0.002 \\
\mathrm{mg} / \mathrm{ml}\end{array}$ \\
\hline See Signal? & Yes & Yes & No & No & No & No \\
\hline
\end{tabular}

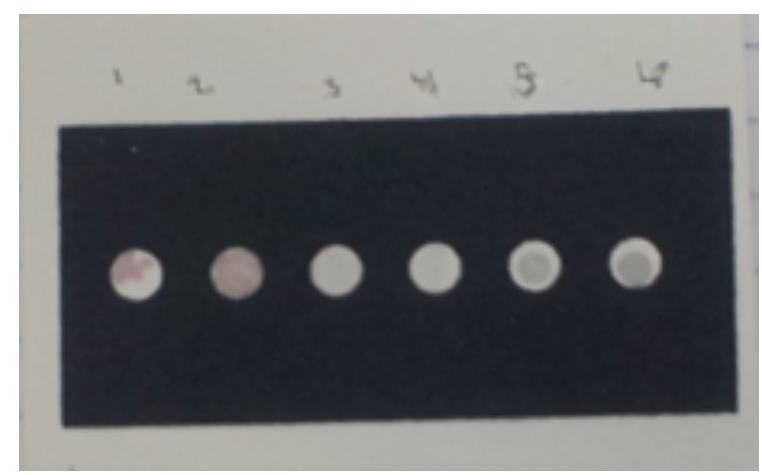

Figure 36: Gold Spot Test. Colloidal gold visualization tests using six separate concentrations. In this case test one and two (seen in the first two wells located on the left side) allow for visualization of the gold colloidal particles at concentrations of $1 \mathrm{mg} / \mathrm{ml}$ and $0.5 \mathrm{mg} / \mathrm{ml}$, respectively.

Along with the use of monoclonal anti-VEGF antibodies, polyclonal anti-VEGF antibodies were used and tested in this procedure as capture reagents in the detection region. A series of concentrations were tested (that were calculated in the Methods) and different tests, post-treatments of the membrane, and applications of gold colloidal particles conjugated to anti-VEGF antibodies were varying as well. This information, as well as the result of the test signal may be found in Table XI. 
Table XI: Polyclonal anti-VEGF Antibody Results

Test results for using polyclonal anti-VEGF as a capture antibody for the detection line.

\begin{tabular}{|c|c|c|c|c|c|}
\hline Test & Test Type & $\begin{array}{c}\text { Post- } \\
\text { Treatment }\end{array}$ & $\begin{array}{c}\text { Gold } \\
\text { Application }\end{array}$ & Concentration & Result \\
\hline 1 & $\begin{array}{l}\text { Lateral Flow } \\
\text { Assay }\end{array}$ & $\begin{array}{l}7 \text { minutes in } 1 \% \\
\text { BSA and } 5 \\
\text { minutes in } \\
\mathrm{Na}_{2} \mathrm{HPO}_{4} \& \\
\mathrm{SDS}\end{array}$ & $\begin{array}{l}\text { Mix Gold + } \\
\text { VEGF }\end{array}$ & $0.424 \mathrm{mg} / \mathrm{ml}$ & None \\
\hline 2 & $\begin{array}{l}\text { Lateral Flow } \\
\text { Assay }\end{array}$ & $\begin{array}{l}7 \text { minutes in } 1 \% \\
\text { BSA and } 5 \\
\text { minutes in } \\
\mathrm{Na}_{2} \mathrm{HPO}_{4} \& \\
\mathrm{SDS}\end{array}$ & $\begin{array}{l}\text { Mix Gold + } \\
\text { VEGF }\end{array}$ & $0.424 \mathrm{mg} / \mathrm{ml}$ & None \\
\hline 3 & $\begin{array}{l}\text { Lateral Flow } \\
\text { Assay }\end{array}$ & $\begin{array}{l}7 \text { minutes in } 1 \% \\
\text { BSA and } 5 \\
\text { minutes in } \\
\mathrm{Na}_{2} \mathrm{HPO}_{4} \& \\
\text { SDS }\end{array}$ & $\begin{array}{l}\text { Conjugate } \\
\text { Pad - } \\
\text { Sucrose \& } \\
\text { Borate }\end{array}$ & $0.424 \mathrm{mg} / \mathrm{ml}$ & Background \\
\hline 4 & $\begin{array}{l}\text { Lateral Flow } \\
\text { Assay }\end{array}$ & $\begin{array}{l}7 \text { minutes in } 1 \% \\
\text { BSA and } 5 \\
\text { minutes in } \\
\mathrm{Na}_{2} \mathrm{HPO}_{4} \& \\
\mathrm{SDS}\end{array}$ & $\begin{array}{l}\text { On Paper - } \\
\text { Sucrose }\end{array}$ & $0.5 \mathrm{mg} / \mathrm{ml}$ & None \\
\hline 5 & $\begin{array}{l}\text { Lateral Flow } \\
\text { Assay }\end{array}$ & $\begin{array}{l}30 \text { minutes in } \\
1 \% \text { BSA and } 1 \\
\text { Water Wash }\end{array}$ & $\begin{array}{l}\text { On Paper - } \\
\text { Sucrose }\end{array}$ & $0.5 \mathrm{mg} / \mathrm{ml}$ & $\begin{array}{l}\text { Possibly } \\
\text { signal - } \\
\text { most likely } \\
\text { background }\end{array}$ \\
\hline 6 & $\begin{array}{l}\text { Lateral Flow } \\
\text { Assay - Dip }\end{array}$ & $\begin{array}{l}7 \text { minutes in } 1 \% \\
\text { BSA and } 5 \\
\text { minutes in } \\
\mathrm{Na}_{2} \mathrm{HPO}_{4} \& \\
\text { SDS }\end{array}$ & $\begin{array}{l}\text { On Paper - } \\
\text { Sucrose }\end{array}$ & $0.5 \mathrm{mg} / \mathrm{ml}$ & None \\
\hline 7 & $\begin{array}{l}\text { Lateral Flow } \\
\text { Assay - Dip }\end{array}$ & $\begin{array}{l}30 \text { minutes in } \\
1 \% \text { BSA and } 1 \\
\text { Water Wash }\end{array}$ & $\begin{array}{l}\text { On Paper - } \\
\text { Sucrose }\end{array}$ & $0.5 \mathrm{mg} / \mathrm{ml}$ & $\begin{array}{l}\text { Possibly } \\
\text { signal - } \\
\text { most likely } \\
\text { background }\end{array}$ \\
\hline
\end{tabular}


Using the final concentration calculations in the Methods, a final series of polyclonal antibody lateral flow assay tests were performed to detect VEGF in sample. The results of these tests are demonstrated in Table XII.

\section{Table XII: Humidity Anti-VEGF Antibody Results}

Polyclonal anti-VEGF antibody testing using altered concentration calculations and incorporation of humidity in the chamber

\begin{tabular}{|c|l|l|l|}
\hline Test & Patterned Chip? & \multicolumn{1}{|c|}{ Gold Placement on Chip } & \multicolumn{1}{c|}{ Result } \\
\hline 1 & Yes & Conjugate Pad with Borate + Sucrose & None \\
\hline 2 & No & Conjugate Pad with Borate + Sucrose & None \\
\hline 3 & Yes & On Paper - Sucrose & None \\
\hline 4 & No & On Paper - Sucrose & None \\
\hline
\end{tabular}

\section{Aptamer Testing}

Initially aptamers that were tested were pure single strands of DNA that were spot blotted onto the nitrocellulose membrane and treated accordingly. Table XIII presents the types of tests and post-treatments done to the nitrocellulose membrane and the result of the test.

\section{Table XIII: Pure Aptamer Results}

Results from testing for detection of VEGF in sample using aptamer-based capture reagents

\begin{tabular}{|c|l|l|c|}
\hline Test & \multicolumn{1}{|c|}{ Test Type } & \multicolumn{1}{c|}{ Post-Treatment } & \multicolumn{1}{c|}{ Result } \\
\hline 1 & Lateral Flow Assay & None & No Visual Signal \\
\hline 2 & Lateral Flow Assay & $\begin{array}{l}7 \text { minutes in 1\% } \\
\text { BSA and 5 minutes } \\
\text { in } \mathrm{Na}_{2} \mathrm{HPO}_{4} \& \text { SDS }\end{array}$ & No Visual Signal \\
\hline 3 & Spot Test & None & No Visual Signal \\
\hline 4 & Spot Test & $\begin{array}{l}7 \text { minutes in 1\% } \\
\text { BSA and 5 minutes } \\
\text { in } \mathrm{Na}_{2} \mathrm{HPO}_{4} \& \text { SDS }\end{array}$ & No Visual Signal \\
& & & \\
\hline
\end{tabular}

Again aptamer tests were completed. This time aptamers were biotinylated on one end to allow for further adhesion to the nitrocellulose membrane in conjunction with 
streptavidin. After running the test with the VEGF solution, the chip revealed a pink hue from the gold colloidal antibodies. This implied detection due to the definitive shape of one of the two detection wells. An image of this may be found in Figure 37.

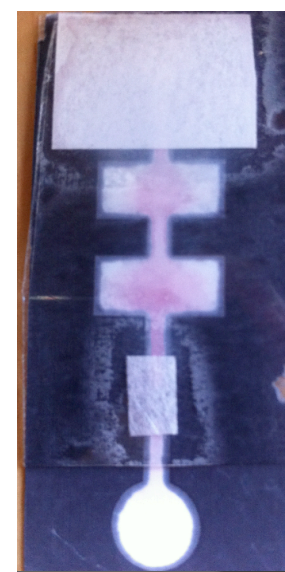

Figure 37: Biotinylated Aptamer. Resultant test of the biotinylated aptamerbased detection lateral flow assay for $50 \mathrm{ng} / \mathrm{ml}$ VEGF sample. 


\section{Discussion}

The resultant of this thesis project showed many characteristics exhibited by nitrocellulose membrane, the resultant of developing a three-dimensional microfluidic chip, and the comparison of aptamers to antibodies as possible reagents of detecting VEGF in sample. The discussions of these results are presented.

\section{Characterization}

The characterization process of this project displayed important information for further understanding the three-dimensional chip development for simultaneous sample detection. These steps furthered the understanding of wicking time and the visual characteristics of nitrocellulose membrane. Explanations behind the characterization results were important for understanding not only the results of the three-dimensional chip design and development process, but for future paths regarding $\mu \mathrm{Pad}$ development.

\section{Channel Widths and Wax Reflow}

Initially, there were sets of different linear and circular channel widths that were designed in SolidWorks. Although this limits-of-detection aspect of this thesis was taken over by a fellow student, it has much importance toward this thesis project and others regarding lateral flow assays and paper microfluidics. First of all, by analyzing the channel widths that can or cannot maintain sample, it would be important in determining the optimal width one can design in such a device. This controls the speed of the sample as well as how much sample can possibly be used in order to wick through the device's entirety. This minimizes resources and lowers cost. Additionally, by analyzing the circular designs, it would further explain why and how curvature changes wicking time throughout the paper-based device. 
For studying the limits of detection by developing a chip with $225 \mu \mathrm{m}, 250 \mu \mathrm{m}$, $275 \mu \mathrm{m}$ and $285 \mu \mathrm{m}$ channel thicknesses, it was seen that for the first two widths, wax entirely covered the channels. For the latter two channels, the thicknesses could be visualized, but once placed under heat to impregnate wax into the channels, wax somewhat reflowed back into the open areas of nitrocellulose. This technique ended up to be discarded for this thesis project. The main reason was due to the fact that the chips being developed were to be visualized by the user. With channels this small, sample travel throughout the channel would have to be determined through the use of microscopes, which this project wanted to avoid the use of. The purpose of developing these chips were to allow naked eye visualization from somewhat that was or was not trained in healthcare diagnostics.

For the tests comparing bibulous paper to the $0.45 \mu \mathrm{m}$ pore size nitrocellulose membrane for wicking times with designs, the test ended up to yield minimal results. The bibulous paper had quite a bit of wax reflow when exposed to heat. The properties of bibulous paper, which is often made of cotton, absorbs liquid at a high rate, so as the wax was heated and melted, the blot paper allowed for flow of the wax into its channels. Additionally, when sample was placed onto the paper it wicked at an extremely high rate (under a minute from input to development of sample). The $0.45 \mu \mathrm{m}$ pore size nitrocellulose on the other hand, when spotted on the surface, did not wick whatsoever, even after sitting on the membrane for thirty minutes. The reason why this membrane could not wick solution was due to the fact that it had no added surfactant to the material [16]. Naturally, nitrocellulose is a hydrophobic material; therefore as the aqueous 
solution was placed onto the membrane, the droplet held a high contact angle on the paper's surface and did not allow for wicking through the membrane.

\section{Optimal Channel Characteristics}

The initial testing of optimal channel widths reviewed $0.8 \mathrm{~mm}, 1.6 \mathrm{~mm}$, and 2.4 mm widths. These were chosen due to the fact that they could be visualized by the naked eye. After testing it was seen that the $1.6 \mathrm{~mm}$ channel width was the best for wicking, followed by $2.4 \mathrm{~mm}$, then $0.8 \mathrm{~mm}$. The thinnest channel width had trouble wicking from initial input to detection. Therefore, this channel width was not used for the duration of these studies. Although the $1.6 \mathrm{~mm}$ channel width had a better wicking time than the 2.4 mm width, both were used throughout the project because the time difference for the two was not significant enough to solely use one particular channel width. Additionally, the $2.4 \mathrm{~mm}$ size allowed for better visualization by the user than the $1.6 \mathrm{~mm}$ channel width.

\section{Design Combinations and Wicking Times}

The four assumptions that were considered when analyzing the data were normality, constant variance, independence, and model adequacy. The normality was violated, as the deleted residuals line curved away from the diagonal line four different times; this was found in the "Normal Probability Plot" in the four-in-one "Residual Plots for Time" showing that this assumption was violated. The independence assumption, on the other hand, was not violated, as the design was randomly assigned and there was no time series data. For model adequacy, this was also not violated, because the initial model considered every single interaction possible for all of the factors. Finally, for equal variance the "Versus Fits" plot was used. This showed a violation in the assumption due to the large differences in the spread of data in the plot however because only two 
replicates were completed. If there had been more than two replicates of the experiment the data would have shown a more consistent variance among the results. Additionally, looking at the Cook's distance for this model, there were no values larger than 0.5 , showing that there was no moderate or extreme influence in the data. These Cook's distances can be found in Appendix A.

The data was then transformed to a logarithmic version of the response variable in an attempt to fix the violated assumptions. Like with the initial model, the four assumptions considered were normality, constant variance, independence, and model adequacy. By transforming the data using a logarithmic function on the response variable, this improved the normality. In the Normal Probability Plot, the data remained close to the diagonal line on the plot. The independence assumption remained non-violated because of the random assignment of the treatments and non-time series data. The equal variance, although showing a large spread of data in the Versus Fits graph, is not violated due to the fact that only two replicates were done in the experiment. Additionally, the Cook's distance was analyzed to see if there was any influence in the data. There were no Cook's distances of beyond 0.5 , indicating that there were no moderate or great influential points in the data. This can be found in Appendix A.

Analyzing the experiment's residual plots and finding that various assumptions were violated indicated that there were problems that needed to be fixed. Instead of dropping high-order, insignificant interactions (which would cause a violation to the model adequacy assumption) transformations of the data was attempted. The initial transformation done was the $\log (\mathrm{y})$ function. According to the transformation process, it is most beneficial to begin at the log function and progress towards square roots to 
squares to cubes, and so on (along with their inverses). The response (y) variable, time, were transformed using the Calc function in Minitab, labeling the corresponding column in the dataset $\log ($ time $)$ and using the log base 10 function. The values were calculated and an identical ANOVA test was run as before, now using Log(time) as the response variable and the five factors (and all possible interactions) as the predictor variables.

Once all assumptions were found to be not violated after using the $\log$ (time) transformation, the factors and interactions could be interpreted. A total of 31 factors/interactions were generated through the ANOVA test. These were interpreted using an overall significance level of $10 \%$. $10 \%$ was chosen, as opposed to $5 \%$, due to the fact that this was a student run experiment with two replicates, and there was a large amount of interactions. A $10 \%$ overall significance level with 31 factors/interactions yields a $.323 \%$ individual significance level $(0.10 / 31=0.003226)$. The ANOVA test generated p-values specific to each of the 31 factors/interactions. These individual pvalues were compared to the individual significance level of $.323 \%$. These comparisons were done in order of the most complex interaction (all five factors) to the simplest main effect (any one of the five factors). As soon as an interaction proved to be significant (contained a p-value less than the significance level, 0.00323) interactions containing the same factors as the significant one could be ignored. The first significant interaction found (p-value of 0.001 ) was the interaction containing Backing*Curving*Porosity*Width. This means that any other interactions (or main effects) containing only these factors need not be interpreted. The next interaction (without only these factors) found to be significant (p-value of 0.002) contained Backing*Size*Width. This interaction could be interpreted as significant due to fact that 
size was included, while it was absent from the first significant interaction. Now that all factors have been accounted for in significant interactions, multiple comparisons could be done for these significant interactions.

In order to analyze the comparisons amongst each of the factor levels within the two significant interactions, the method of Tukey intervals were used. Tukey intervals were chosen over Dunnett intervals because there was no control group in this study. They were chosen over Bonferonni intervals because Tukey intervals are more powerful and still control for Type I error. For this study, the endpoint was a proof of concept, making power a high priority. However, controlling the experiment wide Type I error rate was also crucial, and is the reason Tukey was chosen over Fisher. Fisher may have higher power than the other intervals, but it does not control the Type I error.

Tukey intervals were run for Backing*Size*Width and Backing*Curving*Porosity*Width (full output of these comparisons can be found in Appendix A). These comparisons generated the mean responses (mean $\log ($ time)) for each of the combinations of factor levels within the interaction. These means were arranged from largest to smallest. The intervals indicated any significant differences found between various combinations of the factor levels. These significant differences were determined by finding the intervals that did not contain the number zero. The combinations corresponding with these intervals are then compared to each other using a line/letter chart (in order of decreasing means). Combinations that do not touch lines or contain the same letter are considered to be significantly different from each other.

Based on the letter chart results (found in Appendix A) of the significant interaction of Backing*Curving*Porosity*Width, the following interpretations it was 
concluded that:

- At the overall 10\% significance level, unbacked-curved-hi-wide chips have significantly slower median wicking times than the rest of the chip material and design combinations.

- Backed-curved-lo-narrow, unbacked-straight-lo-wide, backed-curved-lo-wide, backed-straight-lo-narrow, and backed-straight-lo-wide do not show significant differences in their median wicking times.

- Backed-straight-lo-wide has a significantly faster median wicking time than all of the combinations ranging from unbacked-curved-hi-wide (mean log wicking time of 2.8) to unbacked-straight-lo-narrow (mean log wicking time of 1.5).

Additionally, based on the letter chart results (found in Appendix A) of the significant interaction of Backing*Size*Width, the following interpretations were concluded:

- At the overall $10 \%$ significance level, backed-short-wide has a significantly faster median wicking time than the rest of the combinations in this interaction.

- Combinations ranging from unbacked-long-narrow (mean log wicking time of 2.0) to unbacked-short-narrow (mean log wicking time of 1.7) are not significantly different in median wicking time.

In these interpretations, the use of median wicking time is used because of the fact that a transformation was done on the data. The means generated in the comparisons are the means of the $\log ($ time $)$. This means that ten raised to these values would give the actual mean wicking time. 


\section{Wicking Time - Conjugate Pad vs. Membrane Paper}

For the experiment involving wicking time between four identical channels on plain nitrocellulose membrane, there was a standard deviation of 1.25 seconds and an average time of 42.73 seconds for the sample to reach from beginning to end. The times were not significantly different from one another for the four different tests. This shows that the nitrocellulose material was not significantly different from one area to another. This was important for understanding that a simultaneous detection chip could be developed.

On the other hand, the four identical channels that contained a conjugate pad had a standard deviation of 203.9 seconds and an average time of 432.85 seconds. This significant difference between the different channels was firstly due to the fact that the conjugate pads could not be cut to an identical size every single time. Because the fiberglass pad soaks up a lot of liquid before saturating and sample leaving the pad, any slight size difference can significantly change the time. Secondly, the fiber orientation in the fiberglass pad changes dramatically throughout, and there was no way to account for the changes in each pad. Therefore, the addition of this element does not allow for somewhat simultaneous results and instead dramatically varies wicking time.

Comparing the channels that did or did not contain a conjugate pad for wicking time, a t-test was used. The end result was a p-value of 0.0314 , a statistically significant value (because it was less that 0.05 ). This means that the null hypothesis was rejected, that stated that there was no significant difference between wicking times of the experiment containing and not containing a conjugate pad. This comparison was important for understanding that when a three-dimensional chip contains an additional conjugate pad throughout the platform, it will significantly add to the time it takes to 
reach detection. The experiments were also important in determining that it was unlikely that simultaneous detection would occur between different detection regions for all threedimensional platforms when a conjugate pad was used in the apparatus.

\section{Imaging}

Although the SEM technique that was used was not for quantifiable purposes of this project, it was important in understanding the way in which the membrane appeared and changed with the addition of wax. It was seen that the pores on the $0.45 \mu \mathrm{m}$ membranes were significantly smaller than those on the HF 135 membrane. However, no noticeable addition of surfactant on the HF 135 membrane as compared to the raw 0.45 $\mu \mathrm{m}$ pore sized nitrocellulose could be determined.

When compared side-by-side there is no noticeable difference between HF 135 imbedded with wax versus a bare surface. This means that the wax settles into the nitrocellulose membrane to an extent in which it is visually undetectable at a high objective. Therefore, the wax sits in and around the nitrocellulose rather than "clumping" along the surface of the membrane.

At a less magnified view $(\sim 100 \mathrm{X})$ the seam between bare and wax coated nitrocellulose can be detected in the SEM. The wax appears slightly darker but, again, the seam is relatively difficult to determine. This reinforces the fact that wax is relatively undistinguishable when impregnated in the nitrocellulose at a microscopic level. However, the color of the wax can be seen at a macroscopic level by the human eye. 


\section{Development of a Three-Dimensional Chip}

After properties of the nitrocellulose were determined, aspects of the manufacturing and development of the three-dimensional chip were defined to eventually create a working simultaneously detecting $\mu$ Pad. Through experimentation optimal materials and manufacturing processes were chosen, as well.

\section{Bridging Layer}

The initial bridging layer method was to use a powdered form of nitrocellulose. The first powder development attempt was using the $0.45 \mu \mathrm{m}$ pore size nitrocellulose in conjunction with acetone to create the appropriate consistency. However, after much effort toward grinding the materials to make a powder, very little material was produced. This method seemed relatively cumbersome for such a minor yield of nitrocellulose powder. Because of this, the bridging layer method for this particular membrane type was discarded.

The next powder technique with grinding HF 135 or 180 membranes with acetone easily created powder. When it was dried out, however, the powder pieces hardened and came in an array of sizes. The stiffness of the particulates often led to a difficult manufacturing process of the three-dimensional chip, as the powder pieces would often cut through the nitrocellulose membrane and make the designs unusable for testing.

Additionally, the use of acetone as a way to make powder seemed questionable to use for a true lateral flow assay device, in case the reagent had an adverse affect on any of the reagents that could possibly be used. Likewise, when water was used instead of acetone to grind the nitrocellulose membrane, the process of developing a powder was cumbersome to produce and when dried still had the issue of stiff particulates ripping the nitrocellulose membrane layers. A way to resolve this issue was to place the powder 
while still damp into its appropriate bridging areas. However, this method was still not preferable because it was still a messy method during the manufacturing process. The powder was not uniform within each designated bridging layer and created an issue for designing a simultaneous detecting chip. Therefore, the idea of a powder bridging layer was discarded for this thesis.

The lacquer that was created from a drugstore nail polish remover (advertised to be $100 \%$ acetone) when ground up with nitrocellulose membrane produced a lacquer rather than a powder bridging layer. A lacquer would be advantageous in terms of applying the bridging material down would be better controlled by the manufacturer. However, upon applying the lacquer down to the surface, the nitrocellulose membrane would disintegrate due to the acetone presence in the material.

The final bridging layer was simply cutting out the nitrocellulose membrane to fit into the areas where bridging of the sample would take place. This was the preferred technique used for developing a three-dimensional simultaneous detecting chip. The characterization step that compared nitrocellulose wicking times within the four channels showed that there were no major differences in wicking time between different areas and pieces of nitrocellulose. This showed that using a fit-to-size piece of nitrocellulose membrane would be a relatively good bridging layer material for simultaneous detection due to the relatively isotropic properties of the membrane. Additionally, it was not as difficult to use during the assembly process. Although, cutting the pieces by hand was a little more difficult for smaller bridging areas. However, it was later discovered that specialized hole punches could be purchased and used to cut out the nitrocellulose membrane into specific shapes for the bridging layer. This made the cutting process much 
easier and uniform between pieces of nitrocellulose and aided in a more streamlined process in the manufacturing of these three-dimensional chips.

\section{Testing of a Three-Dimensional Chip}

The initial two designs of the three-dimensional chip commonly did not allow for simultaneous detection of sample. After running a statistical characterization step regarding channel curvature, it became apparent that a main problem with the design related to the fact that there were two curved patterns within the chip designs. Although it was originally thought that curved lines would take the same amount of time to wick as linear channels, given the same path length and channel width, this ended up to not be the case.

The next design used powder as a bridging layer between the nitrocellulose membranes. This may have been a possible issue behind the non-simultaneous detection in the four detection wells. This would be due to the fact that the powder particulates were not equal in size and the process in which the chips were developed, more powder may have been placed in some bridging areas over others. This would limit or add to the wicking time per each channel.

The final design was preferable over the first three. The final design used nitrocellulose membrane in its sheet form as a bridging layer. Experiments were done where a conjugate pad was placed into the three-dimensional chip versus no conjugate pad whatsoever. Like with the two-dimensional test that was formed, it was important to see how much of an effect the addition of the fibrous conjugate pad would have on the wicking times of the four separate channels. Without the conjugate pad, the four 
detection regions showed signal within one to two minutes from the first to the last channels. On the other hand, when using conjugate pads, the results showed up within ten minutes of one another in the detection region. This once again proved the anisotropic nature of the fiber orientation of the conjugate pad and the inability to truly produce a simultaneous detecting chip of a multi-analyte sample.

\section{Biochemistry}

The biochemistry aspect of this project yielded visual detection for the biotinylated aptamer capture reagents. However, all other capture reagents showed no visual signal. The observations and explanations behind these results are described.

\section{Conjugating Gold to Antibodies}

It was seen that the solution in which gold was placed with antibodies was

entirely clear. Without seeing this red color (from the gold nanoparticles) it was assumed to either not bind to the anti-VEGF or to just be far too dilute for any visualization. Without visualization in solution, it is unlikely that any detection will be noted for the sample specimen. This idea was discarded solely for the fact that there was minimal information on the conjugation process and because there were options to purchase antiVEGF antibodies that were conjugated to gold already. Additionally, the colloidal gold that was on-hand was smaller than the standard colloidal gold used for diagnostic applications, which is around 30 to 40 nanometers. 


\section{Monoclonal Antibody Detection}

For the initial set of antibody tests, where the gold was pre-conjugated to antiVEGF antibody, there were several issues of note. Firstly, the IgG that was purchased as a detection antibody was noted to not be of use to pair with the anti-VEGF antibody. This was due to the fact that the IgG was rabbit anti-mouse, and the antibody attached to gold was a Rabbit anti-VEGF-A. Although the IgG states that the antibody reacts with heavy chains of mouse $\operatorname{IgG}$, it may also react with immunoglobulins from other species. However, typically, in order for the IgG to have bound to the anti-VEGF it would have to either be an anti-Rabbit, or the VEGF antibody would have to be a mouse species. Therefore, these experiments may not have worked due to the mismatch in species of the antibody origin. However, when the IgG was initially purchased, it was intended to match with the mouse anti-VEGF antibodies that were purchased in the gold conjugation experiment, and would have had appropriate binding to one another.

With the first set of anti-VEGF antibodies that were purchased, it was noted that there was still no signal for the lateral flow assay tests containing VEGF sample. After contacting the vendor and doing a BLAST on the antibodies and their binding properties, it was seen that the antibodies only bound to a very specific epitope of the $\mathrm{VEGF}_{165}$ isoform. This created competitive binding between the capture and the detection antibody. This inhibited two antibodies from binding to the protein and producing a sandwich ELISA-like assay. Therefore, a new capture antibody needed to be used in order to allow for the assay to work.

\section{Polyclonal Antibody Detection}

The next anti-VEGF antibody that was purchased was raised against the entire VEGF165 sequence and theoretically would allow for binding anywhere on the VEGF 
protein. Therefore, the gold conjugated antibody could bind to the specific epitope of the protein and cause no competitive binding for the capture antibody. However, no results were found from doing any of these antibody tests. There could be several issues regarding what could be the problem behind this. Firstly, it could be due to a lack of attachment of the antibody to the nitrocellulose membrane. The lack of anchoring could be due to the antibodies not having been viable any longer. This would have most likely happened due to repeat freezing and thawing cycles of the antibody during the testing process. Additionally, it could be that the anti-VEGF antibody was not robust enough to remain attached to the membrane meaning that no initial binding was occurring using the spot blot biochemistry technique, or that it was washing off during the blocking step with $1 \%$ BSA of the washing step with the sodium dodecyl sulfate and $\mathrm{Na}_{2} \mathrm{HPO}_{4}$. The next option could be that the polyester backing of the nitrocellulose could be interfering with the anti-VEGF's capability of attachment to the membrane. Likewise, even after attempting to add humidity into the drying chamber of the antibody, there still could have not been a sufficient amount of liquid present in the area, which caused the evaporation of antibody from the surface [55]. In addition to the concept that antibodies may not have bound to the surface of the nitrocellulose, another possibility could be that there was an insufficient concentration of any reagents for the test. In the antibody spotted on the membrane were too high, it could be clogging the pores, and if it were too low, then it may not be picking up enough VEGF and colloidal gold with the antibodies to show an appropriate amount of signal. Another possibility is that the sample is moving too quickly through the chip, which would make the antibodies unable to appropriately bind to the proteins. This could be altered through changing channel widths of having less porous 
paper. The antibodies could also not be appropriate to use in lateral flow assays.

Commonly, antibodies will work well in a Western Blot application but not a lateral flow assay due to how the antibodies are extracted. Antibodies, when made for certain applications, are extracted in a way where they lose specificity for their appropriate proteins and can no longer recognize them post-extraction. Another issue is that the membrane may be blocked too much and could inhibit the signal of the proteins and the gold.

A final reason could have to do with the binding kinetics between VEGF and antiVEGF antibody. The Surface Plasmon Resonance (SPR) thesis project headed by Heather Hedeen in the Biomedical and General Engineering departments at Cal Poly showed poor binding kinetics between the antibody and protein in her project. Binding kinetics are often analyzed by the dissociation constant. This value quantifies the binding strength between ligands and receptors. The higher the value of this is, the lower the binding strength between the analyzed proteins. After completing three SPR trials using the polyclonal anti-VEGF antibodies, her data yielded dissociation constant values of 121.9 $\mu \mathrm{M}, 126.6 \mu \mathrm{M}$, and $16.8 \mu \mathrm{M}$. On the other hand, her dissociation constants for her aptamer (after completing two experiments with SPR) were $20 \mathrm{nM}$ and $28 \mathrm{nM}$. The aptamers, because of their smaller value, show significantly stronger binding to the VEGF than the antibodies. This corresponds to the Results where some of the polyclonal antibodies were regarded to having "possible signal - most likely background." Since the signal was so faint, it would not be realistic to use in diagnostics. Additionally, because background noise was so common throughout the antibody tests, it was likely that this faint signal could be just that. Therefore, because antibodies yielded no visual result of a 
positive test, and the aptamers did, this aided in explaining the possibility as to why no signal was produced in the tests.

Regarding the conjugate pad - there was something interesting to note. As recommended in correspondance with a biochemistry representative at Millipore, when the gold detector particle appears to be deeper purple in color, this means that the antibody and gold were aggregating [56]. This aggregation affects the end detection result, as the antibodies and gold are adhering to each other, and eventually are unable to bind to the capture reagent. When placing the conjugate pad in the oven, with or without humidity, this deep purple color appeared. This result implied aggregation. However, when aggregation does not occur, the color of the solution trends between a pink and maroon. When the conjugate pad was dried in a humid chamber at room temperature, this color was maintained, implying no aggregation.

\section{Aptamer Testing}

The initial aptamer capture reagents showed no detection when exposed to VEGF protein. However, the aptamer sequence was only twenty-eight base pairs long. Commonly, visual signal demonstrated by aptamers happens when the oligonucleotide is larger than 500 base pairs [56]. However, after adding a protein to the end of the single stranded DNA, this allows for better binding kinetics and allowance for visual signal. Therefore this thesis coated the surface with streptavidin and then a biotinylated VEGF aptamer. From there, signal was seen. Therefore it can be determined that for the VEGF aptamer, the addition of a protein to the end of the strand aids in further recognition of signal with the colloidal gold antibody for detection. 
The signal itself was relatively weak as compared to a signal created by lateral flow assays on the market (i.e. a pregnancy test). There could be several reasons behind this. One is that the area in which the lateral flow assays were developed were not necessarily in a sterile environment. In many cases a diagnostic tool is created in a clean room in order to limit the amount of dust particulates or proteins in the area. Because nitrocellulose has a high affinity for protein binding, there is a possibility that there could have been competition on the paper between the streptavidin that was coated onto the paper and what was already on the nitrocellulose to begin with. By pre-treating the membrane with a cleaning solution this could be prevented or by processing the reagents and tests in a clean room. Additionally, through a series of iterations and comparative testing, a more optimal procedure for aptamer application could be determined to strengthen the signal. Likewise, the process of developing the wax chips through the printer and then the oven could have an affect on the properties of the nitrocellulose. From experimental observation, the way in which the antibodies during the spot blot protocol spread on the paper were noticeably different between the wax treated paper and raw nitrocellulose. It was noted that the processed chips had a significant amount of spreading of the materials over that of the non-processed membrane. 


\section{Conclusion}

This thesis consisted of three main objectives. The first was to characterize the different components that were being used to form a paper microfluidic chip, the second was to design, manufacture, and test a three-dimensional chip for simultaneous detection, and the third was to compare the use of antibodies and aptamers as capture reagents in detecting the protein, VEGF.

From analyzing the data from the characterization process, it was seen that the ideal channel width for nitrocellulose it $1.6 \mathrm{~mm}$ for the fastest wicking time. In addition, it was noted that the combination of backing on nitrocellulose membrane, a linear channel, a higher porosity, and wide channel design had the fastest wicking time. The other significant interaction determined was that between the backing of the nitrocellulose membrane, the shorter channel length, and wide channel design had the fastest wicking time for the nitrocellulose membrane. Finally it was noted that channels patterned on different areas of the nitrocellulose membrane will not have significantly different wicking times, showing that the material has similar fiber orientation throughout its entirety. On the other hand, if a conjugate pad is added to the channels, the wicking time will be significantly longer for the channels, as well as significantly different between each conjugate-pad test. This shows that each conjugate pad has a significantly different fiber orientation and will add to complications in the possibility of simultaneous detection times.

Analyzing the data obtained from the three-dimensional simultaneous detecting chip, it was determined that the bridging layer technique that was more advantageous for the equal spreading of sample between layers and the easiest for manufacturing was that 
of a cut-to-size nitrocellulose membrane. Additionally, when developing a threedimensional chip, a design that contained four layers of linear-based channels produced simultaneous detection of an analyte in four detection regions. However, the simultaneous detection only occurred when a conjugate pad was not involved in the chip (i.e. all four detection regions showed detection within one to two minutes). Once the conjugate pad was introduced to the three-dimensional chip, this significantly altered the detection time of the chip as well as introduced the inability to allow for simultaneous detection.

For the biochemistry, the antibodies used as capture reagents to detect VEGF in sample were not viable for detection. Despite many efforts and changes made to protocol, there was no significant visual detection of VEGF made in the lateral flow assay. However, a project dealing with proof-of-concept analyzing the antibody binding kinetics of anti-VEGF to $\mathrm{VEGF}_{165}$ (using surface Plasmon resonance) showed poor binding between the two. This would explain a lack of visual signal from the lateral flow assay. However, using aptamers with a biotin end group did show visual signal. Once again with the proof-of-concept project that used aptamers also provided data to agree with this as the aptamer provided better binding kinetics to the VEGF than the antibody. Therefore an aptamer may be a feasible capture reagent to use in a lateral flow assay.

\section{Future Work}

As the field of paper microfluidics comes to fruition, there are many future steps that could be taken with this project and the field in general. 


\section{Characterization}

In terms of characterization, there are many more steps that could be established. One important aspect would be to conduct further studies on limits of detection. For example, looking at how small of a channel could be designed that would allow sample to pass through for detection and how it would provide importance to diagnostics. Likewise, studying the optimal amount of time nitrocellulose and printed wax should melt in the oven in order to prevent reflow of the wax and to maintain the exact design implemented on AutoCAD.

Additionally, other materials should be analyzed for their use in paper microfluidics. Nitrocellulose itself is not a very strong material and can significantly hinder the time and viability of these devices. Membranes made of materials like nylon are sturdier. So the characterization of something like nylon could aid in a more robust device.

\section{Three-Dimensional Device}

Although there was initial work done to prove that a three-dimensional device could be created there are many more steps that could be taken for the improvement of a simultaneous detecting device. For example, when the conjugate pad was added to the device itself, it provided inability to produce a simultaneous detecting chip. If alternate materials were used, simultaneous detection could be possible.

Likewise, the manufacturing process of these chips could be more streamlined than they currently are. A tool was developed on SolidWorks in this project that could be created via rapid prototyping (see Appendix $C$ for manufacturing drawings). This tool would aid in the alignment of the nitrocellulose membrane layers and tape rather than

doing it by hand. Also, costs could be cut to the manufacturing process. A demonstration 
of the assembly of such a device may be found in Figure 38. Assembled together, there is a base that would perfectly fit the chip layers. The tape and paper layers would have designed markings on two opposite diagonal corners that would imply their immediate position on the device. In the base there would be two sewing needles that were press-fit into the base to allow aligned assembly of the layers. Where the markings are made on the layers would be where the needles puncture the material in order to aid in alignment. Finally, a top block would be placed upon the chip in order to compress the layers together into one device.

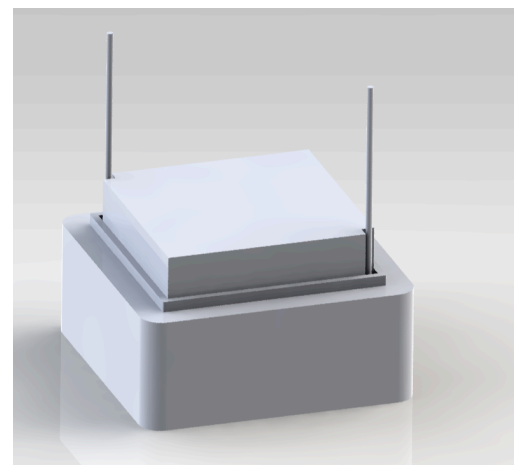

Figure 38: Depiction of Assembly Tool. Tool in its compressed assembly to allow for the manufacturing of a multi-layer paper microfluidic device.

Currently, the double-sided tape layers are cut using a laser cutter. However, this could easily be done by designing a stamping device that cuts the patterns that the chip would need. This tool could be sent to a developing nation or a low-resource development center where the tape layers could be manufactured, rather than relying on a more expensive tool like a laser-cutter.

Additionally, the designs in the detection regions of this chip could be more distinct for the end user. For instance, with the three-dimensional chip that was developed in this project, the four detection regions were not distinct from one another. Thinking about whom the device is catered toward, it would be easy to develop detection regions 
that are different shapes or patterns in order to determine the different regions that analytes are detected in. Initial designs were developed for this and can be found in the Appendix $C$.

\section{Biochemistry}

Although this thesis could not provide visual signal using antibodies at the detection region of the two dimensional platform, it would be important to still determine that this can or cannot be done for the detection of VEGF. The addition of a control line antibody would be of use as well. Then this could provide a more comparable result between aptamers and antibodies as capture reagents. Likewise, using these reagents in a three-dimensional chip would be useful to see if the idea of using a multi-layer chip for detection is feasible.

If paper microfluidics were to be implemented for clinical use, it would be important to be able to detect multiple biomarkers in a raw biological sample. Therefore, it would be of use to look into materials that could filter raw sample through a test and allow for detection. Likewise, if multiple biomarkers are to be detected, the use of latex beads rather than colloidal gold could be implemented. Latex beads come in multiple colors and would provide a clear difference in color for a positive signal.

Additionally, in the developing world, although there may not be much medical equipment that can be used by those in low-resource regions, many people have cellular telephones. In recent years there has been a push in the medical community to develop algorithms for mobile phones to analyze and process images [3]. If the user were unable to determine the result of a lateral flow assay test, an image analysis program could be developed for the mobile phone that would process a picture taken by the user and give 
the result of the test. Another option could be a potential reader device that enabled a chip to be placed into the device and analyzed to determine the diagnostic result. A concept was designed on SolidWorks and may be found in Figure 39.

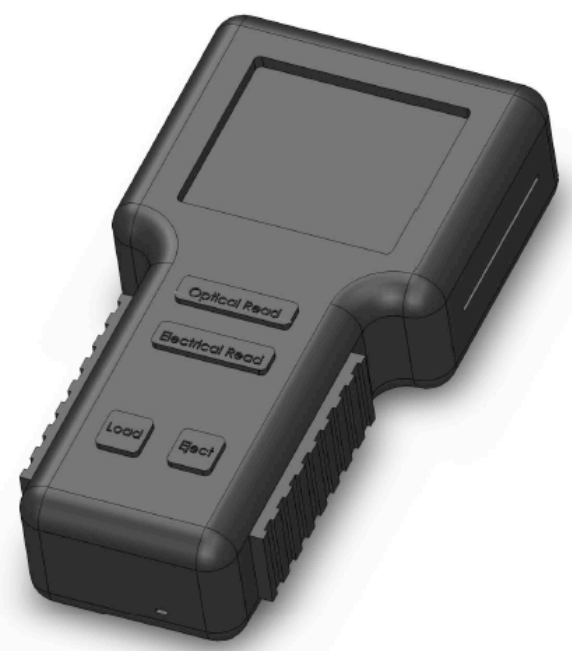

Figure 39: Conceptual design of diagnostic chip reader. 


\section{List of References}

1. Andres W. Martinez, S.T.P., George M. Whitesides, Emanuel Carrilho, Diagnostics for the Developing World: Microfluidic Paper-Based Analytical Devices. Analytical Chemistry, 2010. 82(1): p. 3-10.

2. Darren J. Carter, R.B.C., Lateral flow microarrays: a novel platform for rapid nucleic acid detection based on miniaturized lateral flow chromatography. Nucleic Acids Research, 2007. 35(10): p. 1-11.

3. Paul Yager, G.J.D., John Gerdes, Point-of-Care Diagnostics for Global Health. Annual Review of Biomedical Engineering, 2008. 10: p. 107-144.

4. Zhichao Zhou, H.G., Krishna Reddy, Xiaoping Duan, Eugenie Kleinerman, Role of VEGF165 and VEGF189 in the growth and tumor vessel expansion in Ewing's sarcoma, in American Association for Cancer Research. 2005: Houston, TX.

5. Magnus Blomsrtöm, R.E.L., Mario Zejan, What Explains Developing Country Growth?, in NBER Working Paper Series. 1992, National Bureau of Economic Research: Cambridge. p. 1-31.

6. A.C. Kelley, The Human Development Index: "Handle with Care". Population and Development Review, 1991. 17(2): p. 315-324.

7. K. Annan, Address to the Tenth United Nations Conference on Trade and Development, in United Nations. 2000: Bangkok, Thailand.

8. B.O.F.a.J Bauer, Part 2: New challenges with new approaches. Developing highly sensitive, more reproducible lateral-flow assays 2006 [cited 2012 March 9, 2012].

9. $\quad$ Agustin Benito, J.M.R., Poor Accuracy of Rapid Diagnostic Tests and Misdiagnosis of Imported Malaria: Are PCR-Based Reference Laboratories the Answer? Journal of Clinical Microbiology 2002. 40(2): p. 736-737.

10. RW Peeling, K.H., D Mabey, et al., Rapid tests for sexually transmitted infections (STIs): the way forward. STI Journal, 2006: p. 1-6.

11. Ahmedin Jemal, F.B., David Forman, Global Cancer Statistics. CA: A Cancer Journal for Clinicians, 2011. 61: p. 69-90.

12. Kayhan Parsi, D.B., Justin List, The Dread Disease: Cancer in the Developing World. Hastings Center Report, 2011: p. 13-14.

13. R.A. Malkin, Design of Health Care Technologies for the Developing World. Annual Review of Biomedical Engineering, 2007. 9: p. 567-587.

14. D.M. Kemeny, S.J.C., ELISA and Other Solid Phase Immunoassays: Theoretical and Practical Aspects. 1988, Portsmouth: John WIley \& Sons Ltd.

15. S.F. Sun, Souther Blot, Northern Blot, and Western Blot, in Physical Chemistry of Macromolecules: Basic Principles and Issues. 2004, John Wiley \& Sons, Inc.: Jamaica, New York.

16. Raphael C. Wong, H.Y.T., Lateral Flow Immunoassay. 2008, Springer: New York, NY. p. 39.

17. Curtis D. Chin, V.L., Samuel K. Sia, Lab-on-a-chip devices for global health: Past studies and future opportunities. Lab on a Chip, 2006. 7: p. 41-57. 
18. George Whitesides, George Whitesides: A lab the size of a postage stamp, in TEDxBoston, TEDX, Editor. 2009: Boston, MA.

19. Andres W. Martinez, S.T.P., George M. Whitesides, Three-dimensional microfluidic devices fabricated in layered paper and tape. Proceedings of National Academy of Sciences, 2008. 105(50): p. 19606-19611.

20. Andres W. Martinez, S.T.P., Benjamin J. Wiley, Malancha Gupta, George M. Whitesides, FLASH: A rapid method for prototyping paper-based microfluidic devices. Lab on a Chip, 2008. 8: p. 2146-2150.

21. Emanuel Carrilho, A.W.M., George M. Whitesides, Understanding Wax Printing - A Simple Micropatterning Process for Paper-Based Microfluidics. Analytical Chemistry, 2009. Manuscript: p. 1-18.

22. Emanuel Carrilho, S.T.P., Sarah J. Vella, Andres W. Martinez, George M. Whitesides, Paper Microzone Plates. Analytical Chemistry, 2009. Manuscript: p. 1-32.

23. Millipore (2008) Rapid Lateral Flow Test Strips. Volume,

24. Weian Zhao, A.v.d.B., Lab on paper. Lab on a Chip, 2008. 8: p. 1988-1991.

25. Biooncology, G. Glioblastomas, angiogenesis, and VEGF. Volume,

26. Mark L. George, M.G.T., Frank Janssen, Abed Arnaout, A. Muti Abulafi, Suzanne A. Eccles, R. Ian Swift, VEGF-A, VEGF-C, and VEGF-D in Colorectal Cancer Progression. Nature, 2001. 3(5): p. 420-427.

27. J.H. Harmey, VEGF and Cancer. 2004, New York, NY: Kluwer Academic / Plenum Publishers.

28. Cleber A. Trujillo, A.A.N., Janaína M. Alves, Antonio H. Martins, Henning Ulrich, Development of the anti-VEGF aptamer to a therapeutic agent for clinical ophthamology. Clinical Ophthamology, 2007. 1(4): p. 393-402.

29. Yoshihiko Nonaka, K.S., Kazunori Ikebukuro, Screening and Improvement of an Anti-VEGF DNA Aptamer. Molecules, 2010. 15: p. 215-225.

30. Itsuro Endo, T.M., Maki Nishino, Yasushi Oshima, Toshio Matsumoto, Diurnal Fluctuation of Edema Synchronized with Plasma VEGF Concentration in a Patient with POEMS Syndrome. Internal Medicine, 2002. 41(12): p. 11961198.

31. Marina Scarlato, S.C.P., Marinella Carpo, Davide Pareyson, Chiara Briani, Roberto Del Bo, Eduardo Nobile-Orazio, Angelo Quattrini, Giacomo Pietro Comi, Polyneuropathy in POEMS syndrome: role of angiogenic factors in pathogenesis. Brain, 2005. 128: p. 1911-1920.

32. N. Orazio, Serum VEGF levels in POEMS syndrome and in immune-mediated neuropathies. Neurology, 2009. 72(11): p. 1024-1026.

33. Vittorio Rosti, M.M., Rita Campanelli, Mara De Amici, Giovanni Piccolo, Vittorio Perfetti, Vascular endothelial growth factor promoted endothelial progenitor cell mobilization intro the peripheral blood of a patient with POEMS syndrome. The Hematology Journal, 2007. 92(09): p. 1291-1292.

34. B.R. Lynn Sterling, Protocol Development for Ovarian Cancer Treatment in Kenya. International Journal of Gynecological Cancer, 2011. 21(2): p. 424427.

35. Joon-Hwa Lee, M.D.C., Andrea De Erkenez, Dominik Krilleke, Yin-Shan Ng, David T. Shima, Arthur Pardi, Fiona Jucker, A therapeutic aptamer inhibits 
angiogenesis by specifically targeting the heparin binding domain of VEGF165. Proceedings of National Academy of Sciences of the United States of America, 2005. 102(52): p. 18902-18907.

36. Anastasios J. Karayiannakis, K.N.S., Alexandros Polychronidis, Andrew Zbar, Gregory Kouraklis, Constantinos Simopoulos, Gabriel Karatzas, Circulating VEGF Levels in the Serum of Gastric Cancer Patients. Annals of Surgery, 2002. 236(1): p. 37-42.

37. Laurie M. Elit, D.M., Teaching Cervical Cancer Surgery in Low- or MiddleResource Countries. International Journal of Gynecological Cancer, 2010. 20(9): p. 1604-1608.

38. Edward Harlow, D.L., Antibodies: A Laboratory Manual. 1988, Cold Spring Harbor, NY: Cold Spring Harbor Laboratory Press.

39. Juewen Liu, D.M., and Yi Lu, A Simple and Sensitive "Dipstick" Test in Serum Based on Lateral Flow Separation of Aptamer-Linked Nanostructures. Angewandte Chemie International Edition 2006. 45: p. 7955-7959.

40. R.M. L Taniwaki, AS Cunha-Júnior, AAG Faraco, JAS Ribiero, IU Scott, R Jorge, Effect of lyophilization on the in vitro biological activity of bevacizumab. Eye, 2010. 24: p. 1628-1629.

41. Enzyme Linked Immunosorbent Assays (ELISA). [cited 2012 March 10, 2012]; Available from: http://www.geneticasysid.com.br/ELISA.htm.

42. M.M. S. Tombelli, M. Mascini, Analytical applications of aptamers. Biosensors and Bioelectronics, 2004. 20(2005): p. 2424-2434.

43. S.D. Jayasena, Aptamers: An Emerging Class of Molecules That Rival Antibodies in Diagnostics. Clinical Chemistry, 1999. 45(9): p. 1628-1650.

44. Wijitar Dungchai, O.C., Charles S. Henry, Electrochemical Detection for PaperBased Microfluidics. Analytical Chemistry, 2009. 81(14): p. 5821-5826.

45. Yao Lu, W.S., Jianhua Qin, Bingcheng Lin, Fabrication and Characterization of Paper-Based Microfluidics Prepared in Nitrocellulose Membrane By Wax Printing. Analytical Chemistry, 2010. 82(1): p. 329-335.

46. R. Davies, How to attach antibodies on gold. [cited; Available from: https://mail.mems-exchange.org/mems-talk/11091/.

47. Bianmei Cao, T.X., David Hui, Preparation of Nanogold Labeled Goat-AntiRabbit IgG and Their Application In An Immunoassay. Metalurgija - MJoM, 2011. 17(2): p. 79-85.

48. Shuo Wang, C.Z., Junping Wang, Yan Zhang, Development of colloidal goldbased flow-through and lateral-flow immunoassays for the rapid detection of the insecticide carbaryl. Alaytica Chimica, 2005. 546: p. 161-166.

49. Weian Zhao, M.M.A., Sergio D. Aguirre, Michael A. Brook, Yigfu Li, PaperBased Bioassays Using Gold Nanoparticle Colorimetric Probes. Analytical Chemistry, 2008. 80(22): p. 8431-8437.

50. V. Wu, Imminogen sequence of bsm-4572M-Gold, K. Clayton, Editor. 2012, Bioss-USA: Woburn, MA.

51. Health, N.I.o. BLAST: Basic Local Alignment Search Tool. 2012 [cited 2012; Available from: http://blast.ncbi.nlm.nih.gov/.

52. Mary A. Dorman, C.D.B., James K. Collins, Barry J. Beaty, Detection of Bovine Herpesvirus 1 DNA Immobilized on Nitrocellulose by Hybridization with 
Biotinylated DNA Probes. Journal of Clinical Microbiology, 1985. 22(6): $\mathrm{p}$. 990-995.

53. Ajish S.R. Potty, K.K., Han Fang, Peter Schuck, Richard C. Willson, Biophysical characterization of DNA and RNA aptamer interactions with hen egg lysozyme. International Journal of Biological Macromolecules, 2010. 48(2011): p. 392397.

54. Bo Johnsson, S.L., Gabrielle Lindquist, Åsa Endström, Rose-Marie Müller Hillgren, Anna Hansson, Comparison of Methods for Immobilization to Carboxymethyl Dextran Sensor Surfaces by Analysis of the Specific Activity of Monoclonal Antibodies. Journal of Molecular Recognition, 1995. 8: p. 125-131.

55. Alexander Volkov, M.M., Paul Corstjens, R. Sam Niedbala, Rapid Prototyping of Lateral Flow Assays. Methods in Molecular Biology: Biosensors and Biodetection, 2009. 504(3): p. 217-235.

56. M. Mansfield, Millipore and Antibody Discussion, K. Clayton, Editor. 2012, Millipore: Millerica, MA. 


\section{Appendix A: Statistical Analyses for Chip Design Characteristics}

The following tables and statistical output were not using throughout the main report due to their insignificance in describing the conclusions reached for this thesis.

However they are provided here for completeness, for fully understanding the methods

followed, and the information that aided in finding results and conclusions in this project.

\section{Table XIV: First Thirty-Two Tests}

The first thirty-two statistical tests conducted, where treatments/experimental order were assigned at random using Microsoft Excel

\begin{tabular}{|c|c|c|c|c|c|}
\hline Treatment & Backing & Curving & Porosity & Size & Width \\
\hline 1 & Backed & Straight & 75 & Short & Narrow \\
\hline 2 & Unbacked & Curved & 135 & Short & Wide \\
\hline 3 & Backed & Curved & 135 & Short & Narrow \\
\hline 4 & Backed & Curved & 135 & Long & Wide \\
\hline 5 & Unbacked & Straight & 135 & Short & Wide \\
\hline 6 & Unbacked & Curved & 180 & Short & Narrow \\
\hline 7 & Backed & Curved & 75 & Long & Narrow \\
\hline 8 & Backed & Curved & 75 & Long & Wide \\
\hline 9 & Unbacked & Curved & 180 & Long & Wide \\
\hline 10 & Backed & Curved & 135 & Long & Narrow \\
\hline 11 & Backed & Straight & 75 & Long & Wide \\
\hline 12 & Unbacked & Curved & 135 & Long & Narrow \\
\hline 13 & Unbacked & Straight & 180 & Short & Narrow \\
\hline 14 & Unbacked & Curved & 135 & Short & Narrow \\
\hline 15 & Backed & Curved & 75 & Short & Narrow \\
\hline 16 & Backed & Straight & 135 & Long & Wide \\
\hline 17 & Backed & Curved & 135 & Short & Wide \\
\hline 18 & Unbacked & Curved & 180 & Long & Narrow \\
\hline 19 & Backed & Straight & 135 & Short & Wide \\
\hline 20 & Unbacked & Straight & 180 & Long & Narrow \\
\hline 21 & Unbacked & Curved & 135 & Long & Wide \\
\hline 22 & Backed & Straight & 75 & Long & Narrow \\
\hline 23 & Unbacked & Straight & 135 & Long & Narrow \\
\hline 24 & Unbacked & Straight & 180 & Short & Wide \\
\hline 25 & Unbacked & Straight & 135 & Long & Wide \\
\hline 26 & Unbacked & Straight & 135 & Short & Narrow \\
\hline 27 & Backed & Straight & 135 & Short & Narrow \\
\hline 28 & Backed & Straight & 135 & Long & Narrow \\
\hline 29 & Unbacked & Straight & 180 & Long & Wide \\
\hline 30 & Unbacked & Curved & 180 & Short & Wide \\
\hline 31 & Backed & Curved & 75 & Short & Wide \\
\hline 32 & Backed & Straight & 75 & Short & Wide \\
\hline
\end{tabular}




\section{Table XV: Second Thirty-Two Tests}

The second thirty-two statistical tests conducted, where treatments/experimental order were assigned at random using Microsoft Excel

$\begin{array}{cccccc}\text { Treatment } & \text { Backing } & \text { Curving } & \text { Porosity } & \text { Size } & \text { Width } \\ 1 & \text { Unbacked } & \text { Curved } & 180 & \text { Long } & \text { Wide } \\ 2 & \text { Unbacked } & \text { Curved } & 135 & \text { Long } & \text { Narrow } \\ 3 & \text { Backed } & \text { Curved } & 135 & \text { Long } & \text { Narrow } \\ 4 & \text { Unbacked } & \text { Curved } & 180 & \text { Short } & \text { Wide } \\ 5 & \text { Unbacked } & \text { Curved } & 180 & \text { Short } & \text { Narrow } \\ 6 & \text { Unbacked } & \text { Curved } & 135 & \text { Long } & \text { Wide } \\ 7 & \text { Unbacked } & \text { Straight } & 135 & \text { Short } & \text { Narrow } \\ 8 & \text { Unbacked } & \text { Straight } & 135 & \text { Short } & \text { Wide } \\ 9 & \text { Unbacked } & \text { Straight } & 180 & \text { Short } & \text { Wide } \\ 10 & \text { Backed } & \text { Curved } & 135 & \text { Long } & \text { Wide } \\ 11 & \text { Backed } & \text { Straight } & 75 & \text { Long } & \text { Narrow } \\ 12 & \text { Unbacked } & \text { Straight } & 135 & \text { Long } & \text { Narrow } \\ 13 & \text { Backed } & \text { Curved } & 75 & \text { Short } & \text { Wide } \\ 14 & \text { Backed } & \text { Straight } & 75 & \text { Long } & \text { Wide } \\ 15 & \text { Backed } & \text { Straight } & 75 & \text { Short } & \text { Wide } \\ 16 & \text { Backed } & \text { Straight } & 135 & \text { Short } & \text { Narrow } \\ 17 & \text { Unbacked } & \text { Curved } & 135 & \text { Short } & \text { Narrow } \\ 18 & \text { Unbacked } & \text { Straight } & 135 & \text { Long } & \text { Wide } \\ 19 & \text { Unbacked } & \text { Curved } & 180 & \text { Long } & \text { Narrow } \\ 20 & \text { Unbacked } & \text { Straight } & 180 & \text { Long } & \text { Wide } \\ 21 & \text { Backed } & \text { Straight } & 135 & \text { Short } & \text { Wide } \\ 22 & \text { Backed } & \text { Curved } & 75 & \text { Long } & \text { Narrow } \\ 23 & \text { Unbacked } & \text { Curved } & 135 & \text { Short } & \text { Wide } \\ 24 & \text { Backed } & \text { Curved } & 75 & \text { Short } & \text { Narrow } \\ 25 & \text { Backed } & \text { Curved } & 75 & \text { Long } & \text { Wide } \\ 26 & \text { Backed } & \text { Straight } & 135 & \text { Long } & \text { Wide } \\ 27 & \text { Unbacked } & \text { Straight } & 180 & \text { Short } & \text { Narrow } \\ 28 & \text { Unbacked } & \text { Straight } & 180 & \text { Long } & \text { Narrow } \\ 29 & \text { Backed } & \text { Straight } & 75 & \text { Short } & \text { Narrow } \\ 30 & \text { Backed } & \text { Curved } & 135 & \text { Short } & \text { Narrow } \\ 31 & \text { Backed } & \text { Straight } & 135 & \text { Long } & \text { Narrow } \\ 32 & \text { Backed } & \text { Curved } & 135 & \text { Short } & \text { Wide }\end{array}$


Here is the Minitab output comparing all interactions possible regarding the backing, curvature, porosity, lengths, and widths of designs on nitrocellulose membrane in regard to wicking time.

\begin{tabular}{|c|c|c|c|c|c|}
\hline Source & $\mathrm{DF}$ & Seq SS & Adj $S S$ & Adj MS & 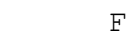 \\
\hline Backing & 1 & 3.92230 & 3.92230 & 3.92230 & 110.98 \\
\hline Curving & 1 & 1.46350 & 1.46350 & 1.46350 & 41.41 \\
\hline Porosity & 1 & 5.43164 & 5.43164 & 5.43164 & 153.69 \\
\hline Size & 1 & 3.63407 & 3.63407 & 3.63407 & 102.83 \\
\hline Width & 1 & 0.12649 & 0.12649 & 0.12649 & 3.58 \\
\hline Backing*Curving & 1 & 0.01984 & 0.01984 & 0.01984 & 0.56 \\
\hline Backing*Porosity & 1 & 0.18685 & 0.18685 & 0.18685 & 5.29 \\
\hline Backing*Size & 1 & 0.88287 & 0.88287 & 0.88287 & 24.98 \\
\hline Backing*Width & 1 & 0.28513 & 0.28513 & 0.28513 & 8.07 \\
\hline Curving*Porosity & 1 & 0.00759 & 0.00759 & 0.00759 & 0.21 \\
\hline Curving*Size & 1 & 0.02229 & 0.02229 & 0.02229 & 0.63 \\
\hline Curving*Width & 1 & 0.13143 & 0.13143 & 0.13143 & 3.72 \\
\hline Porosity*Size & 1 & 0.38576 & 0.38576 & 0.38576 & 10.92 \\
\hline Porosity*Width & 1 & 0.34372 & 0.34372 & 0.34372 & 9.73 \\
\hline Size*Width & 1 & 0.01934 & 0.01934 & 0.01934 & 0.55 \\
\hline Backing*Curving*Porosity & 1 & 0.14515 & 0.14515 & 0.14515 & 4.11 \\
\hline Backing*Curving*Size & 1 & 0.17992 & 0.17992 & 0.17992 & 5.09 \\
\hline Backing*Curving*Width & 1 & 0.06833 & 0.06833 & 0.06833 & 1.93 \\
\hline Backing*Porosity*Size & 1 & 0.12423 & 0.12423 & 0.12423 & 3.52 \\
\hline Backing*Porosity*Width & 1 & 0.10047 & 0.10047 & 0.10047 & 2.84 \\
\hline Backing*Size*Width & 1 & 0.41725 & 0.41725 & 0.41725 & 11.81 \\
\hline Curving*Porosity*Size & 1 & 0.20512 & 0.20512 & 0.20512 & 5.80 \\
\hline Curving*Porosity*Width & 1 & 0.15846 & 0.15846 & 0.15846 & 4.48 \\
\hline Curving*Size*Width & 1 & 0.00019 & 0.00019 & 0.00019 & 0.01 \\
\hline Porosity*Size*Width & 1 & 0.34707 & 0.34707 & 0.34707 & 9.82 \\
\hline Backing*Curving*Porosity*Size & 1 & 0.09133 & 0.09133 & 0.09133 & 2.58 \\
\hline Backing*Curving*Porosity*Width & 1 & 0.45561 & 0.45561 & 0.45561 & 12.89 \\
\hline Backing*Curving*Size*Width & 1 & 0.01903 & 0.01903 & 0.01903 & 0.54 \\
\hline Backing*Porosity*Size*Width & 1 & 0.12568 & 0.12568 & 0.12568 & 3.56 \\
\hline Curving*Porosity*Size*Width & 1 & 0.05602 & 0.05602 & 0.05602 & 1.58 \\
\hline Backing*Curving*Porosity*Size*Width & 1 & 0.00083 & 0.00083 & 0.00083 & 0.02 \\
\hline Error & 32 & 1.13094 & 1.13094 & 0.03534 & \\
\hline Total & 63 & 20.48842 & & & \\
\hline Source & & $\mathrm{P}$ & & & \\
\hline Backing & 0.0 & & & & \\
\hline Curving & 0.0 & & & & \\
\hline Porosity & 0.0 & & & & \\
\hline Size & 0.0 & & & & \\
\hline Width & 0.0 & & & & \\
\hline Backing*Curving & 0.4 & & & & \\
\hline Backing*Porosity & 0.0 & & & & \\
\hline Backing*Size & 0.0 & & & & \\
\hline Backing*Width & 0.0 & & & & \\
\hline Curving*Porosity & 0.6 & & & & \\
\hline Curving*Size & 0.4 & & & & \\
\hline Curving*Width & 0.0 & & & & \\
\hline Porosity*Size & 0.0 & & & & \\
\hline Porosity*Width & 0.0 & & & & \\
\hline Size*Width & 0.4 & & & & \\
\hline Backing*Curving*Porosity & 0.0 & & & & \\
\hline
\end{tabular}


Backing*Curving*Size

Backing*Curving*Width

Backing*Porosity*Size

Backing*Porosity*Width

Backing*Size*Width

Curving*Porosity*Size

Curving*Porosity*Width

Curving*Size*Width

Porosity*Size*Width

Backing*Curving*Porosity*Size

Backing Curving * Porosity*Width

Backing ${ }^{*}$ Curving*Size*Width

Backing*Porosity*Size*Width

Curving*Porosity*Size*Width

Backing*Curving*Porosity*Size*Width

Error

Total
0.031

0.174

0.070

0.102

0.002

0.022

0.042

0.943

0.004

0.118

0.001

0.468

0.068

0.217

0.879 
ANOVA Output for the Initial Model

\section{General Linear Model: Time versus Backing, Curving, ...}

$\begin{array}{llrl}\text { Factor } & \text { Type } & \text { Levels } & \text { Values } \\ \text { Backing } & \text { fixed } & 2 & \text { Backed, Unbacked } \\ \text { Curving } & \text { fixed } & 2 & \text { Curved, Straight } \\ \text { Porosity } & \text { fixed } & 2 & \text { hi, lo } \\ \text { Size } & \text { fixed } & 2 & \text { Long, Short } \\ \text { Width } & \text { fixed } & 2 & \text { Narrow, Wide }\end{array}$

Analysis of Variance for Time, using Adjusted SS for Tests

\begin{tabular}{|c|c|c|c|c|c|c|}
\hline Source & $\mathrm{DF}$ & Seq SS & Adj SS & Adj MS & $\mathrm{F}$ & 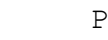 \\
\hline Backing & 1 & 264440 & 264440 & 264440 & 411.74 & 0.000 \\
\hline Curving & 1 & 154046 & 154046 & 154046 & 239.86 & 0.000 \\
\hline Porosity & 1 & 305933 & 305933 & 305933 & 476.35 & 0.000 \\
\hline Size & 1 & 1512 & 1512 & 1512 & 2.35 & 0.135 \\
\hline idth & 1 & 66596 & 66596 & 66596 & 103.69 & 0.000 \\
\hline acking*Curving & 1 & 89125 & 89125 & 89125 & 138.77 & 0.000 \\
\hline acking*Porosity & 1 & 181189 & 181189 & 181189 & 282.12 & 0.000 \\
\hline acking*Size & 1 & 57091 & 57091 & 57091 & 88.89 & 0.000 \\
\hline acking*Width & 1 & 81375 & 81375 & 81375 & 126.70 & 0.000 \\
\hline urving*Porosity & 1 & 95581 & 95581 & 95581 & 148.82 & 0.000 \\
\hline Curving*Size & 1 & 58582 & 58582 & 58582 & 91.21 & 0.000 \\
\hline Curving*Width & 1 & 122579 & 122579 & 122579 & 190.86 & 0.000 \\
\hline Porosity*Size & 1 & 39526 & 39526 & 39526 & 61.54 & 0.000 \\
\hline Porosity*Width & 1 & 81560 & 1560 & 81560 & 126.99 & 0.000 \\
\hline Size*Width & 1 & 77960 & 7960 & 77960 & 121.39 & 0.000 \\
\hline acking*Curving*Porosity & 1 & 86238 & 36238 & 86238 & 134.28 & 0.000 \\
\hline Backing*Curving*Size & 1 & 97992 & 97992 & 97992 & 152.58 & 0.000 \\
\hline acking*Curving*Width & 1 & 128854 & 128854 & 128854 & 200.63 & 0.000 \\
\hline acking*Porosity*Size & 1 & 73123 & 73123 & 73123 & 113.86 & 0.000 \\
\hline Backing*Porosity ${ }^{*}$ Width & 1 & 87283 & 87283 & 87283 & 135.90 & 0.000 \\
\hline Backing*Size*Width & 1 & 87594 & 87594 & 87594 & 136.39 & 0.000 \\
\hline Curving*Porosity*Size & 1 & 90518 & 90518 & 90518 & 140.94 & 0.000 \\
\hline Curving*Porosity*Width & 1 & 147101 & 147101 & 147101 & 229.04 & 0.000 \\
\hline Curving*Size*Width & 1 & 3092 & 30928 & 30928 & 48.16 & 0.000 \\
\hline Porosity*Size*Width & 1 & 854 & 85432 & 85432 & 133.02 & 0.000 \\
\hline Backing*Curving*Porosity*Size & 1 & 910 & 91091 & 91091 & 141.83 & 0.000 \\
\hline Backing*Curving*Porosity*Width & 1 & 1543 & 154321 & 154321 & 240.28 & 0.000 \\
\hline Backing*Curving*Size*Width & 1 & 31351 & 31351 & 31351 & 48.81 & 0.000 \\
\hline Backing*Porosity*Size*Width & 1 & 88202 & 88202 & 88202 & 137.33 & 0.000 \\
\hline Curving*Porosity*Size*Width & 1 & 2354 & 2354 & 23543 & 36.66 & 0.000 \\
\hline Backing*Curving*Porosity*Size*Width & 1 & 257 & 2572 & 25724 & 40.05 & 0.000 \\
\hline rror & 32 & 205 & 20552 & 642 & & \\
\hline & 63 & & & & & \\
\hline
\end{tabular}

$S=25.3426 \quad R-S q=99.32 \% \quad R-S q(\operatorname{adj})=98.66 \%$

Unusual Observations for Time

$\begin{array}{rrrrrr}\text { Obs } & \text { Time } & \text { Fit } & \text { SE Fit } & \text { Residual } & \text { St Resid } \\ 9 & 357.50 & 311.30 & 17.92 & 46.20 & 2.58 \mathrm{R} \\ 13 & 97.40 & 60.20 & 17.92 & 37.20 & 2.08 \mathrm{R} \\ 25 & 20.00 & 62.95 & 17.92 & -42.95 & -2.40 \mathrm{R} \\ 30 & 1200.00 & 1245.30 & 17.92 & -45.30 & -2.53 \mathrm{R} \\ 33 & 265.10 & 311.30 & 17.92 & -46.20 & -2.58 \mathrm{R} \\ 36 & 1290.60 & 1245.30 & 17.92 & 45.30 & 2.53 \mathrm{R}\end{array}$




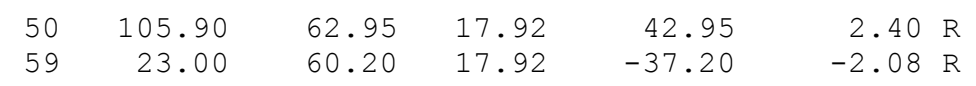

$\mathrm{R}$ denotes an observation with a large standardized residual. 
The following values are for the Cook's distances for the initial statistical model.

\section{COOK4}

$\begin{array}{ll}0.000002 & 0.016967 \\ 0.076924 & 0.004872 \\ 0.020970 & 0.000442 \\ 0.033003 & 0.004520 \\ 0.057922 & 0.070392 \\ 0.004520 & 0.000009 \\ 0.000006 & 0.057922 \\ 0.001996 & 0.001515 \\ 0.007456 & 0.033003 \\ 0.004872 & 0.000517 \\ 0.000085 & 0.026726 \\ 0.016967 & 0.005063 \\ 0.173713 & 0.000085 \\ 0.000246 & 0.000331 \\ 0.008772 & 0.054164 \\ 0.052204 & 0.000246 \\ 0.092205 & 0.231658 \\ 0.000601 & 0.000601 \\ 0.040064 & 0.014258 \\ 0.000454 & 0.040064 \\ 0.070392 & 0.000006 \\ 0.000517 & 0.076924 \\ 0.026726 & 0.008772 \\ 0.001515 & 0.001996 \\ 0.231658 & 0.052204 \\ 0.000009 & 0.173713 \\ 0.054164 & 0.000454 \\ 0.001944 & 0.000002 \\ 0.014258 & 0.020970 \\ 0.000442 & 0.001944 \\ 0.005063 & 0.092205 \\ 0.000331 & \\ 0.007456 & \\ 0 & \\ 0 & \\ 0 & \end{array}$


ANOVA Output for the Transformed $\log ()$ Model

\section{General Linear Model: log(time) versus Backing, Curving, ...}

$\begin{array}{llrl}\text { Factor } & \text { Type } & \text { Levels } & \text { Values } \\ \text { Backing } & \text { fixed } & 2 & \text { Backed, Unbacked } \\ \text { Curving } & \text { fixed } & 2 & \text { Curved, Straight } \\ \text { Porosity } & \text { fixed } & 2 & \text { hi, lo } \\ \text { Size } & \text { fixed } & 2 & \text { Long, Short } \\ \text { Width } & \text { fixed } & 2 & \text { Narrow, Wide }\end{array}$

Analysis of Variance for log(time), using Adjusted ss for Tests

\begin{tabular}{|c|c|c|c|c|c|}
\hline Source & $\mathrm{DF}$ & Seq SS & Adj SS & Adj MS & 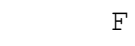 \\
\hline Backing & 1 & 3.92230 & 3.92230 & 3.92230 & 110.98 \\
\hline Curving & 1 & 1.46350 & 1.46350 & 1.46350 & 41.41 \\
\hline Porosity & 1 & 5.43164 & 5.43164 & 5.43164 & 153.69 \\
\hline Size & 1 & 3.63407 & 3.63407 & 3.63407 & 102.83 \\
\hline Width & 1 & 0.12649 & 0.12649 & 0.12649 & 3.58 \\
\hline Backing*Curving & 1 & 0.01984 & 0.01984 & 0.01984 & 0.56 \\
\hline Backing*Porosity & 1 & 0.18685 & 0.18685 & 0.18685 & 5.29 \\
\hline Backing*Size & 1 & 0.88287 & 0.88287 & 0.88287 & 24.98 \\
\hline Backing*Width & 1 & 0.28513 & 0.28513 & 0.28513 & 8.07 \\
\hline Curving*Porosity & 1 & 0.00759 & 0.00759 & 0.00759 & 0.21 \\
\hline Curving*Size & 1 & 0.02229 & 0.02229 & 0.02229 & 0.63 \\
\hline Curving*Width & 1 & 0.13143 & 0.13143 & 0.13143 & 3.72 \\
\hline Porosity*Size & 1 & 0.38576 & 0.38576 & 0.38576 & 10.92 \\
\hline Porosity*Width & 1 & 0.34372 & 0.34372 & 0.34372 & 9.73 \\
\hline Size*Width & 1 & 0.01934 & 0.01934 & 0.01934 & 0.55 \\
\hline Backing*Curving*Porosity & 1 & 0.14515 & 0.14515 & 0.14515 & 4.11 \\
\hline Backing*Curving*Size & 1 & 0.17992 & 0.17992 & 0.17992 & 5.09 \\
\hline Backing*Curving*Width & 1 & 0.06833 & 0.06833 & 0.06833 & 1.93 \\
\hline Backing*Porosity*Size & 1 & 0.12423 & 0.12423 & 0.12423 & 3.52 \\
\hline Backing*Porosity*Width & 1 & 0.10047 & 0.10047 & 0.10047 & 2.84 \\
\hline Backing*Size*Width & 1 & 0.41725 & 0.41725 & 0.41725 & 11.81 \\
\hline Curving*Porosity*Size & 1 & 0.20512 & 0.20512 & 0.20512 & 5.80 \\
\hline Curving*Porosity*Width & 1 & 0.15846 & 0.15846 & 0.15846 & 4.48 \\
\hline Curving*Size*Width & 1 & 0.00019 & 0.00019 & 0.00019 & 0.01 \\
\hline Porosity*Size*Width & 1 & 0.34707 & 0.34707 & 0.34707 & 9.82 \\
\hline Backing*Curving*Porosity ${ }^{\star}$ Size & 1 & 0.09133 & 0.09133 & 0.09133 & 2.58 \\
\hline Backing*Curving*Porosity*Width & 1 & 0.45561 & 0.45561 & 0.45561 & 12.89 \\
\hline Backing*Curving*Size*Width & 1 & 0.01903 & 0.01903 & 0.01903 & 0.54 \\
\hline Backing*Porosity*Size*Width & 1 & 0.12568 & 0.12568 & 0.12568 & 3.56 \\
\hline Curving*Porosity*Size*Width & 1 & 0.05602 & 0.05602 & 0.05602 & 1.58 \\
\hline Backing*Curving*Porosity*Size*Width & 1 & 0.00083 & 0.00083 & 0.00083 & 0.02 \\
\hline Error & 32 & 1.13094 & 1.13094 & 0.03534 & \\
\hline Total & 63 & 20.48842 & & & \\
\hline Source & & $\mathrm{P}$ & & & \\
\hline Backing & 0. & & & & \\
\hline Curving & 0. & & & & \\
\hline Porosity & 0. & & & & \\
\hline Size & 0. & & & & \\
\hline Width & 0. & & & & \\
\hline Backing*Curving & 0.4 & & & & \\
\hline Backing*Porosity & 0. & & & & \\
\hline Backing*Size & 0. & & & & \\
\hline Backing*Width & 0. & & & & \\
\hline Curving*Porosity & 0.6 & & & & \\
\hline Curving*Size & 0.4 & & & & \\
\hline$n g *$ Width & 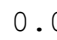 & & & & \\
\hline
\end{tabular}




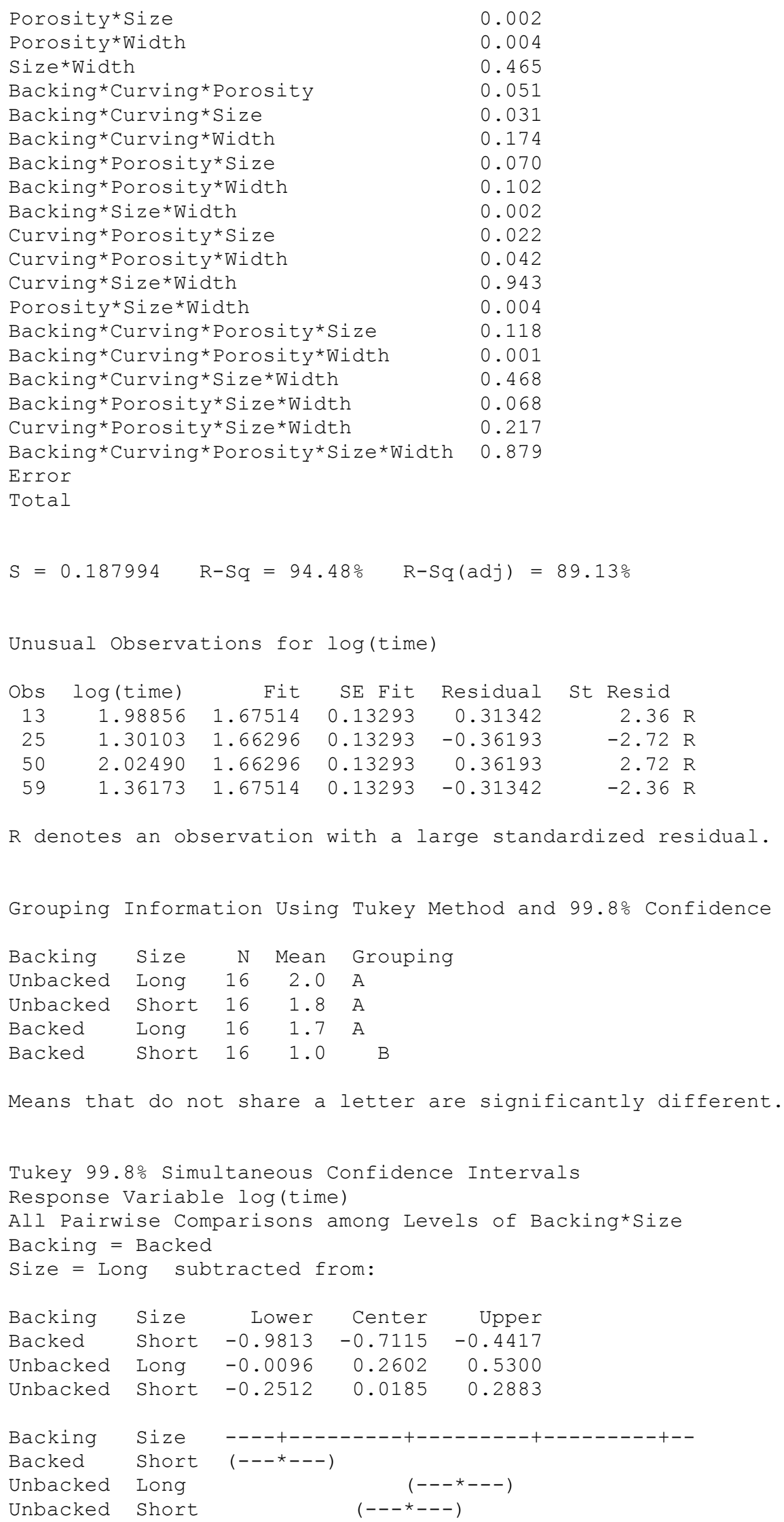



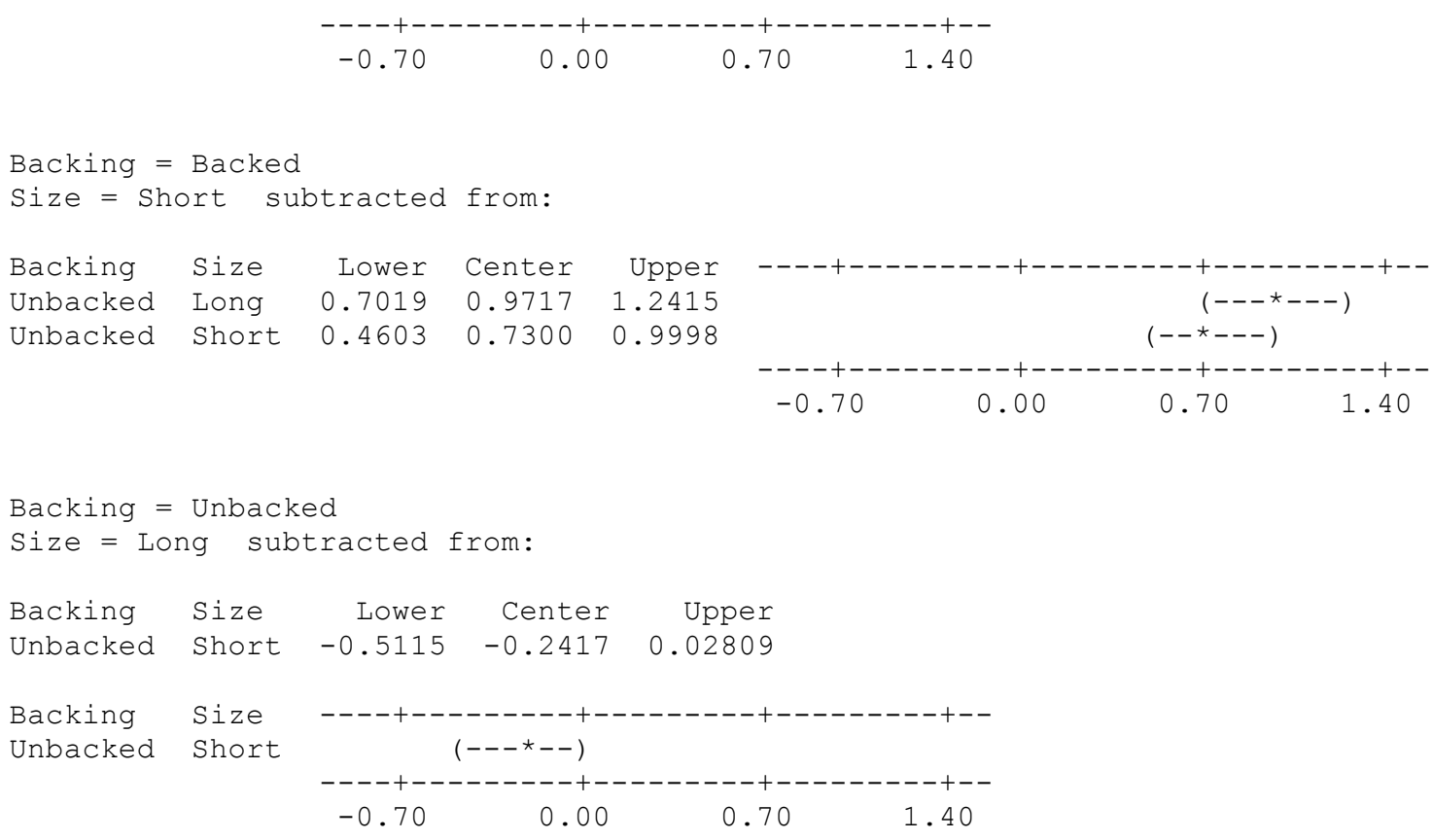

$\begin{array}{llll}-0.70 & 0.00 & 0.70 & 1.40\end{array}$

Tukey Simultaneous Tests

Response Variable log(time)

All Pairwise Comparisons among Levels of Backing*Size Backing = Backed

Size = Long subtracted from:

\begin{tabular}{|c|c|c|c|c|c|}
\hline Backing & Size & $\begin{array}{l}\text { Difference } \\
\text { of Means }\end{array}$ & $\begin{array}{r}\text { SE of } \\
\text { Difference }\end{array}$ & T-Value & $\begin{array}{l}\text { Adjusted } \\
\text { P-Value }\end{array}$ \\
\hline Backed & Short & -0.7115 & 0.06647 & -10.70 & 0.0000 \\
\hline Jnbacked & Long & 0.2602 & 0.06647 & 3.92 & 0.0024 \\
\hline Jnbacked & Short & 0.0185 & 0.06647 & 0.28 & 0.9923 \\
\hline \multicolumn{6}{|c|}{ Backing = Backed } \\
\hline \multicolumn{6}{|c|}{ Size = Short subtracted from: } \\
\hline & & Difference & SE of & & Adjusted \\
\hline acking & Size & of Means & Difference & T-Value & P-Value \\
\hline nbacked & Long & 0.9717 & 0.06647 & 14.62 & 0.0000 \\
\hline nbacked & Short & 0.7300 & 0.06647 & 10.98 & 0.0000 \\
\hline \multicolumn{6}{|c|}{ Backing = Unbacked } \\
\hline \multicolumn{6}{|c|}{ Size = Long subtracted from: } \\
\hline & & Difference & $S E$ of & & Adjusted \\
\hline king & Size & of Means & Difference & T-Value & P-Value \\
\hline nbacked & Short & -0.2417 & 0.06647 & -3.636 & 0.0051 \\
\hline
\end{tabular}

Grouping Information Using Tukey Method and 99.8\% Confidence

$\begin{array}{llllrrrr}\text { Backing } & \text { Curving } & \text { Porosity } & \text { Width } & \text { N } & \text { Mean } & \text { Grouping } \\ \text { Unbacked } & \text { Curved } & \text { hi } & \text { Wide } & 4 & 2.8 & \text { A } & \\ \text { Unbacked } & \text { Curved } & \text { hi } & \text { Narrow } & 4 & 2.1 & \text { B } & \\ \text { Unbacked } & \text { Straight } & \text { hi } & \text { Narrow } & 4 & 2.1 & \text { B C } \\ \text { Unbacked } & \text { Straight } & \text { hi } & \text { Wide } & 4 & 1.9 & \text { B C D }\end{array}$




$\begin{array}{llllllllll}\text { Backed } & \text { Curved } & \text { hi } & \text { Narrow } & 4 & 1.8 & \text { B C D E } & \\ \text { Unbacked } & \text { Curved } & \text { lo } & \text { Narrow } & 4 & 1.8 & \text { B C D E } & \\ \text { Backed } & \text { Curved } & \text { hi } & \text { Wide } & 4 & 1.6 & \text { B C D E } & \\ \text { Backed } & \text { Straight } & \text { hi } & \text { Narrow } & 4 & 1.6 & \text { B C D E } & \\ \text { Unbacked } & \text { Curved } & \text { lo } & \text { Wide } & 4 & 1.5 & \text { B C D E } & \\ \text { Backed } & \text { Straight } & \text { hi } & \text { Wide } & 4 & 1.5 & \text { B C D E } & \\ \text { Unbacked } & \text { Straight } & \text { lo } & \text { Narrow } & 4 & 1.5 & \text { B C D E } & \\ \text { Backed } & \text { Curved } & \text { lo } & \text { Narrow } & 4 & 1.4 & \text { C D E F } \\ \text { Unbacked } & \text { Straight } & \text { lo } & \text { Wide } & 4 & 1.4 & & \text { D E F } \\ \text { Backed } & \text { Curved } & \text { lo } & \text { Wide } & 4 & 1.2 & & \text { E F } \\ \text { Backed } & \text { Straight } & \text { lo } & \text { Narrow } & 4 & 1.2 & & & \text { E F } \\ \text { Backed } & \text { Straight } & \text { lo } & \text { Wide } & 4 & 0.8 & & & & \text { F } \\ \text { Means that do not share a letter are significantly different. }\end{array}$

Tukey 99.8\% Simultaneous Confidence Intervals

Response Variable log(time)

All Pairwise Comparisons among Levels of Backing*Curving*Porosity*Width

Backing $=$ Backed

Curving $=$ Curved

Porosity $=$ hi

Width = Narrow subtracted from:

\begin{tabular}{|c|c|c|c|c|c|c|}
\hline Backing & Curving & Porosity & Width & Lower & Center & Upper \\
\hline Backed & Curved & hi & Wide & -0.869 & -0.199 & 0.4712 \\
\hline Backed & Curved & 10 & Narrow & -1.073 & -0.403 & 0.2672 \\
\hline Backed & Curved & 10 & Wide & -1.268 & -0.598 & 0.0720 \\
\hline Backed & Straight & hi & Narrow & -0.908 & -0.238 & 0.4325 \\
\hline Backed & Straight & hi & Wide & -1.019 & -0.349 & 0.3214 \\
\hline Backed & Straight & 10 & Narrow & -1.319 & -0.649 & 0.0210 \\
\hline Backed & Straight & 10 & Wide & -1.704 & -1.033 & -0.3632 \\
\hline Unbacked & Curved & hi & Narrow & -0.383 & 0.287 & 0.9570 \\
\hline Unbacked & Curved & hi & Wide & 0.311 & 0.981 & 1.6517 \\
\hline Unbacked & Curved & 10 & Narrow & -0.697 & -0.027 & 0.6433 \\
\hline Unbacked & Curved & 10 & Wide & -0.991 & -0.320 & 0.3498 \\
\hline Unbacked & Straight & hi & Narrow & -0.414 & 0.256 & 0.9266 \\
\hline Unbacked & Straight & hi & Wide & -0.568 & 0.103 & 0.7727 \\
\hline Unbacked & Straight & 10 & Narrow & -1.030 & -0.360 & 0.3104 \\
\hline Unbacked & Straight & 10 & Wide & -1.099 & -0.429 & 0.2415 \\
\hline Backing & Curving & Porosity & Width & \multicolumn{3}{|c|}{----+---------+---------+---------+--} \\
\hline Backed & Curved & hi & Wide & \multicolumn{3}{|c|}{$(----\star---)$} \\
\hline Backed & Curved & 10 & Narrow & \multicolumn{3}{|c|}{$(---\star----)$} \\
\hline Backed & Curved & 10 & Wide & \multicolumn{3}{|c|}{$(---\star---)$} \\
\hline Backed & Straight & hi & Narrow & \multicolumn{3}{|c|}{$(---\star----)$} \\
\hline Backed & Straight & hi & Wide & \multicolumn{3}{|c|}{$(----\star---)$} \\
\hline Backed & Straight & 10 & Narrow & \multicolumn{3}{|c|}{$(----\star---)$} \\
\hline Backed & Straight & 10 & Wide & \multicolumn{3}{|c|}{$(---\star----)$} \\
\hline Unbacked & Curved & hi & Narrow & \multicolumn{3}{|c|}{$(----\star---)$} \\
\hline Unbacked & Curved & $\mathrm{hi}$ & Wide & \multicolumn{3}{|c|}{$(----\star---)$} \\
\hline Unbacked & Curved & 10 & Narrow & \multicolumn{3}{|c|}{$(----\star---)$} \\
\hline Unbacked & Curved & 10 & Wide & \multicolumn{3}{|c|}{$(----\star---)$} \\
\hline Unbacked & Straight & hi & Narrow & \multicolumn{3}{|c|}{$(----\star---)$} \\
\hline Unbacked & Straight & hi & Wide & \multicolumn{3}{|c|}{$(----\star---)$} \\
\hline Unbacked & Straight & 10 & Narrow & \multicolumn{3}{|c|}{$(----\star---)$} \\
\hline Unbacked & Straight & 10 & Wide & \multicolumn{3}{|c|}{$(---\star----)$} \\
\hline & & & & \multicolumn{3}{|c|}{ 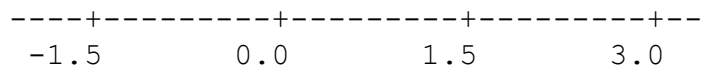 } \\
\hline
\end{tabular}

Backing $=$ Backed

Curving $=$ Curved 


\begin{tabular}{|c|c|c|c|c|c|c|c|}
\hline \multicolumn{8}{|c|}{$\begin{array}{l}\text { Porosity }=\text { hi } \\
\text { Width = wide subtracted from: }\end{array}$} \\
\hline Backing & Curving & Porosity & Width & Lower & Center & \multicolumn{2}{|l|}{ Upper } \\
\hline Backed & Curved & 10 & Narrow & -0.874 & -0.2040 & 0.4662 & \\
\hline Backed & Curved & 10 & Wide & -1.069 & -0.3992 & 0.2710 & \\
\hline Backed & Straight & hi & Narrow & -0.709 & -0.0387 & 0.6315 & \\
\hline Backed & Straight & hi & Wide & -0.820 & -0.1498 & 0.5204 & \\
\hline Backed & Straight & 10 & Narrow & -1.120 & -0.4502 & 0.2200 & \\
\hline Backed & Straight & 10 & Wide & -1.505 & -0.8344 & -0.1642 & \\
\hline Unbacked & Curved & hi & Narrow & -0.184 & 0.4858 & 1.1560 & \\
\hline Unbacked & Curved & hi & Wide & 0.510 & 1.1805 & 1.8507 & \\
\hline Unbacked & Curved & 10 & Narrow & -0.498 & 0.1722 & 0.8424 & \\
\hline Unbacked & Curved & 10 & Wide & -0.792 & -0.1213 & 0.5489 & \\
\hline Unbacked & Straight & hi & Narrow & -0.215 & 0.4554 & 1.1256 & \\
\hline Unbacked & Straight & hi & Wide & -0.369 & 0.3016 & 0.9718 & \\
\hline Unbacked & Straight & 10 & Narrow & -0.831 & -0.1607 & 0.5095 & \\
\hline Unbacked & Straight & 10 & Wide & -0.900 & -0.2297 & 0.4405 & \\
\hline Backing & Curving & Porosity & Width & & ------+- & & \\
\hline Backed & Curved & 10 & Narrow & & $(----\star--$ & & \\
\hline Backed & Curved & 10 & Wide & & $(---\star----$ & & \\
\hline Backed & Straight & hi & Narrow & & $(----*$ & $--)$ & \\
\hline Backed & Straight & hi & Wide & & $(---\star--$ & & \\
\hline Backed & Straight & 10 & Narrow & & $(---\star---)$ & & \\
\hline Backed & Straight & 10 & Wide & $(--$ & $-\star----)$ & & \\
\hline Unbacked & Curved & hi & Narrow & & $(--$ & $-\star----)$ & \\
\hline Unbacked & Curved & hi & Wide & & & $(----\star---)$ & \\
\hline Unbacked & Curved & 10 & Narrow & & $(---\star$ & $----)$ & \\
\hline Unbacked & Curved & 10 & Wide & & $(---\star--1$ & $--)$ & \\
\hline Unbacked & Straight & hi & Narrow & & $(--$ & $-*----)$ & \\
\hline Unbacked & Straight & hi & Wide & & $(--1)$ & $\star---)$ & \\
\hline Unbacked & Straight & 10 & Narrow & & $(----\star--$ & & \\
\hline \multirow[t]{2}{*}{ Unbacked } & \multirow[t]{2}{*}{ Straight } & \multirow[t]{2}{*}{10} & \multirow[t]{2}{*}{ Wide } & \multicolumn{4}{|c|}{$(---\star----)$} \\
\hline & & & & $\begin{array}{c}----+- \\
-1.5\end{array}$ & $\begin{array}{r}0.0 \\
0.0\end{array}$ & 1.5 & 3.0 \\
\hline \\
\hline \multicolumn{8}{|c|}{ Curving = Curved } \\
\hline \multicolumn{8}{|c|}{ Porosity $=10$} \\
\hline \multicolumn{8}{|c|}{ Width = Narrow subtracted from: } \\
\hline Backing & Curving & Porosity & Width & Lower & Center & Upper & \\
\hline Backed & Curved & 10 & Wide & -0.865 & -0.1952 & 0.47498 & \\
\hline Backed & Straight & hi & Narrow & -0.505 & 0.1653 & 0.83552 & \\
\hline Backed & Straight & hi & Wide & -0.616 & 0.0542 & 0.72438 & \\
\hline Backed & Straight & 10 & Narrow & -0.916 & -0.2462 & 0.42403 & \\
\hline Backed & Straight & 10 & Wide & -1.301 & -0.6304 & 0.03978 & \\
\hline Unbacked & Curved & hi & Narrow & 0.020 & 0.6898 & 1.36004 & \\
\hline Unbacked & Curved & hi & Wide & 0.714 & 1.3845 & 2.05467 & \\
\hline Unbacked & Curved & 10 & Narrow & -0.294 & 0.3762 & 1.04637 & \\
\hline Unbacked & Curved & 10 & Wide & -0.588 & 0.0827 & 0.75286 & \\
\hline Unbacked & straight & hi & Narrow & -0.011 & 0.6594 & 1.32960 & \\
\hline Unbacked & Straight & hi & Wide & -0.165 & 0.5055 & 1.17575 & \\
\hline Unbacked & straight & 10 & Narrow & -0.627 & 0.0433 & 0.71346 & \\
\hline Unbacked & Straight & 10 & Wide & -0.696 & -0.0257 & 0.64452 & \\
\hline Backing & Curving & Porosity & Width & $---1+-1$ & ------+ & --------- & \\
\hline Backed & Curved & 10 & Wide & & $(----\star--$ & & \\
\hline Backed & Straight & hi & Narrow & & $(---*$ & $----)$ & \\
\hline Backed & Straight & hi & Wide & & $(---\star-$ & $---)$ & \\
\hline Backed & Straight & 10 & Narrow & & $(---\star---$ & & \\
\hline
\end{tabular}




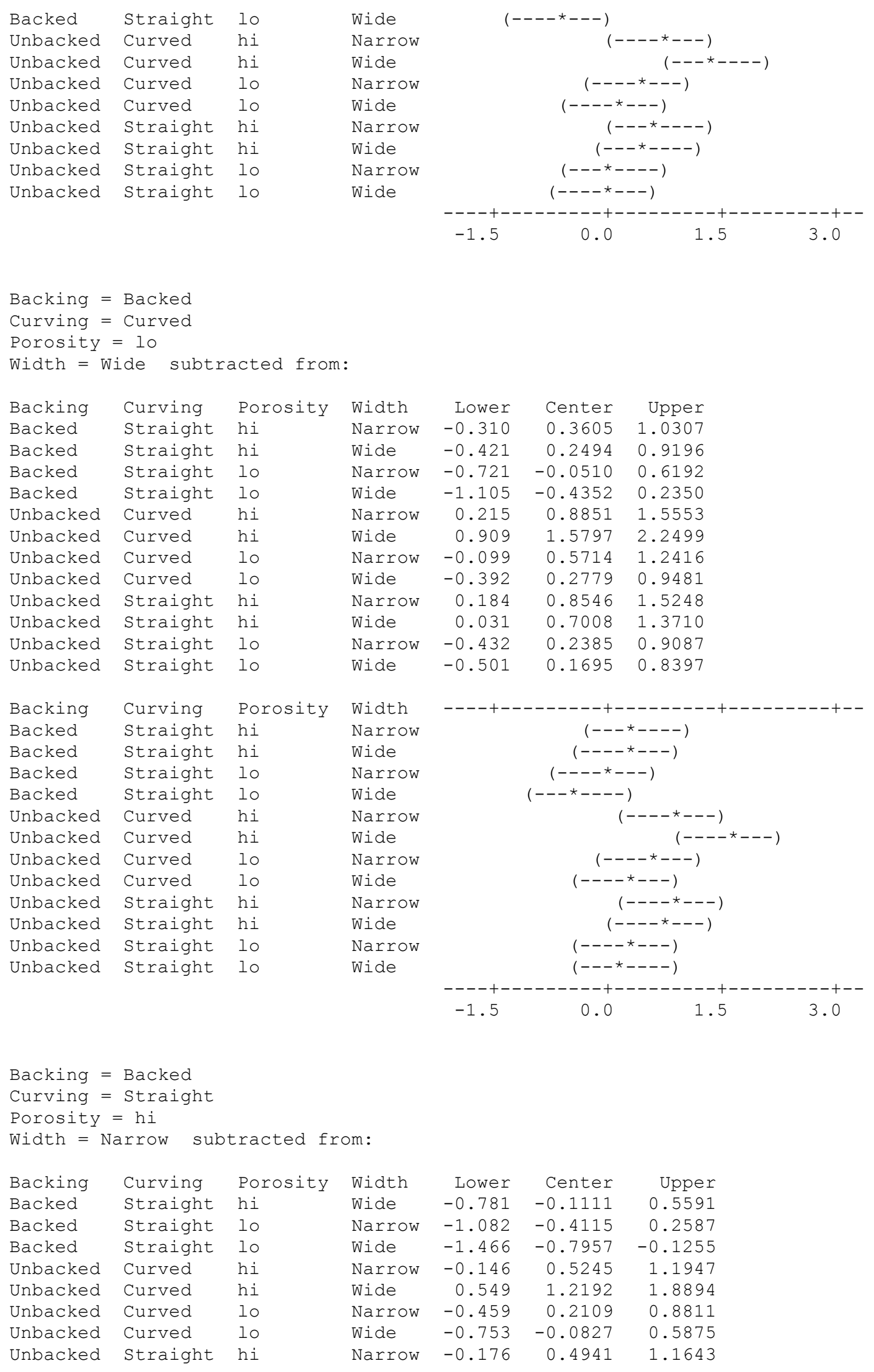




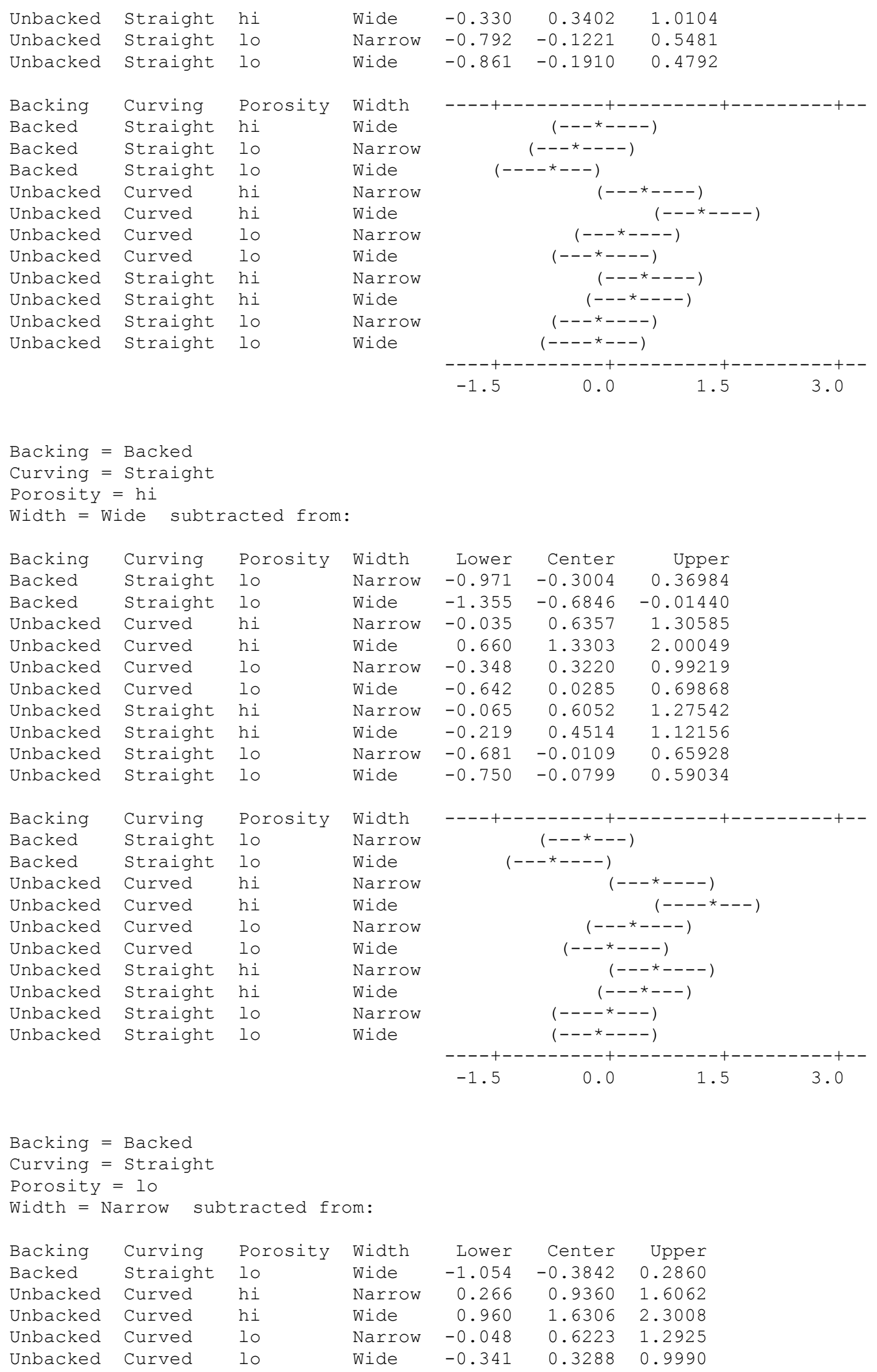




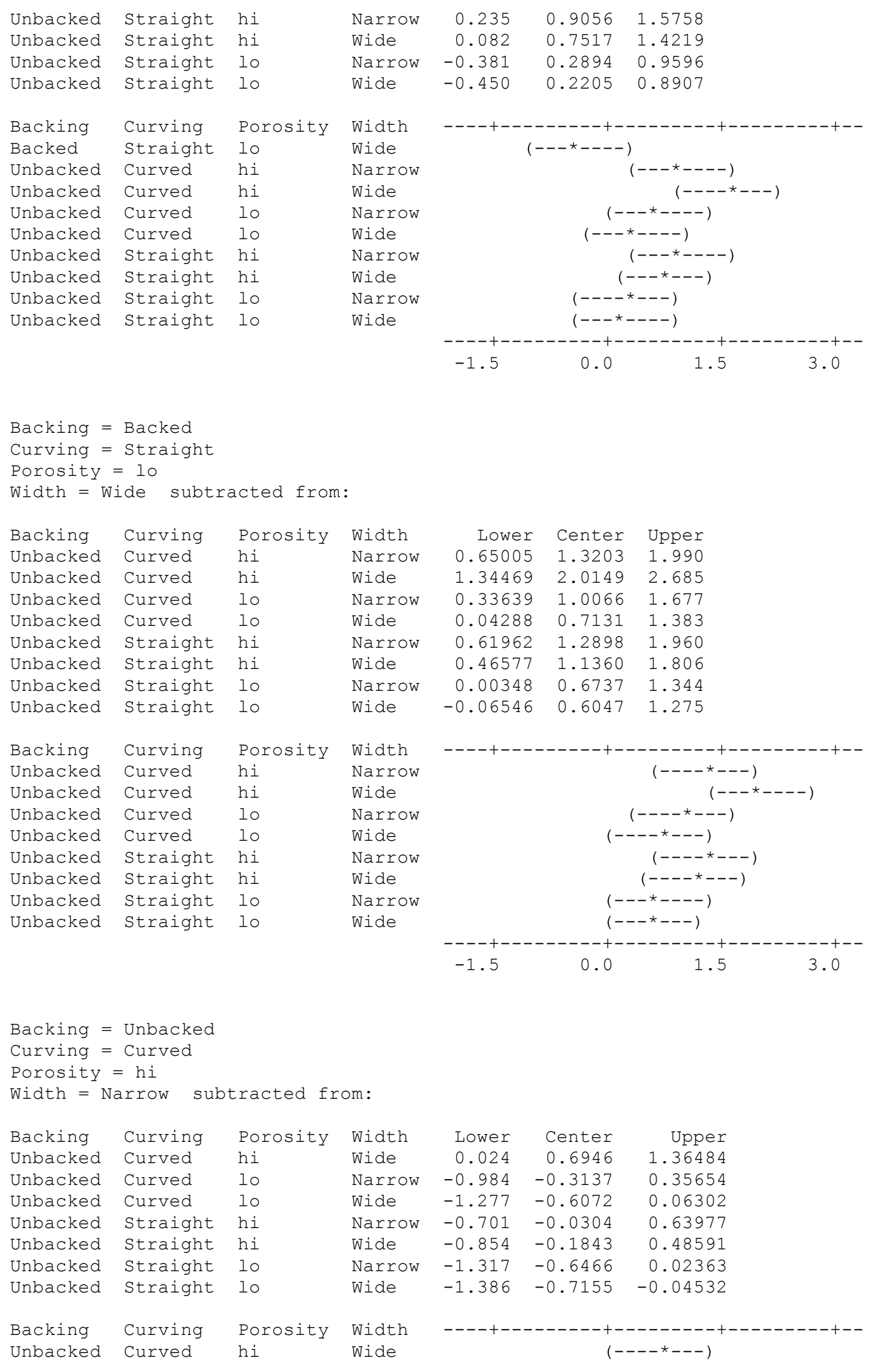




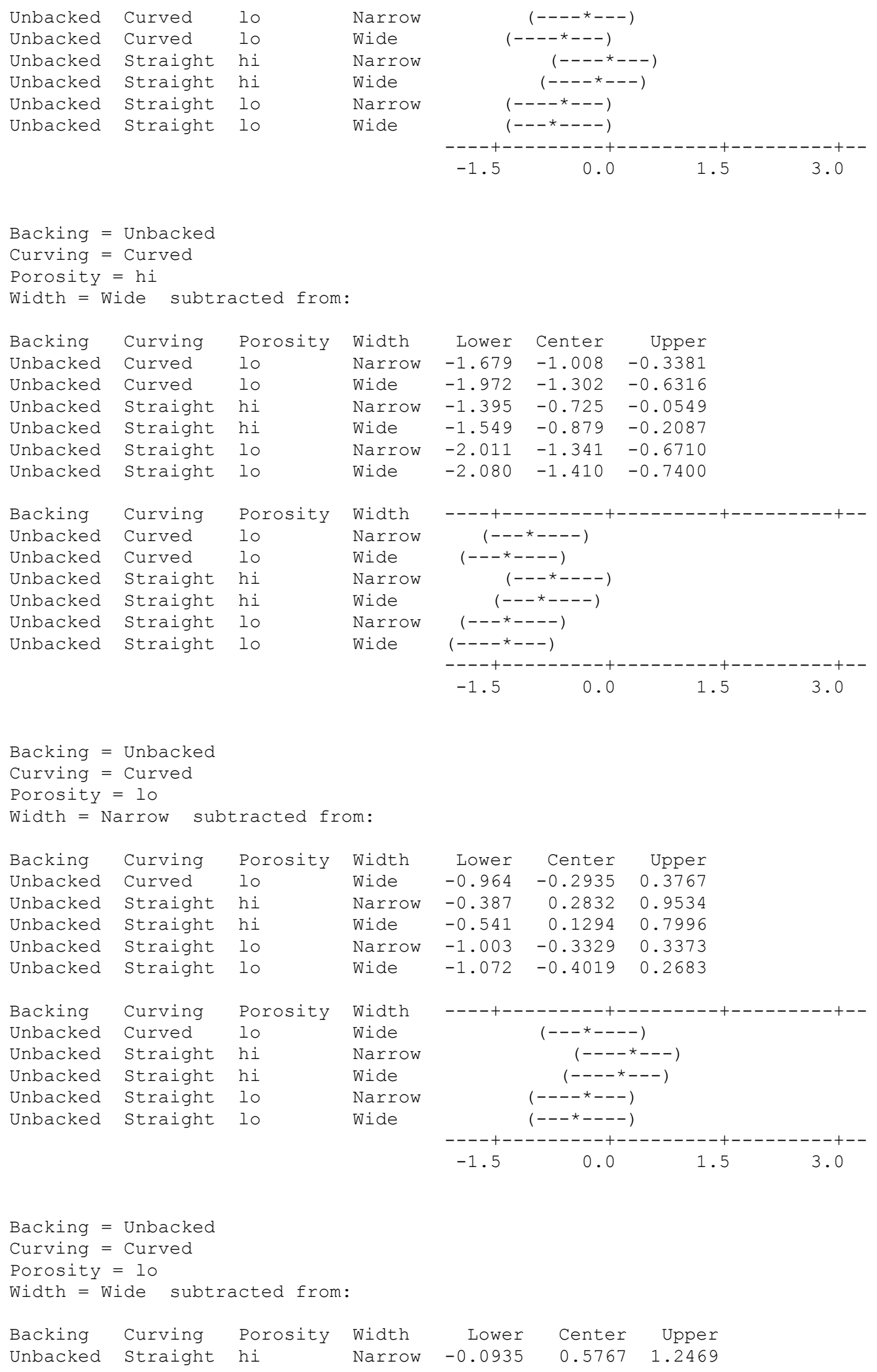




\begin{tabular}{|c|c|c|c|c|c|c|c|}
\hline Unbacked & Straight & hi & Wide & -0.2473 & 0.4229 & 1.0931 & \\
\hline Unbacked & Straight & 10 & Narrow & -0.7096 & -0.0394 & 0.6308 & \\
\hline Unbacked & Straight & 10 & Wide & -0.7785 & -0.1083 & 0.5619 & \\
\hline Backing & Curving & Porosity & Width & 1 & -----+- & --------+- & ---+-- \\
\hline Unbacked & straight & hi & Narrow & & $(--$ & $--\star---)$ & \\
\hline Unbacked & Straight & hi & Wide & & $(---$ & $-\star---)$ & \\
\hline Unbacked & Straight & 10 & Narrow & & $(----\star-$ & $--)$ & \\
\hline Unbacked & Straight & 10 & Wide & & $(---*--$ & $--)$ & \\
\hline & & & & $\begin{array}{c}----+- \\
-1.5\end{array}$ & $\begin{array}{c}---+- \\
0.0\end{array}$ & 1.5 & 3.0 \\
\hline Backing $=$ & Unbacked & & & & & & \\
\hline Curving $=$ & Straight & & & & & & \\
\hline Porosity & $=h i$ & & & & & & \\
\hline Width $=\mathrm{N}$ & arrow sub & Eracted fr & $\mathrm{om}:$ & & & & \\
\hline Backing & Curving & Porosity & Width & Lower & Center & Upper & \\
\hline Unbacked & Straight & $\mathrm{hi}$ & Wide & -0.824 & -0.1539 & 0.51635 & \\
\hline Unbacked & Straight & 10 & Narrow & -1.286 & -0.6161 & 0.05406 & \\
\hline Unbacked & Straight & 10 & Wide & -1.355 & -0.6851 & -0.01488 & \\
\hline Backing & Curving & Porosity & Width & -- & -------+- & --+ & ---+-- \\
\hline Unbacked & straight & hi & Wide & & $(---\star--$ & & \\
\hline Unbacked & Straight & 10 & Narrow & & $---\star---)$ & & \\
\hline Unbacked & Straight & 10 & Wide & $(-$ & $--\star----)$ & & \\
\hline & & & & $\begin{array}{l}-1.5 \\
-1\end{array}$ & 0.0 & $\begin{array}{c}1.5 \\
1.0\end{array}$ & 3.0 \\
\hline Backing $=$ & Unbacked & & & & & & \\
\hline Curving = & Straight & & & & & & \\
\hline Porosity & $=h i$ & & & & & & \\
\hline Width $=\mathrm{W}$ & ide subtr & acted from & & & & & \\
\hline Backing & Curving & Porosity & Width & Lower & Center & Upper & \\
\hline Unbacked & straight & 10 & Narrow & -1.132 & -0.4623 & 0.2079 & \\
\hline Unbacked & Straight & 10 & Wide & -1.201 & -0.5312 & 0.1390 & \\
\hline Backing & Curving & Porosity & Width & ----+-- & --- & - & ---+-- \\
\hline Unbacked & Straight & 10 & Narrow & & $----\star---)$ & & \\
\hline Unbacked & Straight & 10 & Wide & & $---\star----)$ & & \\
\hline & & & & ----+- & -------+- & $---i$ & -- \\
\hline & & & & -1.5 & 0.0 & 1.5 & 3.0 \\
\hline Backing $=$ & Unbacked & & & & & & \\
\hline Curving = & Straight & & & & & & \\
\hline Porosity & $=10$ & & & & & & \\
\hline Width $=\mathrm{N}$ & arrow sub & Eracted fr & $\mathrm{om}:$ & & & & \\
\hline Backing & Curving & Porosity & Width & Lower & Center & Upper & \\
\hline Unbacked & Straight & 10 & Wide & -0.7391 & -0.06894 & 0.6013 & \\
\hline Backing & Curving & Porosity & Width & & $=---2--+--1$ & ------+ & --+-- \\
\hline Unbacked & Straight & 10 & Wide & & $(----\star--$ & & \\
\hline & & & & $\begin{array}{l}----+-- \\
-1.5\end{array}$ & $\begin{array}{c}----+-- \\
0.0\end{array}$ & $\begin{array}{c}1.5 \\
-1 .-+-\end{array}$ & 3.0 \\
\hline
\end{tabular}

Tukey Simultaneous Tests

Response Variable log(time) 


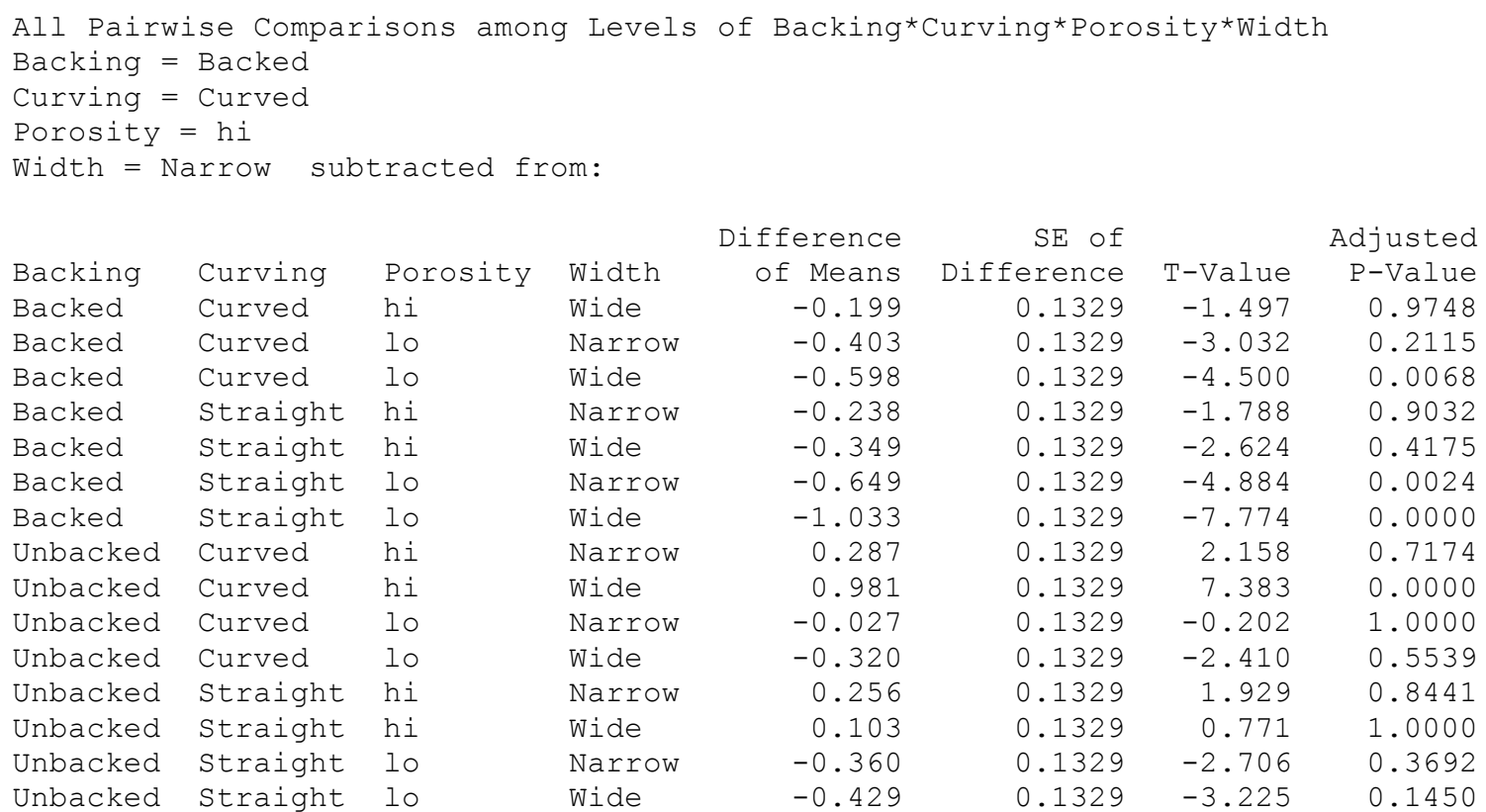

Backing $=$ Backed

Curving $=$ Curved

Porosity $=$ hi

Width = Wide subtracted from:

$\begin{array}{llllr}\text { Backing } & \text { Curving } & \text { Porosity } & \text { Width } & \text { of Means } \\ \text { Backed } & \text { Curved } & \text { lo } & \text { Narrow } & -0.2040 \\ \text { Backed } & \text { Curved } & \text { lo } & \text { Wide } & -0.3992 \\ \text { Backed } & \text { Straight } & \text { hi } & \text { Narrow } & -0.0387 \\ \text { Backed } & \text { Straight } & \text { hi } & \text { Wide } & -0.1498 \\ \text { Backed } & \text { Straight } & \text { lo } & \text { Narrow } & -0.4502 \\ \text { Backed } & \text { Straight } & \text { lo } & \text { Wide } & -0.8344 \\ \text { Unbacked } & \text { Curved } & \text { hi } & \text { Narrow } & 0.4858 \\ \text { Unbacked } & \text { Curved } & \text { hi } & \text { Wide } & 1.1805 \\ \text { Unbacked } & \text { Curved } & \text { lo } & \text { Narrow } & 0.1722 \\ \text { Unbacked } & \text { Curved } & \text { lo } & \text { Wide } & -0.1213 \\ \text { Unbacked } & \text { Straight } & \text { hi } & \text { Narrow } & 0.4554 \\ \text { Unbacked } & \text { Straight } & \text { hi } & \text { Wide } & 0.3016 \\ \text { Unbacked } & \text { Straight } & \text { lo } & \text { Narrow } & -0.1607 \\ \text { Unbacked } & \text { Straight } & \text { lo } & \text { Wide } & -0.2297\end{array}$

$\begin{array}{rrr}\text { SE of } & & \begin{array}{r}\text { Adjusted } \\ \text { P-Value }\end{array} \\ 0.1329 & -1.535 & 0.9691 \\ 0.1329 & -3.003 & 0.2231 \\ 0.1329 & -0.291 & 1.0000 \\ 0.1329 & -1.127 & 0.9984 \\ 0.1329 & -3.386 & 0.1034 \\ 0.1329 & -6.277 & 0.0001 \\ 0.1329 & 3.655 & 0.0567 \\ 0.1329 & 8.880 & 0.0000 \\ 0.1329 & 1.295 & 0.9933 \\ 0.1329 & -0.913 & 0.9999 \\ 0.1329 & 3.426 & 0.0949 \\ 0.1329 & 2.268 & 0.6469 \\ 0.1329 & -1.209 & 0.9966 \\ 0.1329 & -1.728 & 0.9235\end{array}$

Backing $=$ Backed

Curving $=$ Curved

Porosity $=10$

Width $=$ Narrow subtracted from:

$\begin{array}{llllr}\text { Backing } & \text { Curving } & \text { Porosity } & \text { Width } & \begin{array}{r}\text { Difference } \\ \text { of Means }\end{array} \\ \text { Backed } & \text { Curved } & \text { lo } & \text { Wide } & -0.1952 \\ \text { Backed } & \text { Straight } & \text { hi } & \text { Narrow } & 0.1653 \\ \text { Backed } & \text { Straight } & \text { hi } & \text { Wide } & 0.0542 \\ \text { Backed } & \text { Straight } & \text { lo } & \text { Narrow } & -0.2462 \\ \text { Backed } & \text { Straight } & \text { lo } & \text { Wide } & -0.6304 \\ \text { Unbacked } & \text { Curved } & \text { hi } & \text { Narrow } & 0.6898 \\ \text { Unbacked } & \text { Curved } & \text { hi } & \text { Wide } & 1.3845 \\ \text { Unbacked } & \text { Curved } & \text { lo } & \text { Narrow } & 0.3762\end{array}$

\begin{tabular}{|c|c|c|}
\hline SE of & & Adjusted \\
\hline Difference & T-Value & P-Value \\
\hline 0.1329 & -1.469 & 0.9787 \\
\hline 0.1329 & 1.244 & 0.995 \\
\hline 0.1329 & 0.408 & 1.0 \\
\hline 0.1329 & -1.852 & 0.87 \\
\hline 0.1329 & -4.742 & 0.00 \\
\hline 0.1329 & 5.189 & 0.00 \\
\hline 0.1329 & 10.415 & 0.0 \\
\hline & 0 & \\
\hline
\end{tabular}




\begin{tabular}{|c|c|c|c|c|c|c|c|}
\hline Unbacked & Curved & 10 & Wide & 0.0827 & 0.1329 & 0.622 & 1.0000 \\
\hline Unbacked & Straight & hi & Narrow & 0.6594 & 0.1329 & 4.960 & 0.0020 \\
\hline Unbacked & Straight & hi & Wide & 0.5055 & 0.1329 & 3.803 & 0.0399 \\
\hline Unbacked & Straight & 10 & Narrow & 0.0433 & 0.1329 & 0.325 & 1.0000 \\
\hline Unbacked & Straight & 10 & Wide & -0.0257 & 0.1329 & -0.193 & 1.0000 \\
\hline
\end{tabular}

Backing $=$ Backed

Curving $=$ Curved

Porosity = lo

Width = Wide subtracted from:

\begin{tabular}{|c|c|c|c|c|c|c|c|}
\hline Backing & Curving & Porosity & Width & $\begin{array}{r}\text { Difference } \\
\text { of Means }\end{array}$ & $\begin{array}{r}\text { SE of } \\
\text { Difference }\end{array}$ & T-Value & $\begin{array}{l}\text { Adjusted } \\
\text { P-Value }\end{array}$ \\
\hline Backed & Straight & hi & Narrow & 0.3605 & 0.1329 & 2.712 & 0.3659 \\
\hline Backed & Straight & hi & Wide & 0.2494 & 0.1329 & 1.876 & 0.8681 \\
\hline Backed & Straight & 10 & Narrow & -0.0510 & 0.1329 & -0.383 & 1.0000 \\
\hline Backed & straight & 10 & Wide & -0.4352 & 0.1329 & -3.274 & 0.1312 \\
\hline Unbacked & Curved & hi & Narrow & 0.8851 & 0.1329 & 6.658 & 0.0001 \\
\hline Unbacked & Curved & hi & Wide & 1.5797 & 0.1329 & 11.883 & 0.0000 \\
\hline Unbacked & Curved & 10 & Narrow & 0.5714 & 0.1329 & 4.298 & 0.0116 \\
\hline Unbacked & Curved & 10 & Wide & 0.2779 & 0.1329 & 2.090 & 0.7578 \\
\hline Unbacked & Straight & hi & Narrow & 0.8546 & 0.1329 & 6.429 & 0.0001 \\
\hline Unbacked & Straight & hi & Wide & 0.7008 & 0.1329 & 5.272 & 0.0009 \\
\hline nbacked & Straight & 10 & Narrow & 0.2385 & 0.1329 & 1.794 & 0.90 \\
\hline nbacked & Straight & 10 & Wide & 0.1695 & 0.1329 & 1.275 & 0.99 \\
\hline
\end{tabular}

Backing $=$ Backed

Curving = Straight

Porosity $=$ hi

Width = Narrow subtracted from:

\begin{tabular}{|c|c|c|c|c|c|c|c|}
\hline Backing & Curving & Porosity & Width & $\begin{array}{r}\text { Difference } \\
\text { of Means }\end{array}$ & $\begin{array}{r}\text { SE of } \\
\text { Difference }\end{array}$ & T-Value & $\begin{array}{r}\text { Adjusted } \\
\text { P-Value }\end{array}$ \\
\hline Backed & Straight & hi & Wide & -0.1111 & 0.1329 & -0.836 & 0.9999 \\
\hline Backed & Straight & 10 & Narrow & -0.4115 & 0.1329 & -3.095 & 0.1874 \\
\hline Backed & Straight & 10 & Wide & -0.7957 & 0.1329 & -5.986 & 0.0002 \\
\hline Unbacked & Curved & hi & Narrow & 0.5245 & 0.1329 & 3.946 & 0.0282 \\
\hline Unbacked & Curved & hi & Wide & 1.2192 & 0.1329 & 9.171 & 0.0000 \\
\hline Unbacked & Curved & 10 & Narrow & 0.2109 & 0.1329 & 1.586 & 0.9596 \\
\hline Unbacked & Curved & 10 & Wide & -0.0827 & 0.1329 & -0.622 & 1.0000 \\
\hline Unbacked & Straight & hi & Narrow & 0.4941 & 0.1329 & 3.717 & 0.0490 \\
\hline Unbacked & Straight & hi & Wide & 0.3402 & 0.1329 & 2.559 & 0.4574 \\
\hline Unbacked & Straight & 10 & Narrow & -0.1221 & 0.1329 & -0.918 & 0.9998 \\
\hline Unbacked & Straight & 10 & Wide & -0.1910 & 0.1329 & -1.437 & 0.9824 \\
\hline
\end{tabular}

Backing $=$ Backed

Curving $=$ Straight

Porosity $=$ hi

Width = Wide subtracted from:

\begin{tabular}{|c|c|c|c|c|c|c|c|}
\hline Backing & Curving & Porosity & Width & $\begin{array}{l}\text { Difference } \\
\text { of Means }\end{array}$ & $\begin{array}{r}\text { SE of } \\
\text { Difference }\end{array}$ & T-Value & $\begin{array}{l}\text { Adjusted } \\
\text { P-Value }\end{array}$ \\
\hline Backed & Straight & 10 & Narrow & -0.3004 & 0.1329 & -2.259 & 0.6528 \\
\hline Backed & Straight & 10 & Wide & -0.6846 & 0.1329 & -5.150 & 0.0012 \\
\hline Unbacked & Curved & hi & Narrow & 0.6357 & 0.1329 & 4.782 & 0.0032 \\
\hline Unbacked & Curved & hi & Wide & 1.3303 & 0.1329 & 10.007 & 0.0000 \\
\hline Unbacked & Curved & 10 & Narrow & 0.3220 & 0.1329 & 2.422 & 0.5459 \\
\hline Unbacked & Curved & 10 & Wide & 0.0285 & 0.1329 & 0.214 & 1.0000 \\
\hline Unbacked & Straight & hi & Narrow & 0.6052 & 0.1329 & 4.553 & 0.0059 \\
\hline Unbacked & Straight & hi & Wide & 0.4514 & 0.1329 & 3.395 & 0.1014 \\
\hline
\end{tabular}




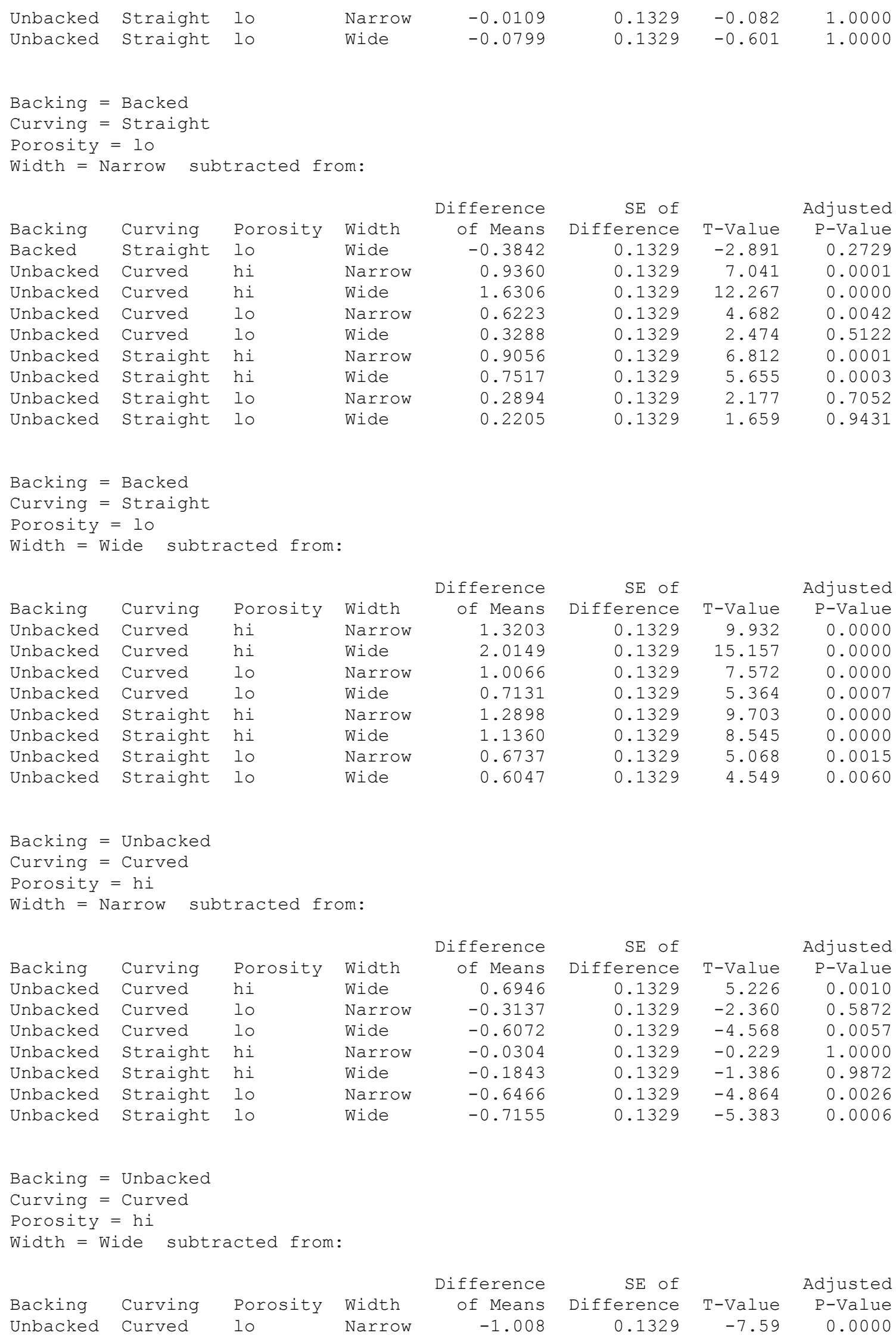




$\begin{array}{llllllll}\text { Unbacked } & \text { Curved } & \text { lo } & \text { Wide } & -1.302 & 0.1329 & -9.79 & 0.0000 \\ \text { Unbacked } & \text { Straight } & \text { hi } & \text { Narrow } & -0.725 & 0.1329 & -5.45 & 0.0005 \\ \text { Unbacked } & \text { Straight } & \text { hi } & \text { Wide } & -0.879 & 0.1329 & -6.61 & 0.0001 \\ \text { Unbacked } & \text { Straight } & \text { lo } & \text { Narrow } & -1.341 & 0.1329 & -10.09 & 0.0000 \\ \text { Unbacked } & \text { Straight } & \text { lo } & \text { Wide } & -1.410 & 0.1329 & -10.61 & 0.0000\end{array}$

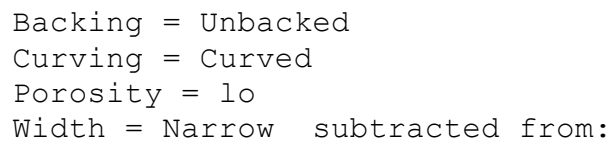

Difference of Means 0.5767 0.4229 $-0.0394$ $-0.1083$

Difference of Means $-0.2935$

0.2832

0.1294

$-0.3329$

$-0.4019$
SE of

Difference

0.1329

0.1329

0.1329

0.1329

0.1329
T-Value

$-2.208$

2.131

0.973

$-2.504$

$-3.023$
Adjusted

P-Value

0.6858

0.7339

0.9997

0.4924

0.2150

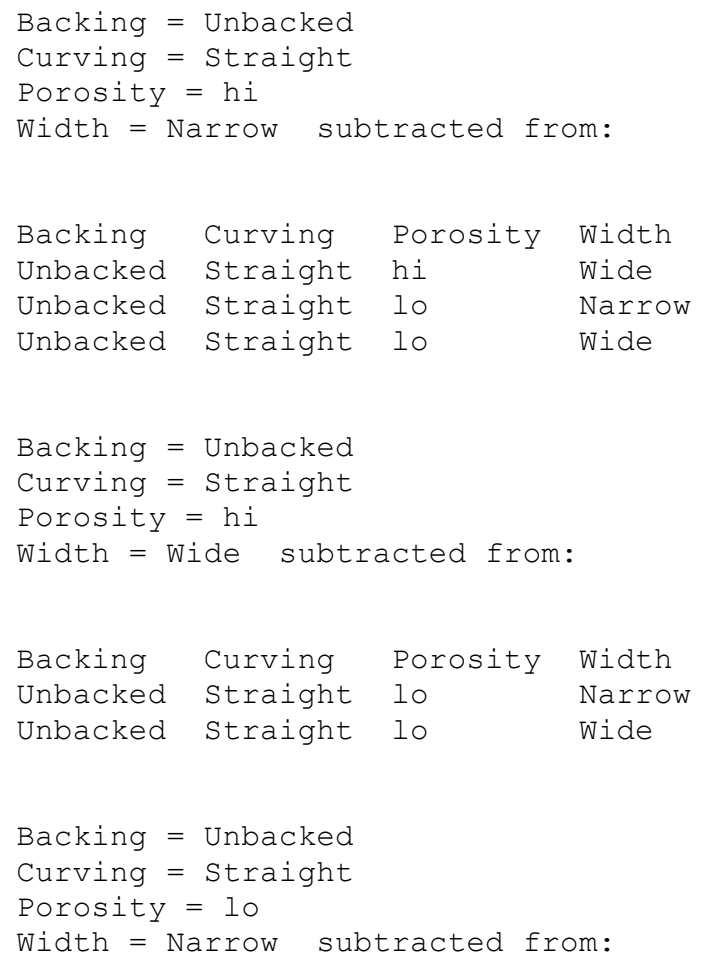

Difference of Means $-0.1539$ $-0.6161$ $-0.6851$

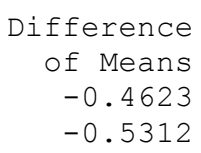

Adjusted P-Value 0.0847 0.0249 


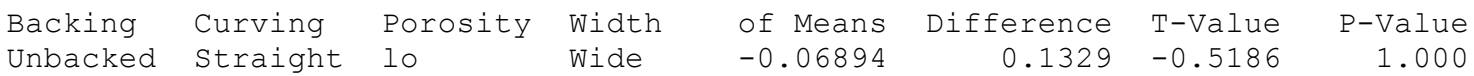

The following values are for the Cook's distances for the initial statistical model.

\begin{tabular}{|c|c|}
\hline \multicolumn{2}{|c|}{ COOK1 } \\
\hline 0.000000 & 0.033003 \\
\hline 0.005474 & 0.000517 \\
\hline 0.000763 & 0.026726 \\
\hline 0.029125 & 0.005063 \\
\hline 0.002838 & 0.000085 \\
\hline 0.023835 & 0.000331 \\
\hline 0.000024 & 0.054164 \\
\hline 0.006867 & 0.000246 \\
\hline 0.207713 & 0.231658 \\
\hline 0.004975 & 0.000601 \\
\hline 0.000107 & 0.014258 \\
\hline 0.050588 & 0.040064 \\
\hline 0.134668 & 0.000006 \\
\hline 0.000097 & 0.076924 \\
\hline 0.003388 & 0.008772 \\
\hline 0.006073 & 0.001996 \\
\hline 0.000709 & 0.052204 \\
\hline 0.002384 & 0.173713 \\
\hline 0.000041 & 0.000454 \\
\hline 0.010934 & 0.000002 \\
\hline 0.088755 & 0.020970 \\
\hline 0.000683 & 0.001944 \\
\hline 0.013208 & 0.09220 \\
\hline 0.003924 & \\
\hline 0.179517 & \\
\hline 0.000001 & \\
\hline 0.001676 & \\
\hline 0.000249 & \\
\hline 0.021316 & \\
\hline 0.199699 & \\
\hline 0.000351 & \\
\hline 0.000016 & \\
\hline 0.207713 & \\
\hline 0.050588 & \\
\hline 0.004975 & \\
\hline 0.199699 & \\
\hline 0.004520 & \\
\hline 0.070392 & \\
\hline 0.000009 & \\
\hline 0.057922 & \\
\hline 0.001515 & \\
\hline
\end{tabular}




\section{ANOVA Output for the Tukey Model for Significant Interactions}

\section{COMPARISON DATA}

Grouping Information Using Tukey Method and 99.7\% Confidence

\begin{tabular}{|c|c|c|c|c|c|c|c|c|c|c|}
\hline Backing & Curving & Porosity & Width & $\mathrm{N}$ & Mean & \multicolumn{5}{|c|}{ Grouping } \\
\hline Unbacked & Curved & hi & Wide & 4 & 2.8 & A & & & & \\
\hline Unbacked & Curved & hi & Narrow & 4 & 2.1 & B & & & & \\
\hline Unbacked & Straight & hi & Narrow & 4 & 2.1 & B & $\mathrm{C}$ & & & \\
\hline Unbacked & Straight & hi & Wide & 4 & 1.9 & B & $\mathrm{C}$ & D & & \\
\hline Backed & Curved & hi & Narrow & 4 & 1.8 & B & C & D & $E$ & \\
\hline Unbacked & Curved & 10 & Narrow & 4 & 1.8 & B & C & D & $E$ & $\mathrm{~F}$ \\
\hline Backed & Curved & hi & Wide & 4 & 1.6 & B & $\mathrm{C}$ & D & $E$ & $\mathrm{~F}$ \\
\hline Backed & Straight & hi & Narrow & 4 & 1.6 & B & $\mathrm{C}$ & $\mathrm{D}$ & $E$ & $\mathrm{~F}$ \\
\hline Unbacked & Curved & 10 & Wide & 4 & 1.5 & B & $\mathrm{C}$ & D & $\mathrm{E}$ & $\mathrm{F}$ \\
\hline Backed & Straight & hi & Wide & 4 & 1.5 & & $\mathrm{C}$ & D & $\mathrm{E}$ & $\mathrm{F}$ \\
\hline Unbacked & Straight & 10 & Narrow & 4 & 1.5 & & C & D & $\mathrm{E}$ & $\mathrm{F}$ \\
\hline Backed & Curved & 10 & Narrow & 4 & 1.4 & & & D & $\mathrm{E}$ & $\mathrm{F} C$ \\
\hline Unbacked & Straight & 10 & Wide & 4 & 1.4 & & & D & $E$ & $\mathrm{~F}$ \\
\hline Backed & Curved & 10 & Wide & 4 & 1.2 & & & & $E$ & $\mathrm{~F}$ \\
\hline Backed & Straight & 10 & Narrow & 4 & 1.2 & & & & & $\mathrm{~F}$ \\
\hline Backed & Straight & 10 & Wide & 4 & 0.8 & & & & & \\
\hline
\end{tabular}

Means that do not share a letter are significantly different.

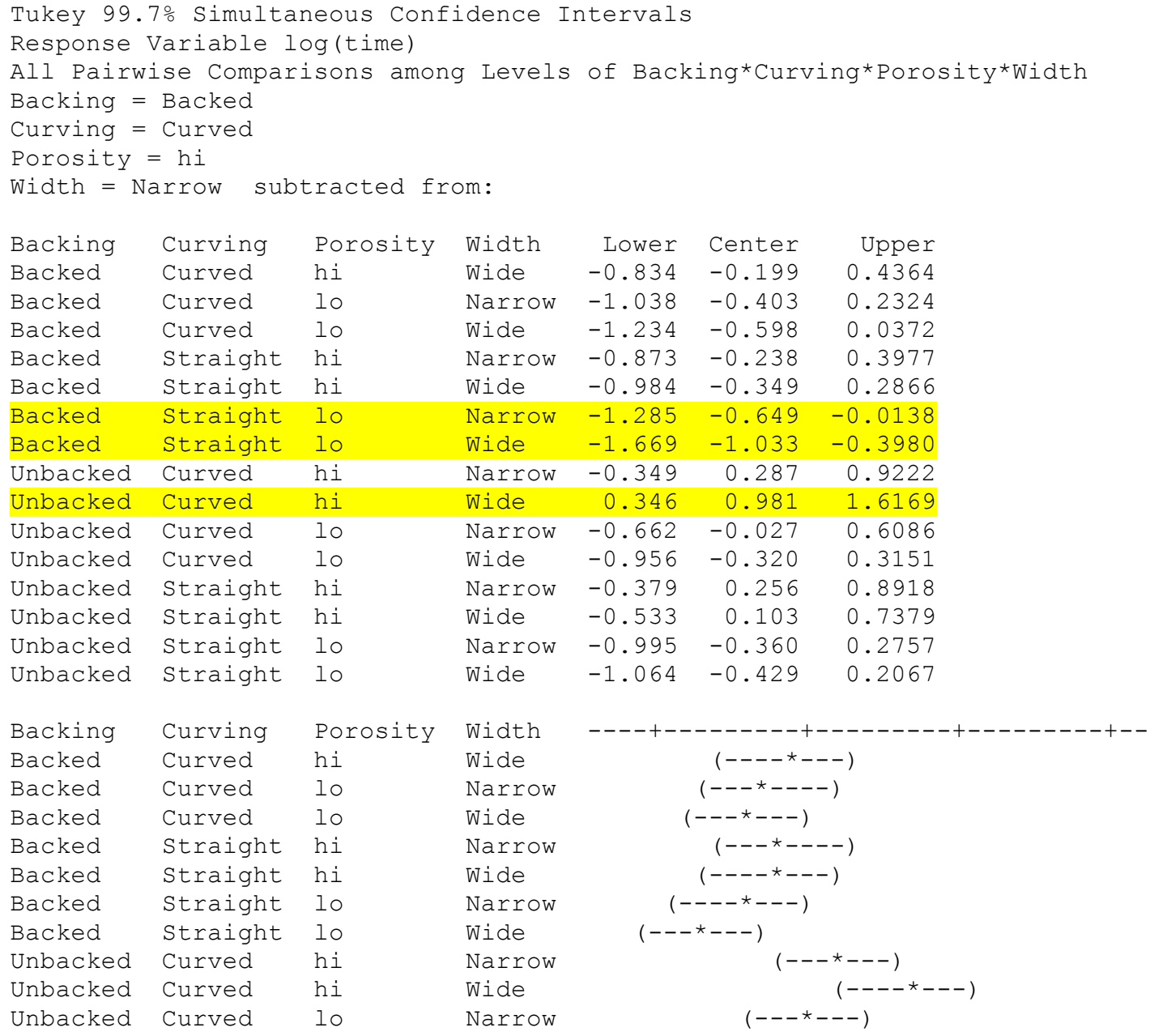




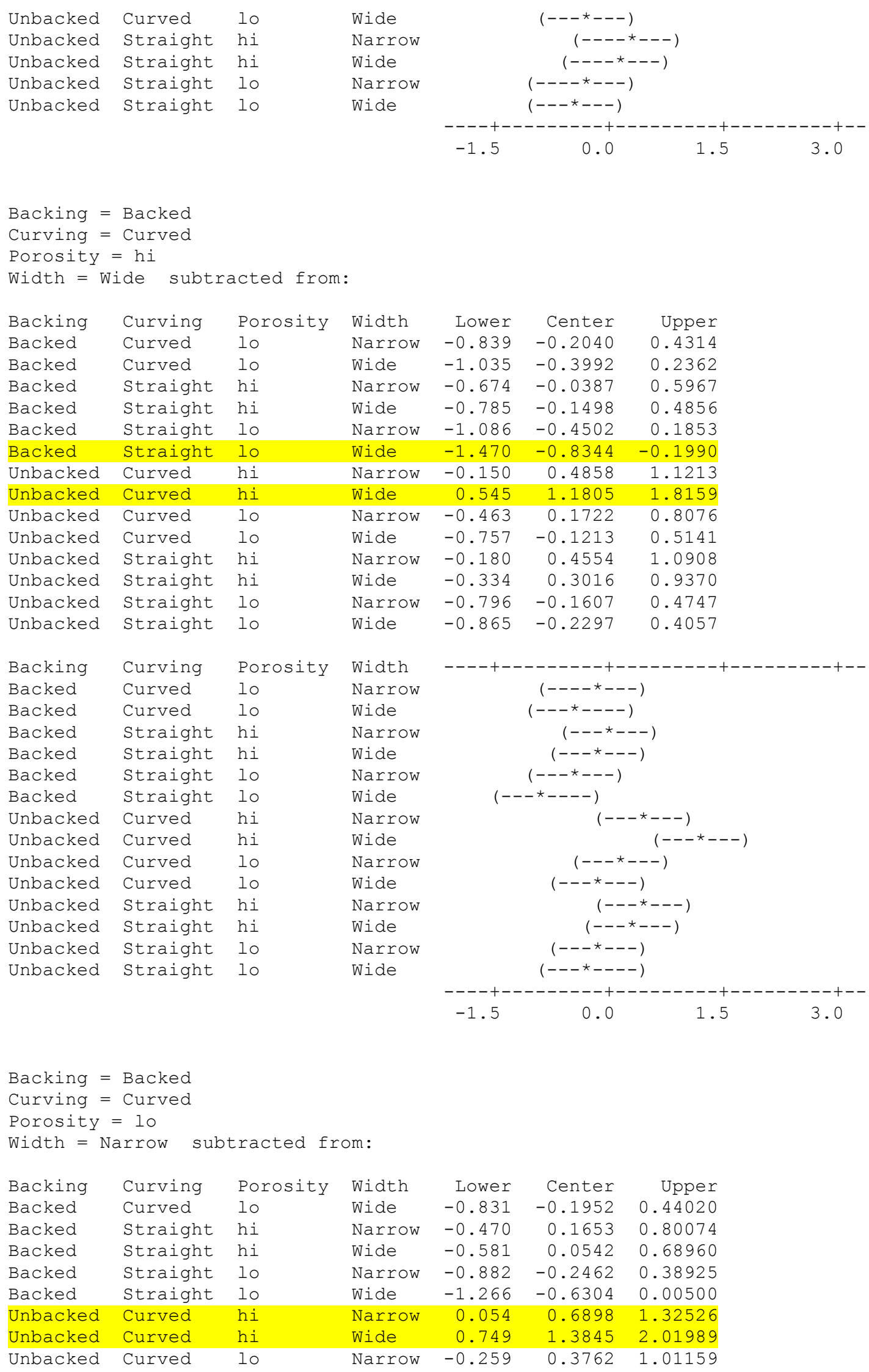




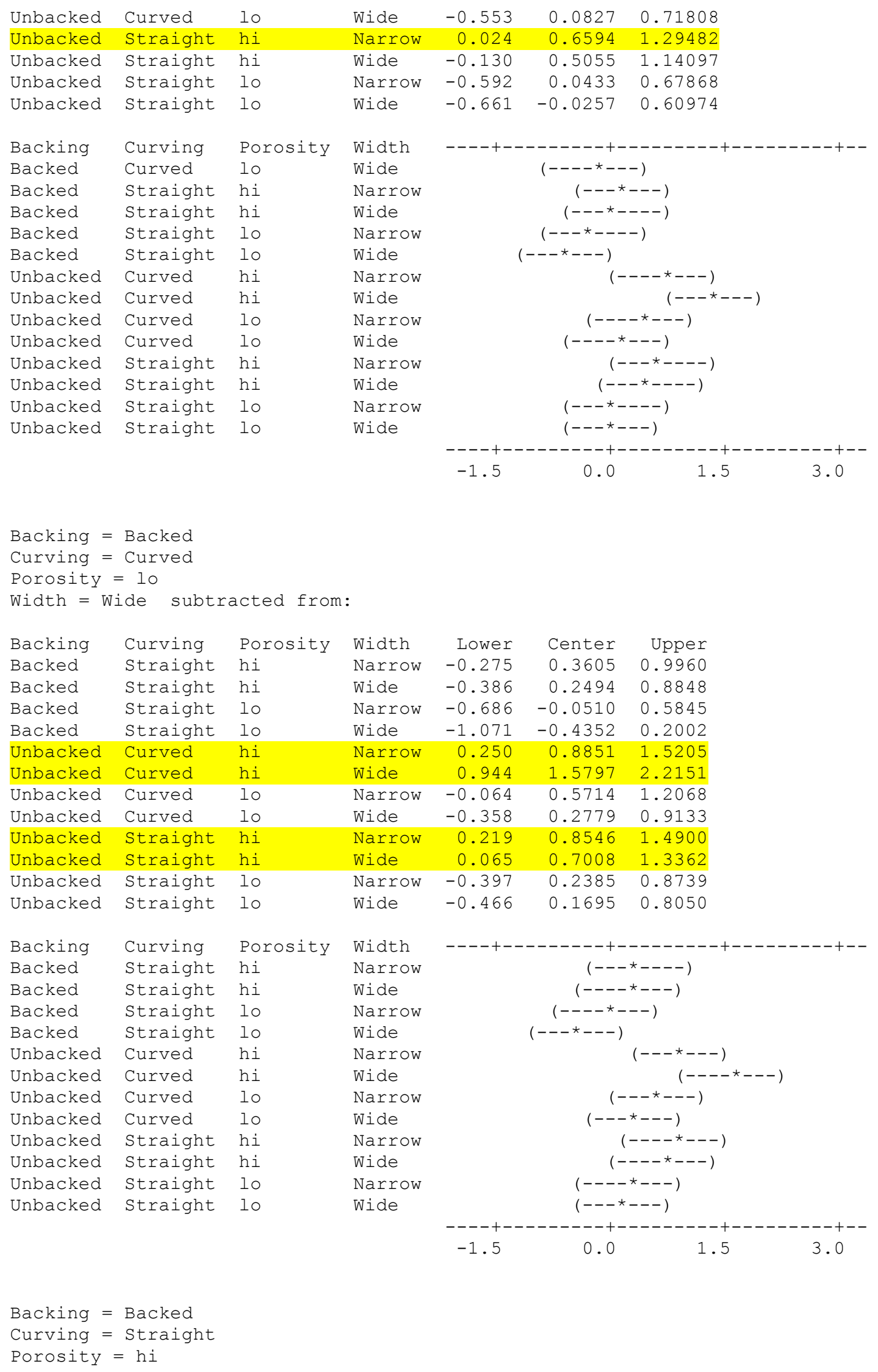




\begin{tabular}{|c|c|c|c|c|c|c|}
\hline Backing & Curving & Porosity & Width & Lower & Center & Upper \\
\hline Backed & straight & hi & Wide & -0.747 & -0.1111 & 0.5243 \\
\hline Backed & Straight & 10 & Narrow & -1.047 & -0.4115 & 0.2239 \\
\hline Backed & Straight & 10 & Wide & -1.431 & -0.7957 & -0.1603 \\
\hline Unbacked & Curved & hi & Narrow & -0.111 & 0.5245 & 1.1599 \\
\hline Unbacked & Curved & hi & Wide & 0.584 & 1.2192 & 1.8546 \\
\hline Unbacked & Curved & 10 & Narrow & -0.425 & 0.2109 & 0.8463 \\
\hline Unbacked & Curved & 10 & Wide & -0.718 & -0.0827 & 0.5528 \\
\hline Unbacked & Straight & hi & Narrow & -0.141 & 0.4941 & 1.1295 \\
\hline Unbacked & Straight & hi & Wide & -0.295 & 0.3402 & 0.9756 \\
\hline Unbacked & Straight & 10 & Narrow & -0.757 & -0.1221 & 0.5134 \\
\hline Unbacked & Straight & 10 & Wide & -0.826 & -0.1910 & 0.4444 \\
\hline Backing & Curving & Porosity & Width & & & \\
\hline Backed & straight & hi & Wide & \multicolumn{3}{|c|}{$(---\star---)$} \\
\hline Backed & Straight & 10 & Narrow & \multicolumn{3}{|c|}{$(---\star---)$} \\
\hline Backed & Straight & 10 & Wide & \multicolumn{3}{|c|}{$(----\star---)$} \\
\hline Unbacked & Curved & hi & Narrow & \multicolumn{3}{|c|}{$(---\star----)$} \\
\hline Unbacked & Curved & hi & Wide & \multicolumn{3}{|c|}{$(---\star---)$} \\
\hline Unbacked & Curved & 10 & Narrow & \multicolumn{3}{|c|}{$(---\star----)$} \\
\hline Unbacked & Curved & 10 & Wide & \multicolumn{3}{|c|}{$(---\star----)$} \\
\hline Unbacked & Straight & hi & Narrow & \multicolumn{3}{|c|}{$(---\star----)$} \\
\hline Unbacked & Straight & hi & Wide & \multicolumn{3}{|c|}{$(---\star----)$} \\
\hline Unbacked & Straight & 10 & Narrow & \multicolumn{3}{|c|}{$(---\star---)$} \\
\hline Unbacked & Straight & 10 & Wide & \multicolumn{3}{|c|}{$(----*---)$} \\
\hline & & & & \multicolumn{3}{|c|}{$\begin{array}{ccc}-1.5 & 0.0 & 1.5\end{array}$} \\
\hline
\end{tabular}

Backing $=$ Backed

Curving = Straight

Porosity $=\mathrm{hi}$

Width = Wide subtracted from:

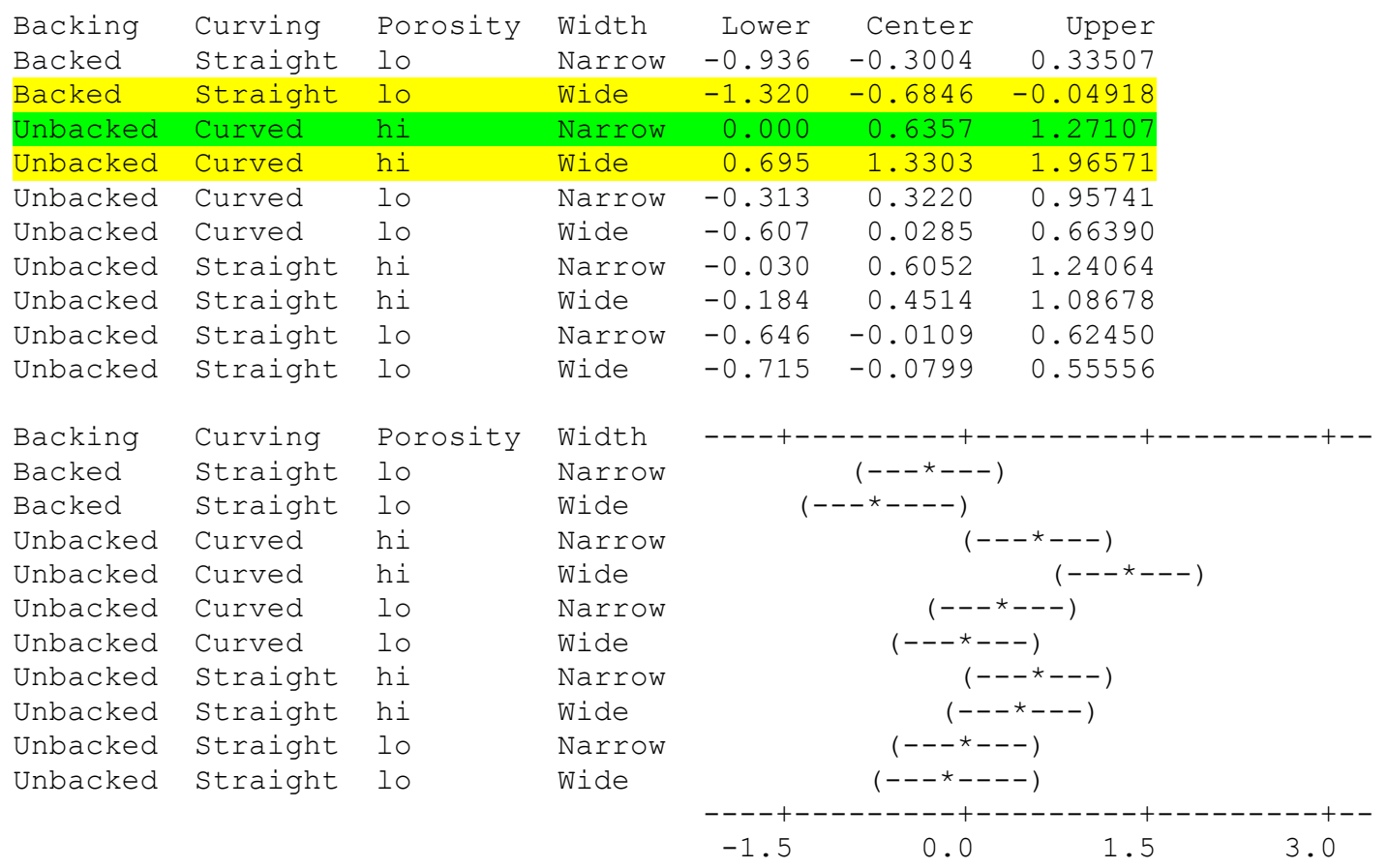




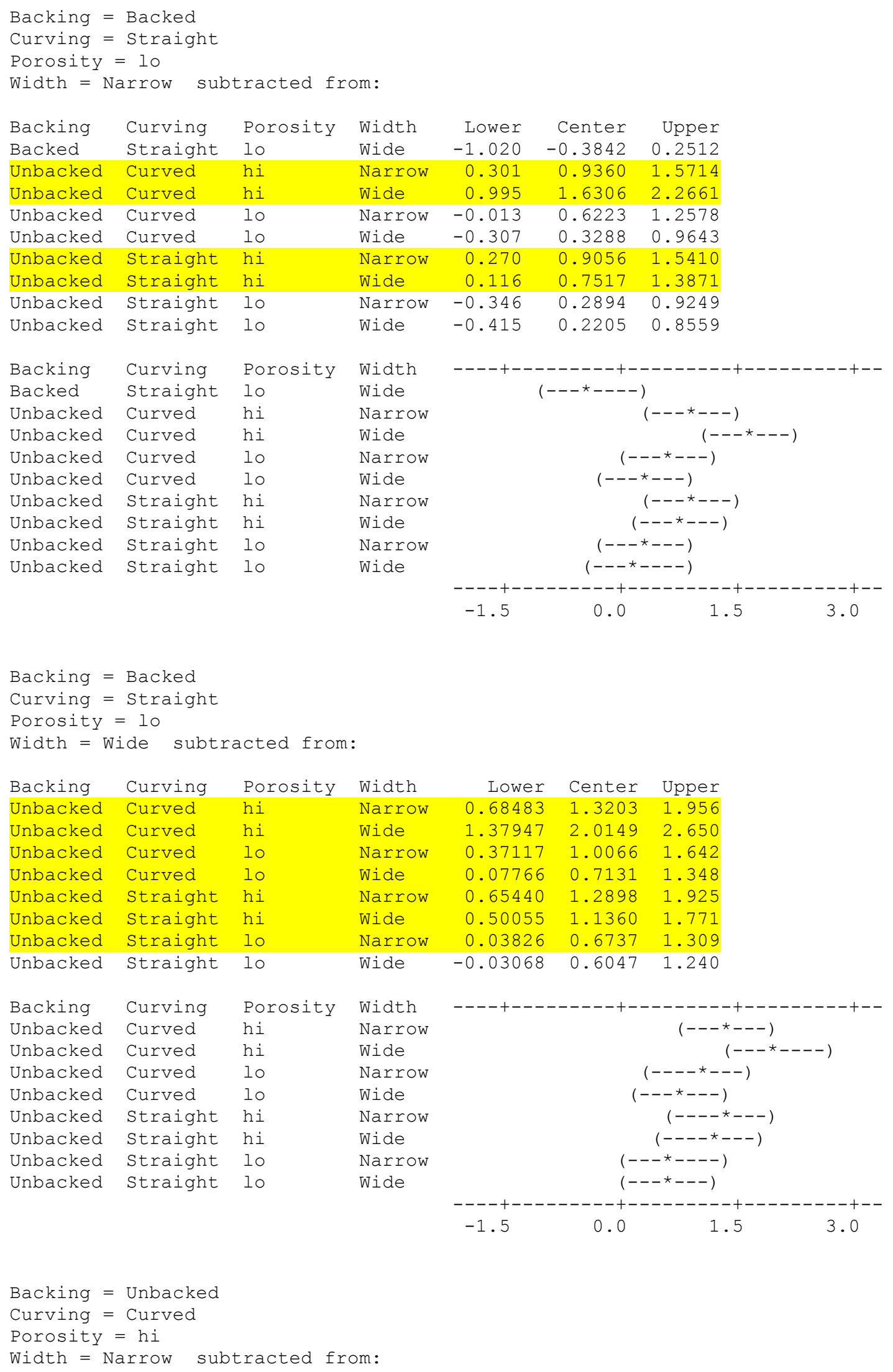




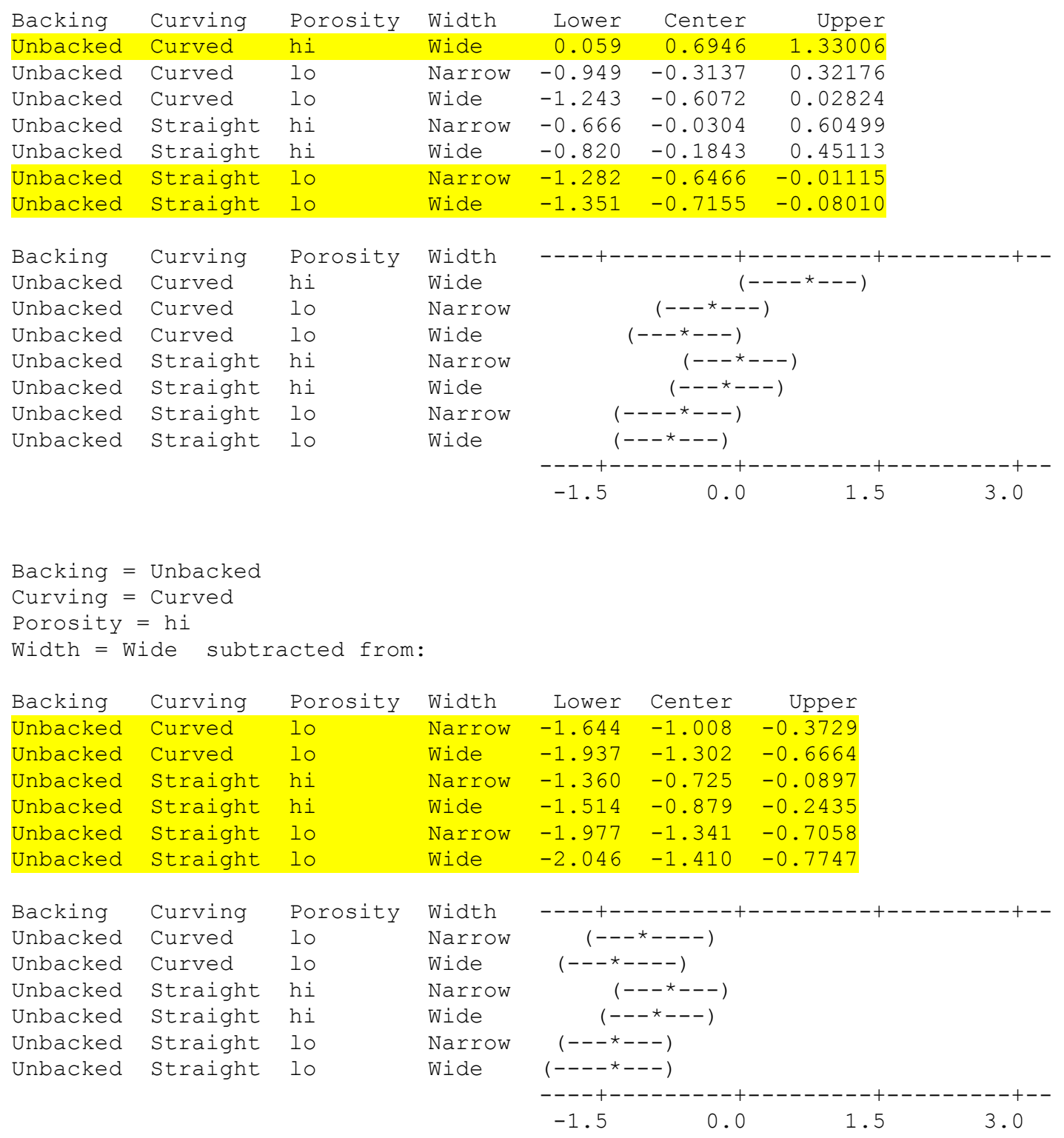

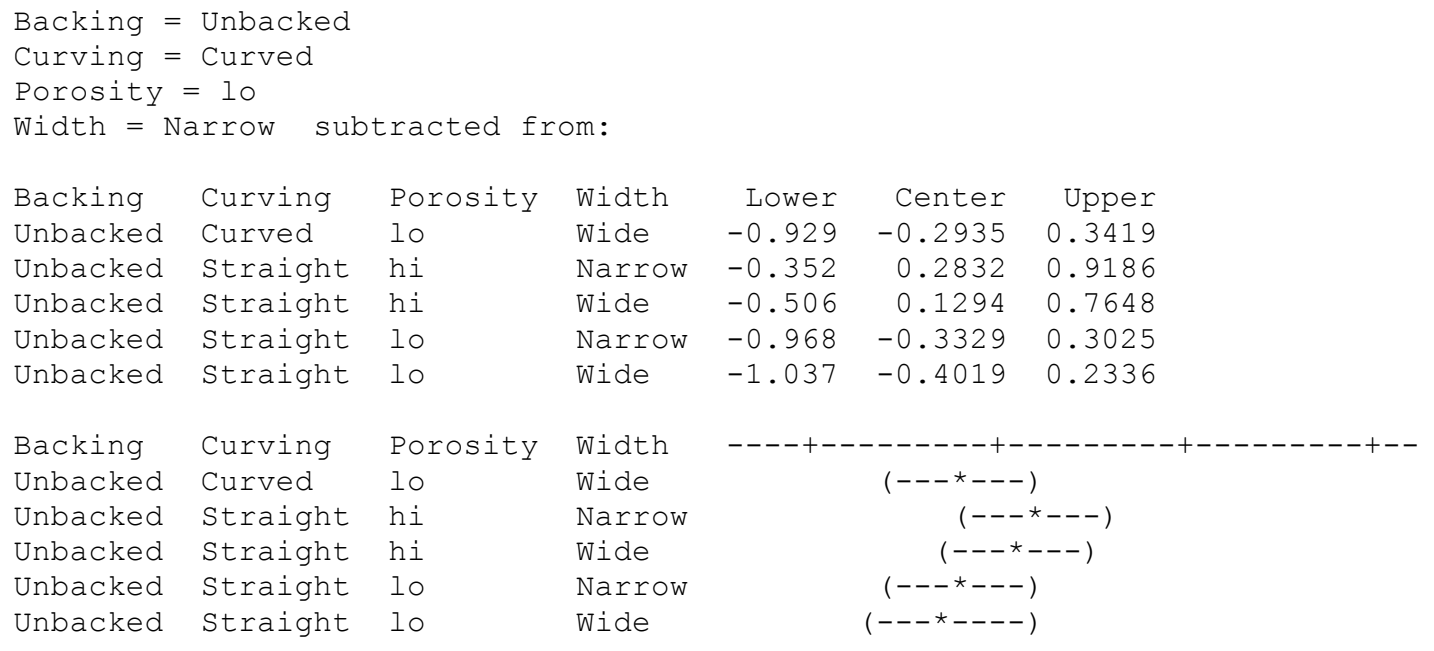




\begin{tabular}{|c|c|}
\hline-1.5 & 0.0 \\
\hline
\end{tabular}

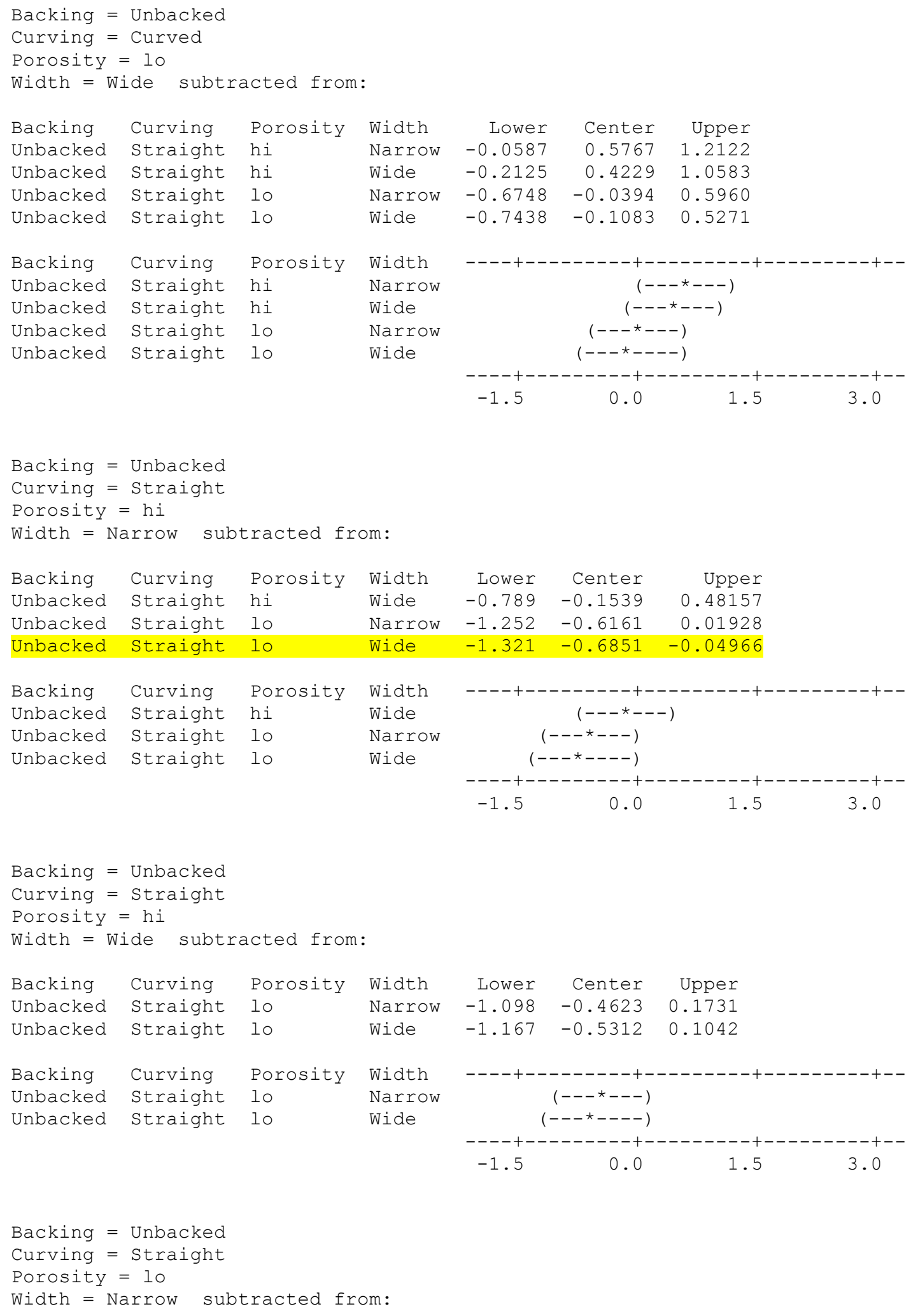

Backing $=$ Unbacked

Curving = Straight

Porosity $=$ hi

Width = Wide subtracted from:

\begin{tabular}{|c|c|c|c|c|c|c|}
\hline Backing & Curving & Porosity & Width & Lower & Center & Upper \\
\hline Unbacked & Straight & 10 & Narrow & -1.098 & -0.4623 & 0.1731 \\
\hline Unbacked & straight & 10 & Wide & -1.167 & -0.5312 & 0.1042 \\
\hline Backing & Curving & Porosity & Width & & & \\
\hline Unbacked & Straight & 10 & Narrow & & $(---\star---)$ & \\
\hline Unbacked & Straight & 10 & Wide & & $---\star----)$ & \\
\hline & & & & -1.5 & 0.0 & 1.5 \\
\hline
\end{tabular}

Backing $=$ Unbacked

Curving = Straight

Porosity $=10$

Width = Narrow subtracted from: 


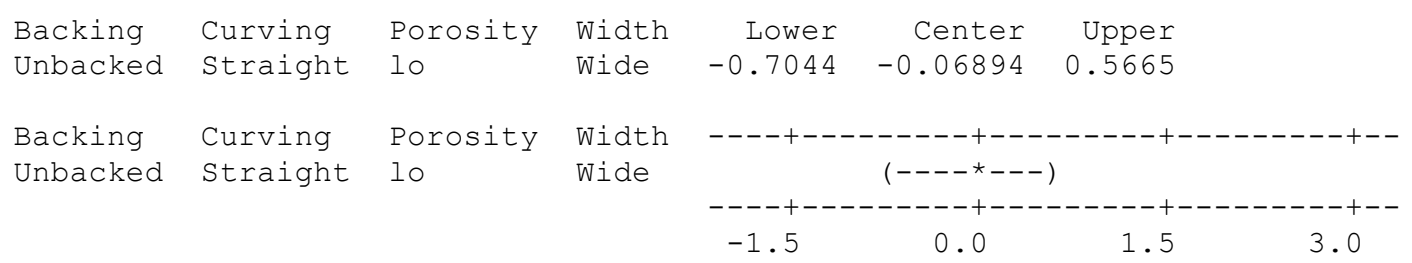




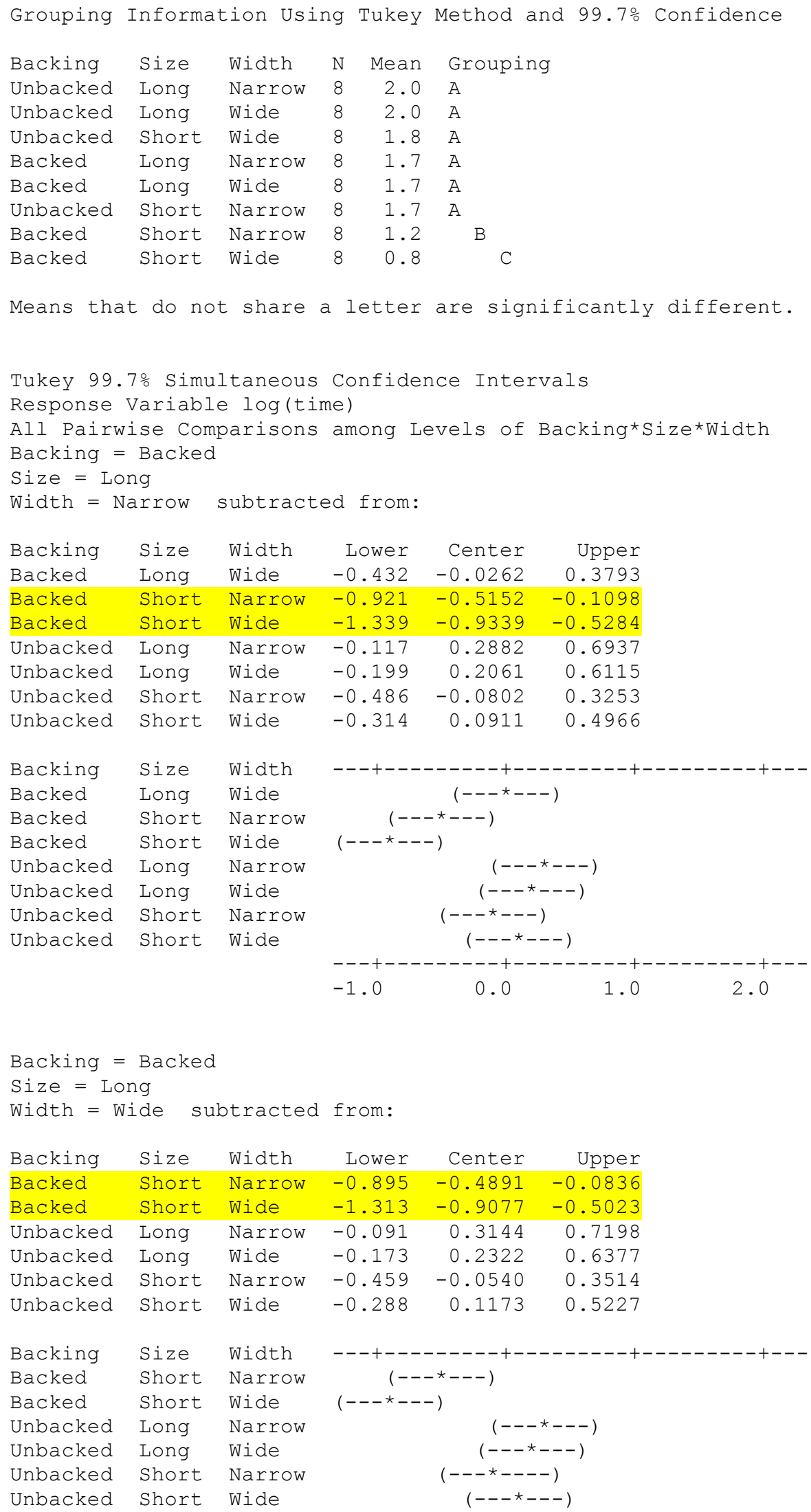




\begin{tabular}{|c|}
\hline-1.0 \\
\hline
\end{tabular}

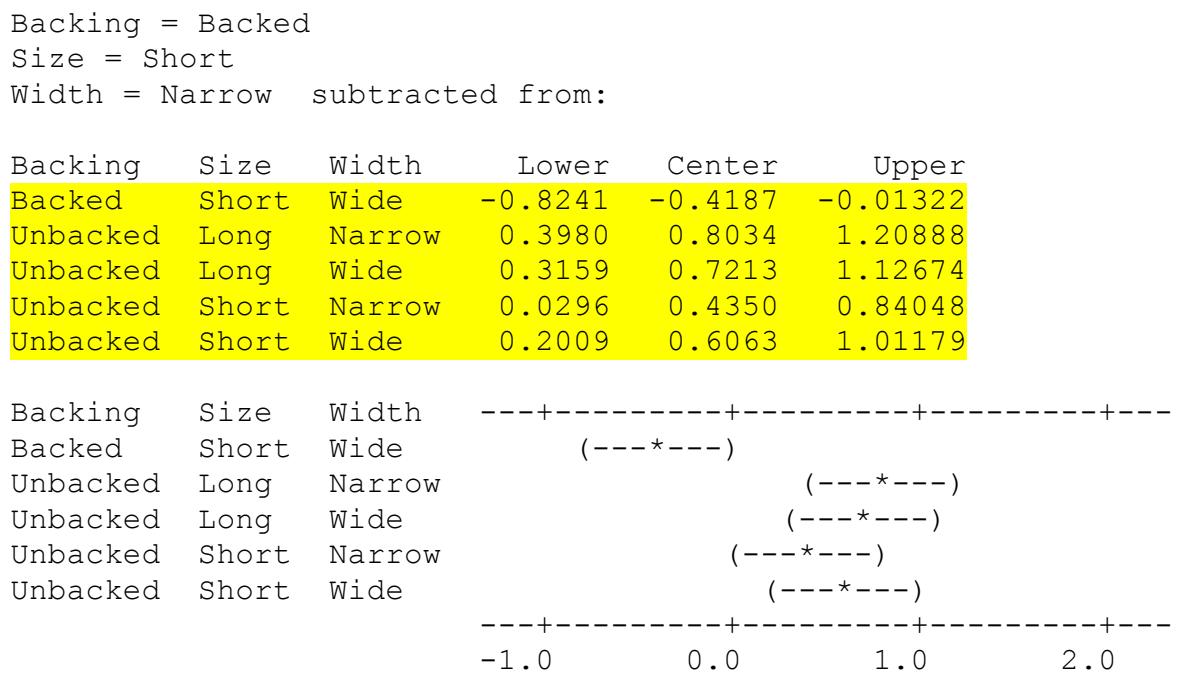

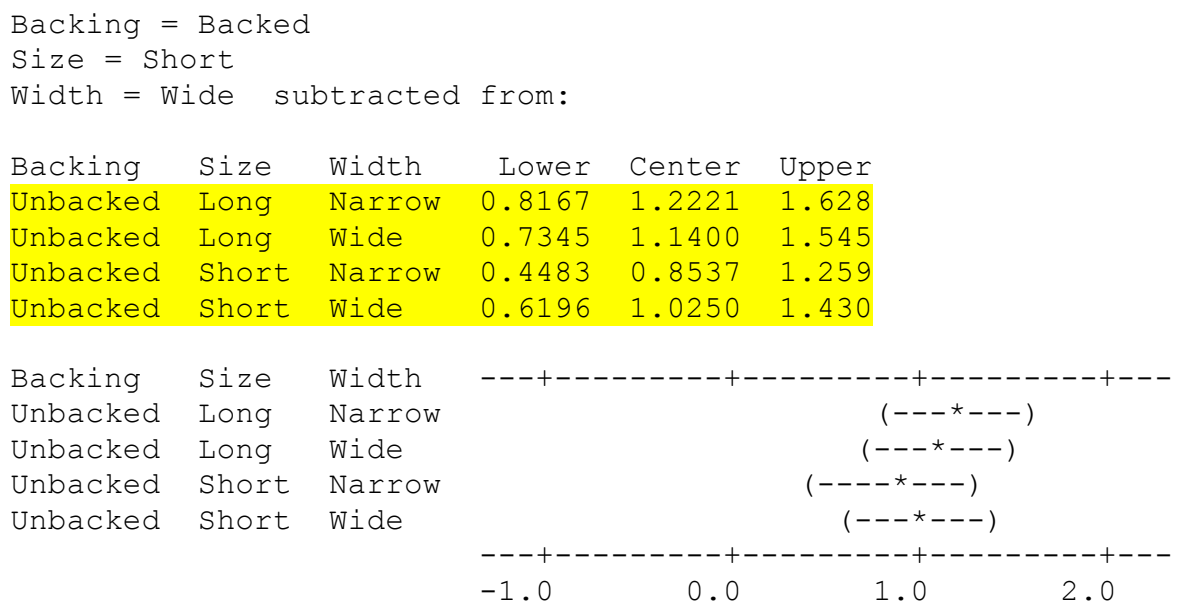

Backing = Unbacked

Size = Long

Width $=$ Narrow subtracted from:

Backing Size Width Lower Center Upper

$\begin{array}{lllll}\text { Unbacked Long Wide } & -0.4876 & -0.0821 & 0.32331\end{array}$

Unbacked Short Narrow $\quad-0.7738 \quad-0.3684 \quad 0.03705$

$\begin{array}{lllll}\text { Unbacked Short Wide } & -0.6025 & -0.1971 & 0.20835\end{array}$

Backing Size Width ---+--------+--------+--------+--

Unbacked Long Wide (---*---)

Unbacked Short Narrow $\quad(---\star---)$

Unbacked Short Wide $(---\star---)$
$-1.0$
0.0
1.0
2.0

Backing $=$ Unbacked

Size = Long 


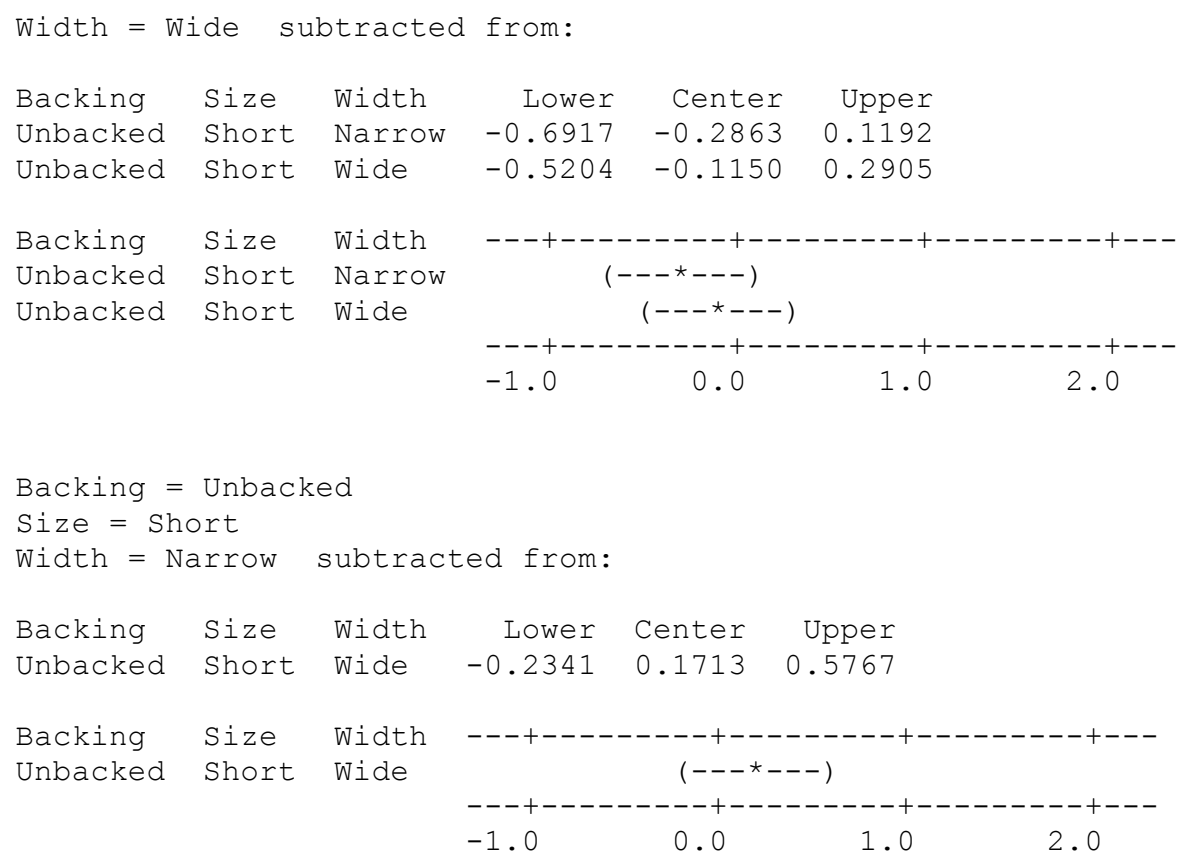




\section{Appendix B: Three-Dimensional AutoCAD Chip Drawings and Designs}

The engineering designs for the three-dimensional chip iterations are presented in this section. The initial set of designs will apply to the first iteration of the designed chip. Following will be the altered base chip for the second design iteration. After, is the altered final layer of the third design iteration. Then, the designs for the final design iteration are presented.

These chip designs are followed by the design that was used in the statistical analyses of wicking time. Likewise, the designs that looked at wicking time with and without a conjugate pad follow. Finally, this is followed by the conceptual SolidWorks engineering drawings for the manufacturing tool to be rapid prototyped and used to piece together the multi-layer chip.

Finally presented are a series of conceptual designs that could be used in the future. The detection regions on these chips have different shapes in order for the user to distinguish the result and the analyte that is detected between each zone. 


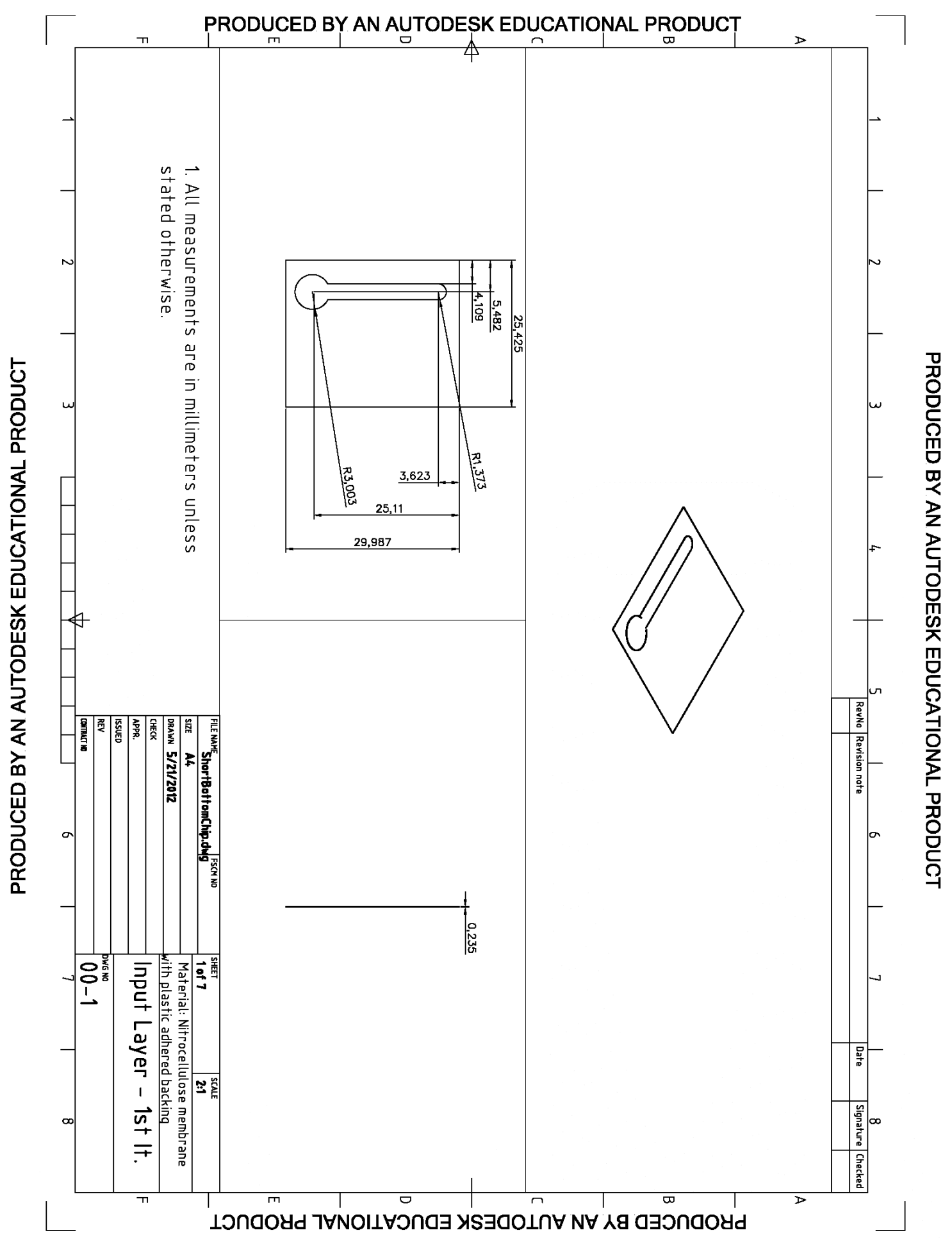




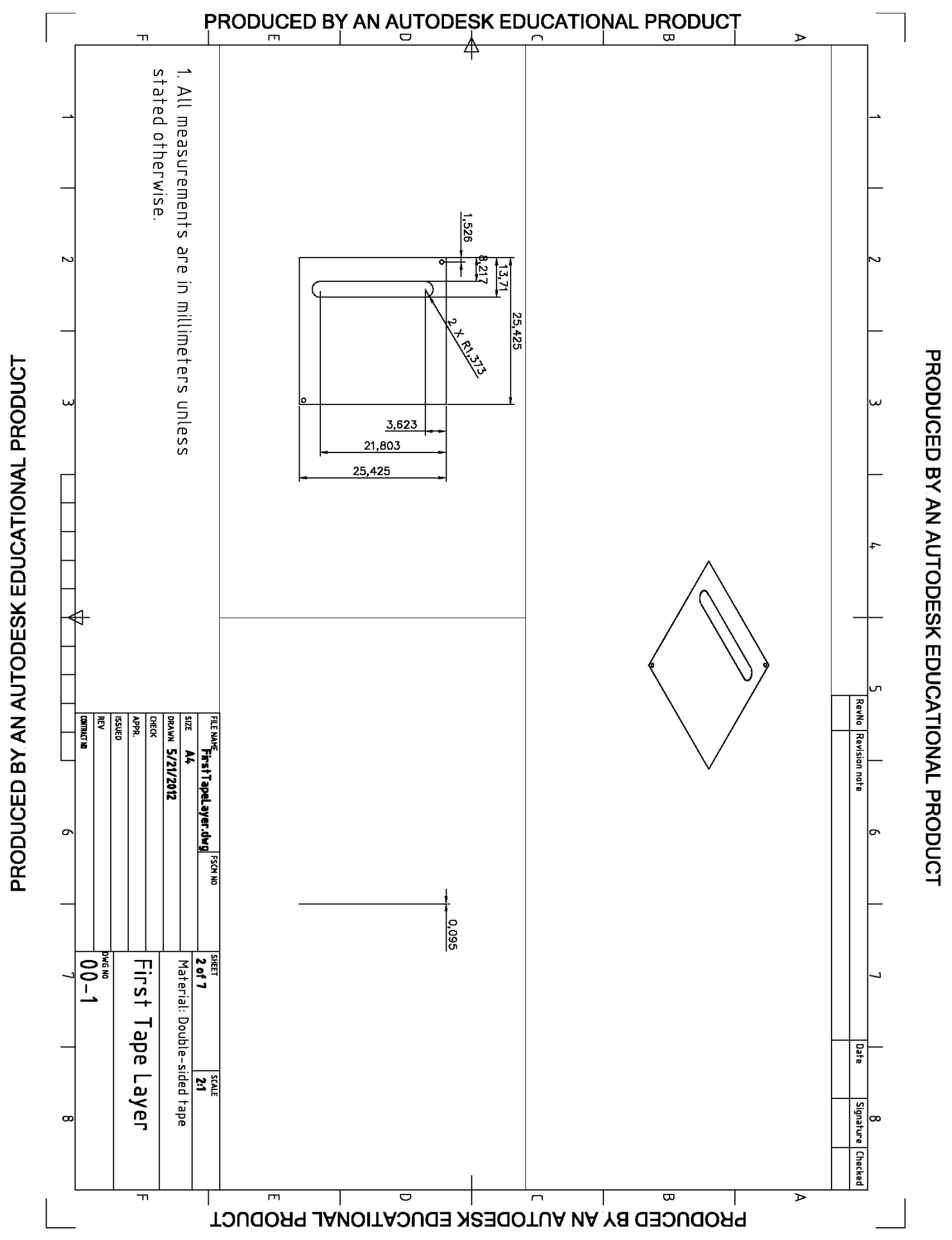




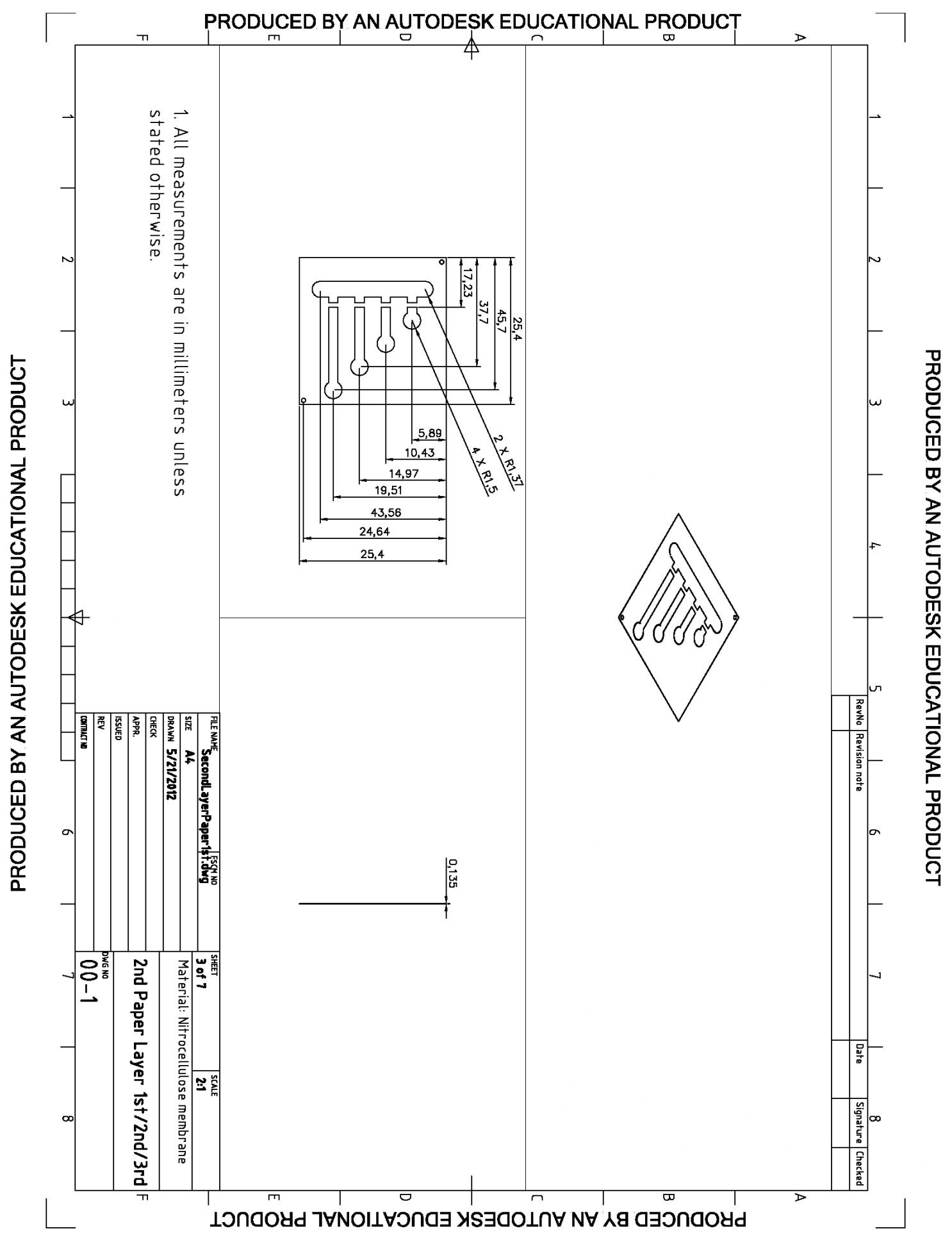




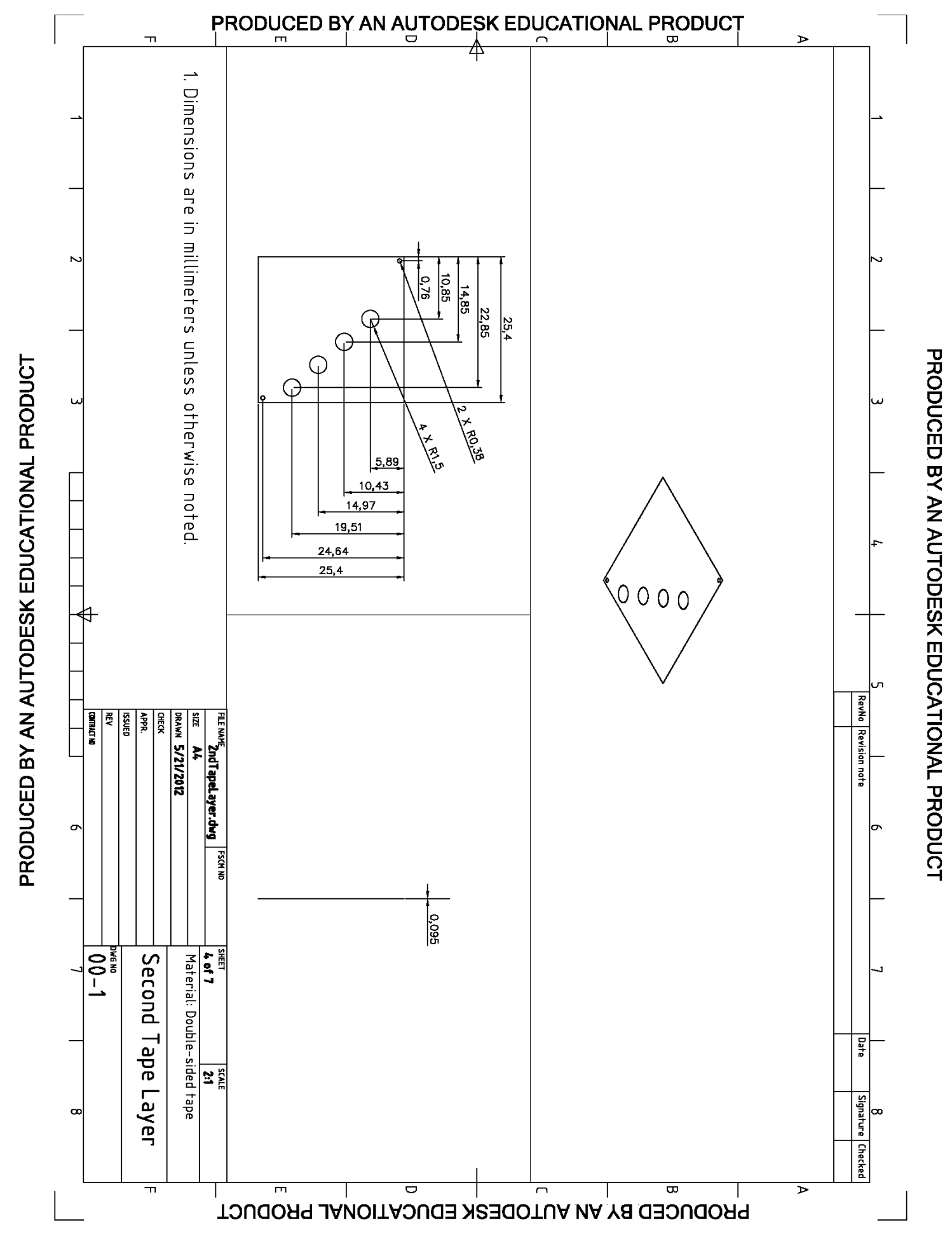




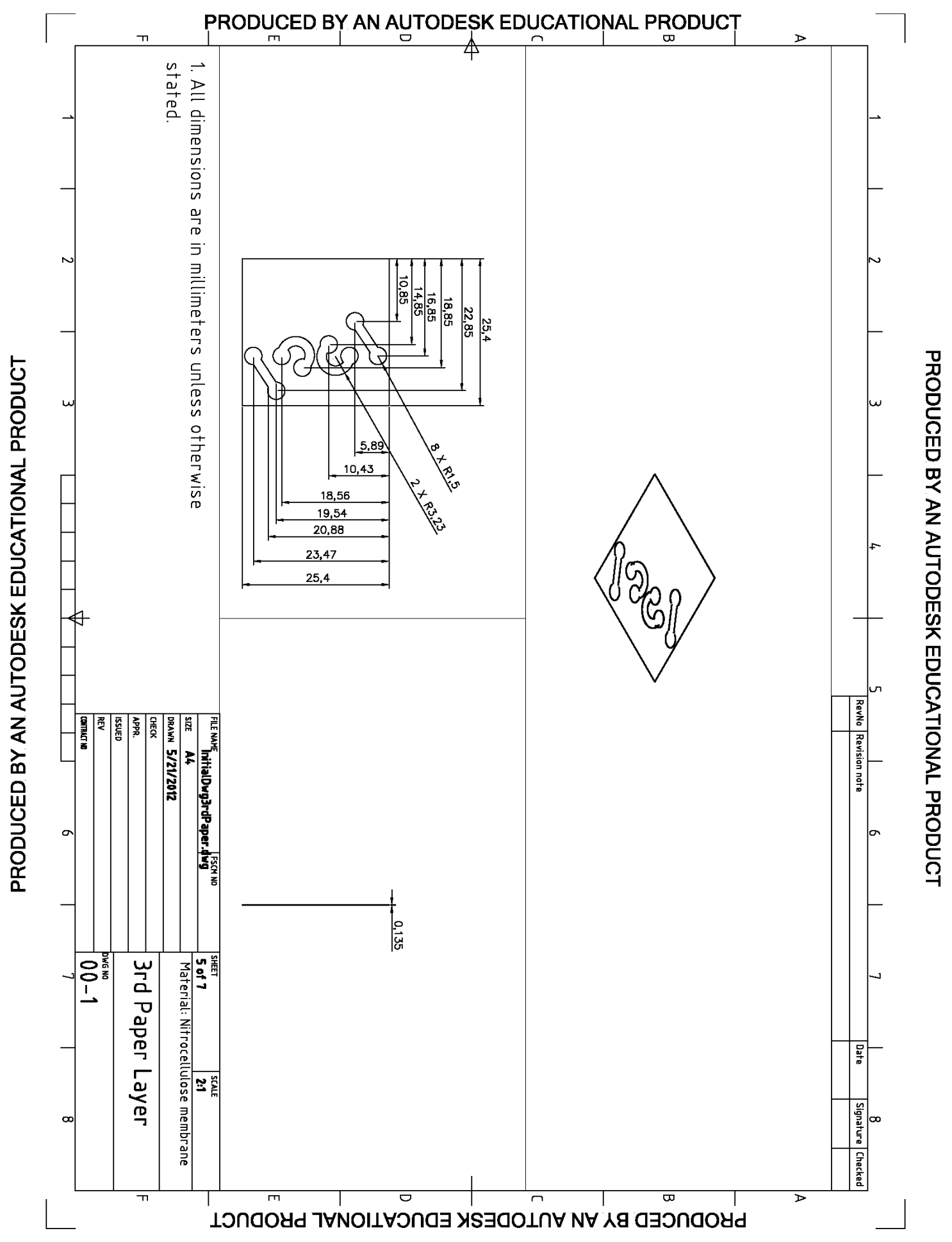




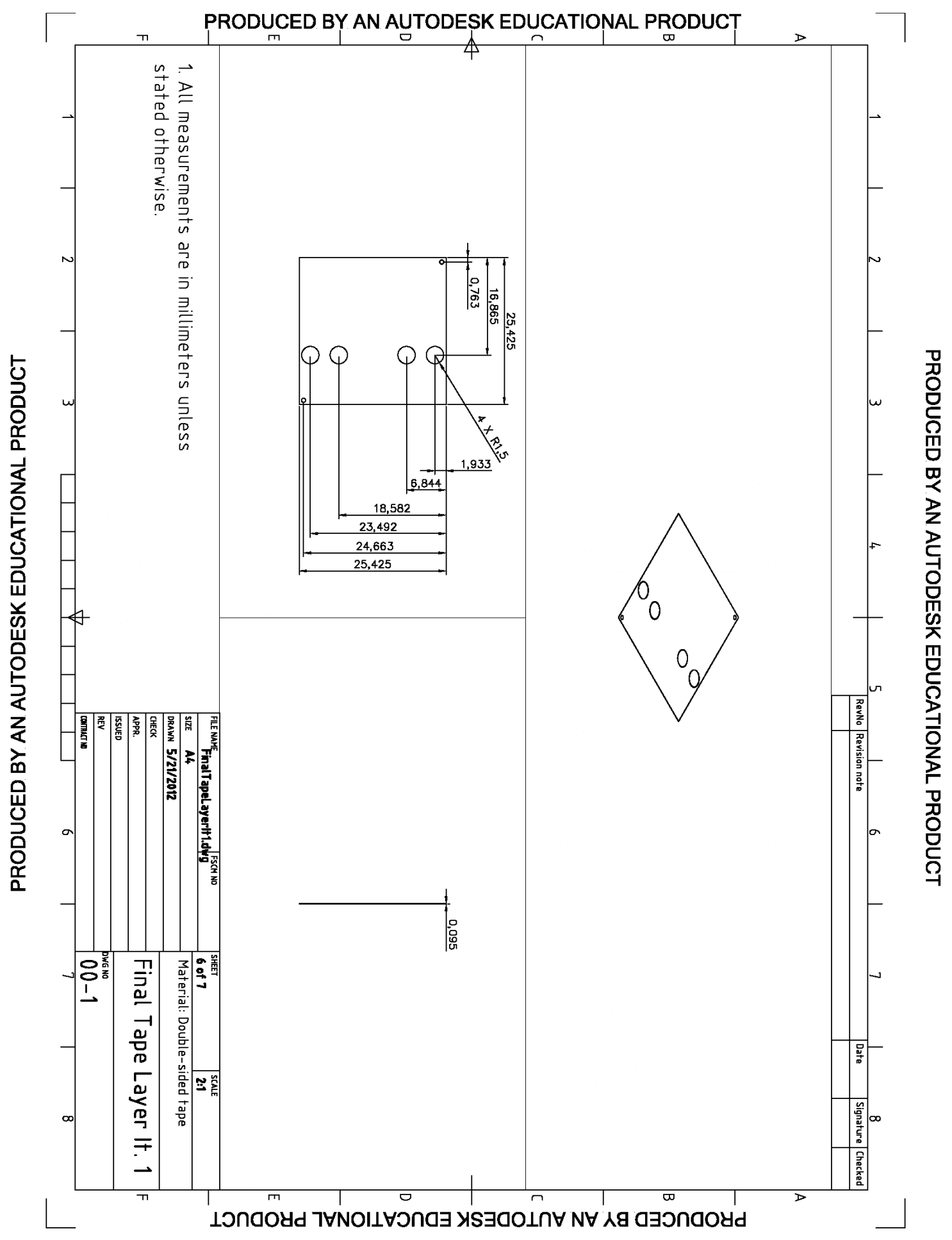




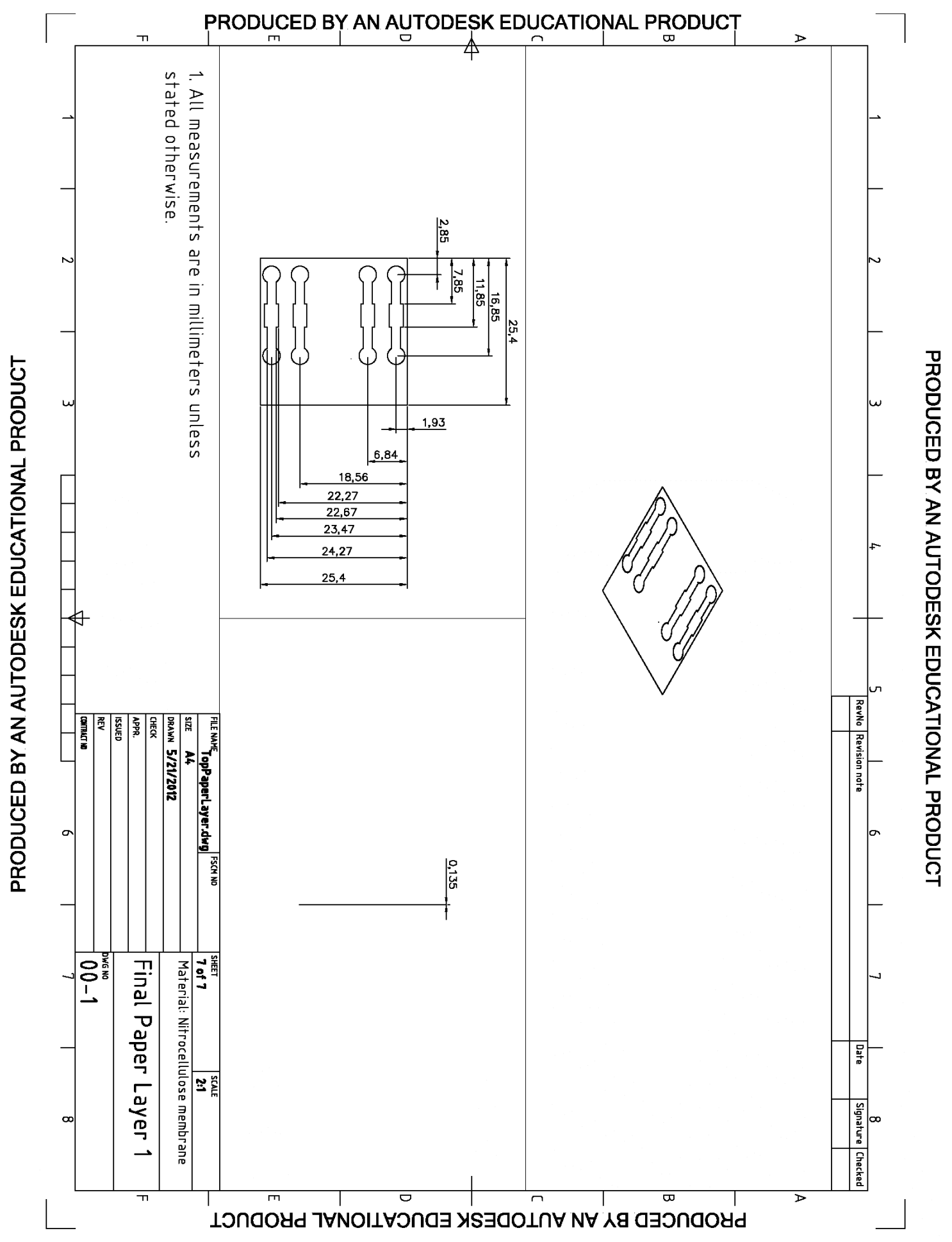




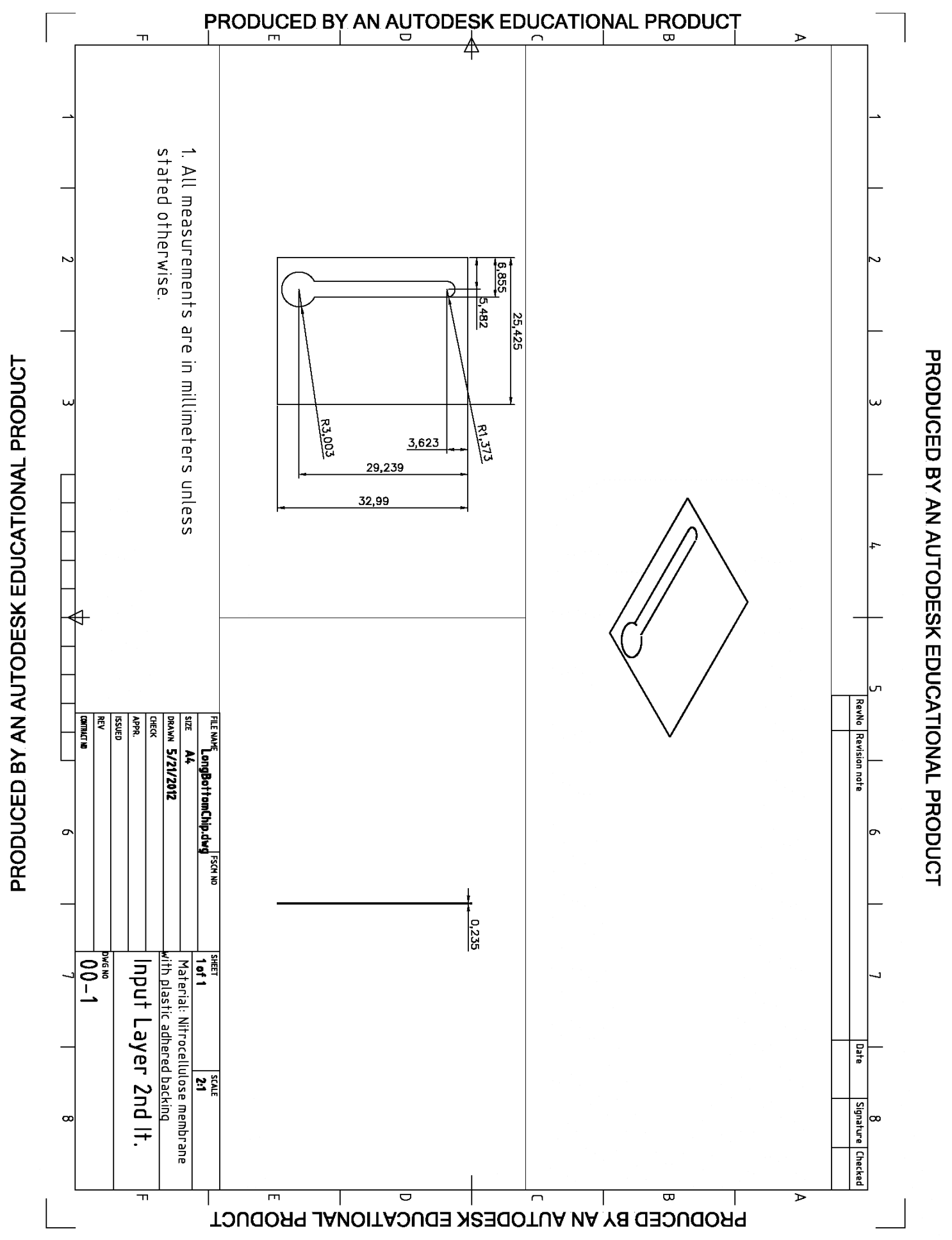




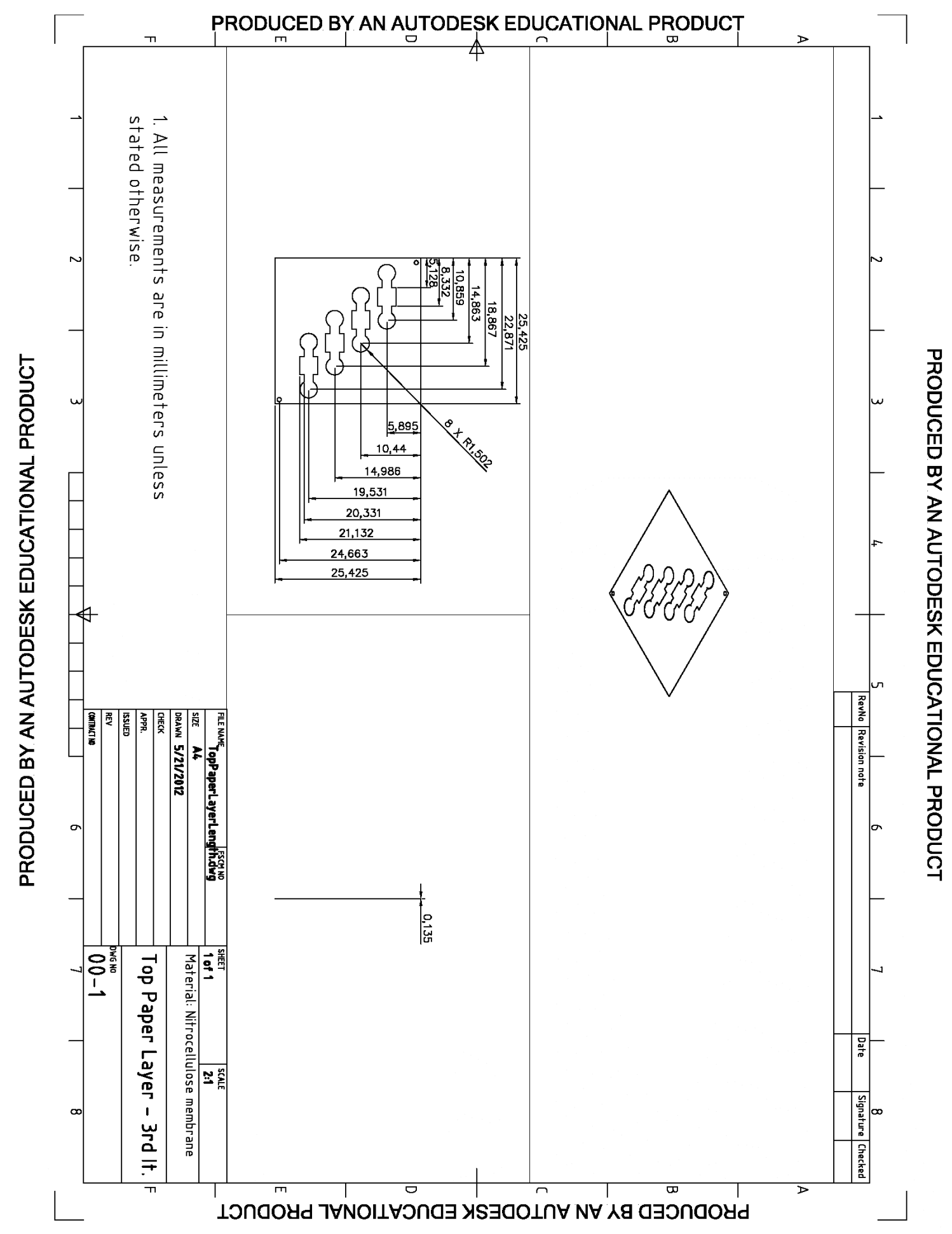




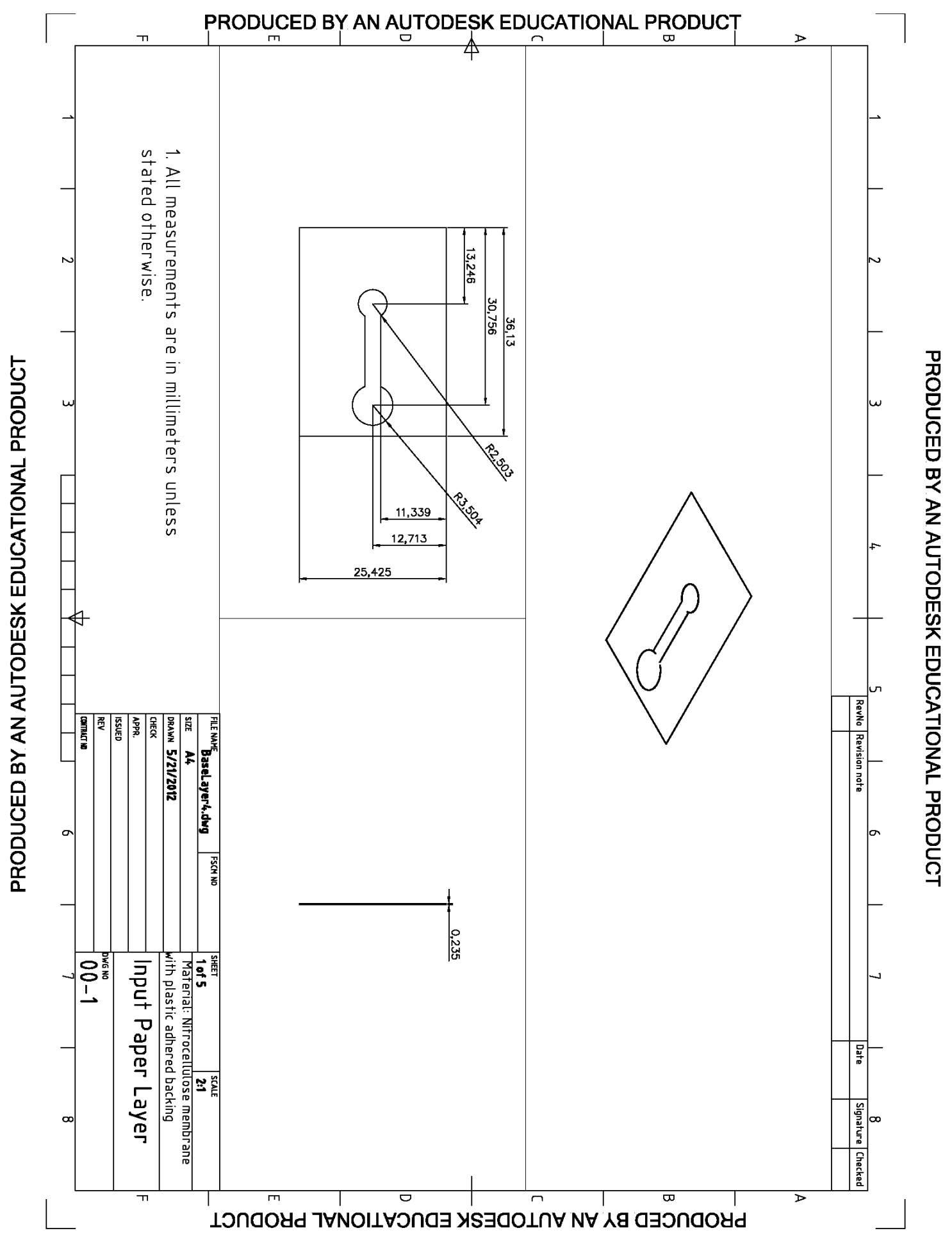




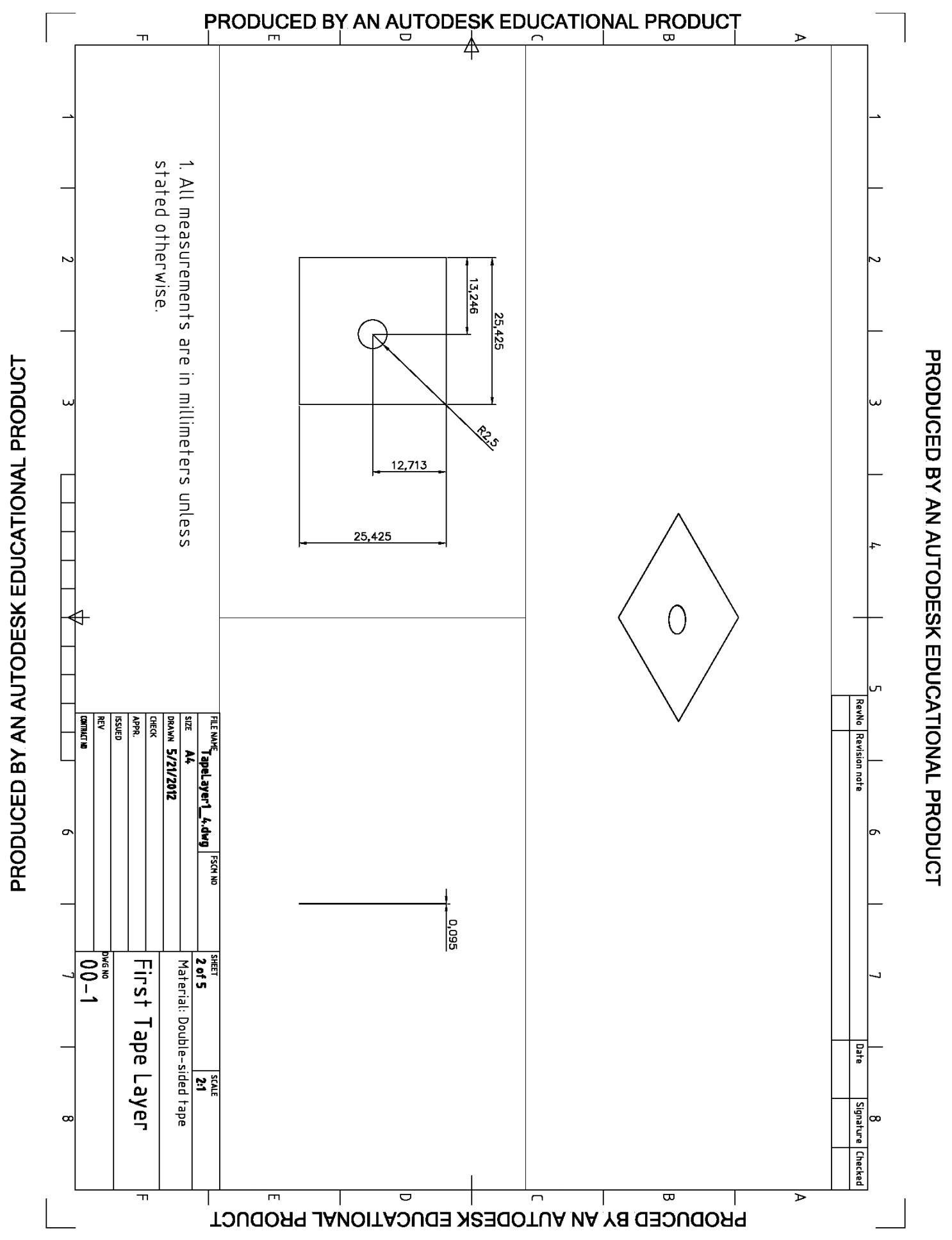




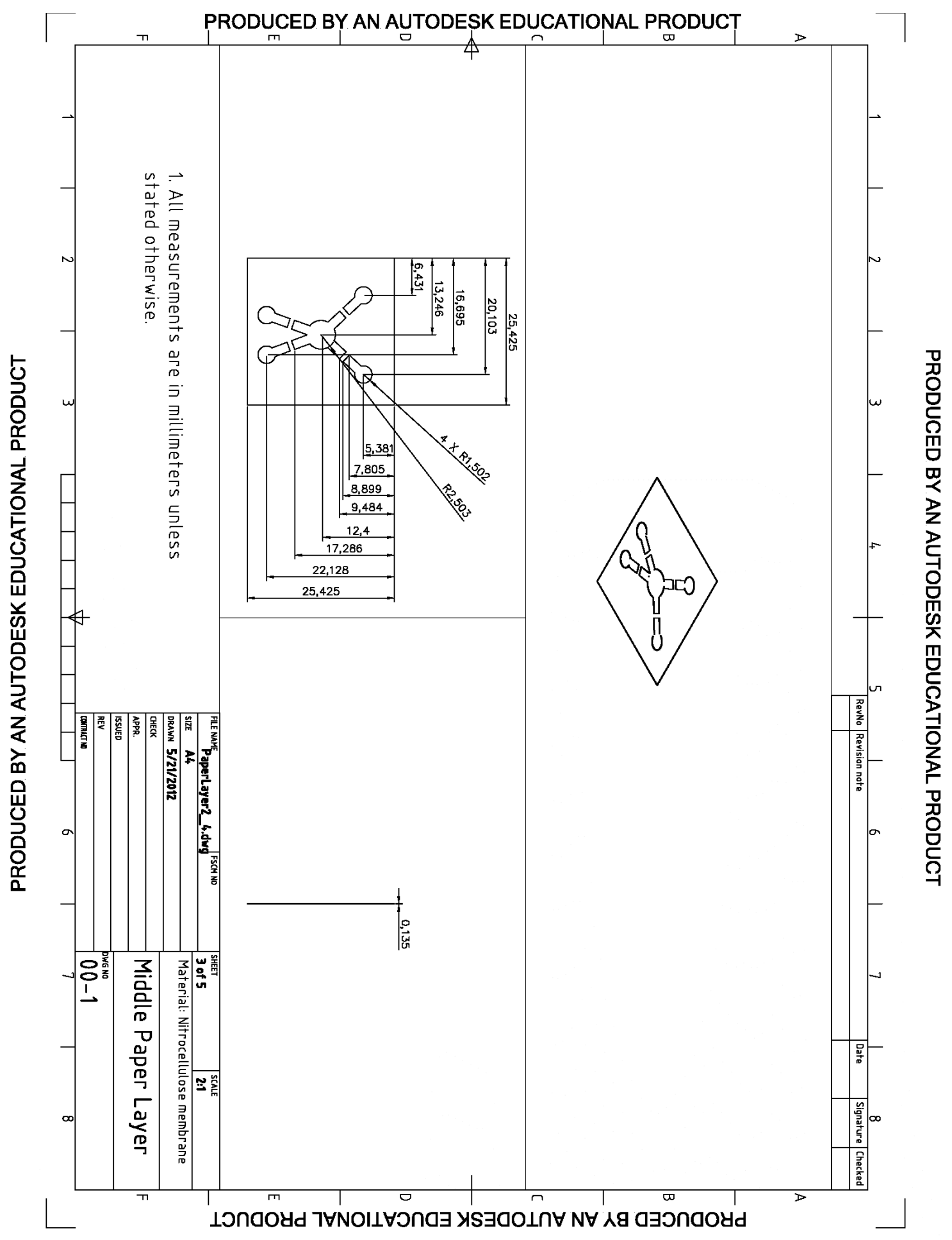




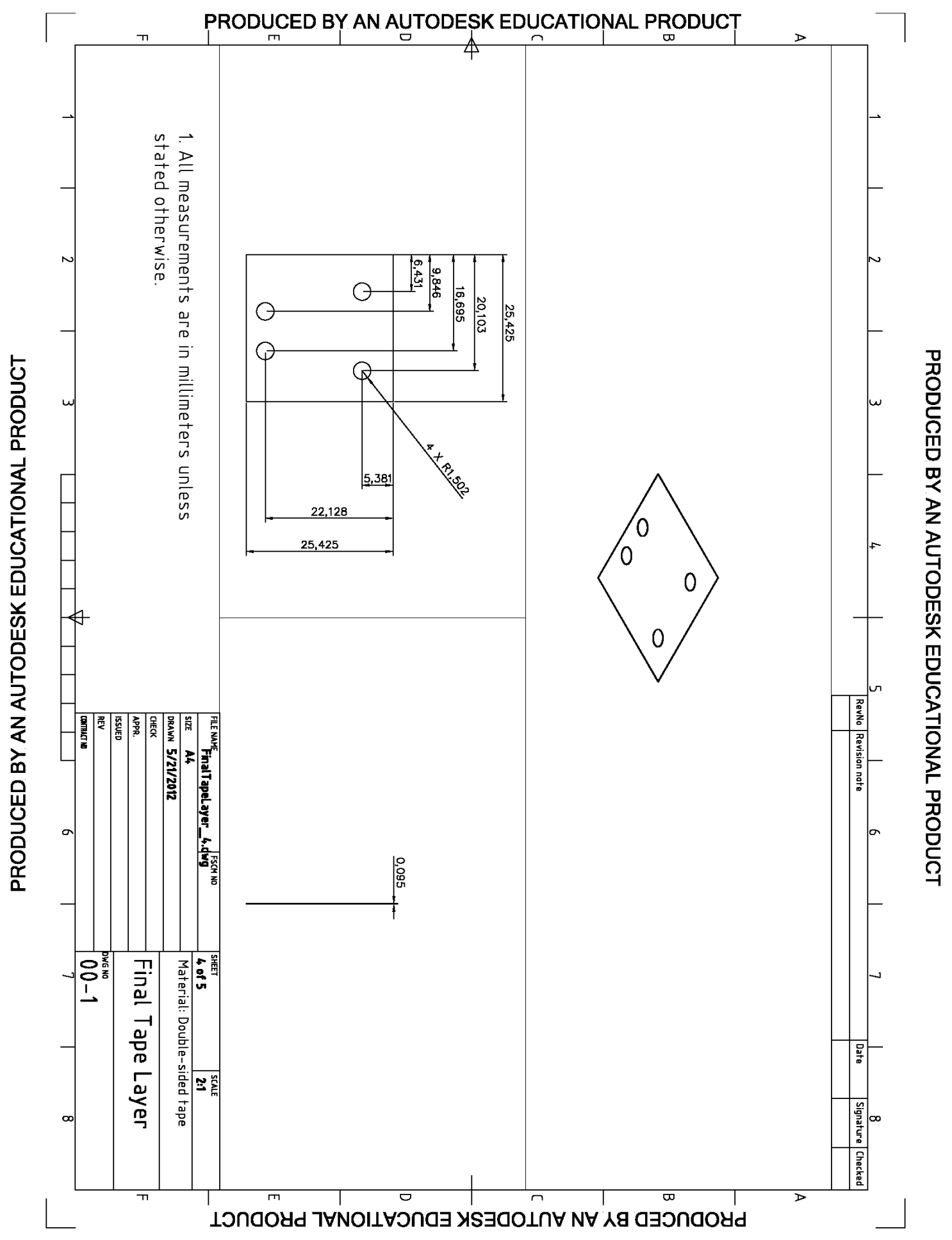




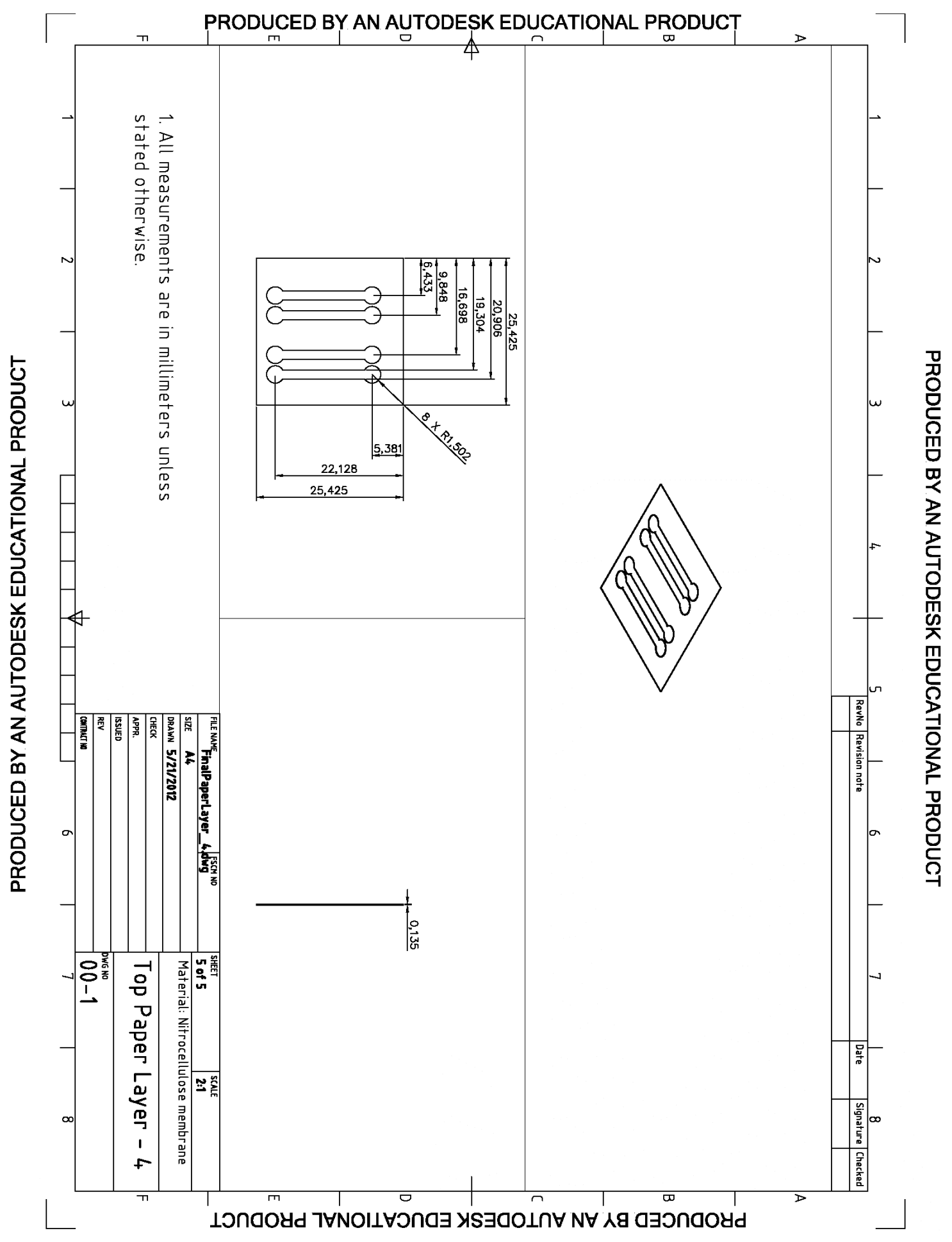




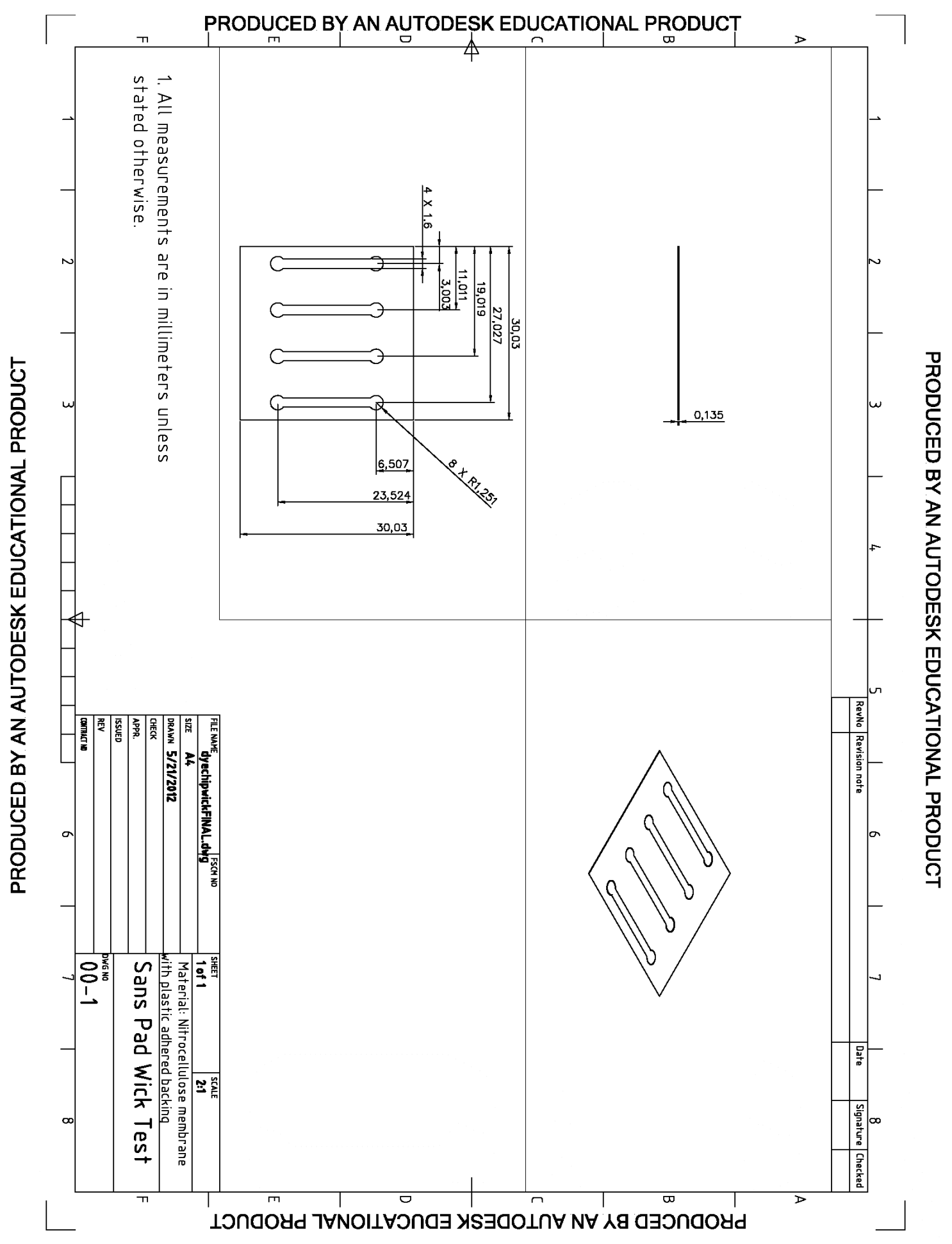




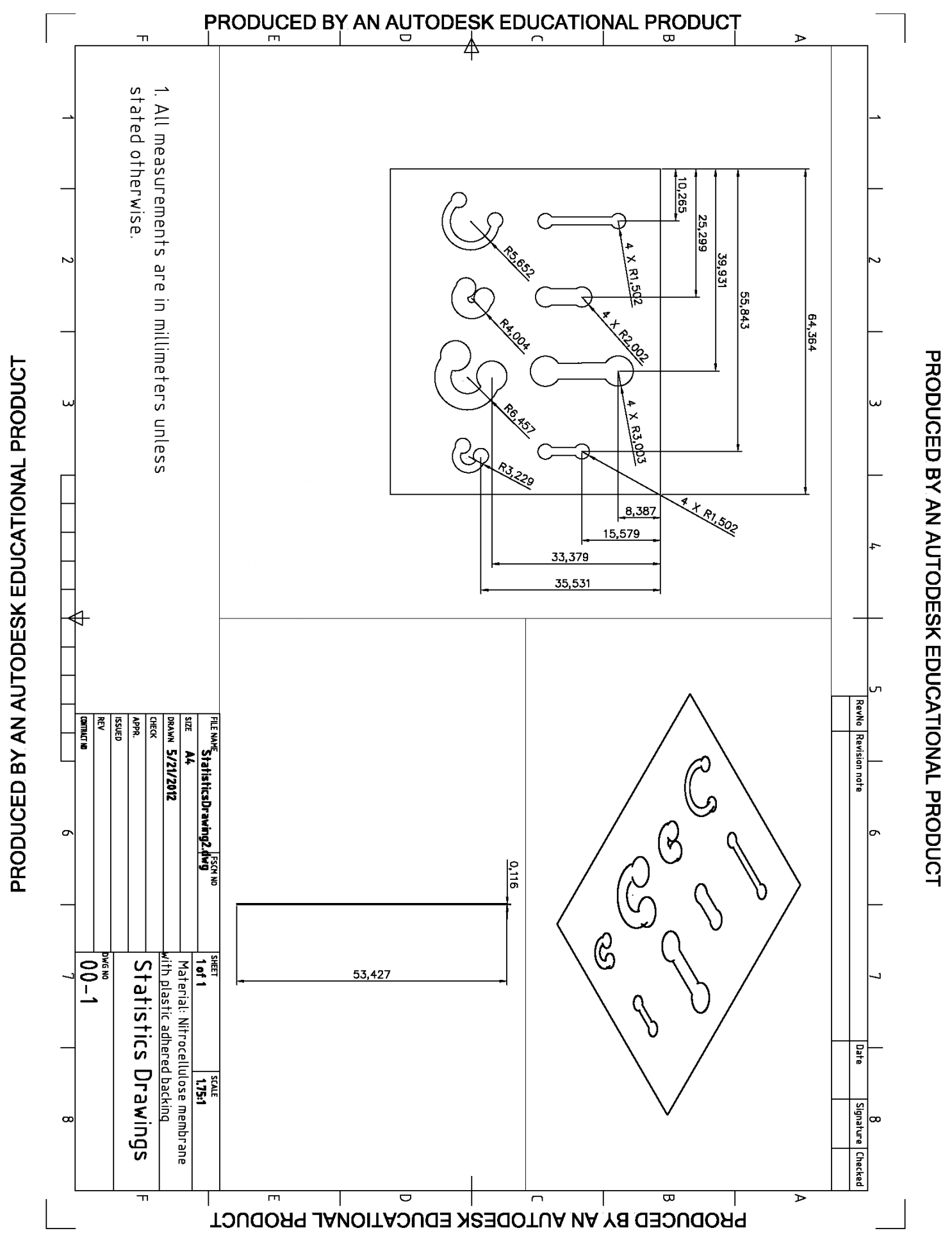




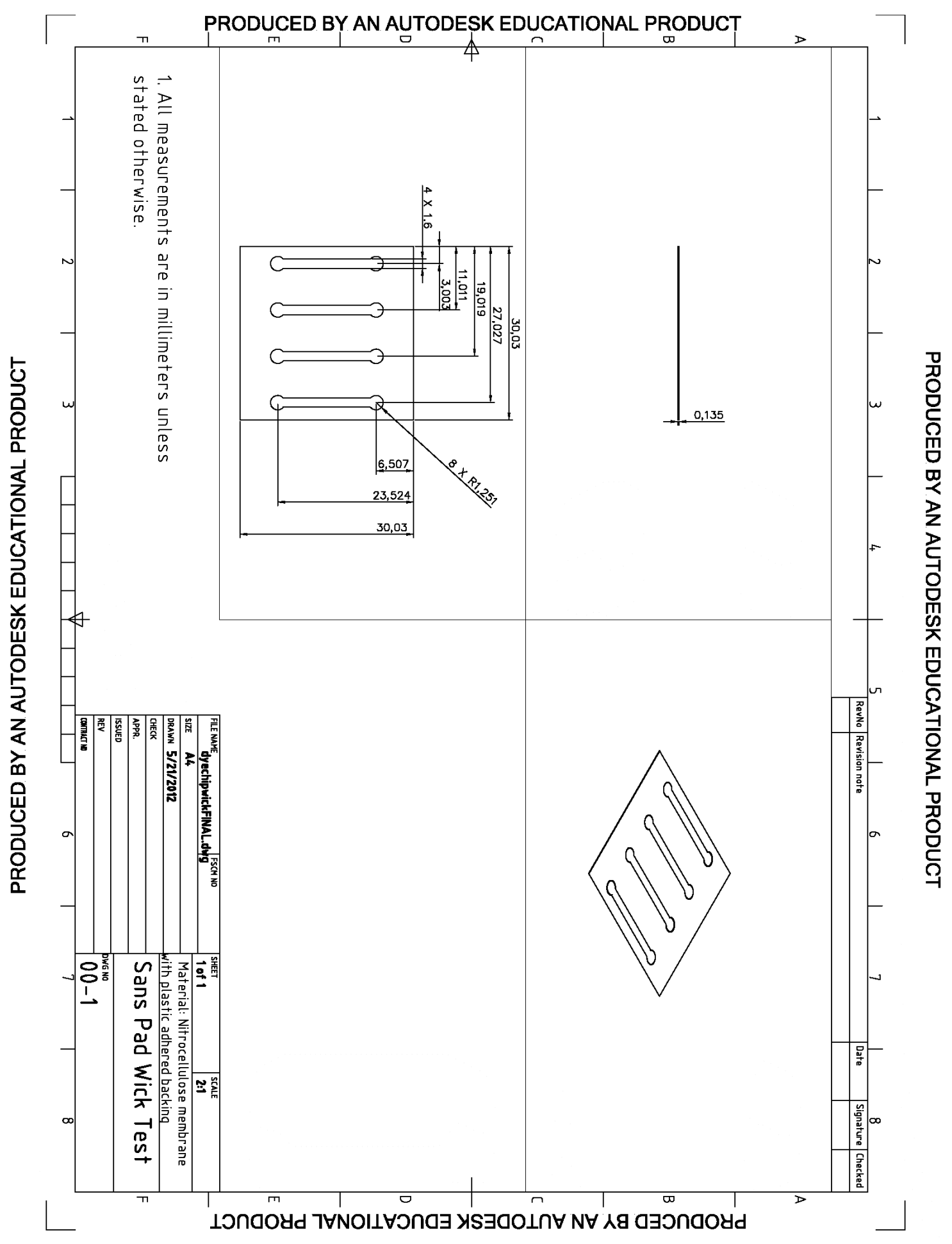




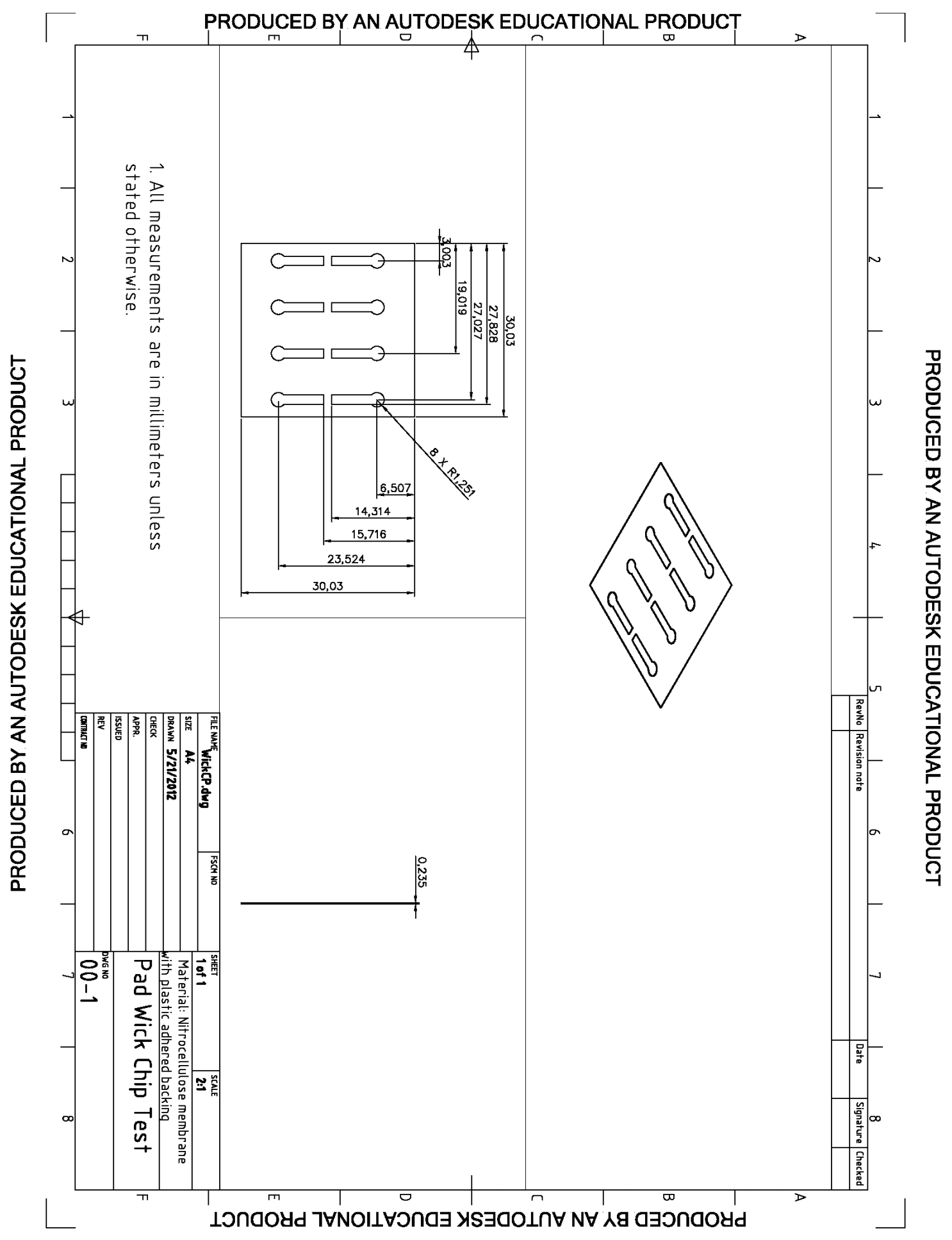




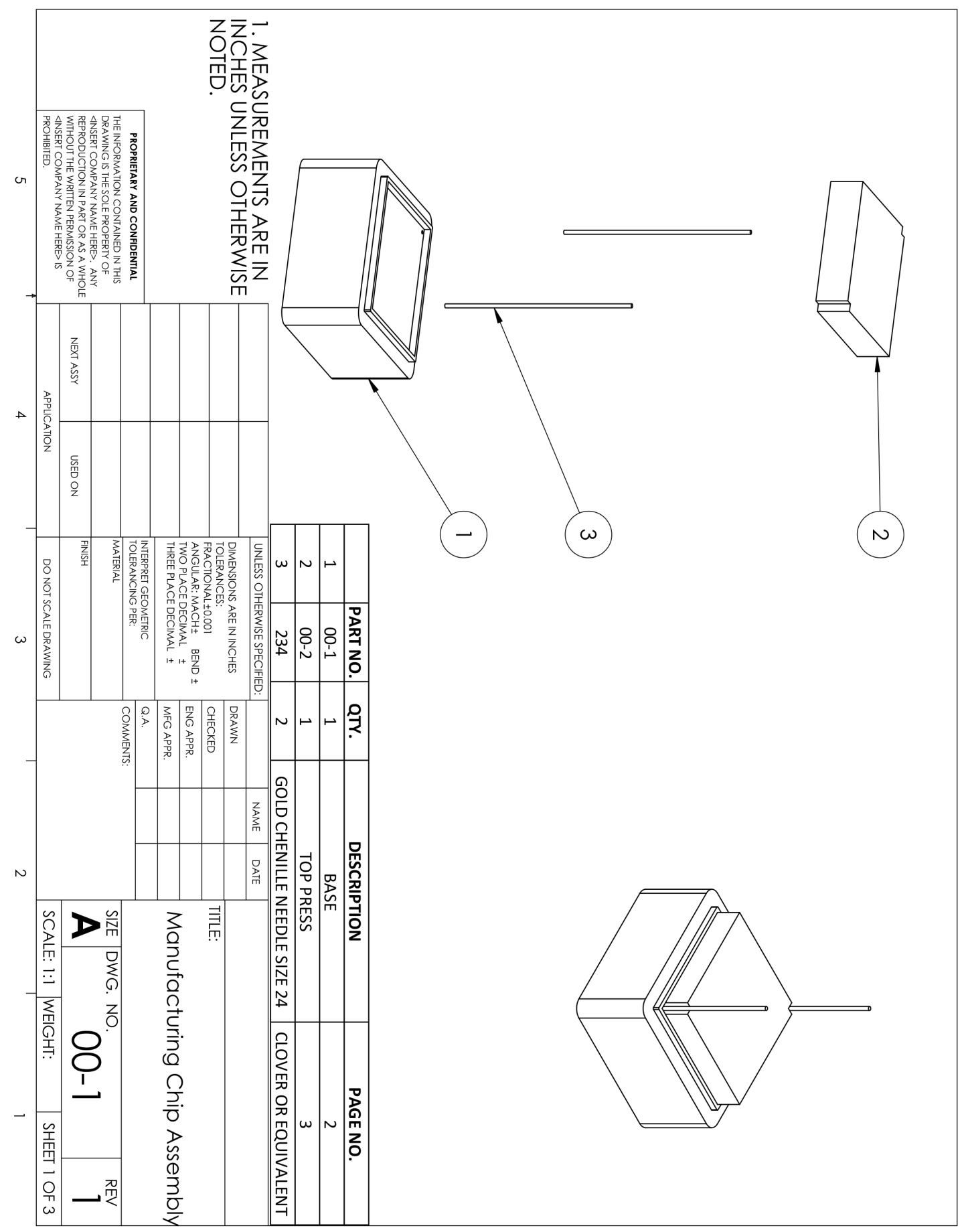




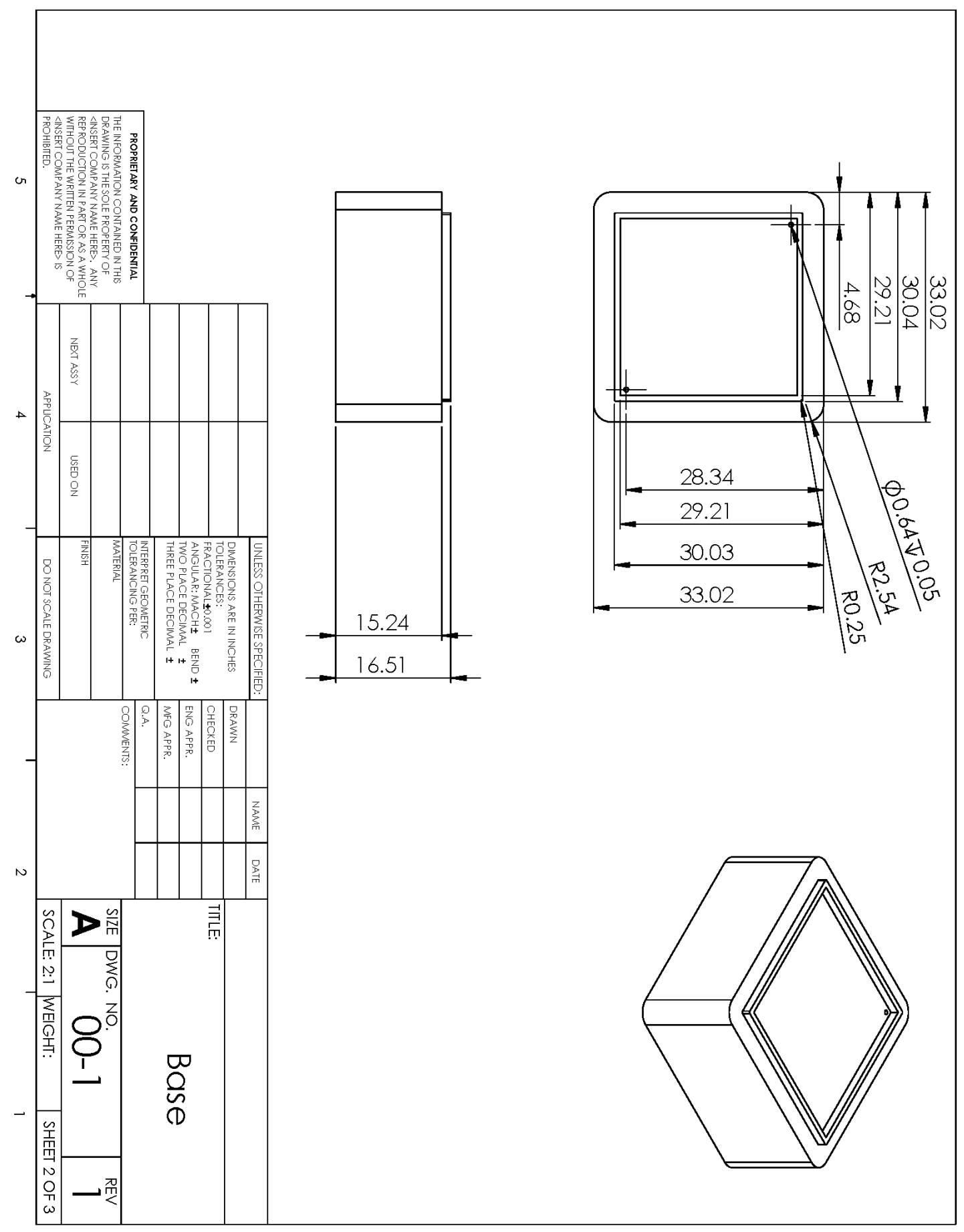




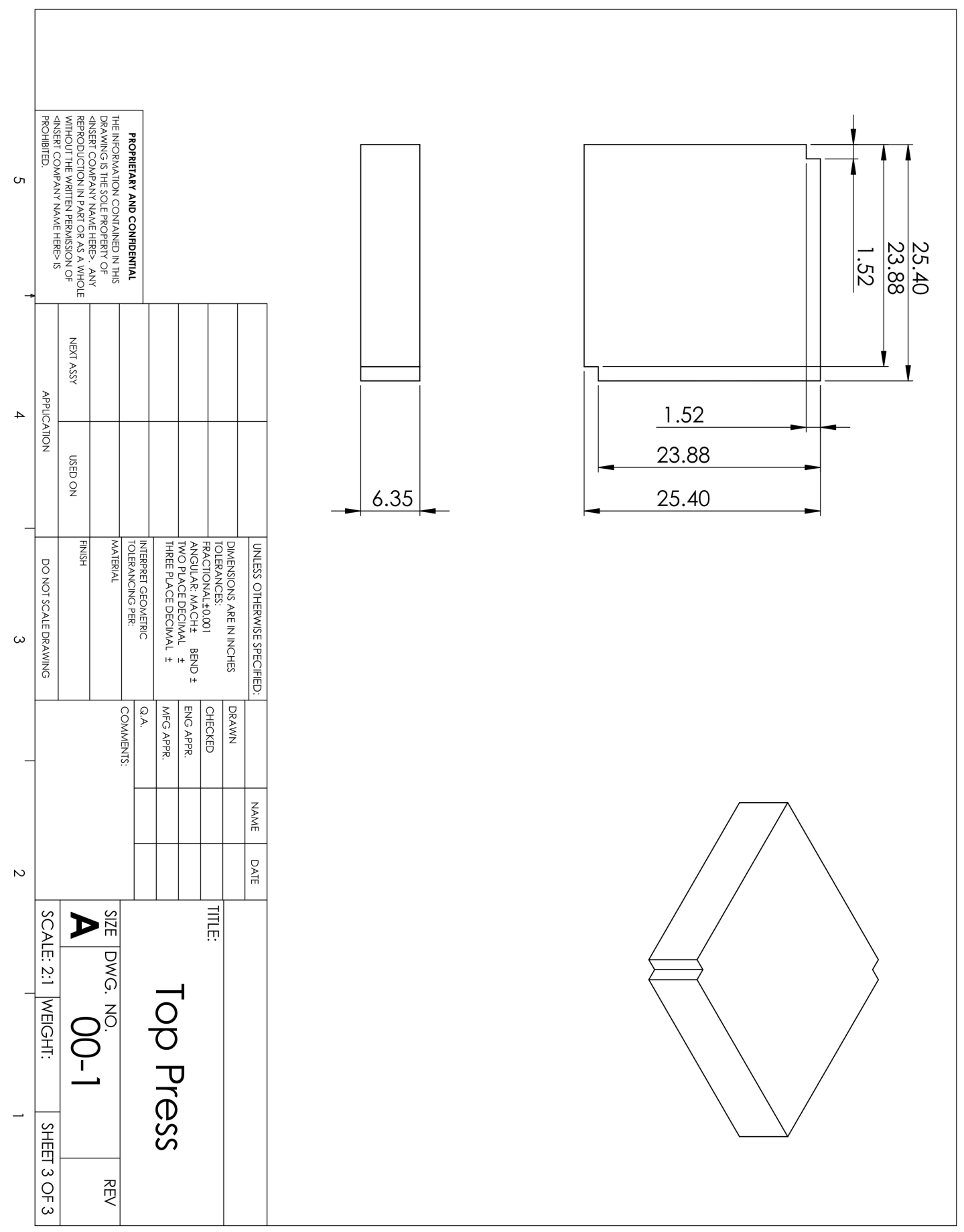




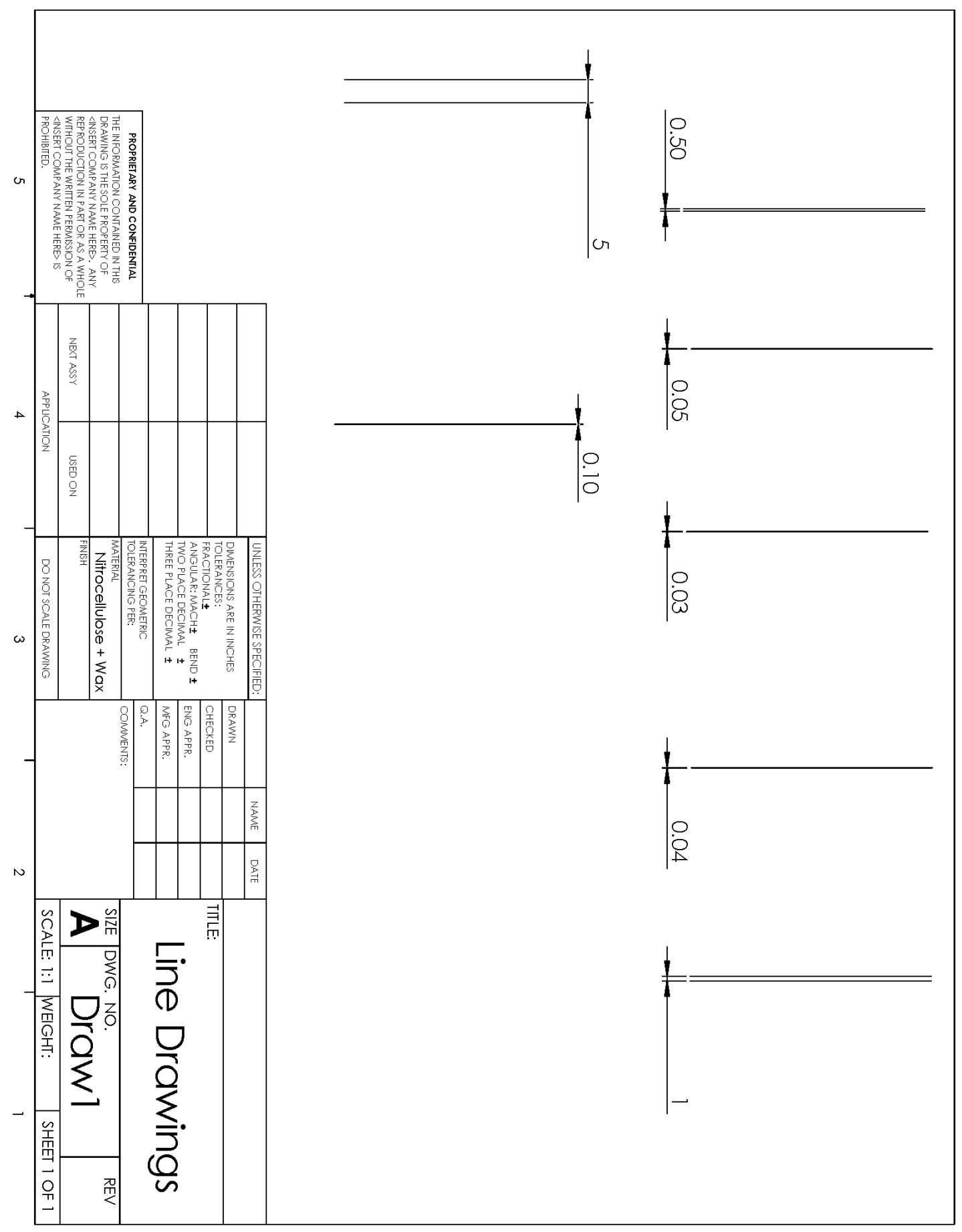

Note: All measurements are in millimeters unless stated 


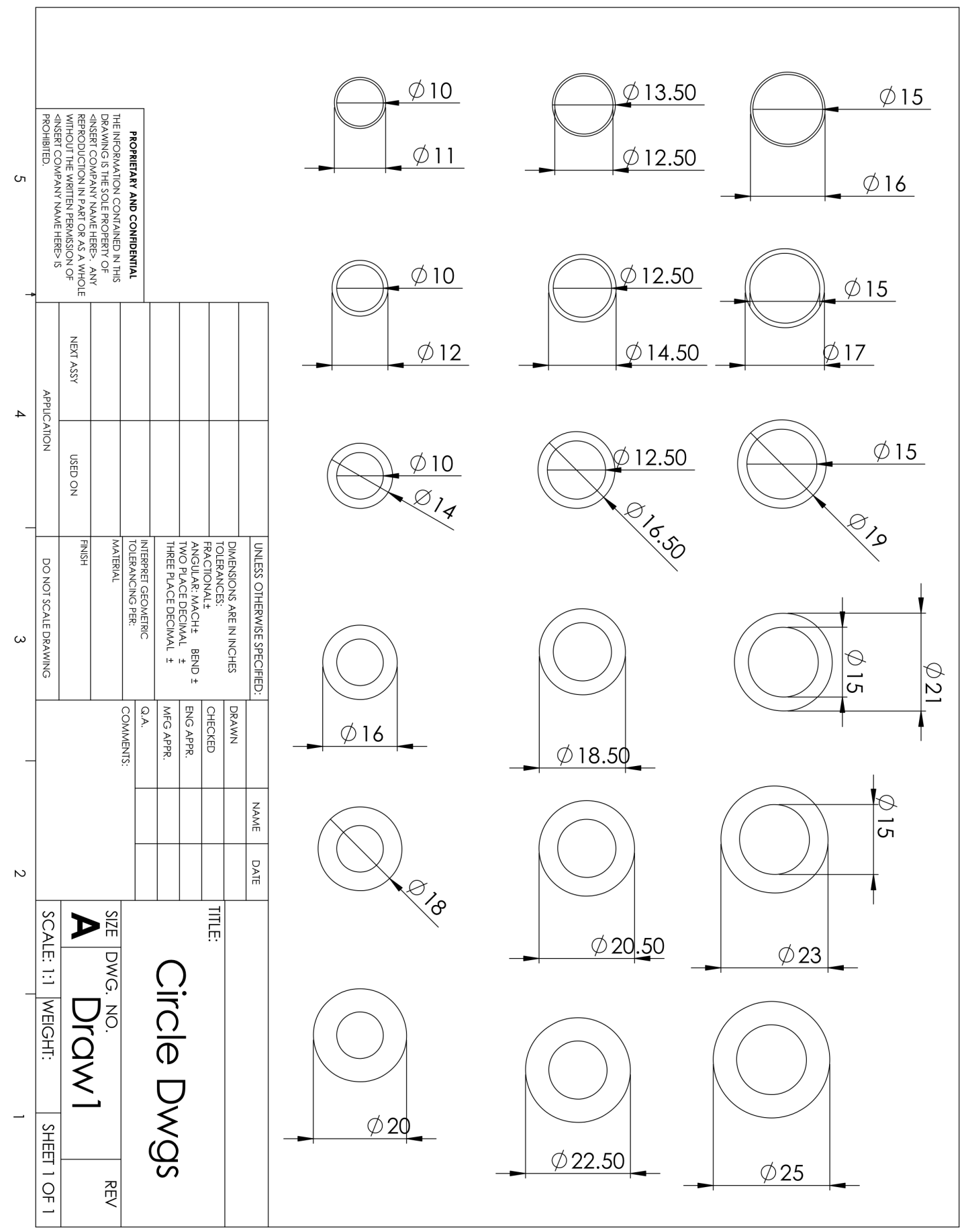

Note: All measurements are in millimeters unless stated 


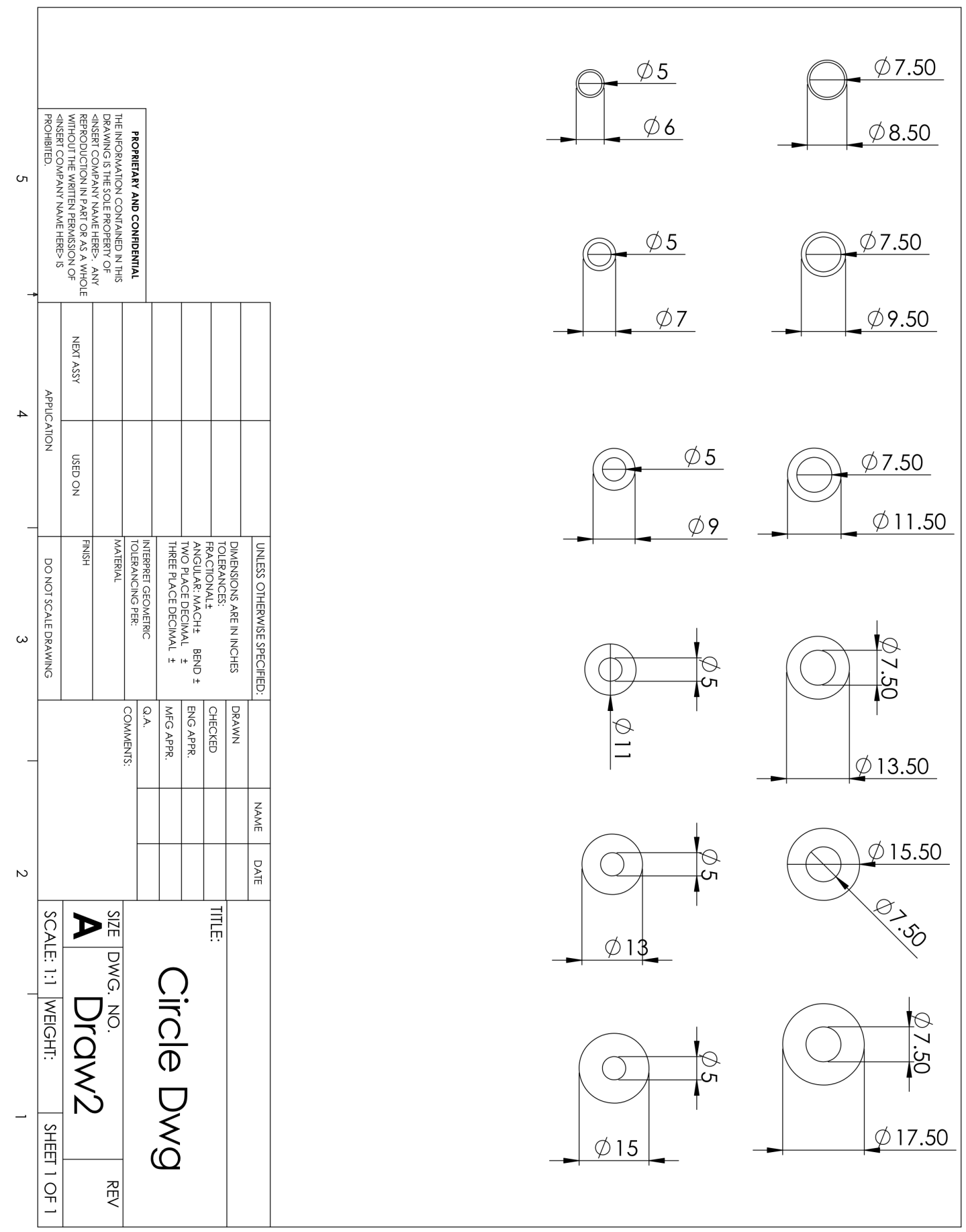

Note: All measurements are in millimeters unless stated 


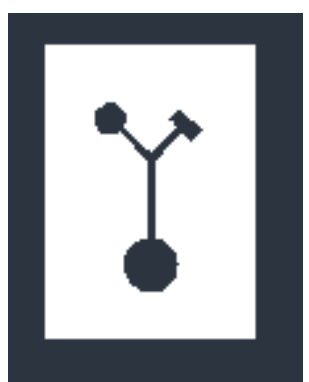

Figure 40: 2D Two Analyte Detection Design. A proposed future design for a $2 \mathrm{D}$, two analyte detection chip. The two detection regions are different shapes for the user to more easily distinguish the end result.

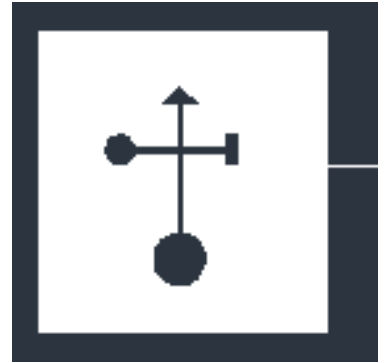

Figure 41: 2D Three Analyte Detection Design. A proposed future design for a $2 \mathrm{D}$, three analyte detection chip. The three detection regions are different shapes for the user to more easily distinguish the end result.

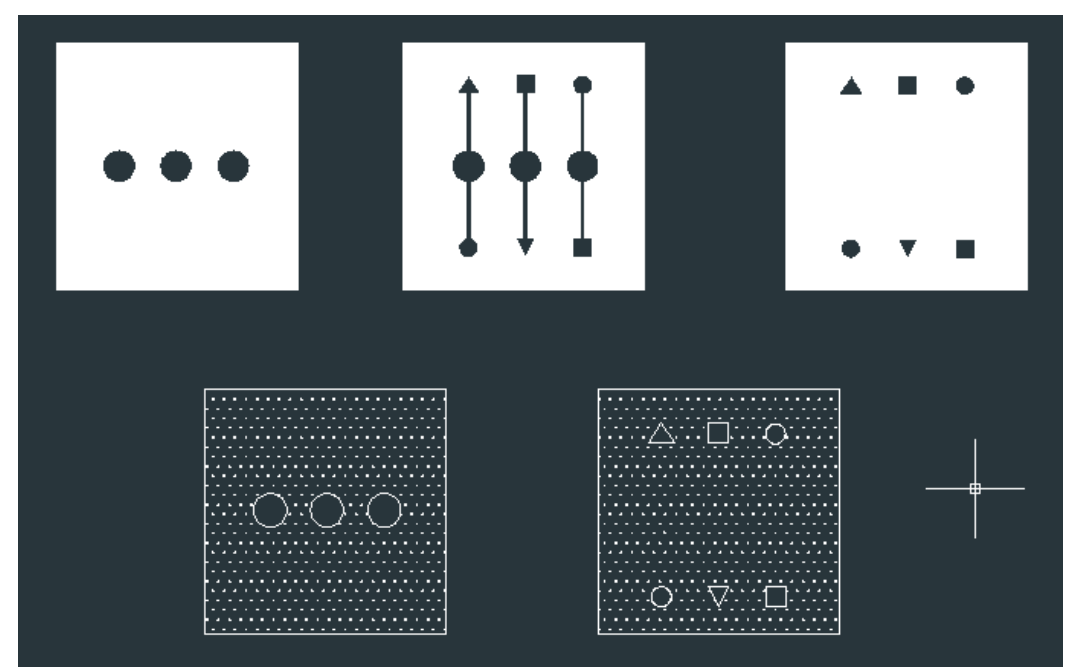

Figure 42: 3D Six Analyte Detection Design. A proposed future design for a six analyte detection chip. The six detection regions are different shapes for the user to more easily distinguish the end result. The input is on the far left to the observation on the far right - solid blocks are paper layers and hatched designs are tape layers. 


\section{Appendix C: Two-Dimensional AutoCAD Chip Drawings and Designs}

This section presents the various two-dimensional chip designs that were used to test the antibody and aptamer capture reagents in detecting VEGF in sample.

Following are the laser cut transparency drawings. Then, this is followed by the design used for the colloidal gold visual detection test. 


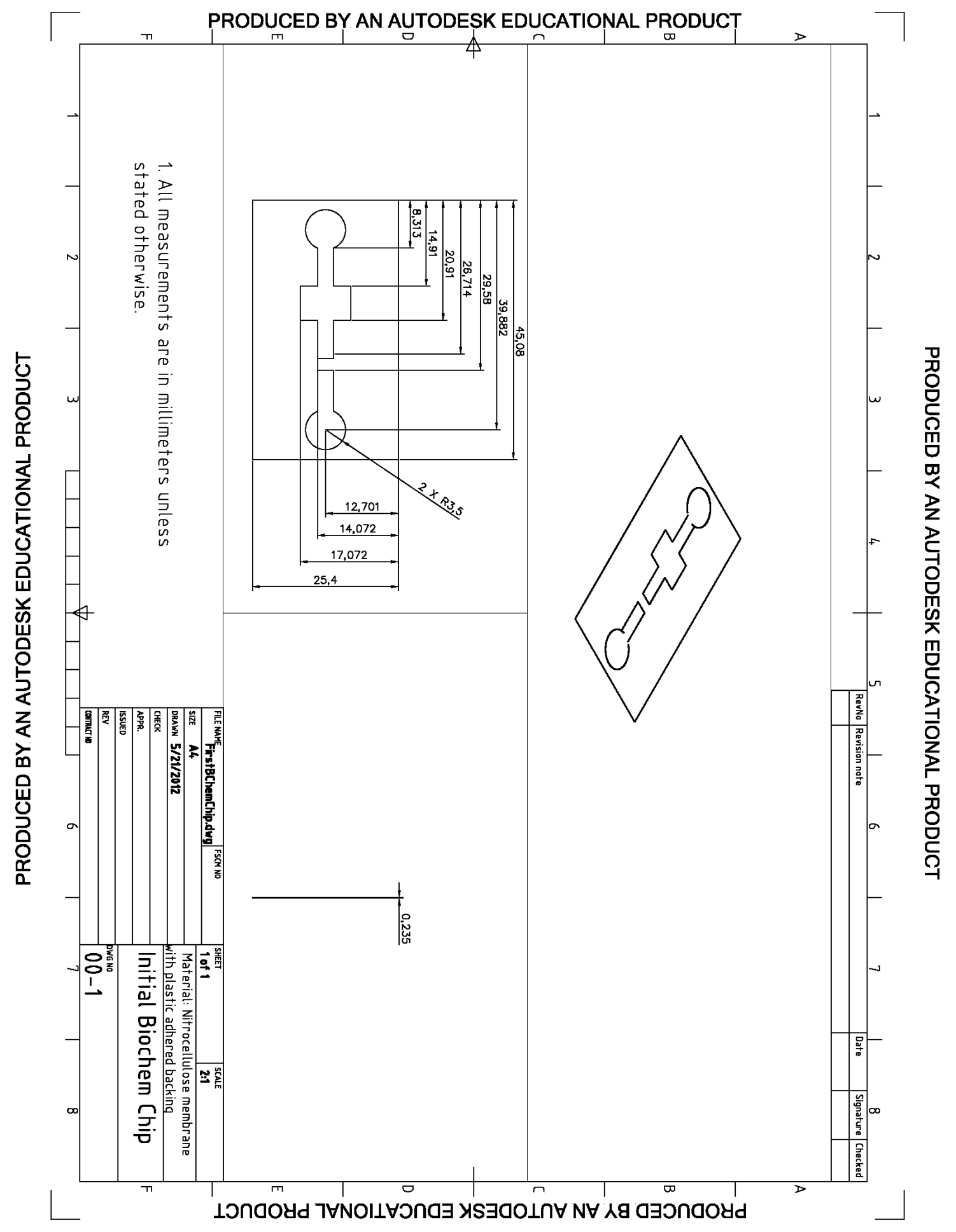




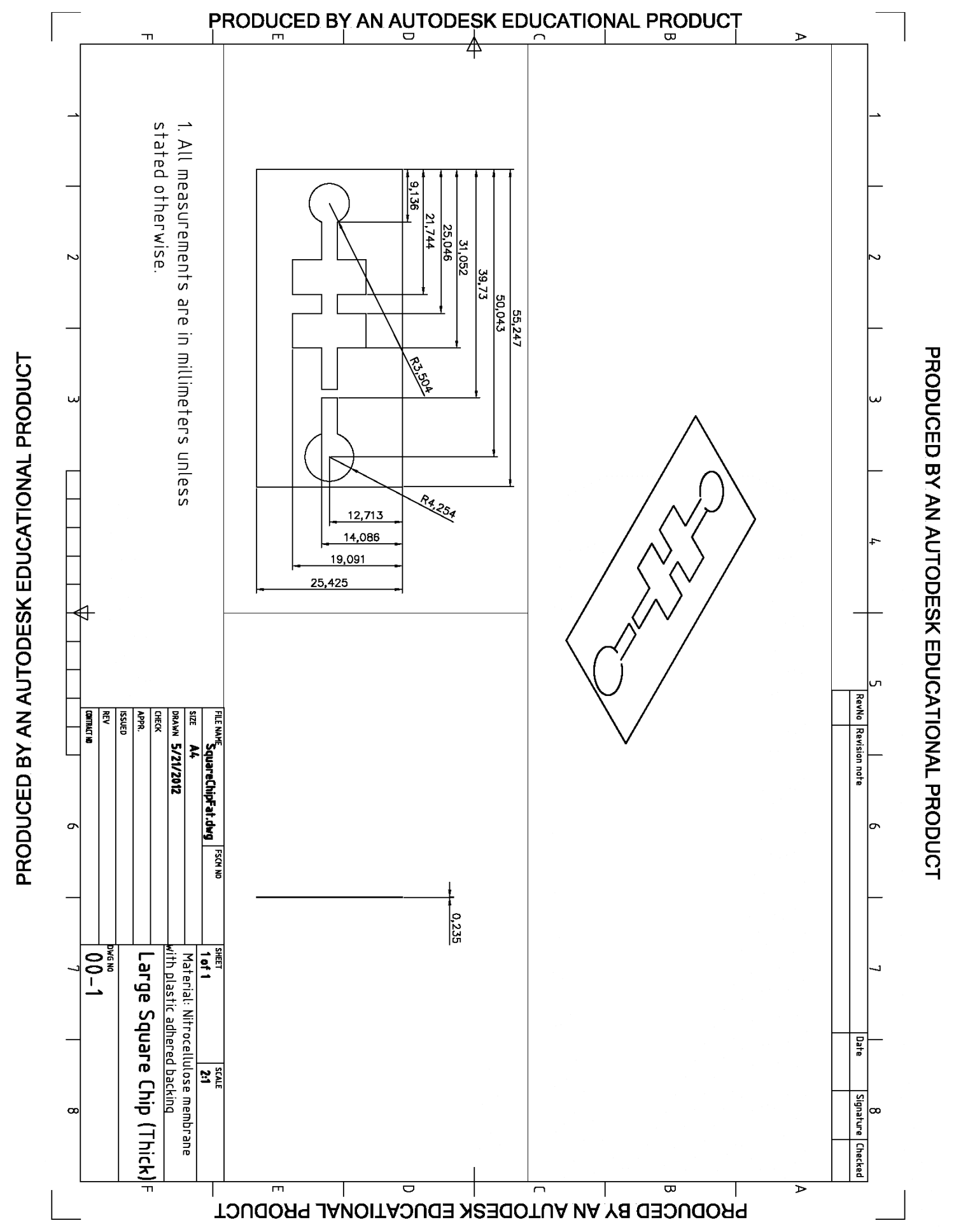




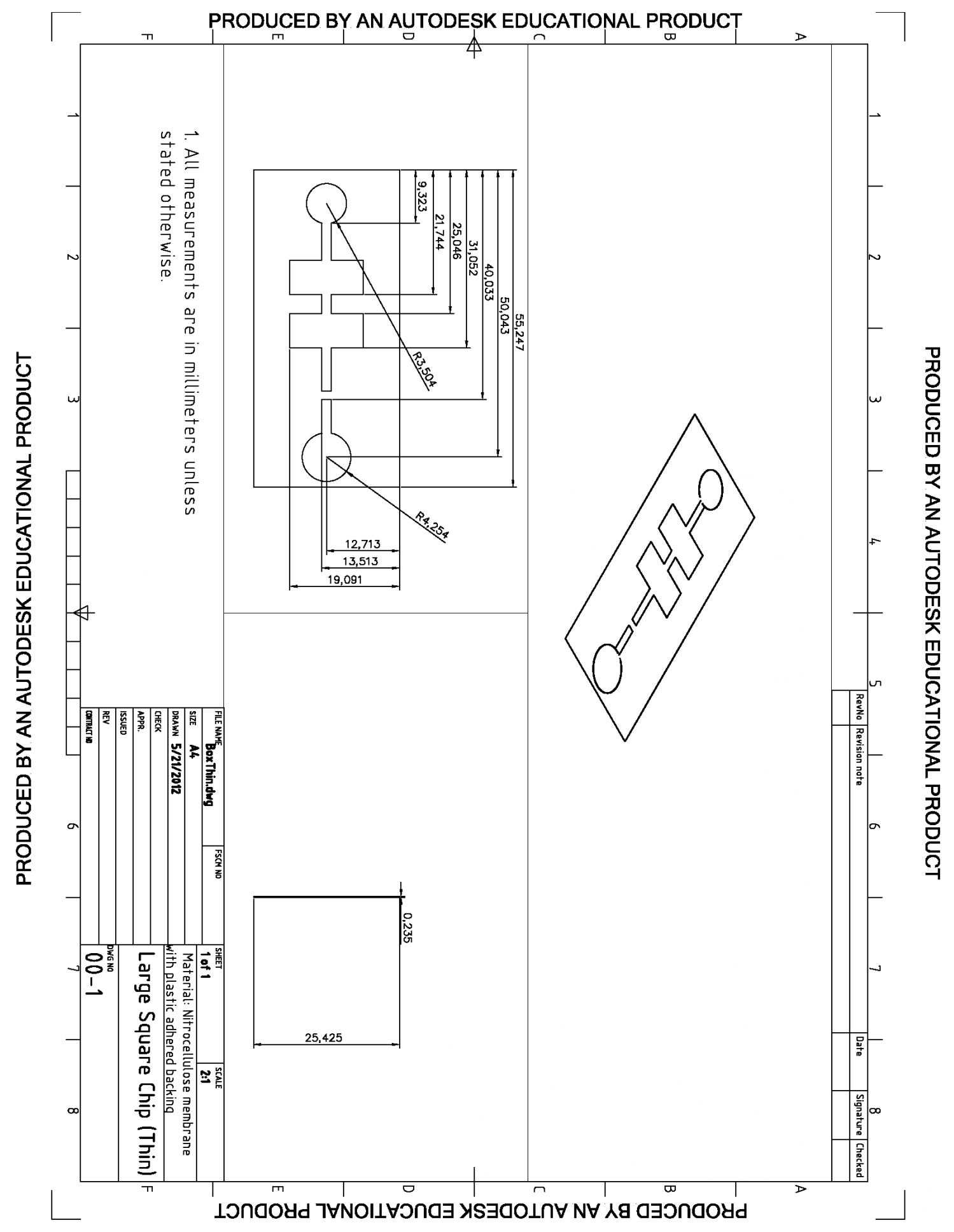




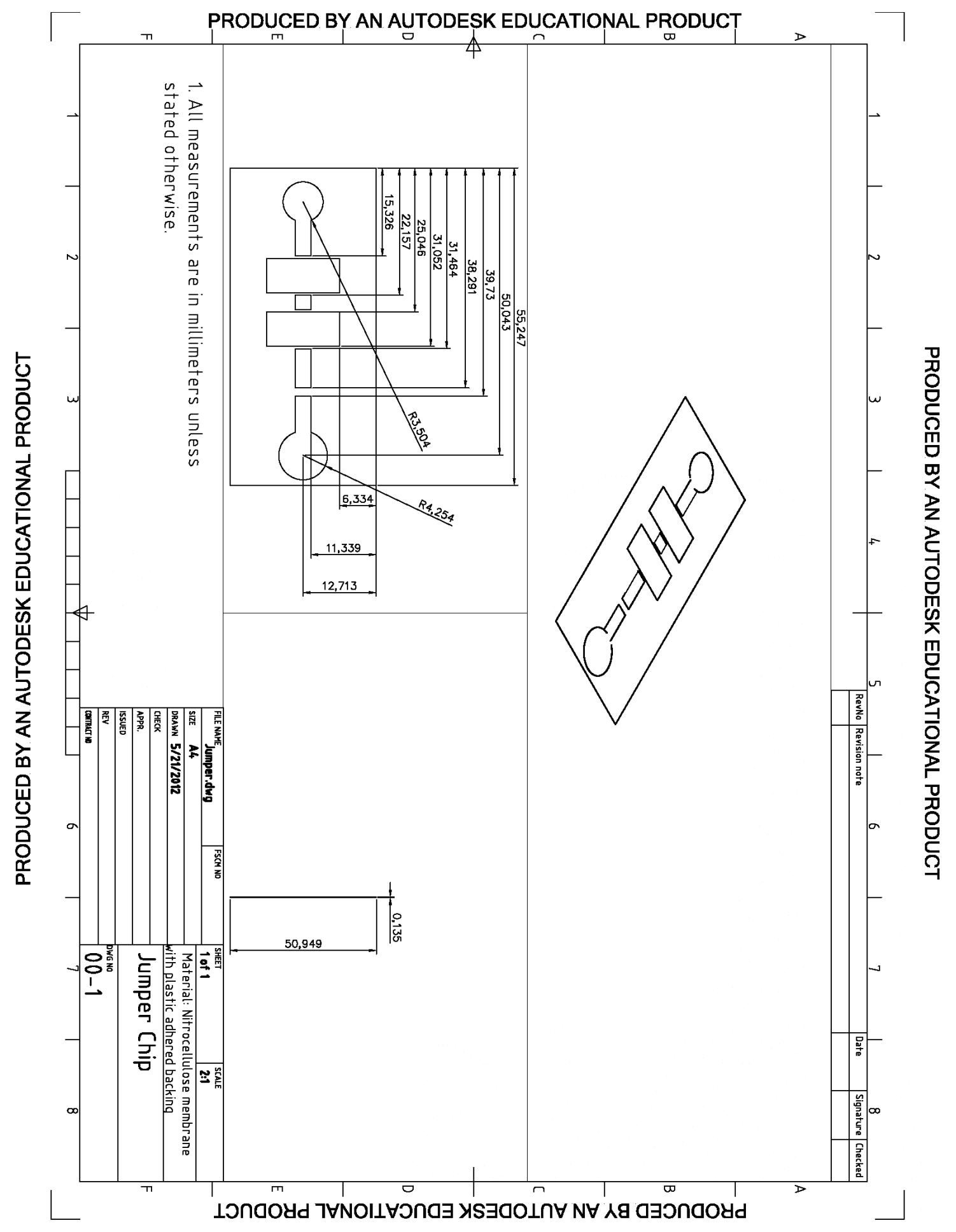




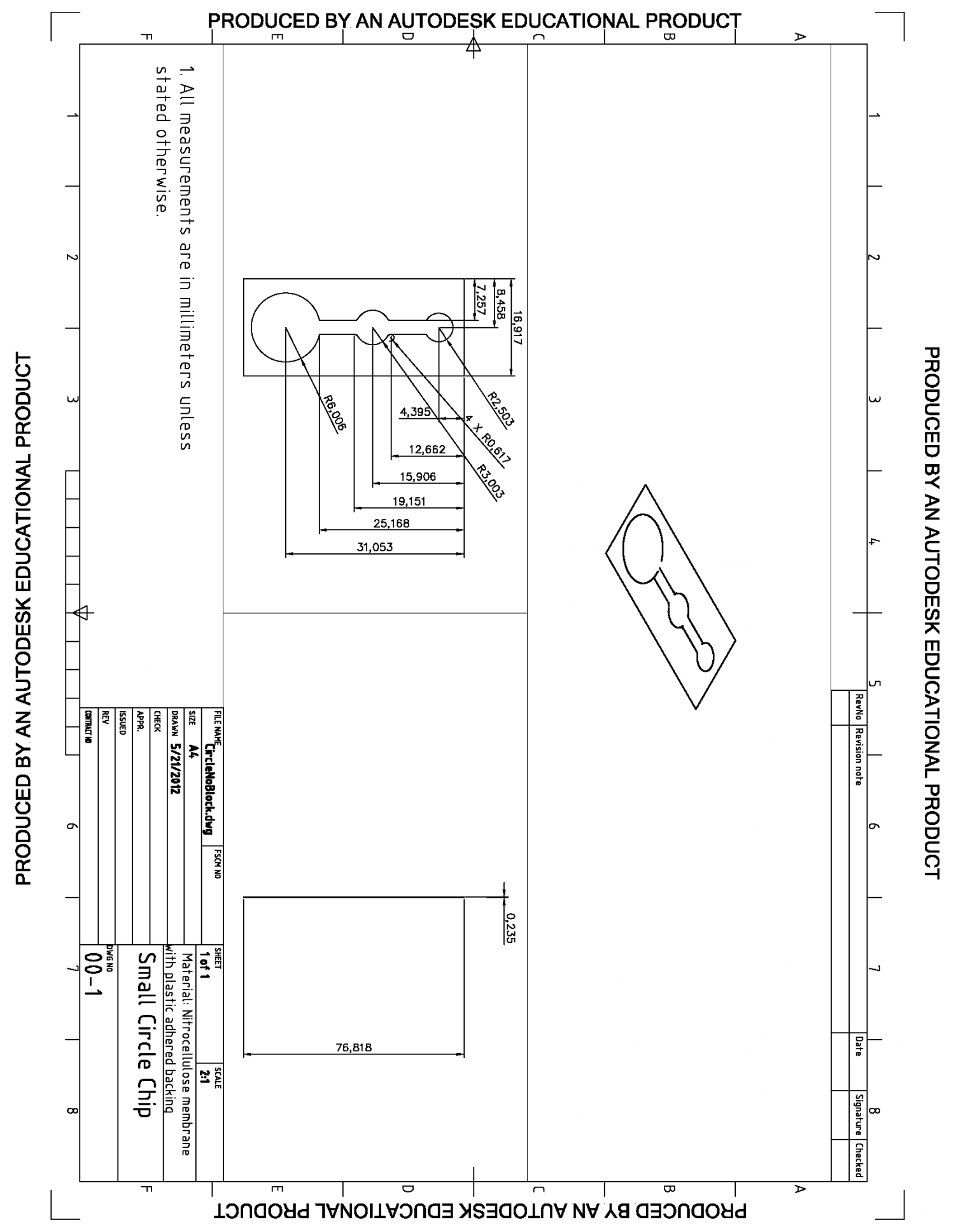




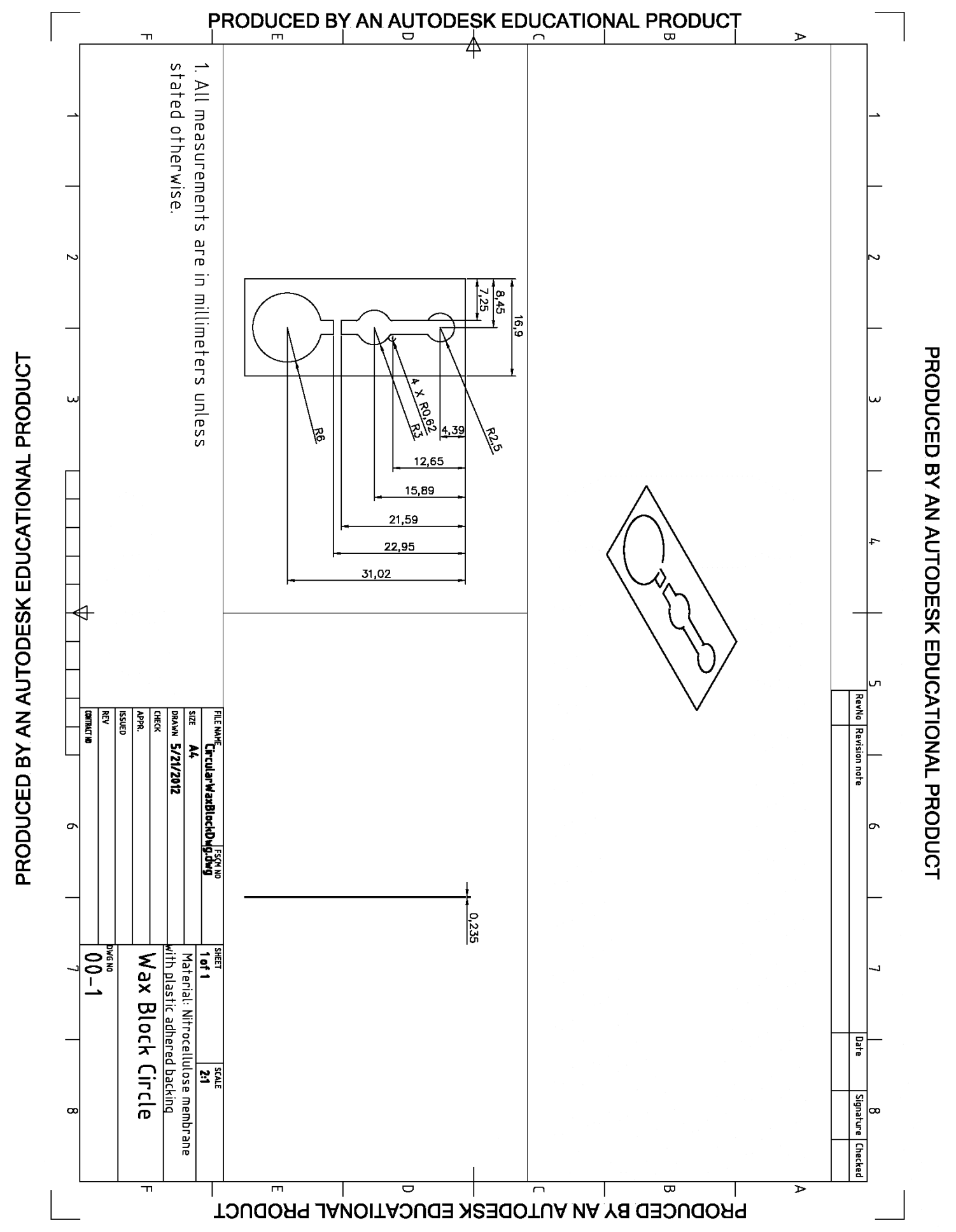




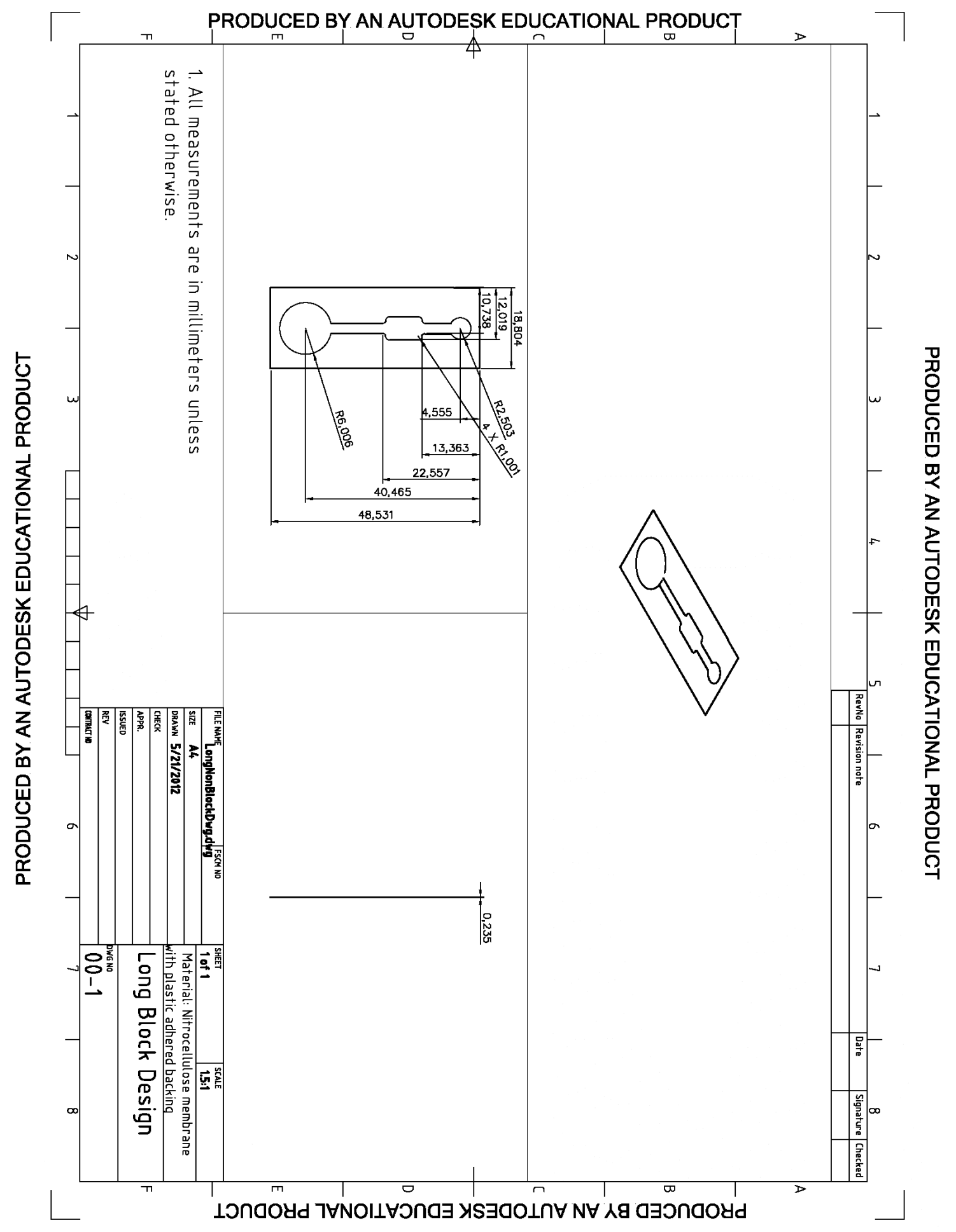




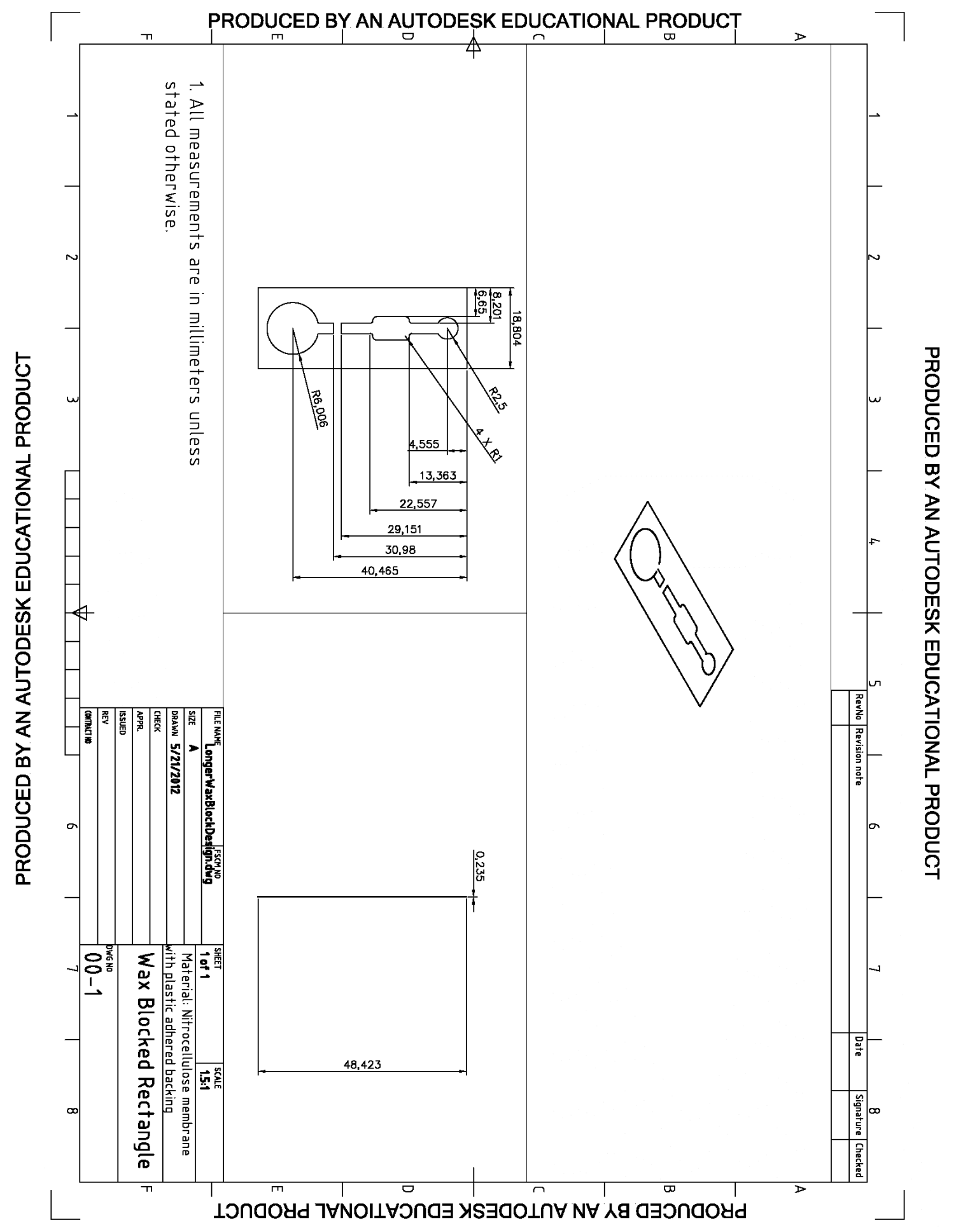




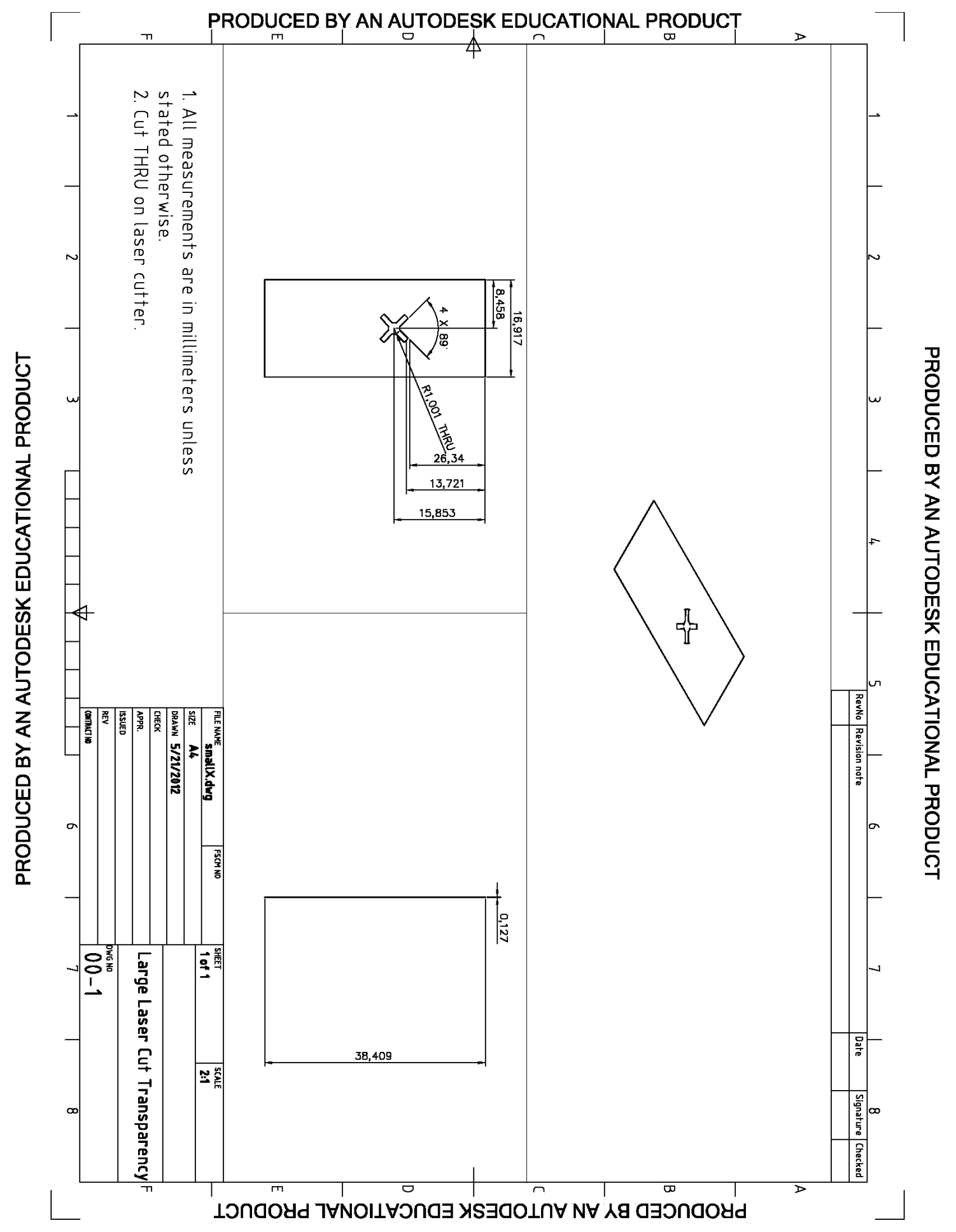




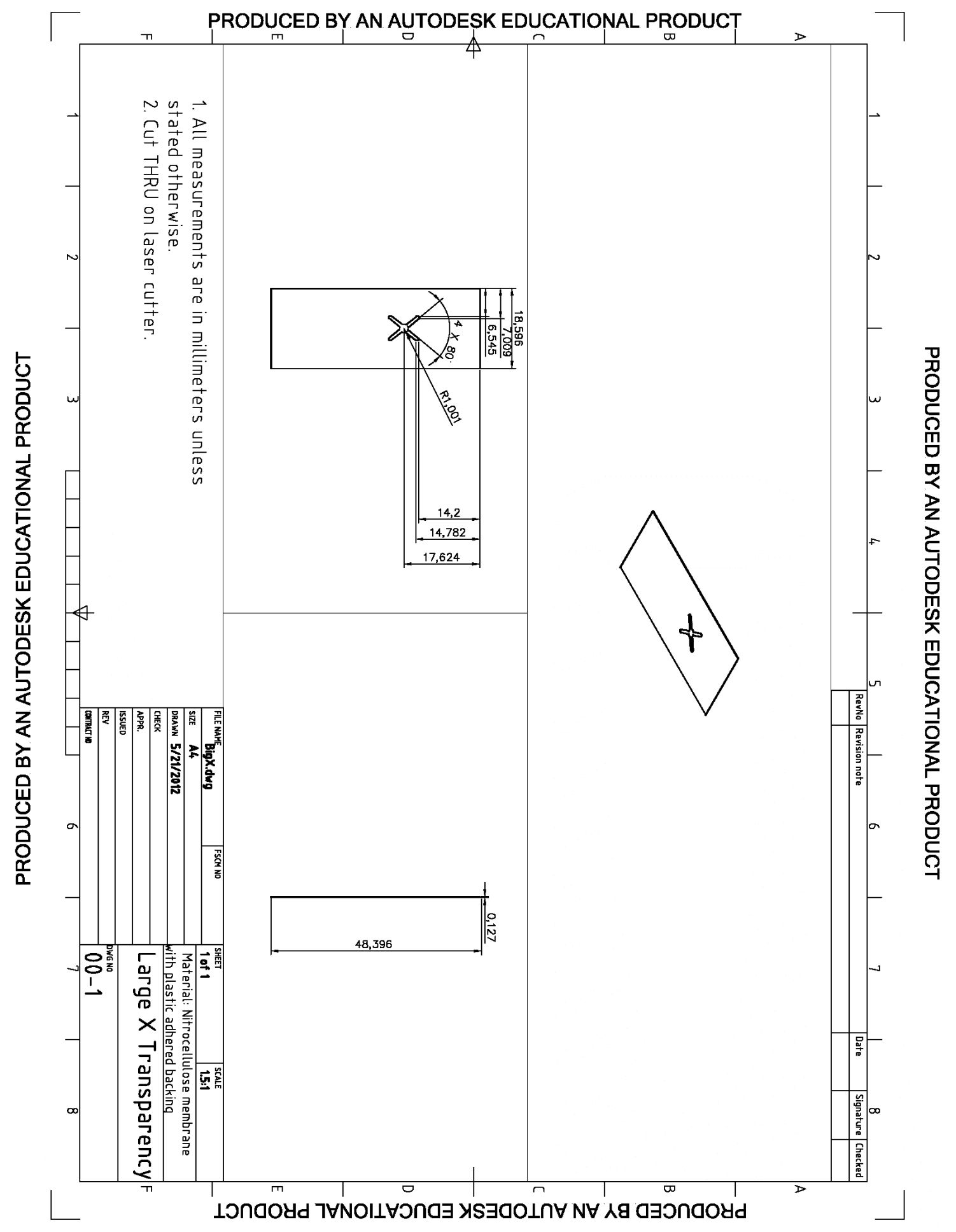




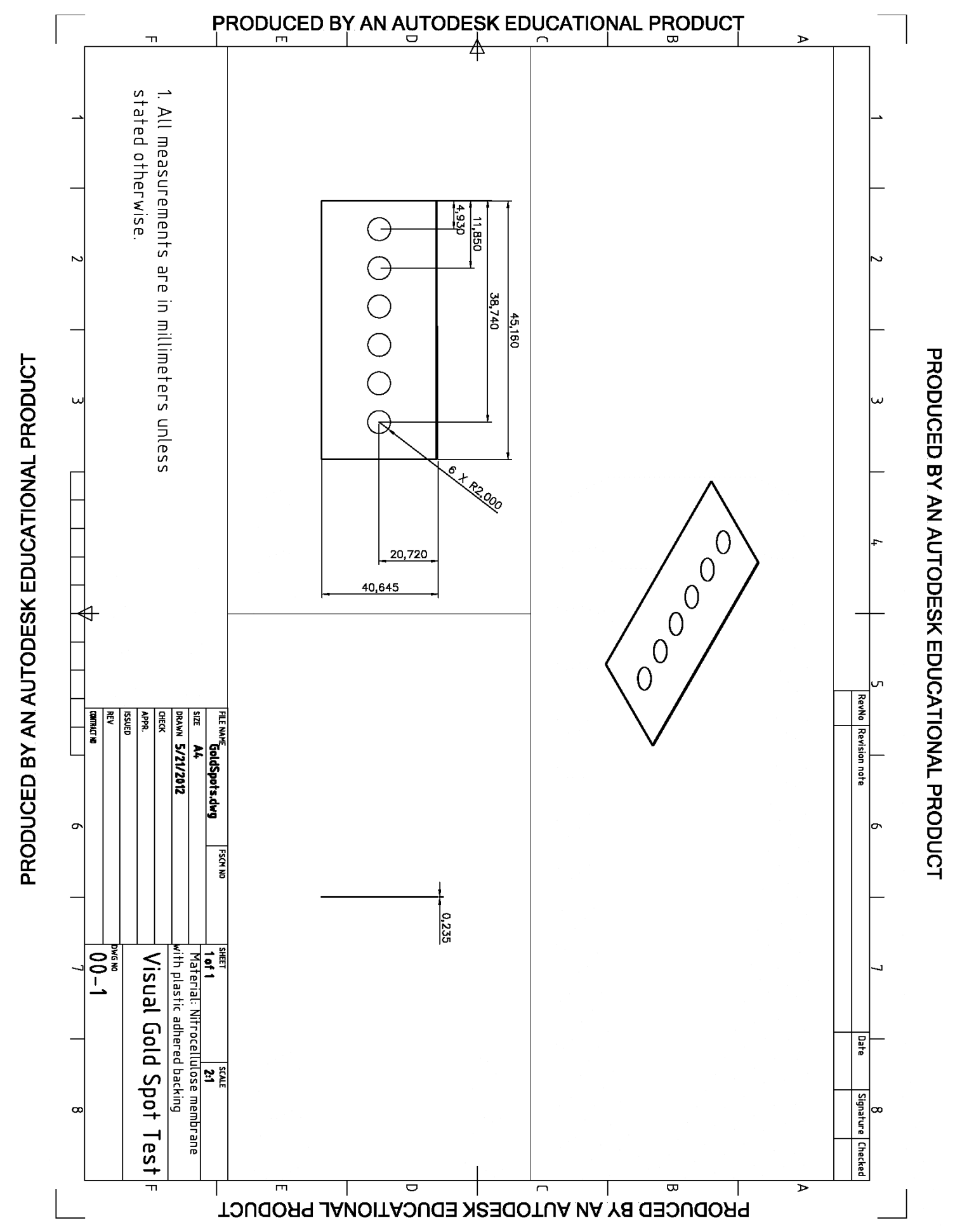




\section{Appendix D: Biochemistry Dilution Calculations}

\section{Diluting anti-VEGF Antibodies Conjugated With Gold}

- According to literature, the antibody should be diluted to anywhere between 1 $\mathrm{ng} / \mathrm{ml}$ to $2 \mu \mathrm{g} / \mathrm{ml}$

- However, this range of concentrations are too dilute to see any gold with the naked eye, therefore the solution was diluted to $0.5 \mathrm{mg} / \mathrm{ml}$ for the sake of visual detection

- Given $100 \mathrm{ug}$ of antibody and adding $0.2 \mathrm{ml}$ of $0.01 \mathrm{M}$ PBS (pH 7.4) stock solution, this provides $0.5 \mathrm{mg} / \mathrm{ml}$ of antibody

- Store this antibody at 2-4 degrees Celsius and it will be stable for two weeks of use

\section{Diluting Polyclonal anti-VEGF Antibodies}

- The polyclonal antibodies were provided as $0.5 \mathrm{mg} / \mathrm{ml}$ by the company (in PBS)

- For further dilution, add 1X PBS

- These must be stored at -20 degrees Celsius and fully thawed before every use

\section{Diluting VEGF}

- VEGF was diluted into concentrations of $1.5 \mathrm{mg} / \mathrm{ml}, 10 \mathrm{ug} / \mathrm{ml}$, and $50 \mathrm{ng} / \mathrm{ml}$ using $1 \% \mathrm{BSA}$ as recommended by the provider

- Instructions state that when the lyophilized VEGF is dissolved in $135 \mathrm{ul}$ of $1 \%$ BSA it provides a concentration of $1.5 \mathrm{mg} / \mathrm{ml}$ 
- For the $10 \mathrm{ug} / \mathrm{ml}$ concentration, $10 \mathrm{ul}$ was removed from the $1.5 \mathrm{mg} / \mathrm{ml}$ concentration and combined with $1.5 \mathrm{~mL}$ of BSA $\left(\right.$ concentration $\left._{1}\right)\left(\right.$ volume $\left._{1}\right)=\left(\right.$ concentration $\left._{2}\right)\left(\right.$ volume $\left._{2}\right)$ $(10 u l)(1.5 \mathrm{mg} / \mathrm{ml})=(50 \mathrm{ng} / \mathrm{ml})\left(\right.$ volume $\left._{2}\right)$ volume $_{2}=1.5 \mathrm{~mL}$

- For the $50 \mathrm{ng} / \mathrm{ml}$ concentration, $50 \mathrm{ul}$ of the VEGF solution was removed from the $10 \mathrm{ug} / \mathrm{ml}$ concentration and $10 \mathrm{~mL}$ of $1 \%$ BSA was added $\left(\right.$ concentration $\left._{1}\right)\left(\right.$ volume $\left._{1}\right)=\left(\right.$ concentration $\left._{2}\right)\left(\right.$ volume $\left._{2}\right)$ $(10 \mathrm{ug} / \mathrm{ml})\left(\right.$ volume $\left._{1}\right)=(10 \mathrm{~mL})(50 \mathrm{ng} / \mathrm{mL})$ volume $_{1}=50 u \mathrm{~L}$

- All VEGF should be placed at -20 degrees Celsius to be used in 1 year

\section{Diluting IgG Antibodies}

- $\mathrm{IgG}$ was provided in a liquid form at a concentration of $2.4 \mathrm{mg} / \mathrm{ml}$, with $2.0 \mathrm{mg}$ of total solution

- $0.01 \mathrm{M}$ sodium phosphate and $0.25 \mathrm{M} \mathrm{NaCl}$ were needed for diluting the $\mathrm{IgG}$

- According to literature, the antibody should be diluted to $1 \mathrm{mg} / \mathrm{ml}$

- Calculations for appropriate dilution:

$\frac{\text { mass }}{\text { concentration }}=$ volume

$$
\begin{aligned}
& 2.0 \mathrm{mg} \quad \text { in order to find the total volume of stock } \mathrm{IgG} \\
& \frac{2.0 m g}{2.4 m g / m l}=0.833 m l \\
& \left(\text { concentration }_{1}\right)\left(\text { volume }_{1}\right)=\left(\text { concentration }_{2}\right)\left(\text { volume }_{2}\right) \\
& (2.4 \mathrm{mg} / \mathrm{ml})(0.833 \mathrm{ml})=(1.0 \mathrm{mg} / \mathrm{ml})\left(\text { volume }_{2}\right)
\end{aligned}
$$


volume $_{2}=2 \mathrm{ml}$ where $2 \mathrm{ml}$ represents the buffer solution necessary to dilute the $\operatorname{IgG}$

- Calculations for $0.01 \mathrm{M}$ sodium phosphate $\left(\right.$ concentration $\left._{1}\right)\left(\right.$ volume $\left._{1}\right)=\left(\right.$ concentration $\left._{2}\right)\left(\right.$ volume $\left._{2}\right)$

The stock solution of sodium phosphate is $0.05 \mathrm{M}$, therefore:

$(0.5 \mathrm{~mol} / \mathrm{L})\left(\right.$ volume $\left._{1}\right)=(0.01 \mathrm{~mol} / L)(0.1 \mathrm{~L})$

$0.1 \mathrm{~L}$ was chosen to demonstrate an overall volume of water to lower the molarity of sodium phosphate from $0.5 \mathrm{M}$ to $0.01 \mathrm{M}$. This number can change based on how much stock solution is wanted.

volume $_{1}=0.002 \mathrm{~L}$ of sodium phosphate

volume $_{2}=100 \mathrm{~mL}$ of water

- Calculations for $0.25 \mathrm{M} \mathrm{NaCl}$

In this scenario, making $100 \mathrm{~mL}$ of $0.25 \mathrm{M}$ stock solution

$(0.25$ moles $/ L)(100 \mathrm{~mL})=0.025$ moles

Molecular weight of $\mathrm{NaCl}=22.99+35.453=58.443$ grams

weight $=(n)(M W)$

weight $=(0.024$ moles $)(58.443$ grams $)$

weight $=1.461075$ grams

- Therefore, weigh out 1.461 grams of $\mathrm{NaCl}$ and combine with $100 \mathrm{~mL}$ of water

- Add $2 \mathrm{~mL}$ of sodium phosphate to the $0.25 \mathrm{M} \mathrm{NaCl}$ solution

- $\quad$ Measure the $\mathrm{pH}$ to ensure it is around 7.6

- Store solution at 2-4 degrees Celsius 


\section{1\% BSA Solution}

- Measure out 5 grams of lyophilized BSA

- Add $500 \mathrm{~mL}$ of buffer solution (water) to reconstitute for $1 \%$ BSA

\section{1\% Sucrose Solution}

- Measure out 5 grams of sucrose

- Add $500 \mathrm{~mL}$ of buffer solution (in this case the borate) to reconstitute for $1 \%$ sucrose solution

\section{$5 \%$ Sucrose Solution}

- Measure out 5 grams of sucrose

- Add $100 \mathrm{~mL}$ of buffer solution (in this case water) to reconstitute for $5 \%$ sucrose solution

\subsection{5\% SDS Solution}

- Use $100 \%$ stock solution of SDS

- Weigh 0.05 grams of SDS in $100 \mathrm{~mL}$ of water for $0.05 \%$

\section{$5 \mathrm{mM} \mathrm{Na}_{2} \mathrm{HPO}_{4}$ with a pH of 7.5}

- The stock solution of Na2HPO4 was originally $0.5 \mathrm{M}$ and needed to be diluted further for nitrocellulose post treatment $\left(\right.$ concentration $\left._{1}\right)\left(\right.$ volume $\left._{1}\right)=\left(\right.$ concentration $\left._{2}\right)\left(\right.$ volume $\left._{2}\right)$

- $(0.5 \mathrm{M})\left(\right.$ volume $\left._{1}\right)=(5 \mathrm{mM})(5 \mathrm{~mL})$ volume $_{1}=50 \mu \mathrm{L}$ 
- Where volume1 is the amount of stock that should be removed and combined with $5 \mathrm{~mL}$ of water in order to make a $5 \mathrm{mM}$ solution

\section{2 mM Borate Solution}

- $\quad$ Borate Buffer $\mathrm{pH} 7.4-9.2$

- Borax(sodium tetraborate) $0.2 \mathrm{M}=76.2 \mathrm{gm} / \mathrm{ml}$

- $\quad \mathrm{Na}_{2} \mathrm{~B}_{4} 0_{7} * 120 \mathrm{H}_{2} \mathrm{O}(\mathrm{MW}=381.37)$

- $\quad$ Boric acid $0.2 \mathrm{M}=12.37 \mathrm{gm} / 1$

- $\mathrm{H}_{3} \mathrm{BO}_{3}(\mathrm{MW}=61.83)$

- Add boric acid to borax solution until desired $\mathrm{pH}$ is reached. Dilute to desired molarity with $\mathrm{ddH}_{2} \mathrm{O}$.

\section{Diluting Aptamers Without Biotinylated End}

- Briefly spin the tube containing the lyophilized powder VEGF oligonucleotide prior to opening

- $\quad$ Pour distilled water into a beaker and $\mathrm{pH}$ appropriately to allow a $\mathrm{pH}$ between 7.0 and 8.0

○ Do this by adding $\mathrm{pH}$ buffer to the solution, drop at a time, measuring, and testing by dipping a $\mathrm{pH}$ strip into the solvent

- Pipette $940.46 \mu \mathrm{L}$ of appropriate $\mathrm{pH}$ water

According to the given instructions, adding $47023 \mu \mathrm{L}$ of water will allow for 100 $\mu \mathrm{M}$ of aptamer solution. 


$$
\begin{aligned}
& \frac{c}{47.023 \mathrm{~mL}}=100 \mu \mathrm{M} \\
& c=4.7023 \times 10^{-6} \mathrm{~mol} \\
& \frac{4.7023 \times 10^{-6} \mathrm{~mol}}{x}=5 \mathrm{mM} \\
& x=\frac{4.7023 \times 10^{-6} \mathrm{~mol}}{5 \times 10^{-3} \mathrm{~mol} / \mathrm{L}} \\
& x=940.46 \mu \mathrm{L}
\end{aligned}
$$

This allows for a concentration of

$$
\frac{41185.7 u g}{940.46 u L}=43.79 u g / u l
$$

However, literature provides that aptamers should have a concentration of 1 $\mathrm{mg} / \mathrm{ml}$

$\left(\right.$ concentration $\left._{1}\right)\left(\right.$ volume $\left._{1}\right)=\left(\right.$ concentration $\left._{2}\right)\left(\right.$ volume $\left._{2}\right)$

$(43.79 \mu g / \mu L)($ volume 1$)=(1 \mathrm{mg} / \mathrm{ml})(1 \mathrm{ml})$

volume $1=22.8 \mu \mathrm{L}$

Therefore, remove $22.8 \mu \mathrm{L}$ of the diluted aptamer and combine with $1 \mathrm{~mL}$ of distilled water at appropriate $\mathrm{pH}$ for $1 \mathrm{mg} / \mathrm{ml}$ concentration

- Vortex well (at a speed of 10 for around one minute)

- Allow the solution to equilibrate for 5 minutes

- Vortex again to completely resuspend the aptamer

- Store the aptamer at -20 degrees Celsius (will maintain viability for up to 1 year)

\section{Diluting Aptamers With Biotinylated End}

- According to the given instructions, adding $45164 \mu \mathrm{L}$ of water will allow for 100 $\mu \mathrm{M}$ of aptamer solution. 


$$
\begin{aligned}
& \frac{c}{45.164 \mathrm{~mL}}=100 \mu \mathrm{m} \\
& c=4.5164 \times 10^{-6} \mathrm{~mol} \\
& \frac{4.5164 \times 10^{-6} \mathrm{~mol}}{x}=5 \mathrm{mM} \\
& x=\frac{4.5164 \times 10^{-6} \mathrm{~mol}}{5 \mathrm{mM}} \\
& x=\frac{4.5164 \times 10^{-6} \mathrm{~mol}}{5 \times 10^{-3} \mathrm{~mol} / \mathrm{L}} \\
& x=903.28 \mu \mathrm{L}
\end{aligned}
$$

The mass of the entire dried aptamer was $41389.2 \mu \mathrm{g}$. This allows for a concentration of:

$$
\frac{41389.2 \mu g}{903.28 \mu L}=45.82 \mu \mathrm{g} / \mu L
$$

However, literature provides that aptamers should have a concentration of 1 $\mathrm{mg} / \mathrm{ml}$.

$\left(\right.$ concentration $\left._{1}\right)\left(\right.$ volume $\left._{1}\right)=\left(\right.$ concentration $\left._{2}\right)\left(\right.$ volume $\left._{2}\right)$

$(45.82 \mu \mathrm{g} / \mu L)\left(\right.$ volume $\left._{1}\right)=(1 \mathrm{mg} / \mathrm{ml})(1 \mathrm{ml})$

volume $_{1}=21.82 \mu \mathrm{L}$

Therefore, remove $22.8 \mu \mathrm{L}$ of the diluted aptamer and combine with $1 \mathrm{~mL}$ of distilled water at appropriate $\mathrm{pH}$ for $1 \mathrm{mg} / \mathrm{ml}$ concentration.

Dilutions were calculated for six different concentrations of gold conjugated antibodies for a gold visual signal spot detection test. In Table XVI, the removed volume was how much solution was removed from the previous test concentration in order to make the next dilution. The volume of added buffer was how much was added to the removed gold to further dilute the material. Additionally, the total volume was how much was created 
from the removed volume added to the volume of added buffer. The volume after

removal was how much solution was left after the removed volume for that test occurred.

Finally, the total volume after one test incorporates how much material was left post-

removing the solution for the next test dilution, as well as how much was removed for the spot test.

\section{Table XVI: Gold Spot Test Values}

Volumes and concentrations for the gold spot test in order to determine what minimal concentration allowed for gold visualization.

\begin{tabular}{|c|c|c|c|c|c|c|}
\hline Test & $\mathbf{1}$ & $\mathbf{2}$ & $\mathbf{3}$ & $\mathbf{4}$ & $\mathbf{5}$ & $\mathbf{6}$ \\
\hline $\begin{array}{c}\text { Concentration } \\
\text { (mg/ml) }\end{array}$ & 1 & 0.5 & 0.1 & 0.05 & 0.01 & 0.002 \\
\hline $\begin{array}{c}\text { Spot Volume } \\
\text { (ml) }\end{array}$ & 0.0003 & 0.0003 & 0.0003 & 0.0003 & 0.0003 & 0.0003 \\
\hline $\begin{array}{c}\text { Removed } \\
\text { Volume (ml) }\end{array}$ & 0.0006 & 0.0006 & 0.0006 & 0.0006 & 0.0006 & 0.0006 \\
\hline $\begin{array}{c}\text { Volume } \\
\text { Added Buffer } \\
\text { (ml) }\end{array}$ & 0.1 & 0.0012 & 0.003 & 0.0012 & 0.003 & 0.003 \\
\hline $\begin{array}{c}\text { Total Volume } \\
\text { (ml) }\end{array}$ & 0.1 & 0.0018 & 0.0036 & 0.0018 & 0.0036 & 0.0036 \\
\hline $\begin{array}{c}\text { Total Volume } \\
\text { After } \\
\text { Removal (ml) }\end{array}$ & 0.1 & 0.0012 & 0.003 & 0.0012 & 0.003 & 0.003 \\
\hline $\begin{array}{c}\text { Total Volume } \\
\text { After 1 Test } \\
\text { (ml) }\end{array}$ & 0.0997 & 0.0009 & 0.0027 & 0.0009 & 0.0027 & 0.0027 \\
\hline Signal? & Yes & Yes & No & No & No & No \\
\hline
\end{tabular}




\section{Appendix E: Instructions on Running A BLAST Test}

Given an immunogen sequence (usually from a vendor) one can run a BLAST. This determines the specificity of an antibody, protein, or DNA sequence. In the case of this thesis, the immunogen sequence of the monoclonal antibody that was used was:

\section{PQTCKCSCKNT}

This letter sequence was through the 158-168 region of the entire 191 letter protein sequence.

Google "BLAST" and c lick the first link from the search result (Figure 43).

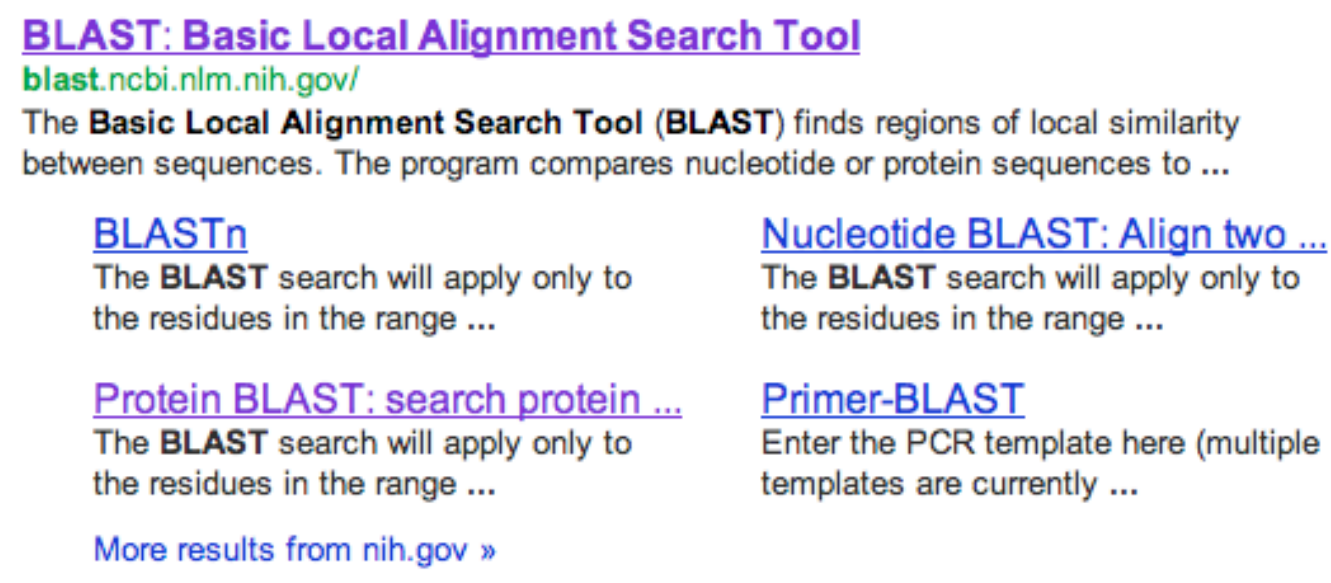

Figure 43: Google BLAST Link. Google result to link user to run a BLAST test for protein specificity.

For proteins and immunogens, click the "protein blast" link on the web page. Input the given immunogen/protein sequence into the highlighted yellow box and click the "BLAST" button at the bottom of the webpage (Figure 44). 


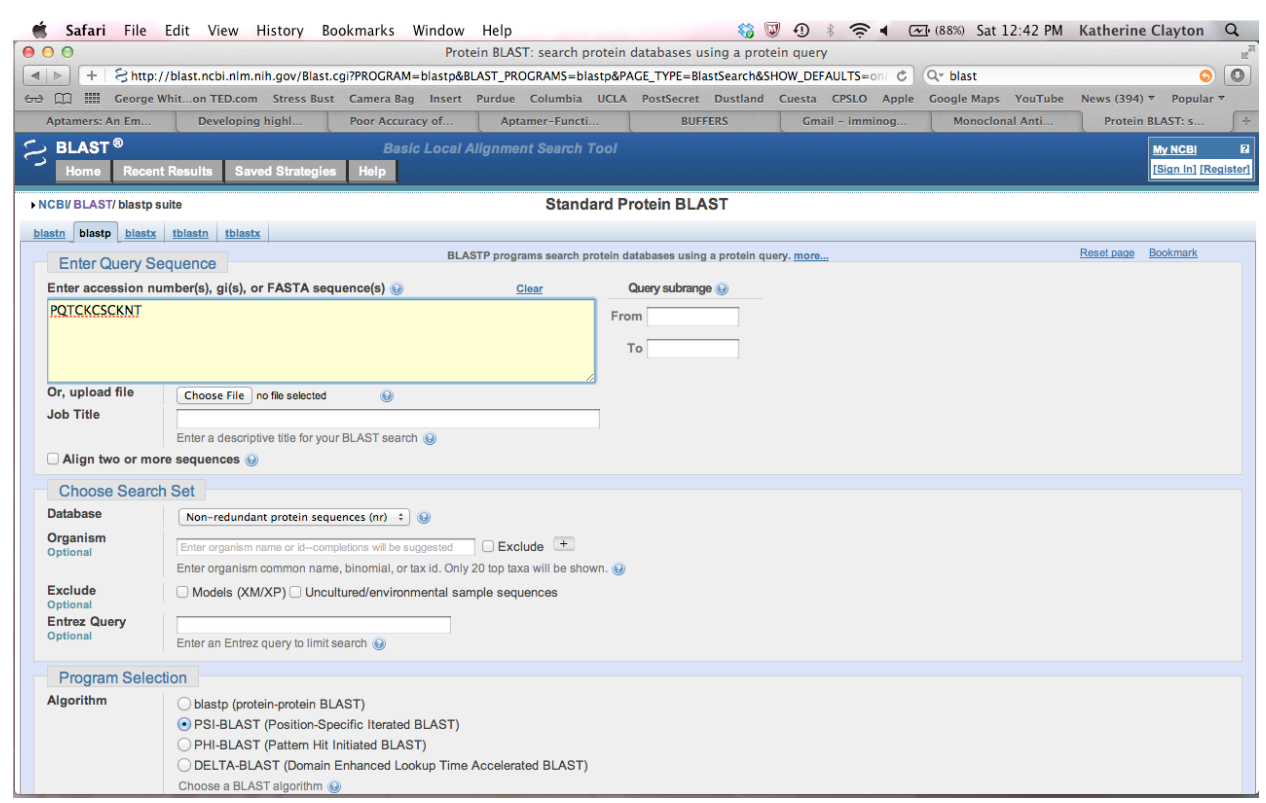

Figure 44: BLAST Testing. Inputting the sequence of the immunogen or protein in order to find its specificity.

Wait for 10-20 seconds, as database is being searched for a match to the sequence.

Then, the top of the page will have results that look similar to Figure 45.

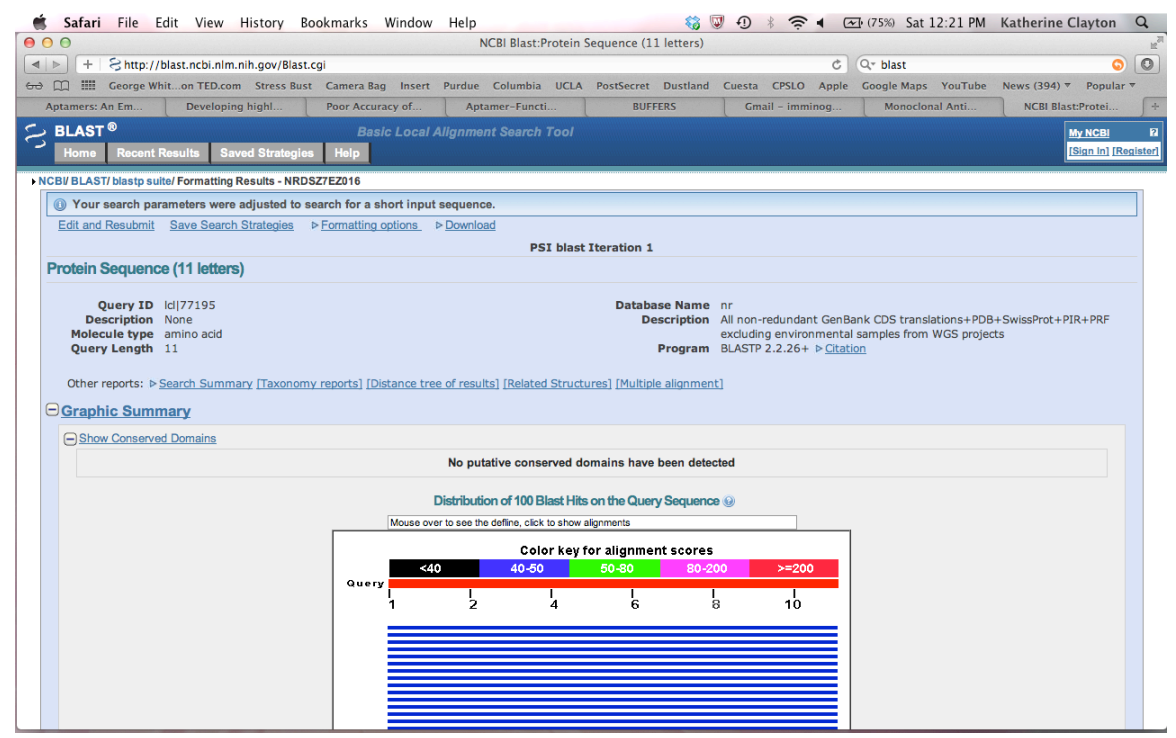

Figure 45: Results of BLAST. Resultant page from running the immunogen sequence for a monoclonal anti-VEGF antibody.

The results will yield proteins that are "above threshold". This means that there is a high significance (p-value of less than 0.05 ). That implies that these proteins are specific 
to the immunogen sequence that was searched. An example of this is demonstrated in Figure 46.

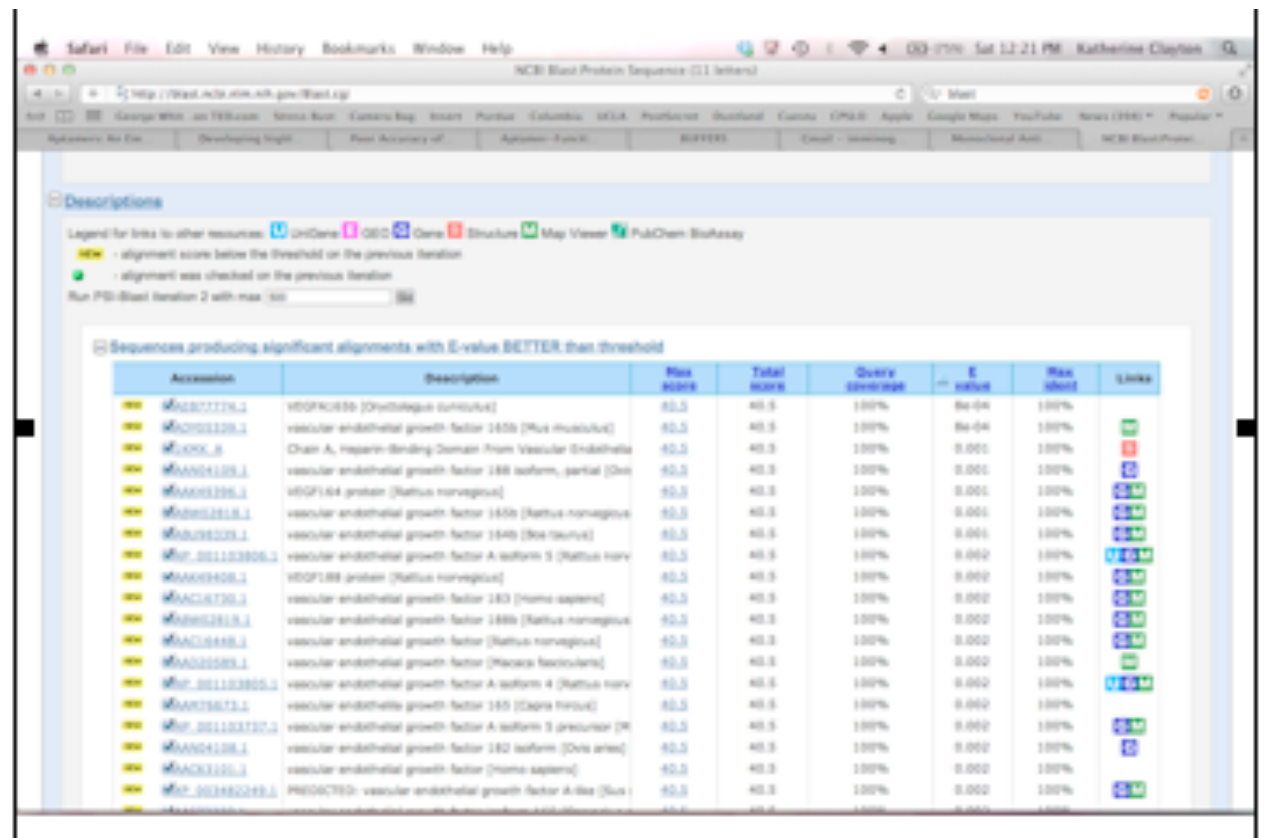

Figure 46: BLAST "Above Threshold". Demonstration of an "above threshold" list for proteins that show significance compared to the immunogen/protein sequence that is inputted.

On the bottom half of the search results there is a section called "worse than threshold." This means that the significance of the specificity of the protein/immunogen to those proteins has a significance of below 5\%. This implies that these are possible proteins with the binding sequence - but aren't very likely to be so. These results also link you to further scientific information about the proteins. BLAST is a unique tool in order to determine specificities when developing diagnostic tests. 


\section{Appendix F: Biochemistry Test Combinations}

Many antibody and aptamer tests were performed in order to see if visual signal would be provided after running the two-dimensional lateral flow assay test. The combinations tested (with no result) are presented in a series of tables. Table XVII and Table XVIII present the combinations of chip designs and application of gold to the chip for the monoclonal anti-VEGF antibody and IgG antibody, respectively. Table XIX and Table $X X$ present all data combination data for the experiments done involving the polyclonal anti-VEGF antibody. Finally, Table XXI comprises of data for experiments done with the pure aptamer (in other words, the aptamer with no biotinylated end).

Additionally, example images of the failed experiments are presented in this section. For instance, Figure 47 presents an example of spot test results from IgG whereas Figure 48 shows spot test results from VEGF. Likewise, Figure 49 presents "dead zones" or zones where the colloidal gold or other pigment sits throughout the lateral flow assay rather than wicking up and through the test. Additionally, test 8 done with the polyclonal antibody, where no colloidal gold is visible in either the detection region or the conjugate pad (as the pad has been washed out).

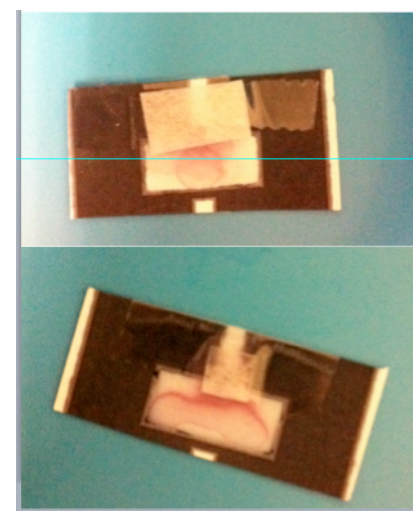

Figure 47: Spot testing for VEGF. Note how there is no distinct color or shape to prove detection of the VEGF. 
Table XVII: Monoclonal Anti-VEGF Antibody Design Combinations

\begin{tabular}{|c|c|c|c|c|}
\hline \multicolumn{5}{|c|}{ Monoclonal Anti-VEGF Antibody } \\
\hline Test & Gold Application & Input Test Type & $\begin{array}{c}\text { Drying } \\
\text { Technique }\end{array}$ & Design \\
\hline 1 & Conjugate Pad & $\begin{array}{l}\text { Lateral Flow } \\
\text { Assay }\end{array}$ & $\begin{array}{l}\text { Oven } 37 \\
\text { Degrees } 2 \\
\text { hours }\end{array}$ & $\begin{array}{l}\text { Large Square } \\
\text { Wells }\end{array}$ \\
\hline 2 & Conjugate Pad & $\begin{array}{l}\text { Lateral Flow } \\
\text { Assay }\end{array}$ & $\begin{array}{c}\text { Room } \\
\text { Temperature }\end{array}$ & $\begin{array}{l}\text { Large Square } \\
\text { Wells }\end{array}$ \\
\hline 3 & Conjugate Pad & $\begin{array}{l}\text { Lateral Flow } \\
\text { Assay }\end{array}$ & $\begin{array}{c}\text { Oven } 37 \\
\text { Degrees } 2 \\
\text { hours }\end{array}$ & $\begin{array}{l}\text { "Jumper" } \\
\text { Design }\end{array}$ \\
\hline 4 & Spotted & Spot Test & $\begin{array}{c}\text { Oven } 37 \\
\text { Degrees } 2 \\
\text { hours }\end{array}$ & $\begin{array}{c}\text { Single Square } \\
\text { Well }\end{array}$ \\
\hline 5 & Spotted & Spot Test & $\begin{array}{c}\text { Room } \\
\text { Temperature }\end{array}$ & $\begin{array}{c}\text { Single Square } \\
\text { Well }\end{array}$ \\
\hline 6 & Mixed Gold + VEGF & $\begin{array}{l}\text { Lateral Flow } \\
\text { Assay }\end{array}$ & $\begin{array}{c}\text { Oven } 37 \\
\text { Degrees } 2 \\
\text { hours }\end{array}$ & $\begin{array}{l}\text { Large Square } \\
\text { Wells }\end{array}$ \\
\hline 7 & Mixed Gold + VEGF & $\begin{array}{l}\text { Lateral Flow } \\
\text { Assay }\end{array}$ & $\begin{array}{c}\text { Room } \\
\text { Temperature }\end{array}$ & $\begin{array}{l}\text { Large Square } \\
\text { Wells }\end{array}$ \\
\hline 8 & Mixed Gold + VEGF & $\begin{array}{c}\text { Lateral Flow } \\
\text { Assay }\end{array}$ & $\begin{array}{c}\text { Oven } 37 \\
\text { Degrees } 2 \\
\text { hours }\end{array}$ & $\begin{array}{l}\text { "Jumper" } \\
\text { Design }\end{array}$ \\
\hline 9 & Mixed Gold + VEGF & $\begin{array}{l}\text { Lateral Flow } \\
\text { Assay }\end{array}$ & $\begin{array}{c}\text { Oven } 37 \\
\text { Degrees } 2 \\
\text { hours }\end{array}$ & $\begin{array}{l}\text { Small Square } \\
\text { Wells }\end{array}$ \\
\hline 10 & $\begin{array}{l}\text { Conjugate Pad + } \\
\text { Sucrose }\end{array}$ & $\begin{array}{l}\text { Lateral Flow } \\
\text { Assay }\end{array}$ & $\begin{array}{c}\text { Oven } 37 \\
\text { Degrees } 2 \\
\text { hours }\end{array}$ & $\begin{array}{l}\text { Large Square } \\
\text { Wells }\end{array}$ \\
\hline 11 & $\begin{array}{l}\text { Conjugate Pad + } \\
\text { Sucrose }\end{array}$ & $\begin{array}{l}\text { Lateral Flow } \\
\text { Assay }\end{array}$ & $\begin{array}{c}\text { Room } \\
\text { Temperature }\end{array}$ & $\begin{array}{l}\text { Large Square } \\
\text { Wells }\end{array}$ \\
\hline
\end{tabular}




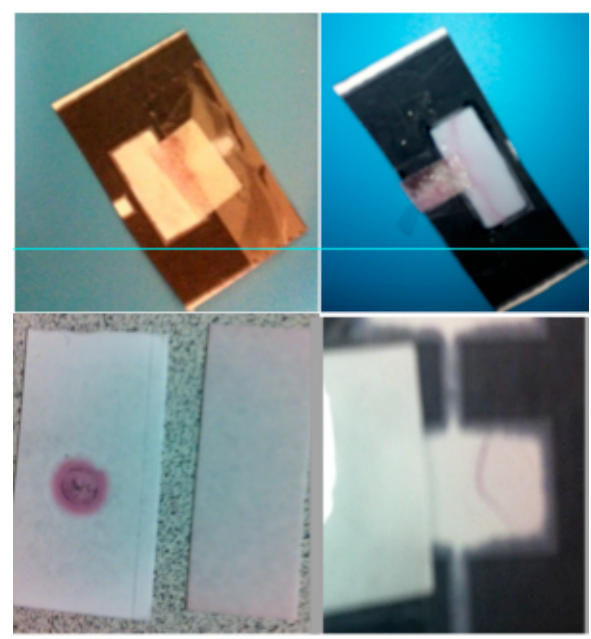

Figure 48: Spot testing for IgG. Note how there is no distinct color or shape to prove detection of the VEGF.

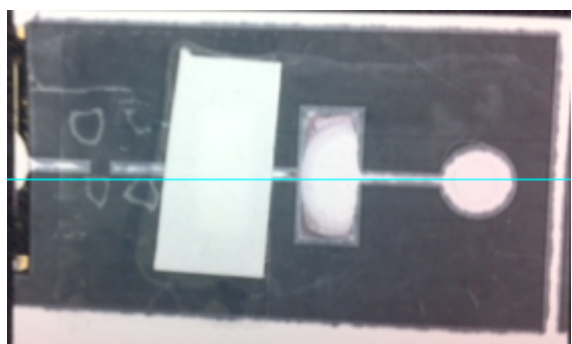

Figure 49: Design Dead Zones. The use of a more square shaped chip leads to "dead zones" - i.e. where the gold aggregates on the membrane.

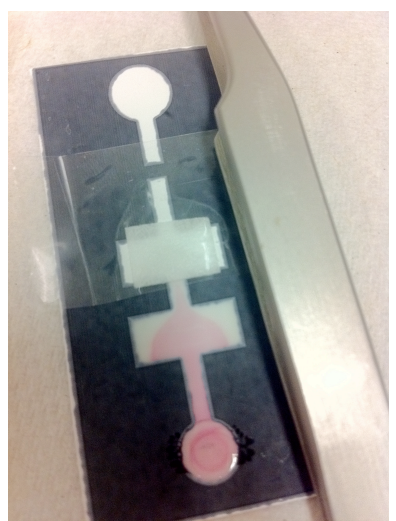

Figure 50: Chip Flow Pattern. Using the large square well design creates a unique flow pattern where in each well a parabolic shape is formed by the sample - this is important for comparing the unique shape of the gold in the detected aptamer chip (a spot shape) and the fact that this gold tends to eventually dilute out, whereas in the aptamer chip it did not. 
Table XVIII: IgG Antibody Design Combinations

\begin{tabular}{|c|c|c|c|c|}
\hline \multicolumn{5}{|c|}{ IgG Antibody } \\
\hline Test & Gold Application & Input Test Type & $\begin{array}{c}\text { Drying } \\
\text { Technique } \\
\end{array}$ & Design \\
\hline 1 & Conjugate Pad & $\begin{array}{c}\text { Lateral Flow } \\
\text { Assay }\end{array}$ & $\begin{array}{c}\text { Oven } 37 \\
\text { Degrees } 2 \\
\text { hours }\end{array}$ & $\begin{array}{l}\text { Large Square } \\
\text { Wells }\end{array}$ \\
\hline 2 & Conjugate Pad & $\begin{array}{l}\text { Lateral Flow } \\
\text { Assay }\end{array}$ & $\begin{array}{c}\text { Room } \\
\text { Temperature }\end{array}$ & $\begin{array}{l}\text { Large Square } \\
\text { Wells }\end{array}$ \\
\hline 3 & Conjugate Pad & $\begin{array}{c}\text { Lateral Flow } \\
\text { Assay }\end{array}$ & $\begin{array}{c}\text { Oven } 37 \\
\text { Degrees } 2 \\
\text { hours }\end{array}$ & $\begin{array}{l}\text { "Jumper" } \\
\text { Design }\end{array}$ \\
\hline 4 & Spotted & Spot Test & $\begin{array}{c}\text { Oven } 37 \\
\text { Degrees } 2 \\
\text { hours }\end{array}$ & $\begin{array}{c}\text { Single Square } \\
\text { Well }\end{array}$ \\
\hline 5 & Spotted & Spot Test & $\begin{array}{c}\text { Room } \\
\text { Temperature }\end{array}$ & $\begin{array}{l}\text { Single Square } \\
\text { Well }\end{array}$ \\
\hline 6 & Mixed Gold + VEGF & $\begin{array}{c}\text { Lateral Flow } \\
\text { Assay }\end{array}$ & $\begin{array}{c}\text { Oven } 37 \\
\text { Degrees } 2 \\
\text { hours }\end{array}$ & $\begin{array}{l}\text { "Jumper" } \\
\text { Design }\end{array}$ \\
\hline 7 & Mixed Gold + VEGF & $\begin{array}{l}\text { Lateral Flow } \\
\text { Assay }\end{array}$ & $\begin{array}{c}\text { Room } \\
\text { Temperature }\end{array}$ & $\begin{array}{l}\text { Large Square } \\
\text { Well }\end{array}$ \\
\hline
\end{tabular}

Table XIX: Polyclonal Anti-VEGF Post-Treatments

\begin{tabular}{|c|c|c|}
\hline \multicolumn{3}{|c|}{ Polyclonal Anti-VEGF Antibody } \\
\hline Test & Post-Treatment & Wash \\
\hline 1 & $1 \%$ BSA for 7 minutes & $0.05 \% \mathrm{SDS} / 5 \mathrm{mM} \mathrm{Na}_{2} \mathrm{HPO}_{4}$ for $10 \mathrm{~min}$ \\
\hline 2 & $1 \%$ BSA for 7 minutes & $0.05 \% \mathrm{SDS} / 5 \mathrm{mM} \mathrm{Na}_{2} \mathrm{HPO}_{4}$ for $10 \mathrm{~min}$ \\
\hline 3 & $1 \%$ BSA for 7 minutes & $0.05 \% \mathrm{SDS} / 5 \mathrm{mM} \mathrm{Na}_{2} \mathrm{HPO}_{4}$ for $10 \mathrm{~min}$ \\
\hline 4 & $1 \%$ BSA for 7 minutes & $0.05 \% \mathrm{SDS} / 5 \mathrm{mM} \mathrm{Na}_{2} \mathrm{HPO}_{4}$ for $10 \mathrm{~min}$ \\
\hline 5 & $1 \%$ BSA for 30 minutes & Water Wash - 1 time \\
\hline 6 & $1 \%$ BSA for 7 minutes & $0.05 \% \mathrm{SDS} / 5 \mathrm{mM} \mathrm{Na}_{2} \mathrm{HPO}_{4}$ for $10 \mathrm{~min}$ \\
\hline 7 & $1 \%$ BSA for 30 minutes & Water Wash - 1 time \\
\hline 8 & $1 \%$ BSA for 7 minutes & $0.05 \% \mathrm{SDS} / 5 \mathrm{mM} \mathrm{Na}_{2} \mathrm{HPO}_{4}$ for $10 \mathrm{~min}$ \\
\hline
\end{tabular}


Table XX: Polyclonal Anti-VEGF Antibody Design Combinations

\begin{tabular}{|c|c|c|c|c|}
\hline \multicolumn{5}{|c|}{ Polyclonal Anti-VEGF Antibody } \\
\hline Test & Gold Application & Input Test Type & $\begin{array}{c}\text { Drying } \\
\text { Technique }\end{array}$ & Design \\
\hline 1 & $\begin{array}{l}\text { Mixed Gold + } \\
\text { VEGF }\end{array}$ & $\begin{array}{c}\text { Lateral Flow } \\
\text { Assay }\end{array}$ & $\begin{array}{c}\text { Oven } 37 \text { Degrees } \\
2 \text { hours }\end{array}$ & $\begin{array}{l}\text { Small Circular } \\
\text { Well }\end{array}$ \\
\hline 2 & $\begin{array}{l}\text { Mixed Gold + } \\
\text { VEGF }\end{array}$ & $\begin{array}{c}\text { Lateral Flow } \\
\text { Assay }\end{array}$ & $\begin{array}{c}\text { Room } \\
\text { Temperature }\end{array}$ & $\begin{array}{c}\text { Small Circular } \\
\text { Well }\end{array}$ \\
\hline 3 & $\begin{array}{l}\text { Conjugate Pad - } \\
\text { Sucrose + Borate }\end{array}$ & $\begin{array}{c}\text { Lateral Flow } \\
\text { Assay }\end{array}$ & $\begin{array}{c}\text { Oven } 37 \text { Degrees } \\
2 \text { hours }\end{array}$ & $\begin{array}{c}\text { Small Circular } \\
\text { Well }\end{array}$ \\
\hline 4 & $\begin{array}{l}\text { Conjugate Pad - } \\
\text { Sucrose + Borate }\end{array}$ & $\begin{array}{c}\text { Lateral Flow } \\
\text { Assay }\end{array}$ & $\begin{array}{c}\text { Room } \\
\text { Temperature }\end{array}$ & $\begin{array}{c}\text { Small Circular } \\
\text { Well }\end{array}$ \\
\hline 5 & $\begin{array}{c}\text { Gold On Paper + } \\
\text { Sucrose }\end{array}$ & $\begin{array}{c}\text { Lateral Flow } \\
\text { Assay }\end{array}$ & $\begin{array}{c}\text { Room } \\
\text { Temperature }\end{array}$ & $\begin{array}{c}\text { Small Circular } \\
\text { Well }\end{array}$ \\
\hline 6 & $\begin{array}{c}\text { Gold On Paper + } \\
\text { Sucrose }\end{array}$ & $\begin{array}{c}\text { Lateral Flow } \\
\text { Assay }\end{array}$ & $\begin{array}{c}\text { Room } \\
\text { Temperature }\end{array}$ & $\begin{array}{c}\text { Small Circular } \\
\text { Well }\end{array}$ \\
\hline 7 & $\begin{array}{c}\text { Gold On Paper }+ \\
\text { Sucrose }\end{array}$ & $\begin{array}{c}\text { Lateral Flow } \\
\text { Assay }\end{array}$ & $\begin{array}{c}\text { Room } \\
\text { Temperature }\end{array}$ & $\begin{array}{c}\text { Rounded } \\
\text { Rectangular } \\
\text { Well }\end{array}$ \\
\hline 8 & $\begin{array}{l}\text { Conjugate Pad - } \\
\text { Sucrose + Borate }\end{array}$ & $\begin{array}{c}\text { Lateral Flow } \\
\text { Assay }\end{array}$ & $\begin{array}{c}\text { Room } \\
\text { Temperature + } \\
\text { Humidity }\end{array}$ & $\begin{array}{l}\text { Large Square } \\
\text { Well }\end{array}$ \\
\hline
\end{tabular}

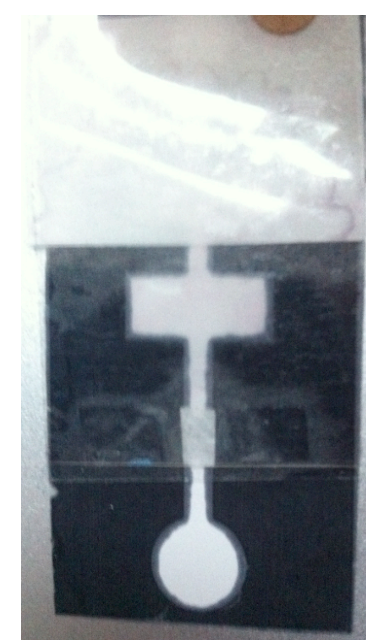

Figure 51: Results for the polyclonal VEGF antibody. Using the polyclonal anti-VEGF antibody as a capture reagent, it is seen that through the colloidal gold was eventually washed out of the conjugate pad, diluted, and absorbed, leaving no capturing or signal of VEGF in the detection region. 
Table XXI: Pure Aptamer Design Combinations

\begin{tabular}{|c|c|c|c|c|}
\hline \multicolumn{5}{|c|}{ Pure Aptamer } \\
\hline Test & Gold Application & $\begin{array}{l}\text { Input Test } \\
\text { Type }\end{array}$ & $\begin{array}{c}\text { Drying } \\
\text { Technique }\end{array}$ & Design \\
\hline 1 & $\begin{array}{c}\text { Conjugate Pad + } \\
\text { Sucrose }\end{array}$ & $\begin{array}{c}\text { Lateral Flow } \\
\text { Assay }\end{array}$ & $\begin{array}{c}\text { Room } \\
\text { Temperature }\end{array}$ & $\begin{array}{c}\text { Large Square } \\
\text { Wells }\end{array}$ \\
\hline 2 & $\begin{array}{c}\text { Conjugate Pad + } \\
\text { Sucrose }\end{array}$ & $\begin{array}{c}\text { Lateral Flow } \\
\text { Assay }\end{array}$ & $\begin{array}{c}\text { Oven } 37 \\
\text { Degrees } 2 \\
\text { hours }\end{array}$ & $\begin{array}{c}\text { Large Square } \\
\text { Wells }\end{array}$ \\
\hline 3 & Spotted & Spot Test & $\begin{array}{c}\text { Room } \\
\text { Temperature }\end{array}$ & $\begin{array}{c}\text { Single Square } \\
\text { Well }\end{array}$ \\
\hline 4 & Spotted & Spot Test & $\begin{array}{c}\text { Oven } 37 \\
\text { Degrees } 2 \\
\text { hours }\end{array}$ & $\begin{array}{c}\text { Single Square } \\
\text { Well }\end{array}$ \\
\hline 5 & Mixed Gold + VEGF & $\begin{array}{c}\text { Lateral Flow } \\
\text { Assay }\end{array}$ & $\begin{array}{c}\text { Room } \\
\text { Temperature }\end{array}$ & $\begin{array}{c}\text { Large Square } \\
\text { Well }\end{array}$ \\
\hline 6 & Mixed Gold + VEGF & $\begin{array}{c}\text { Lateral Flow } \\
\text { Assay }\end{array}$ & $\begin{array}{c}\text { Oven } 37 \\
\text { Degrees } 2 \\
\text { hours }\end{array}$ & $\begin{array}{c}\text { Large Square } \\
\text { Well }\end{array}$ \\
\hline
\end{tabular}




\section{Appendix G: Biochemistry Optimal Concentration Calculations}

\section{Chip Design Area/Volume Calculations}

In order to calculate the minimum volumes and concentrations of reagents to use for the two-dimensional lateral flow assay, the areas and volumes of the nitrocellulose chip were calculated. Initially the areas of parts of the chip (and the entirety of the chip) were calculated. The known identified values of radii and lengths are demonstrated in calculations, with their labels in Figure 52. Then, the chip was broken down to a first area and second area. This distinction may be found in Figure 53.

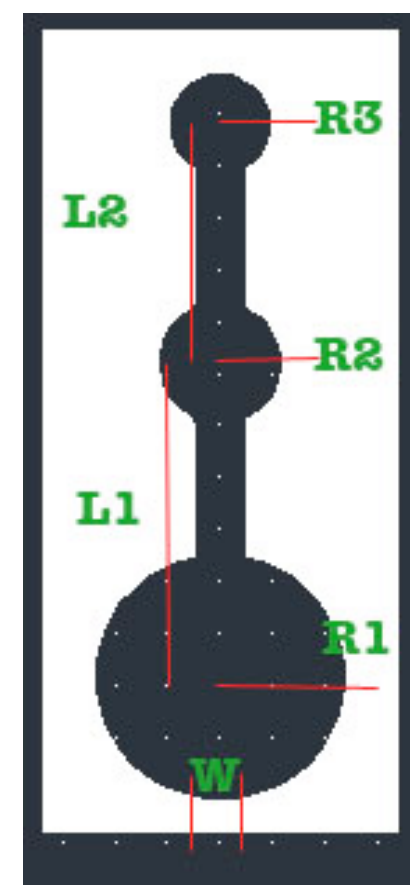

Figure 52: Chip Dimensions. Radii, width, and lengths of the two dimensional chip, labeled for the purposes of understanding the givens in the chip design area and volume calculations. 


\section{Total Chip Volume}

Givens:

$$
\begin{aligned}
& R_{1}=6 \mathrm{~mm} \\
& R_{2}=3 \mathrm{~mm} \\
& R_{3}=2.5 \mathrm{~mm} \\
& L_{1}=15.1315 \mathrm{~mm} \\
& L_{2}=11.5 \mathrm{~mm} \\
& W=2.40 \mathrm{~mm}
\end{aligned}
$$

\section{Calculation:}

$$
\begin{aligned}
& \text { Area }_{1}=\Pi\left(R_{1}\right)^{2} \\
& \text { Area }_{1}=\Pi(6 \mathrm{~mm})^{2} \\
& \text { Area }_{1}=36 \Pi \mathrm{mm}^{2} \\
& \text { Area }_{2}=\Pi\left(R_{2}\right)^{2} \\
& \text { Area }_{2}=\Pi(3 \mathrm{~mm})^{2} \\
& \text { Area }_{2}=9 \Pi \mathrm{mm}^{2} \\
& \text { Area }_{3}=\Pi\left(R_{3}\right)^{2} \\
& \text { Area }_{3}=\Pi\left(2.5 \mathrm{~mm}^{2}\right. \\
& \text { Area }_{3}=6.25 \Pi \mathrm{mm}^{2} \\
& \text { Area }_{4}=\left[L_{1}-R_{1}-R_{2}\right][\mathrm{W}] \\
& \text { Area }_{4}=\left[15.1315 \mathrm{~mm}^{2} 6 \mathrm{~mm}^{2}-3 \mathrm{~mm}\right][2.4 \mathrm{~mm}] \\
& \text { Area }_{4}=14.7156 \mathrm{~mm}^{2} \\
& \text { Area }_{5}=\left[L_{2}-R_{2}-R_{3}\right][\mathrm{W}] \\
& \text { Area }_{5}=\left[11.5 \mathrm{~mm}_{\text {total }}=3 \mathrm{~mm}-2.5 \mathrm{~mm}\right][2.4 \mathrm{~mm}] \\
& \text { Area }_{5}=14.4 \mathrm{~mm}^{2} \\
& \text { total }_{1}=190.12 \mathrm{~mm}^{2} \\
& \text { Ar }_{1}+A_{2}+A_{3}+A_{4}+A_{5} \\
&
\end{aligned}
$$




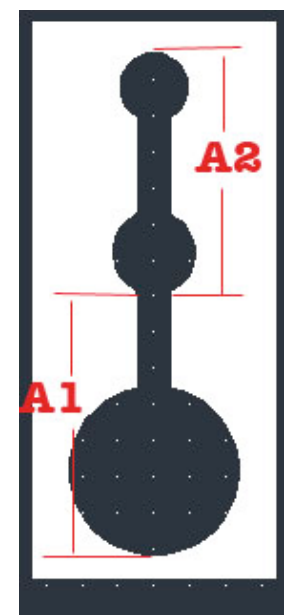

Figure 53: Chip Areas. Two-dimensional chip broken up into two distinct areas.

Area 1

$$
\begin{aligned}
& A_{1}=36 \Pi \mathrm{mm}^{2}+14.7156 \mathrm{~mm}^{2} \\
& A_{1}=127.813 \mathrm{~mm}^{2} \\
& V_{1}=A_{1} \times t \times \varepsilon \\
& V_{1}=\left(127.813 \mathrm{~mm}^{2}\right)(0.135 \mathrm{~mm})(0.82) \\
& V_{1}=14.15 \mathrm{~mm}^{3}
\end{aligned}
$$

Area 2

$$
\begin{aligned}
& A_{2}=9 \Pi \mathrm{mm}^{2}+6.25 \Pi \mathrm{mm}^{2}+14.4 \mathrm{~mm}^{2} \\
& A_{2}=62.309 \mathrm{~mm}^{2} \\
& V_{2}=A_{2} \times t \times \varepsilon \\
& V_{2}=\left(62.309 \mathrm{~mm}^{2}\right)(0.135 \mathrm{~mm})(0.82) \\
& V_{2}=6.898 \mathrm{~mm}^{3}
\end{aligned}
$$

Volume Fraction 
$V_{1} / V_{T}=\frac{14.15 \mathrm{~mm}^{3}}{14.15 \mathrm{~mm}^{3}+6.898 \mathrm{~mm}^{3}}$

$V_{1} / V_{T}=0.6723$

$V_{2} / V_{T}=\frac{6.898 \mathrm{~mm}^{3}}{14.15 \mathrm{~mm}^{3}+6.898 \mathrm{~mm}^{3}}$

$V_{2} / V_{T}=0.3277$

\section{$\underline{\mathrm{X}-\text { Spot Area/Volume Calculation }}$}

The area of the $\mathrm{X}$ region of the laser cut transparency was calculated, as well as its corresponding volume if applied to nitrocellulose membrane. This was in order to eventually calculate the minimum amount of antibody that could be applied to the detection region of the two-dimensional chip. This $\mathrm{X}$ is comprised of a circular center with four spokes. The labeled drawing may be found in Figure 54 .

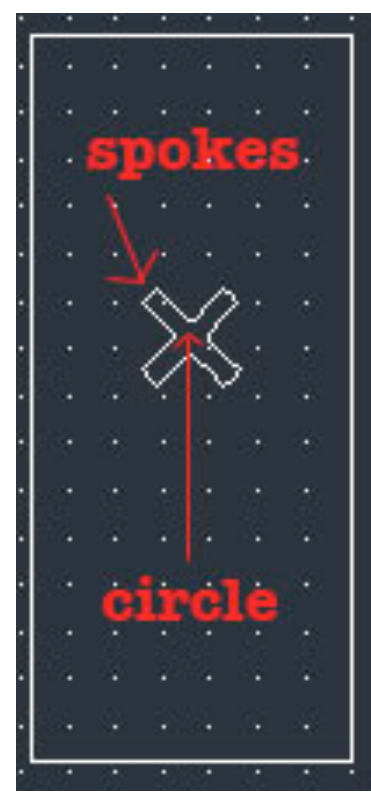

Figure 54: Described X-Shaped Design. AutoCAD representation of $X$ shaped design to strip detection antibody onto nitrocellulose membrane. The "spokes" and "circle" labels are for further understanding the calculations regarding antibody volume at the detection region. 
Area

Givens :

$R=1 \mathrm{~mm}$

$L=3 m m$

$W=0.8994 \mathrm{~mm}$

Calculation:

$A_{\text {spokes }}=(4)(3 \mathrm{~mm}-1 \mathrm{~mm})(0.8994 \mathrm{~mm})$

$A_{\text {spokes }}=7.1952 \mathrm{~mm}^{2}$

$A_{\text {circle }}=\Pi R^{2}$

$A_{\text {circle }}=\Pi(1 \mathrm{~mm})^{2}$

$A_{\text {circle }}=\Pi \mathrm{mm}^{2}$

$A_{\text {total }}=A_{\text {circle }}+A_{\text {spokes }}$

$A_{\text {total }}=\Pi \mathrm{mm}^{2}+7.1952 \mathrm{~mm}^{2}$

$A_{\text {total }}=10.34 \mathrm{~mm}^{2}$

Volume

$V_{X}=A_{X} \times t \times \varepsilon$

$V_{X}=\left(10.34 \mathrm{~mm}^{2}\right)(0.135 \mathrm{~mm})(0.82)$

$V_{X}=1.1445 \mathrm{~mm}^{3}$

Calculations for Detection Region and Gold-Antibody Conjugate Minimums

Givens:

- Molar mass of gold - 197 g/mole

- Mass concentration of gold conjugated antibody $-0.75 \mathrm{mg} / \mathrm{ml}$ (from visual gold experiments test) 
- Radius of gold colloid $-30 \mathrm{~nm}$ or $3.00 \mathrm{E}-8 \mathrm{~m}$

- Density of gold - $19.3 \mathrm{~g} / \mathrm{cc}\left(19300000 \mathrm{~g} / \mathrm{m}^{3}\right)$

\section{Volume per colloid:}

Assuming spherical shape of the colloidal particle

$$
\begin{aligned}
& \text { volume }=\left(\frac{4}{3}\right)(\Pi)(\text { radiuscolloid }) \\
& \text { volume }=\left(\frac{4}{3}\right)(\Pi)(30 \mathrm{~nm}) \\
& \text { volume }=1.13 \times 10^{-22} \mathrm{~m}^{3}
\end{aligned}
$$

\section{Mass per colloid:}

$$
\begin{aligned}
& \text { mass }_{\text {colloid }}=\text { volume }_{\text {colloid }} \times \text { density }_{\text {gold }} \\
& \text { mass }_{\text {colloid }}=\left(1.13 \times 10^{-22} \mathrm{~m}^{3}\right)\left(19300000 \mathrm{~g} / \mathrm{m}^{3}\right) \\
& \text { mass }_{\text {colloid }}=2.18 \times 10^{-15} \mathrm{~g} / \text { colloid }
\end{aligned}
$$

The experiment that analyzed the optimal mass concentration of colloidal gold by doing a spot test was used in order to calculate the volume of polyclonal antibody to spot down onto the nitrocellulose membrane (using the $\mathrm{X}$ pattern). $0.3 \mu \mathrm{L}$ of gold antibody was spotted down in a $2 \mathrm{~mm}$ diameter circle designed on nitrocellulose.

Grams of colloid in a $0.3 \mu$ L sample:

$$
\begin{aligned}
& \text { gramscolloid }_{\text {spottest }}=\text { concentration }_{\text {spottest }} \times \text { volume }_{\text {spotted }} \\
& \text { gramscolloid }_{\text {spottest }}=(0.75 \mathrm{mg} / \mathrm{ml})(0.3 \mu \mathrm{l}) \\
& \text { gramscolloid }_{\text {spottest }}=2.25 \times 10^{-7} \text { grams }^{\text {gram }}
\end{aligned}
$$

Minimum number of gold colloids in a $0.3 \mu L$ sample: 
\# colloids $=\frac{\text { gramscolloid }_{\text {spottest }}}{\text { mass }_{\text {colloid }}}$

\# colloids $=\frac{2.25 \times 10^{-7} \text { grams }}{2.18 \times 10^{-15} \text { grams } / \text { colloid }}$

\# colloids $=1.03 \times 10^{8}$ colloids

Adjusted Spot Volume:

This takes into consideration that the visual depth of the nitrocellulose paper was $10 \mu \mathrm{m}$ deep and that there is a porosity value of $82 \%$ for the membrane.

AdjustedVolume $_{\text {spottest }}=\Pi \times\left(\right.$ radius $^{2}($ porosity $)\left(\right.$ depth $\left._{\text {visual }}\right)$

AdjustedVolume $_{\text {spottest }}=\Pi \times(1 \mathrm{~mm})^{2}(0.82)(10 \mu \mathrm{m})$

AdjustedVolume $e_{\text {spottest }}=2.58 \times 10^{-11} \mathrm{~m}^{3}$

Adjusted X Volume:

AdjustedVolume $_{x}=\left(\right.$ area $\left._{x}\right)($ porosity $)\left(\right.$ depth $\left._{\text {visual }}\right)$

Adjusted Volume $=\left(10.34 \mathrm{~mm}^{2}\right)(0.82)(10 \mu \mathrm{m})$

AdjustedVolume $=8.48 \times 10^{-11} \mathrm{~m}^{3}$

Polyclonal Antibody Volume for X Region:

This was determined by doing a ratio for the spot volume with its adjusted membrane visible volume to finding the polyclonal antibody volume compared to the adjusted $\mathrm{X}$ area volume. 
$\frac{\text { SpotVolume }_{\text {reagent }}}{\text { AdjustedVolume }_{\text {spottest }}}=\frac{\text { XVolume }_{\text {reagent }}}{\text { AdjustedVolume }}$

XVolume $_{\text {reagent }}=\left(\frac{\text { SpotVolume }_{\text {reagent }}}{\text { AdjustedVolume }_{\text {spottest }}}\right)\left(\right.$ AdjustedVolume $\left._{x}\right)$

$X_{\text {Volume }}$ reagent $=\left(\frac{0.3 \mu L}{2.58 \times 10^{-11} \mathrm{~m}^{3}}\right)\left(8.48 \times 10^{-11} \mathrm{~m}^{3}\right)$

XVolume $_{\text {reagent }}=0.986 \mu \mathrm{L}$

Grams of colloid in a $0.986 \mu L$ sample:

gramscolloid $_{x}=$ concentration $_{\text {spottest }} \times$ volume $_{x}$

gramscolloid $_{x}=(0.75 \mathrm{mg} / \mathrm{ml})(0.986 \mu \mathrm{L})$

gramscolloid $_{x}=7.407 \times 10^{-7}$ grams

Minimum number of gold colloids in a $4.94 \mu L$ sample:

\# colloids $_{x}=\frac{\text { gramscolloid }_{x}}{\text { mass }_{\text {colloid }}}$

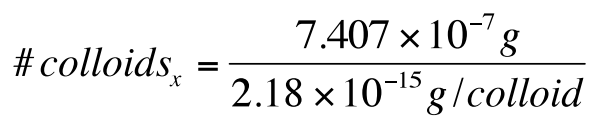

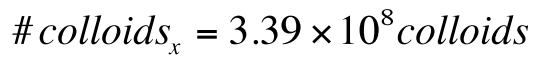

Visible Colloid per Liter in Detection Region:

Colloid $_{\text {visible }}=\frac{\text { \#colloids }_{x}}{\text { AdjustedVolume }_{x}}$

Colloid $_{\text {visible }}=\frac{3.39 \times 10^{8} \text { colloids }}{8.48 \times 10^{-11} \mathrm{~m}^{3}}$

Colloid $_{\text {visible }}=4.00 \times 10^{18}$ colloid $/ \mathrm{m}^{3}=4.00 \times 10^{15}$ colloid $/$ liter 
Number of visible colloids in the Detection Region:

\#VisibleColloids $=$ Colloid $_{\text {visible }} \times$ volume $_{x}$

\#VisibleColloids $=\left(4.00 \times 10^{15}\right.$ colloid $\left./ L\right)(0.986 \mu L)$

\#VisibleColloids $=3.95 \times 10^{9}$ colloids

It was previously found that the volume fraction from the detection region to the end of the lateral flow assay was 0.328 . The number of colloidal gold particles that must be in the initial sample was calculated.

Colloidal gold particles in initial sample:

$\#$ ColloidalGoldParticles $=\frac{(\# \text { VisibleColloids })}{(\text { VolumeFractionDetection })}$

\#ColloidalGoldParticles $=\frac{\left(3.95 \times 10^{9} \text { colloids }\right)}{0.328}$

\# ColloidalGoldParticles $=1.20 \times 10^{10}$ particles

Translated to moles:

VisibleMoles $_{\text {gold }}=\frac{\# \text { ColloidalGoldParticles }}{\text { Avogadro' } s \text { Number }}$

VisibleMoles $_{\text {gold }}=\frac{1.20 \times 10^{10} \text { particles }}{6.022 \times 10^{23} \text { molecules }}$

VisibleMoles $_{\text {gold }}=2.00 \times 10^{-14}$ moles

It was given that the molecular weight of the gold conjugated antibody was 45000

Daltons (or grams/mole). From this the mass was determined.

Mass of the Gold Conjugated Antibodies: 


$$
\begin{aligned}
& \text { mass }(\text { Au }- \text { Antibodies })=\left(M W_{\text {antibody }}\right)(\text { VisibleMoles }) \\
& \text { mass }(\text { Au }- \text { Antibodies })=(45000 \mathrm{~g} / \mathrm{mol})\left(2.00 \times 10^{-13} \text { moles }\right) \\
& \text { mass }(\text { Au }- \text { Antibodies })=9.00 \times 10^{-10} \mathrm{~g}
\end{aligned}
$$

Minimum concentration of Au-Antibody for detection:

$$
\begin{aligned}
& \text { MinimumGoldConcentration }_{\text {det ection }}=\frac{\operatorname{mass}(\text { Au }- \text { Antibodies })}{X \text { Volume }_{\text {reagent }}} \\
& \text { MinimumGoldConcentration }_{\text {det ection }}=\frac{9.00 \times 10^{-10} \mathrm{~g}}{0.986 \mu \mathrm{L}} \\
& \text { MinimumGoldConcentration }_{\operatorname{det} e c t i o n}=9.12 \times 10^{-4} \mathrm{mg} / \mathrm{ml}=0.912 \mu \mathrm{g} / \mathrm{ml}
\end{aligned}
$$

The molecular weight of the polyclonal capture antibody was given as $38200 \mathrm{~g} / \mathrm{mole}$.

Minimum concentration of Polyclonal Antibody for detection:

$$
\begin{aligned}
& \text { VisibleMoles }_{\text {polyclonal }}=\frac{\text { \#VisibleColloids }}{\text { Avogadro'sNumber }} \\
& \text { VisibleMoles }_{\text {polyclonal }}=\frac{3.95 \times 10^{9} \text { particles }}{6.022 \times 10^{23} \text { molecules }} \\
& \text { VisibleMoles }_{\text {polyclonal }}=6.56 \times 10^{-15} \text { moles } \\
& \text { mass }(\text { polyclonal })=\left(M_{\text {antibody }}\right)(\text { VisibleMoles }) \\
& \text { mass }(\text { polyclonal })=(38200 \mathrm{~g} / \mathrm{mol})\left(6.56 \times 10^{-15} \text { moles }\right) \\
& \operatorname{mass}(\text { polyclonal })=2.51 \times 10^{-10} \mathrm{~g} \\
& \text { MinimumGoldConcentration }_{\text {det ection }}=\frac{\text { mass }(\text { polyclonal })_{X \text { Volume }_{\text {reagent }}}}{\text { Molu }} \\
& \text { MinimumGoldConcentration }_{\text {det ection }}=\frac{2.51 \times 10^{-10} \mathrm{~g}}{0.986 \mu \mathrm{L}} \\
& \text { MinimumGoldConcentration }_{\operatorname{det} e c t i o n}=0.254 \mu \mathrm{g} / \mathrm{ml}
\end{aligned}
$$


Next the concentrations and volumes of VEGF must be found. From the AutoCAD chip design, the input can hold around $120 \mu \mathrm{L}$ of sample. Therefore, that will be the volume of VEGF that will be used.

For the concentration, it must be noted that every antibody holds two proteins. Therefore, the number of molecules of VEGF must be twice as much as the polyclonal antibody. Therefore:

\# moleculesVEGF $=(\#$ ColloidalGoldParticles $)(2)$

\# moleculesVEGF $=\left(1.20 \times 10^{10}\right.$ particles $)(2)$

\# moleculesVEGF $=2.40 \times 10^{10}$ particles

Next, using Avogadro's number, the number of moles may be calculated.

$$
\begin{aligned}
\text { molesVEGF } & =\frac{\# \text { moleculesVEGF }}{\text { Avogadros Number }} \\
\text { molesVEGF } & =\frac{2.40 \times 10^{10} \text { particles }}{6.022 \times 10^{23}} \\
\text { molesVEGF } & =4.00 \times 10^{-14} \text { moles }
\end{aligned}
$$

The given molecular weight of VEGF is $19200 \mathrm{~g} / \mathrm{mole}$. The mass and concentration of VEGF may be found.

$$
\begin{aligned}
& \text { massVEGF }=\text { moles } V E G F \times M W V E G F \\
& \text { massVEGF }=\left(4.00 \times 10^{-14} \text { moles }\right)(19200 \mathrm{~g} / \text { mole }) \\
& \text { massVEGF }=7.68 \times 10^{-4} \mu \mathrm{g}
\end{aligned}
$$




$$
\begin{aligned}
\text { concentrationVEGF } & =\frac{\text { mass VEGF }}{\text { volumeVEGF }} \\
\text { concentrationVEGF } & =\frac{7.68 \times 10^{-4} \mu g}{120 \mu L} \\
\text { concentrationVEGF } & =6.40 \times 10^{-6} \mathrm{mg} / \mathrm{ml}
\end{aligned}
$$




\section{Appendix H: SEM Images of Nitrocellulose}

Extra images were taken on the SEM of the HF 135 nitrocellulose with and without wax. The initial bare nitrocellulose membrane was taken with a 2500X objective (Figure 55). Then the impregnated wax membrane (Figure 56) and bare membranes were imaged at a 1000X objective (Figure 57).

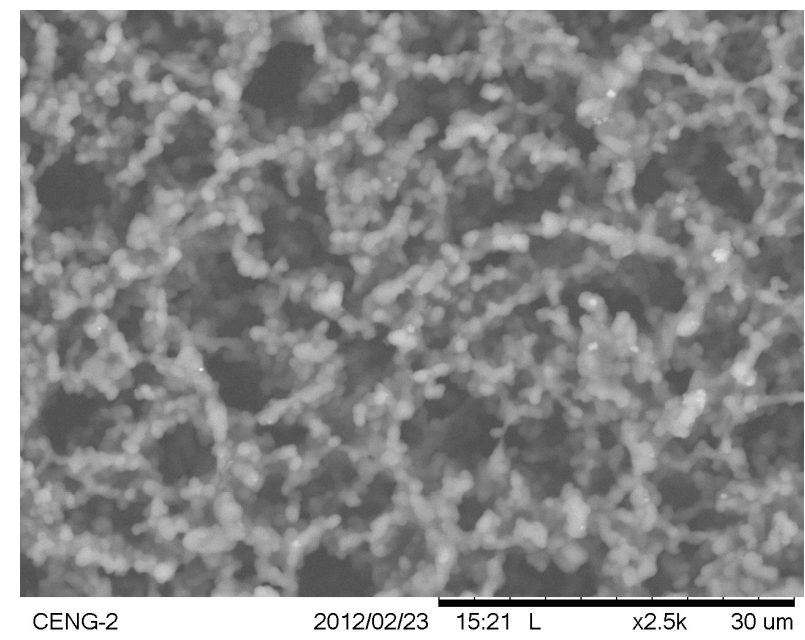

Figure 55: HF 135 SEM 2500X Objective. HF 135 backed nitrocellulose membrane without wax at $2500 \mathrm{X}$ lens.

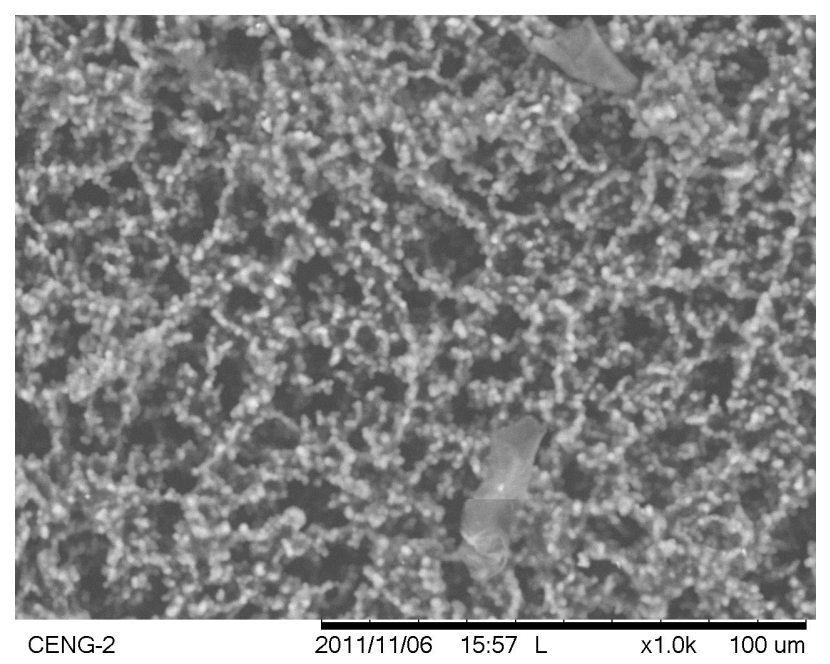

Figure 56: Wax HF 135 SEM 1000X Objective. HF 135 backed nitrocellulose membrane impregnated with wax in at $1000 \mathrm{X}$ magnification. 


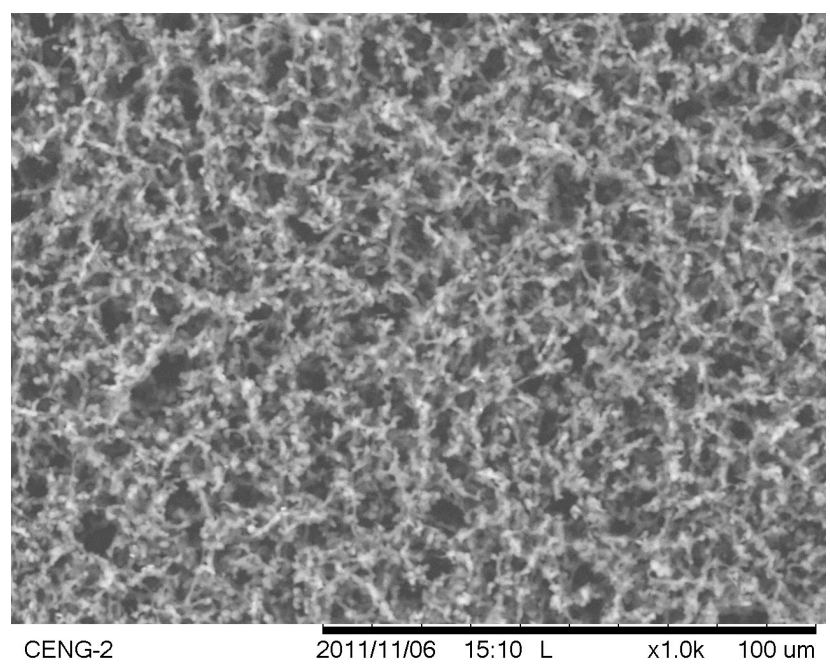

Figure 57: Bare HF 135 SEM 1000X Objective. HF 135 backed bare nitrocellulose membrane in at $1000 \mathrm{X}$ magnification. 